\title{
EXTRAÇÃO DE FATORES DE INTENSIDADE DE TENSÃO UTILIZANDO A SOLUÇÃO DO MÉTODO DOS ELEMENTOS FINITOS GENERALIZADOS
}

\begin{abstract}
Dissertação apresentada à Escola de Engenharia de São Carlos da Universidade de São Paulo, como parte dos requisitos para a obtenção do Título de Mestre em Engenharia de Estruturas.
\end{abstract}

Orientador: C. Armando Magalhães Duarte 
Ficha catalográfica preparada pela Seção de Tratamento da Informação do Serviço de Biblioteca - EESC/USP

Pereira, Jerônymo Peixoto Athayde
P436e Extração de fatores de intensidade de tensão utilizando a solução do método dos elementos finitos generalizados / Jerônymo Peixoto Athayde Pereira. -São Carlos, 2004 .

Dissertação (Mestrado) - Escola de Engenharia de São Carlos-Universidade de São Paulo, 2004.

Área: Engenharia de Estruturas.

Orientador: Prof. C. Armando Magalhães Duarte.

1. Métodos de extração. 2. Fatores de intensidade de tensão. 3. MEFG. 4. Método da integral de contorno. 5. Método da função cutoff. 6. Integral-J. I. Título. 
FOLHA DE JULGAMENTO

Candidato: Engenheiro JERÔNYMO PEIXOTO ATHAYDE PEREIRA

Dissertaçào delendida e julgada em 04-05-2004 perante a Connissáo Julgadora:

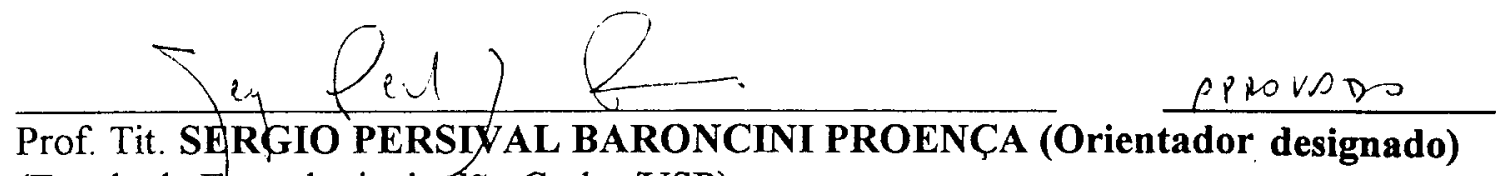
(Escola de Engenharia de São Carlos/USP)

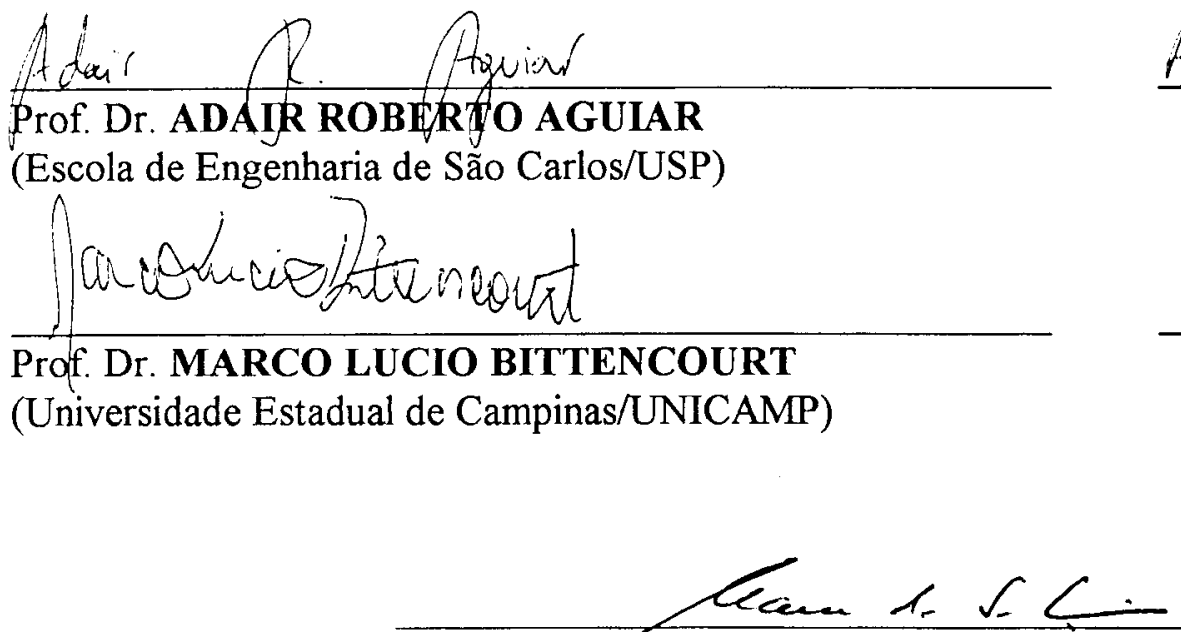

AprovaDo

Aprovado

Prof Assoc. MARCIO ROBERTO SILVA CORRÊA

Coordenador do Programa de Pós-Graduação em Engenharia Civil (Engenharia de Estruturas)

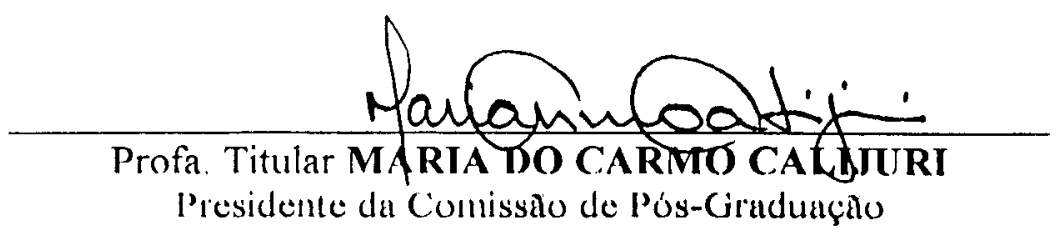


Aos meus pais, aos meus irmãos e à Caroline, com amor. 


\section{AGRADECIMENTOS}

A Deus pela saúde e disposição para transpor mais essa etapa.

Aos meus pais e irmãos pelo apoio e incentivo na realização dos meus ideais.

À Caroline pelo carinho, compreensão e por estar ao meu lado em todos os momentos.

Ao professor Carlos Armando Magalhães Duarte pela orientação e ensinamentos ministrados, sobretudo, pela paciência e assistência demonstrada durante $o$ desenvolvimento deste trabalho.

Aos professores do Centro de Tecnologia da UFAL, em especial aos professores Roberaldo Carvalho e João Barbirato, pelo estímulo e apoio amigo sempre dispensado.

Aos amigos Cilmar Basaglia, Claudius Barbosa, Edson Costa, Geilson Vasconcelos, Larissa Kirchhof, Márcio Félix, Tiago Thomazelli e Wilson Wutzow pelo companheirismo e pelos momentos de alegria em todas as atividades desenvolvidas ao longo do curso.

Aos colegas de república Valber Pedrosa e Walter Oliveira pelo tempo de aprendizado e vivência dividindo a mesma morada.

Agradeço aos professores e funcionários do Departamento de Estruturas (SET/EESC) que, de alguma forma, contribuíram para o desenvolvimento deste trabalho.

À CAPES (Coordenação de Aperfeiçoamento de Pessoal de Nível Superior) pelo suporte financeiro desta pesquisa. 


\section{RESUMO}

PEREIRA, J. P. A. (2004). Extração de fatores de intensidade de tensão utilizando a solução do método dos elementos finitos generalizados. Dissertação (Mestrado) Escola de Engenharia de São Carlos, Universidade de São Paulo, São Carlos, 2004.

O trabalho apresenta uma análise do desempenho de vários métodos de extração de fatores de intensidade de tensão a partir de soluções numéricas obtidas com o método dos elementos finitos generalizados (MEFG). A convergência dos fatores de intensidade de tensão é comparada com a da energia de deformação a fim de investigar a superconvergência dos métodos. Para extração dos fatores de intensidade de tensão e o cálculo da taxa de energia disponibilizada para propagação da fissura, implementam-se os métodos da integral de contorno (MIC), da função cutoff (MFC) e da integral-J no contexto do MEFG. Desenvolve-se a formulação dos métodos de extração de forma a obter uma implementação independente da malha utilizada na modelagem do problema. Aplica-se a extração dos fatores de intensidade de tensão, para modos puros e mistos, em problemas clássicos da mecânica da fratura. Verifica-se a convergência dos fatores de intensidade de tensão e da taxa de energia disponibilizada para a propagação da fissura, obtidos com cada método de extração, com o enriquecimento da ordem polinomial da solução do MEFG. Investiga-se a robustez dos métodos com relação ao tamanho dos domínios de extração.

Palavras-chave: métodos de extração, fatores de intensidade de tensão, MEFG, método da integral de contorno, método da função cutoff, integral-J 


\begin{abstract}
PEREIRA, J. P. A. (2004). Extraction of stress intensity factors from generalized finite element solutions. M.Sc. Dissertation - Escola de Engenharia de São Carlos, Universidade de São Paulo, São Carlos, 2004.
\end{abstract}

The performance of several techniques to extract stress intensity factors (SIF) from numerical solutions computed with the generalized finite element method (GFEM) is investigated. The convergence of the stress intensity factors is compared with the convergence of strain energy with the aim of investigate the superconvergence of the methods. The contour integral (CIM), the cutoff function (CFM) and the J-integral methods are considered to compute stress intensity factors and energy release rate. The proposed implementation of the extraction techniques is completely independent of the discretization used. Several numerical examples demonstrating the convergence of the computed stress intensity factors and the energy release rate, with the increasing of $p$ order of the GFEM solution, are presented.

Keywords: extraction methods, stress intensity factors, GFEM, contour integral method, cutoff function method, J-integral. 


\section{SUMÁRIO}

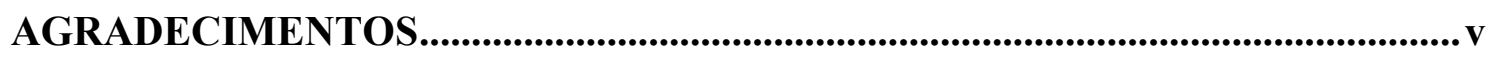

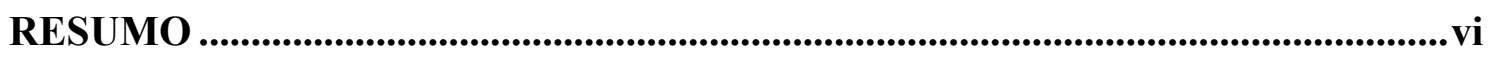

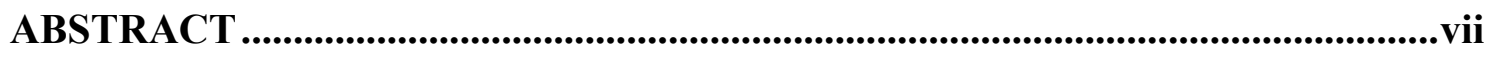

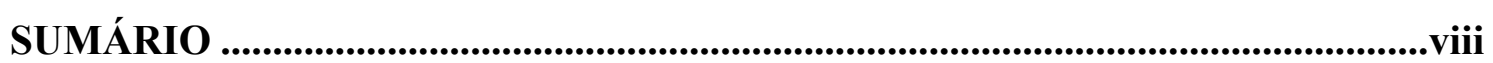

1 INTRODUÇÃ

1.1 MECÂNICA DA FRATURA - PARÂMETROS E APLICAÇÕES .................................... 1

1.2 SOBRE O MÉTODO DOS ELEMENTOS FINITOS GENERALIZADOS (MEFG) ..............

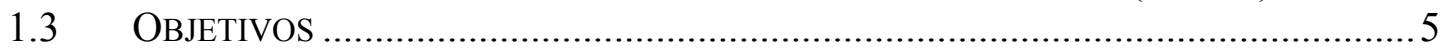

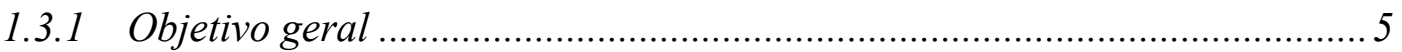

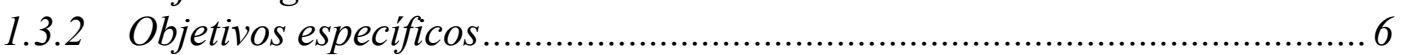

1.4 REVISÃO BIBLIOGRÁFICA........................................................................ 7

1.4.1 Métodos de extração baseados na Integral-J ............................................ 7

1.4.2 Métodos de extração superconvergentes ................................................. 14

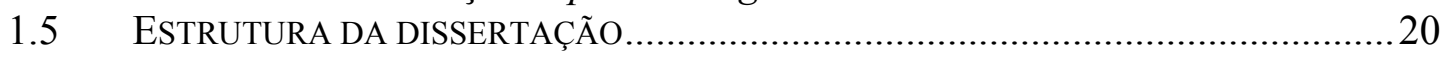

2 PROBLEMAS ELÁSTICOS COM PONTOS SINGULARES .........................21

2.1 CONCEITOS BÁSICOS DE MECÂNICA DA FratURA ….......................................21

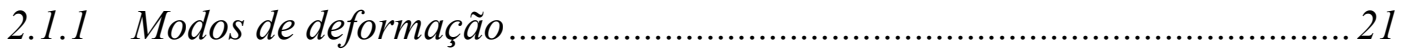

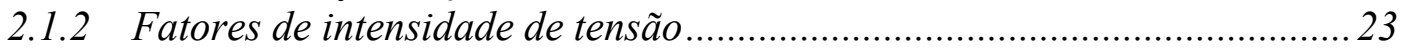

2.2 DESCRIÇÃO DA SOLUÇÃO EXATA EM UM DOMÍNIO COM PONTOS SINGULARES.. 24

2.3 SOLUÇÃO EXATA BIDIMENSIONAL NA VIZINHANÇA DE PONTOS SINGULARES ... 25

3 FORMULAÇÃO DOS MÉTODOS DE EXTRAÇÃO ..................................33

3.1 INTEGRAL INDEPENDENTE DO CAMINHO - LEI DE BETTI ....................................33

3.2 ORTOGONALIDADE DAS AUTOFUNÇÕES …..................................................... 38

3.3 EXTRAÇÃO DOS FATORES DE INTENSIDADE DE TENSÃO..................................4 41

3.3.1 Método da Integral de Contorno (MIC) ..................................................... 42

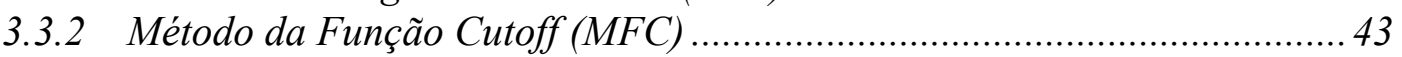

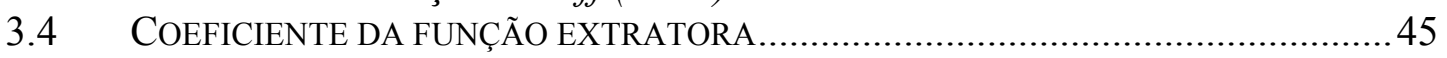

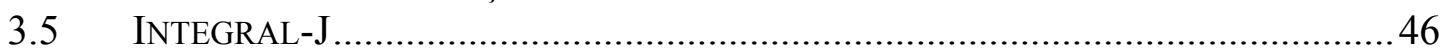

4 ASPECTOS DA FORMULAÇÃO NUMÉRICA DOS MÉTODOS DE

EXTRAÇÃO .............................................................................................48

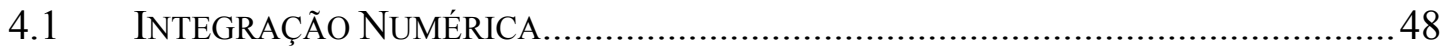

4.1.1 Quadratura Gaussiana (Gauss-Legendre) .................................................. 49

4.2 FORMULAÇÃO NUMÉRICA DOS MÉTODOS DE EXTRAÇÃO .................................51

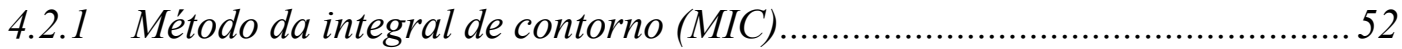

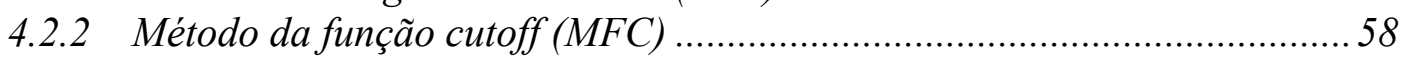

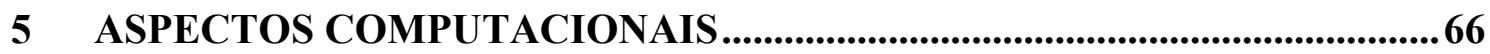

5.1 O PARAdigma da ProgramaÇão OriEntada A OBJETOS (POO) ...................66

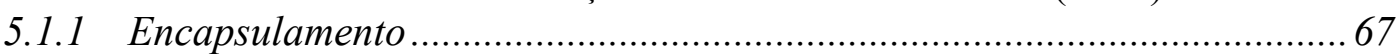

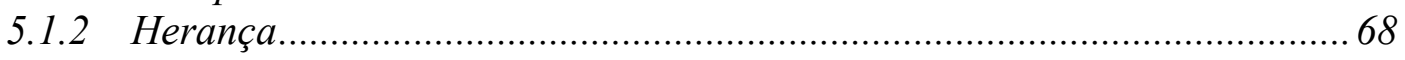




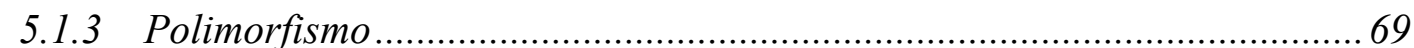

5.2 O PROGRAMA SET (SCIENTIFIC AND ENGINEERING TOOLKIT) ..........................69

5.2.1 Classe geometria do elemento (GeoEl) ...................................................... 70

5.2.2 Classe elemento computacional (CompEl) ................................................ 71

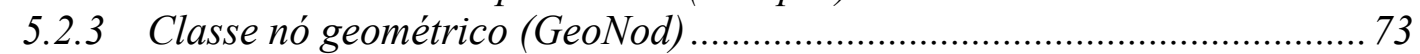

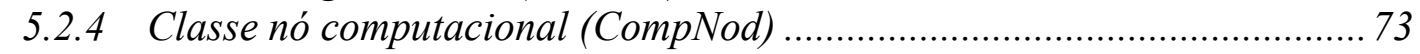

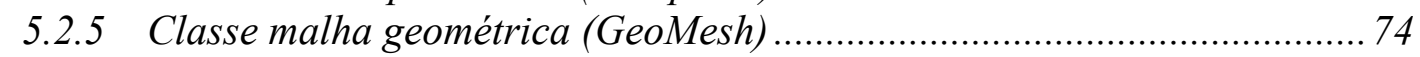

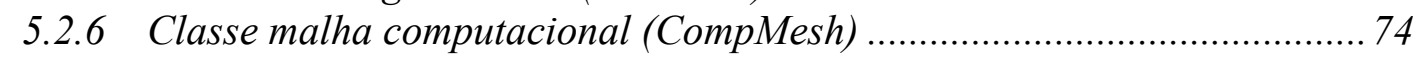

5.2.7 Classe material ou equação diferencial (Material) .................................... 75

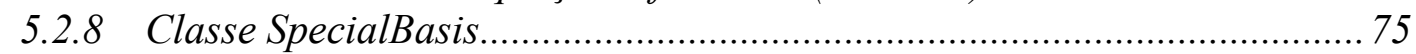

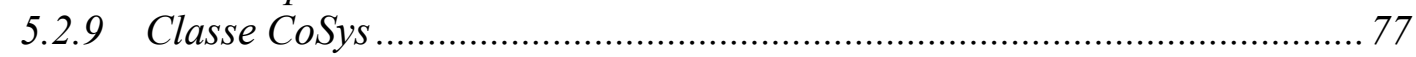

5.2.10 Classes para integração numérica (IntPoints e IntRuleList).................. 77

5.2.11 Classe para etapa de pré-processamento (DataFileI)........................... 79

5.2.12 Classe para etapa de análise (Analysis) ................................................ 80

5.2.13 Classes para representação gráfica (GraphEl e GraphMesh)...............81

5.2.14 Classe para etapa de pós-processamento (DataFileO) ...........................82

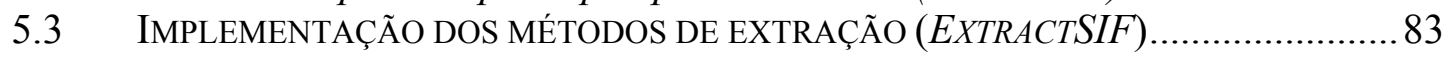

5.3.1 Classe para o método de extração MIC (ExtractCIM2D) ........................... 84

5.3.2 Classe para o método de extração MFC (ExtractCFM2D) .........................85

5.3.3 Classe para o método da Integral-J (ExtractJ2D).................................. 87

6 RESULTADOS NUMÉRICOS.................................................................89

6.1 PAINEL FRATURADO COM CONDIÇÕES DE CONTORNO ESPECIAIS ......................89

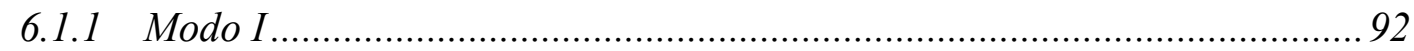

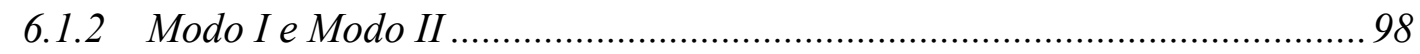

6.2 PAINEL FRATURADO - TENSÃO CONSTANTE E FISSURA HORIZONTAL ............... 105

6.3 PAINEL FRATURADO - FISSURA INCLINADA ................................................ 110

6.4 FISSURA HORIZONTAL CENTRADA INSERIDA NO DOMÍNIO ............................. 115

6.5 FISSURA INCLINADA CENTRADA INSERIDA NO DOMÍNIO (COM VARIAÇÃO DO

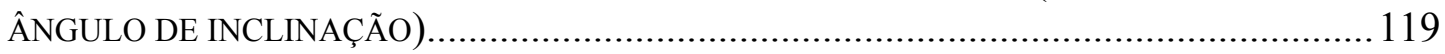

6.6 FISSURA INCLINADA EXCÊNTRICA INSERIDA NO DOMÍNIO .............................. 126

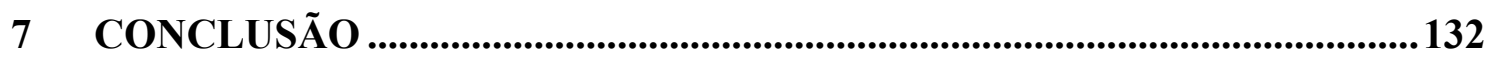

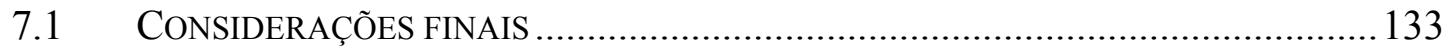

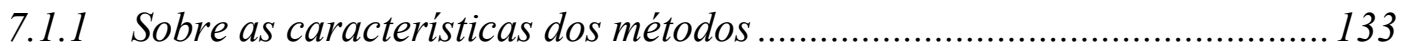

7.1.2 Sobre a formulação numérica ............................................................... 134

7.1.3 Sobre a implementação computacional .................................................. 134

7.2 PROPOSTAS PARA TRABALHOS FUTUROS .................................................. 135

REFERÊNCIAS BIBLIOGRÁFICAS ................................................................136

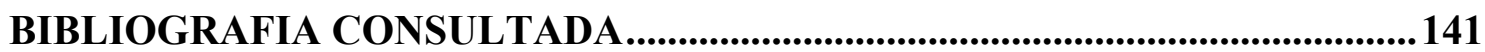

APÊNDICE A - TRANSFORMAÇÃO DE COORDENADAS PARA O MÉTODO

DA INTEGRAL-J .....................................................................................................142

APÊNDICE B - DESENVOLVIMENTO ANALÍTICO PARA OS

COEFICIENTES DA FUNÇÃO EXTRATORA ................................................ 146

APÊNDICE C - TRANSFORMAÇÃO DE COORDENADAS PARA AS DERIVADAS DA FUNÇÃO EXTRATORA DO MÉTODO DA FUNÇÃO 


\section{Introdução}

\subsection{Mecânica da fratura - parâmetros e aplicações}

A ocorrência de falhas estruturais por propagação de fissuras está associada a problemas de análise e projeto em diversos campos da engenharia. Nesse sentido, o desenvolvimento de métodos para quantificar os efeitos da presença de fissuras em elementos estruturais tem conduzido à evolução da teoria da mecânica da fratura.

As formas de avaliar a estabilidade de determinada fissura e sua maneira de propagação são aplicações importantes da mecânica da fratura. A análise do comportamento de uma fissura, em um material submetido a diversas condições de carregamento, envolve a avaliação de parâmetros tais como a taxa de energia disponibilizada pelo sistema para a propagação da fissura $(\mathscr{G})$ e os fatores de intensidade de tensão $(\mathrm{K})$, cujos valores estão diretamente relacionados.

Com a determinação dos valores de $\mathrm{K}$ referentes a cada modo de deformação (Figuras 2.1, 2.2 e $2.3-\mathrm{K}_{\mathrm{I}}, \mathrm{K}_{\mathrm{II}}$ e $\mathrm{K}_{\mathrm{III}}$ ), em um material elástico linear, definem-se os campos que regem o comportamento das tensões e deslocamentos na região próxima à ponta (aresta no caso tri-dimensional) de uma fissura. Os valores $\mathrm{K}_{\mathrm{I}}, \mathrm{K}_{\mathrm{II}}$ e $\mathrm{K}_{\mathrm{III}}$ também são utilizados na determinação da direção e da velocidade com que a fissura se propaga.

A propagação de uma fissura ocorre quando $\mathscr{G}$ excede um determinado valor crítico. O valor $\mathscr{G}$ crítico pode ser admitido como um parâmetro do material. Com base nesse valor crítico, determina-se a intensidade da fratura para fissuras de qualquer tamanho em um mesmo material. É possível avaliar também o tamanho máximo de fissura que o material suporta sob determinado estado de tensão. Portanto, o valor $\mathscr{G}$ crítico é uma medida da resistência do material à fissuração. 
O balanço de energia serve como base na formulação de um critério para a propagação de fissura. Segundo Griffith, a condição necessária para a propagação de uma fissura é que o sistema possa disponibilizar a quantidade de energia a ser despendida para o aumento de sua superfície atual (BROEK, 1982). Esse critério energético aplica-se, de uma forma geral, a materiais em que os defeitos iniciais são considerados desprezíveis e a resposta do material é elástica linear.

Utilizando o critério energético de Griffith, Irwin apresentou uma relação direta entre os fatores de intensidade de tensão, referentes a cada modo de deformação, e a taxa de energia disponibilizada para a propagação da fissura em materiais elásticos lineares (OWEN; FAWKES, 1983).

Para problemas em que as deformações plásticas não são desprezadas, a integralJ, desenvolvida por Rice (1968), fornece o valor da taxa de energia disponibilizada para a propagação da fissura. Rice (1968) mostrou que a integral-J, quando definida ao longo de um contorno em volta da ponta da fissura, é a mudança na energia potencial total com relação a extensão virtual da fissura. Aplicando-se esse resultado a materiais com regime elástico linear, encontra-se o mesmo valor determinado por Griffith para a taxa de energia disponibilizada para a propagação da fissura. Portanto, a integral-J pode ser um critério de fratura aplicável a problemas elásticos lineares bem como para fraturas plásticas.

Como conseqüência da presença de fisssuras, o valor de esforço limite para o qual uma estrutura foi projetada é reduzido. A resistência residual da estrutura decresce progressivamente com o aumento do tamanho da fissura. Sendo assim, algumas estruturas são projetadas para suportar cargas de serviço altas o suficiente para dar início à fissuração, particularmente quando defeitos ou concentrações de tensão estão presentes (OWEN; FAWKES, 1983).

Em virtude da disponibilidade de combinações de materiais e métodos de análise mais detalhados, o projetista tem a oportunidade de aperfeiçoar seus projetos para obter produtos mais seguros, esbeltos e com custo reduzido. Cabe ao projetista antecipar a possibilidade de fissuração da estrutura e conseqüentemente admitir a possibilidade de falha estrutural.

Em determinadas análises é importante para o projetista poder simular o caminho com que a fissura se propaga. O exemplo descrito a seguir ilustra a necessidade de investigar a direção de propagação de uma fissura. Trata-se da análise do 
comportamento de uma fissura em uma engrenagem utilizada em motores de helicópteros.
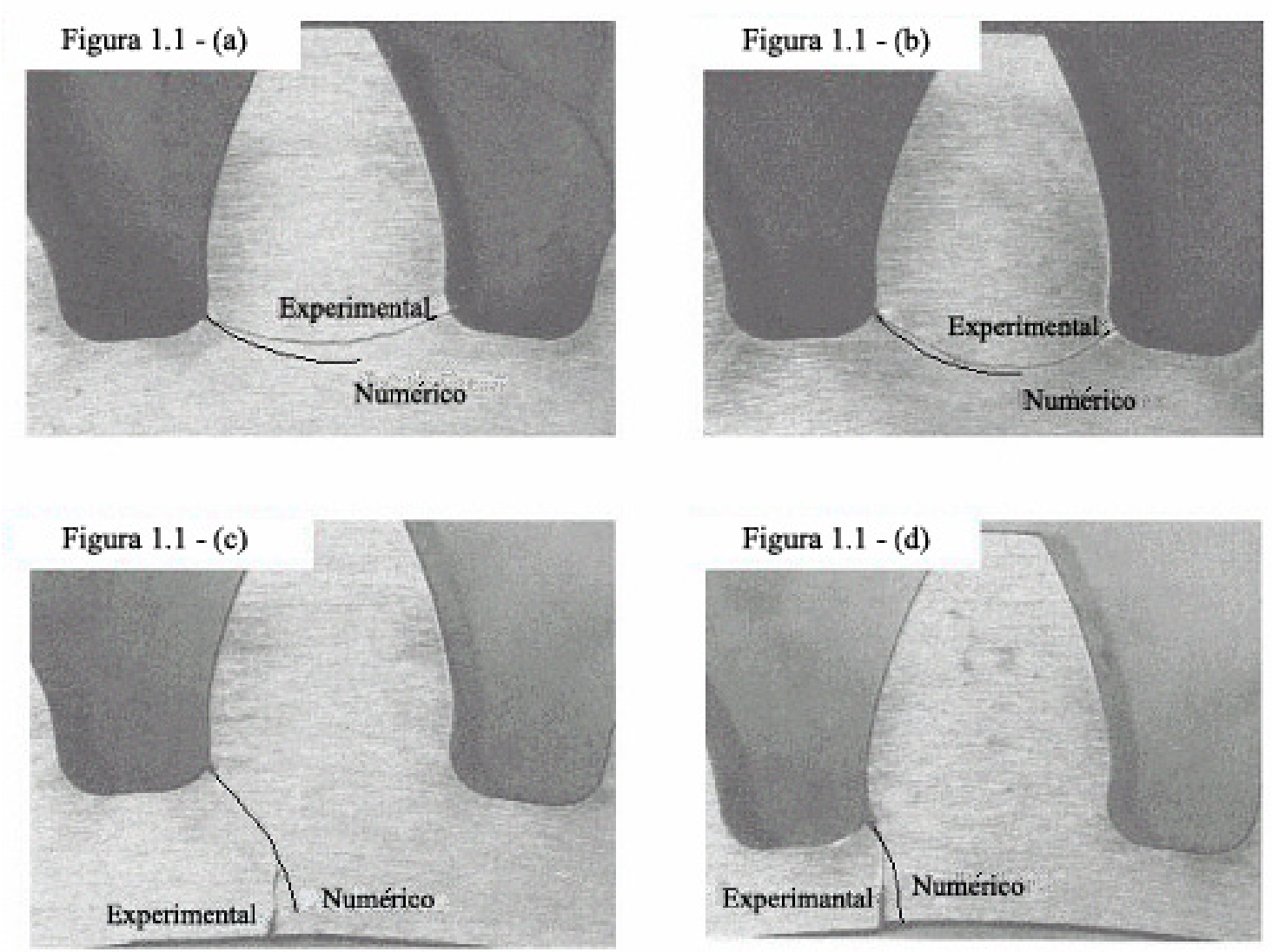

Figura 1.1 - Análise da direção de propagação de uma fissura. (a) e (b) rompimento de um dente da engrenagem (c) e (d) colapso da engrenagem. Adaptada de Cornell (2003).

Dependendo da direção de propagação da fissura, pode ocorrer o rompimento de apenas um dente da engrenagem (Figura 1.1- (a) e (b)) ou o rompimento da engrenagem como um todo (Figura 1.1- (c) e (d)). No primeiro caso (rompimento de apenas um dente da engrenagem), o piloto pode fazer um pouso de emergência e evitar um acidente grave. No segundo caso (colapso total da engrenagem), o motor pára de girar podendo gerar conseqüências catastróficas.

Esse exemplo ilustra a necessidade de se obter valores para $\mathrm{K}_{\mathrm{I}}, \mathrm{K}_{\mathrm{II}}$ e $\mathrm{K}_{\mathrm{III}}$ de forma precisa e confiável e não apenas o valor de $\mathscr{G}$ ou J. A aplicação de métodos de extração dos fatores de intensidade de tensão, comumente encontrados na literatura, torna possível a análise desse tipo de problema. Uma breve revisão bibliográfica dos principais métodos de extração é apresentada na seção 1.3. 


\subsection{Sobre o método dos elementos finitos generalizados (MEFG)}

O método dos elementos finitos generalizados (MEFG) pode ser interpretado como uma forma distinta de geração das funções de forma de elementos finitos (Figura 1.2). As funções de forma no MEFG podem ser constituídas utilizando-se funções polinomiais, como no MEF, ou qualquer outra classe de funções que tenha boas propriedades aproximadoras (DUARTE; BABUŠKA; ODEN, 1999). O MEFG foi proposto, independentemente, por Babuška e colegas utilizando os nomes: Método da partição da unidade (BABUŠKA; MELENK, 1997), e MEFG (STROBOULIS; COPPS; BABUŠKA, 2000) e por Duarte e Oden com os nomes: Método das nuvens hp (DUARTE, 1996) e MEF baseado no método das nuvens hp (ODEN; DUARTE e ZIENKIEWICZ, 1998).

Z

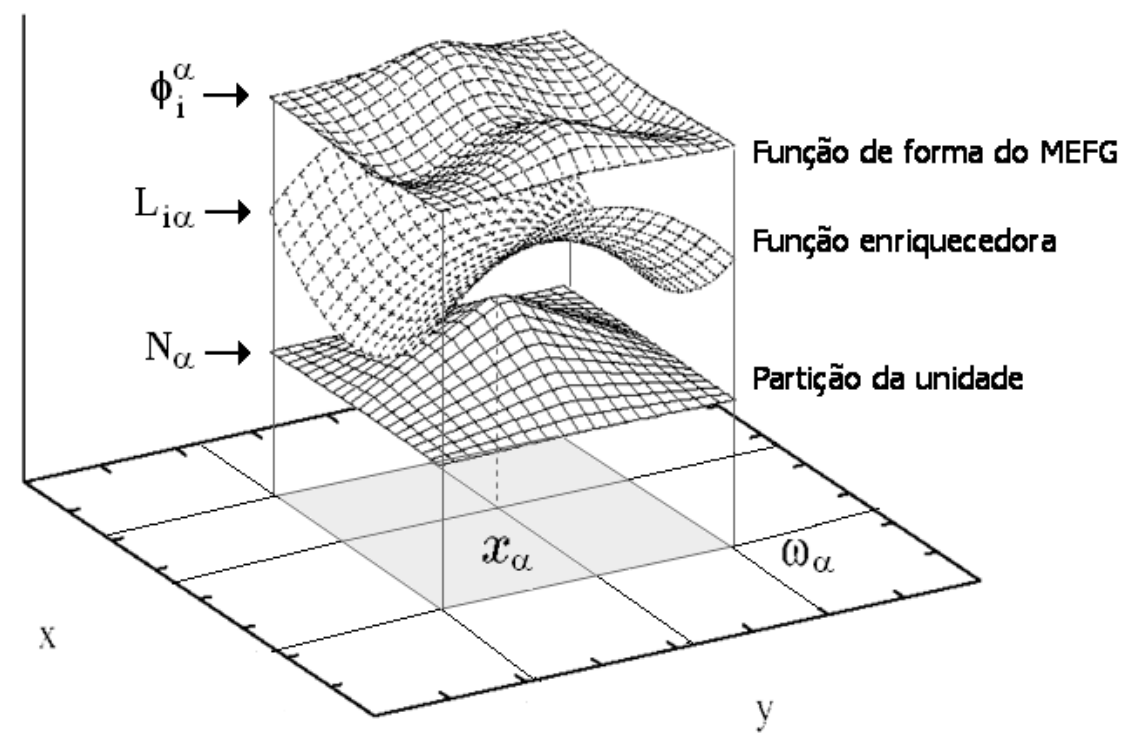

Figura 1.2 - Função de forma do MEFG. Adaptada de Barros (2002).

A construção das funções de forma do MEFG (Figura 1.2) pode ser descrita matematicamente da seguinte forma:

Considere, por exemplo, uma malha de elementos finitos convencional formada por quadriláteros mostrada na Figura 1.2, na qual funções de forma globais $\mathrm{N}_{\alpha}$ são construídas em cada ponto nodal $x_{\alpha}, j=1,2, \ldots, n$. Estas funções são tais que: 


$$
\sum_{\alpha=1}^{\mathrm{n}} \mathrm{N}_{\alpha}(\mathrm{x})=1 \quad \text { em qualquer } \mathrm{x} \in \bar{\Omega}
$$

ou seja, as funções de forma $\mathrm{N}_{\alpha}$ formam uma partição da unidade (PU) (ODEN; REDDY, 1976). Em (1.1), $\bar{\Omega}$ é o domínio de análise e o seu contorno.

Seja $\omega_{\alpha}$ o conjunto formado pelos elementos que compartilham o nó $\mathrm{x}_{\alpha}$, conforme ilustrado na Figura 1.2. Seja $\mathrm{L}_{\alpha}=\left\{1, \mathrm{~L}_{\alpha 1}, \mathrm{~L}_{\alpha 2}, \ldots, \mathrm{L}_{\alpha \mathrm{n}}\right\}$ uma base geradora para um espaço de funções $\chi_{\alpha}\left(\omega_{\alpha}\right)$ definido sobre $\omega_{\alpha}$. As funções de forma para o método dos elementos finitos generalizados, associadas ao nó $\mathrm{x}_{\alpha}$, são então definidas por:

$$
\left\{\phi_{\mathrm{i}}^{\alpha}\right\}_{\mathrm{i}=1}^{\mathrm{n}}=\mathrm{N}_{\alpha} \cdot\left\{1, \mathrm{~L}_{\alpha 1}, \mathrm{~L}_{\alpha 2}, \ldots, \mathrm{L}_{\alpha \mathrm{n}}\right\}
$$

A escolha mais evidente para as funções de enriquecimento $L_{\alpha}$ (base do espaço local $\left.\chi_{\alpha}\left(\omega_{\alpha}\right)\right)$ são as funções polinomiais, as quais podem aproximar funções suaves de forma satisfatória. Neste caso, o MEFG é essencialmente idêntico ao método dos elementos finitos convencional. Maiores detalhes sobre o MEFG podem ser encontrados nas referências (ODEN; DUARTE; ZIENKIEWICZ, 1998; DUARTE; BABUŠKA; ODEN, 1999; STROBOULIS; COPPS; BABUŠKA, 2000; BARROS, 2002).

\subsection{Objetivos}

\subsubsection{Objetivo geral}

Como objetivo geral para o presente trabalho, propõe-se:

- Aprofundar os conhecimentos nos fundamentos da mecânica da fratura computacional e no método dos elementos finitos e suas generalizações, particularmente o método dos elementos finitos generalizados (MEFG), por meio de uma implementação para extração de fatores de intensidade de tensão, 
Implementam-se os métodos de extração de fatores de intensidade de tensão em um código computacional desenvolvido pelo orientador deste trabalho. Tal programa implementa o MEFG utilizando os recursos de programação orientada a objetos. $\mathrm{O}$ estudo do MEFG, no presente trabalho, é feito tanto em nível teórico, como também em relação a aspectos de implementação. Contudo, em virtude da extensão do assunto e do enfoque do trabalho ser voltado para extração dos fatores de intensidade de tensão a apresentação teórica do MEFG limita-se a Seção 1.2. Maiores detalhes podem ser encontrados nas referências citadas naquela seção.

\subsubsection{Objetivos específicos}

No presente trabalho, propõe-se:

- Revisão e estudo dos métodos de extração de fatores de intensidade de tensão disponíveis para o método dos elementos finitos. Esse estudo concentra-se no método da integral de contorno (MIC) (SZABÓ; BABUŠKA, 1988) e no método da função cutoff (MFC) (SZABÓ; BABUŠKA, 1988). Justifica-se tal escolha pela generalidade, fundamentação matemática, robustez demonstrada no contexto do MEF e eficiência computacional dos referidos métodos. Adicionalmente, a formulação do MIC e do MFC é geral suficiente para que esses métodos possam ser utilizados, por exemplo, com o método dos elementos finitos generalizados;

- Implementação computacional do MIC e do MFC em um programa para a solução de problemas da elasticidade linear baseado no método dos elementos finitos generalizados. A aplicação dos métodos de extração concentra-se na análise de problemas elásticos bidimensionais. Não houve a necessidade de nenhuma grande modificação na formulação do MIC e do MFC. As modificações aplicadas aos métodos estão relacionadas com aspectos de implementação dos mesmos. Esta implementação foi desenvolvida de modo que sua extensão para o caso tridimensional seja da forma mais simples possível. No cálculo das integrais de contorno e domínio, a malha de elementos finitos não é utilizada;

- Análise crítica do desempenho dos métodos de extração de fatores de intensidade de tensão implementados. Analisa-se não só a performance relativa 
de cada método, mas também a robustez dos mesmos em relação ao refinamento e/ou enriquecimento polinomial do espaço solução.

\subsection{Revisão Bibliográfica}

A aplicação dos princípios da mecânica da fratura é amplamente voltada para os fatores de intensidade de tensão. Nas últimas décadas, diversas pesquisas têm sido direcionadas para o desenvolvimento de métodos de extração dos fatores de intensidade de tensão.

Para problemas mais simples, é possível obter os valores dos fatores de intensidade de tensão analiticamente. Porém, para a maior parte dos problemas de interesse prático, o desenvolvimento analítico completo não é possível. Na análise desses problemas mais complexos, faz-se necessário o uso da solução de métodos numéricos para extração dos fatores de intensidade de tensão, particularmente o Método de Elementos Finitos (MEF) e suas generalizações, o Método dos Elementos de Contorno, etc.

No presente trabalho, os métodos de extração foram divididos em dois grupos, os quais serão descritos a seguir.

\subsubsection{Métodos de extração baseados na Integral-J}

Dentre os métodos de extração que utilizam a solução do MEF, destacam-se como mais utilizados os que possuem sua formulação baseada na integral-J.

Rice (1968) apresentou uma integral de linha que possui o mesmo valor para todos os caminhos que envolvem a região próxima à ponta da fissura. Considerando uma fissura em uma placa submetida a uma carga arbitrária, assumida remota em relação à fissura, com um caminho fechado arbitrário em torno da fissura (Figura 1.3). Assim, na ausência de forças de volume, a integral $\mathrm{J}_{\mathrm{x}_{\mathrm{k}}}$ é definida da seguinte forma: (RAJU; SHIVAKUMAR, 1990)

$$
\mathrm{J}_{\mathrm{x}_{\mathrm{k}}}=\int_{\Gamma}\left(\mathrm{Wn}_{\mathrm{k}}-\sigma_{\mathrm{ij}} \frac{\partial \mathrm{u}_{\mathrm{i}}}{\partial \mathrm{x}_{\mathrm{k}}} \mathrm{n}_{\mathrm{j}}\right) \mathrm{d} \Gamma
$$

onde $\mathrm{W}$ é a densidade de energia de deformação total. 


$$
\mathrm{W}=\int_{0}^{\varepsilon_{\mathrm{ij}}} \sigma_{\mathrm{ij}} \mathrm{d} \varepsilon_{\mathrm{ij}}
$$

Nas equações (1.3) e (1.4), $\sigma_{\mathrm{ij}}$ é o tensor de tensão, $\varepsilon_{\mathrm{ij}}$ é a deformação total (a qual inclui a soma das deformações elásticas e plásticas) e $n_{j}$ é a j-ésima componente do vetor $\mathbf{n}$ normal ao contorno $\Gamma$ (Figura 1.3). No caso bidimensional, os índices i, j e k recebem os valores 1 e 2. $\mathrm{x}_{1}$ e $\mathrm{x}_{2}$ representam as direções tangencial e normal à ponta da fissura, respectivamente (Figura 1.3).

Na equação (1.3) adotando $\mathrm{k}=1,2\left(\mathrm{~J}_{\mathrm{x}_{1}}\right.$ e $\left.\mathrm{J}_{\mathrm{x}_{2}}\right)$, têm-se as integrais de caminho independente que definem a quantidade total de fluxo de energia que deixa o contorno $\Gamma$ na direção $x_{1}$ e $x_{2}$, respectivamente. A integral $J_{x_{1}}$ é comumente chamada de integral-J (RICE, 1968; MORAN; SHIH, 1987 a, b; RAJU; SHIVAKUMAR, 1990) e a integral $\mathrm{J}_{\mathrm{x}_{2}}$ é denominada de integral do produto (RAJU; SHIVAKUMAR, 1990).

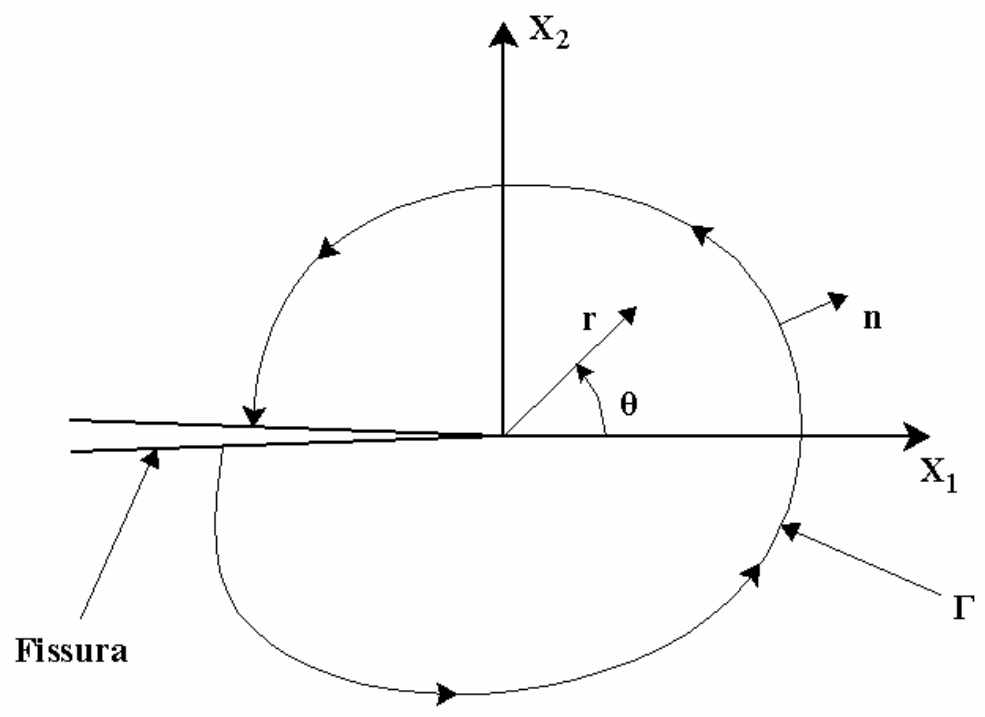

Figura 1.3 - Contorno típico para avaliação da integral - J.

A integral da equação (1.3) permite avaliar a variação do potencial de energia em relação ao avanço virtual da fissura em problemas bidimensionais. O valor J é a taxa de redução do potencial de energia com relação ao comprimento da fissura para materiais em que os efeitos de plasticidade não são desprezados. A integral-J fornece a generalização da conexão entre os fatores de intensidade de tensão e a taxa de energia 
disponibilizada para a propagação da fissura, inicialmente proposta por Irwin para materiais lineares. É possível demonstrar que a integral-J, quando aplicada a materiais elásticos lineares, é equivalente à formulação de Irwin (RICE, 1968).

A resposta numérica dos campos de deslocamento e de tensão e a avaliação da integral de linha na vizinhança da ponta da fissura são as principais fontes de resultados imprecisos e que dificultam a aplicação do método da integral-J.

\subsubsection{Método da derivada da rigidez}

Parks (1974) desenvolveu a técnica da derivada da rigidez para a determinação da taxa de energia disponibilizada para a propagação da fissura e dos fatores de intensidade de tensão, utilizando a solução do MEF. Esta técnica foi baseada na variação do potencial de energia com relação ao comprimento da fissura, o que implica na variação da rigidez do sistema com o avanço da fissura. Parks (1974) mostrou que a técnica da derivada da rigidez não requer o uso de elementos especiais na ponta da fissura, bem como uma segunda solução do MEF, para pequenas modificações no comprimento da fissura. Utilizando elementos triangulares com deformação constante, Parks (1974) mostrou que a técnica da derivada da rigidez é numericamente equivalente ao método da integral-J. O método desenvolvido por Parks (1974) pode ser interpretado como uma forma alternativa do método da extensão virtual da fissura.

\subsubsection{Método da extensão virtual da fissura}

Hellen (1975) utilizou a técnica de extensão virtual da fissura, juntamente com o MEF, para a determinação da taxa de energia disponibilizada para a propagação da fissura. Hellen (1975) mostrou que o uso da técnica de diferença de energia com pequenas mudanças no tamanho da fissura e a modelagem do problema com elementos finitos de alta ordem polinomial e elementos especiais na ponta da fissura levam a resultados precisos. No trabalho de Hellen (1975) utilizou-se o critério da taxa máxima de energia disponibilizada para determinar a direção de propagação da fissura. Com esta técnica é possível determinar o fator de intensidade de tensão para modos puros e a direção de propagação da fissura para modos mistos. A desvantagem desse método está relacionada com a impossibilidade de determinação dos valores de $K_{I}, K_{I I}$ e $K_{\text {III }}$ individualmente para modos mistos. 


\subsubsection{Técnica da integral de domínio equivalente}

Nesta técnica, a integral-J é transformada em uma integral de domínio equivalente (IDE) (LI, SHIH; NEEDLEMAN, 1985; MORAN; SHIH, 1987 a, b). Na transformação utilizam-se funções ponderadoras em uma região formada por um caminho fechado próximo a ponta da fissura (Figura 1.4). Utilizando a equação (1.3) em um caminho fechado (Figura 1.4), multiplicando o integrando por uma função ponderadora $\mathrm{S}$ e aplicando o teorema da divergência, tem-se:

$$
\left(\mathrm{J}_{\mathrm{x}_{\mathrm{k}}}\right)_{\text {domínio }}=-\int_{\mathrm{A}}\left(\frac{\partial(\mathrm{WS})}{\partial \mathrm{x}_{\mathrm{k}}}-\frac{\partial\left(\sigma_{\mathrm{ij}} \frac{\partial \mathrm{u}_{\mathrm{i}}}{\partial \mathrm{x}_{\mathrm{k}}} \mathrm{S}\right)}{\partial \mathrm{x}_{\mathrm{j}}}\right) \mathrm{dA}
$$

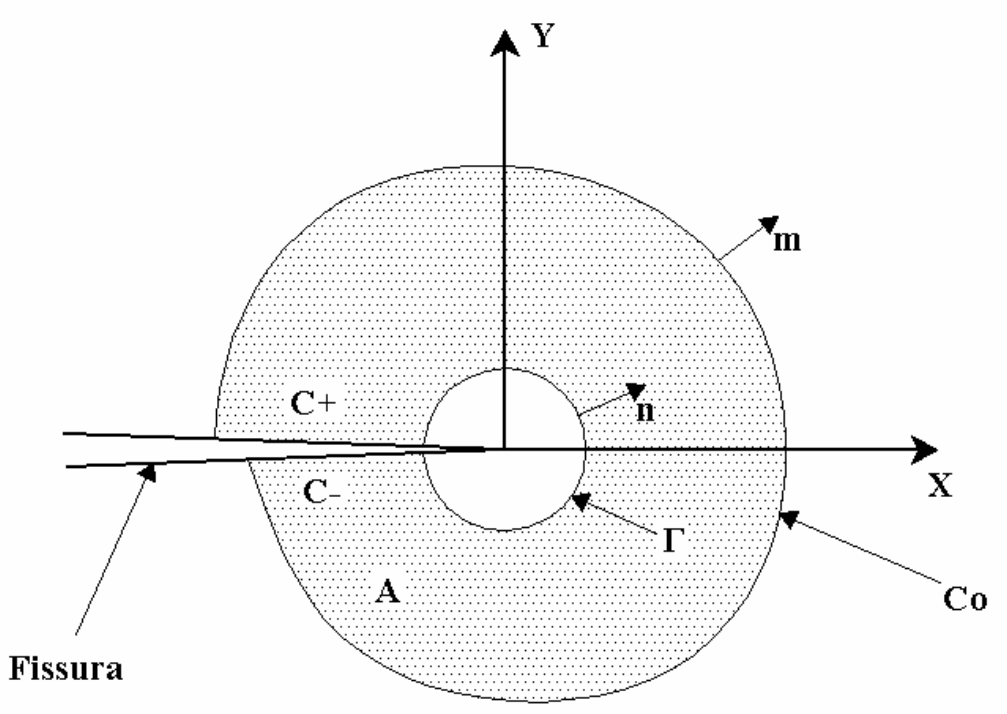

Figura 1.4 - Convenções na ponta da fissura. Domínio A contornado por $\Gamma, \mathrm{C}+, \mathrm{C}-, \mathrm{Co}$. Vetores normais $\mathrm{m}=\mathrm{n}$ em $\mathrm{C}+, \mathrm{C}$ - e Co e $\mathrm{m}=-\mathrm{n}$ em $\Gamma$ (MORAN; SHIH, 1987b).

No contorno mais próximo à ponta da fissura o valor da função ponderadora é unitário, enquanto que no contorno mais externo o valor é nulo. Para qualquer função suficientemente suave, a formulação IDE é válida. Segundo Li, Shih e Needleman (1985), a técnica IDE torna a avaliação numérica da integral-J mais precisa e melhor compatível com o MEF, sob o ponto de vista de implementação.

A conversão de integral de contorno em integral de domínio é bastante atrativa com relação à utilização do MEF. Todas as implementações utilizadas para o cálculo 
das integrais de domínio estão prontamente disponibilizadas em uma análise utilizando o MEF.

Um estudo comparativo entre o método da integral-J e sua respectiva formulação utilizando a técnica IDE foi apresentada por Li, Shih e Needleman (1985). Nesse trabalho, a técnica IDE foi interpretada como uma aplicação do princípio dos trabalhos virtuais e associada ao método da extensão virtual da fissura. Segundo Li, Shih e Needleman (1985) a implementação da técnica IDE, utilizando o MEF, corresponde ao método da extensão virtual da fissura. A análise comparativa dos métodos sugere a utilização de elementos finitos singulares apropriados na região da ponta da fissura e a aplicação da técnica IDE no cálculo da taxa de energia disponibilizada para a propagação da fissura ( $\mathscr{G})$.

\subsubsection{Formulação de Moran e Shih}

Para uma extensa classe de problemas em mecânica da fratura, os parâmetros que caracterizam os campos na ponta da fissura podem ser representados em termos de integrais de contorno ou de domínio. Esses parâmetros são valores críticos os quais podem formar a base de um critério de fratura.

Os resultados satisfatórios de aplicações baseadas na integral-J têm estimulado atividades relacionadas com o desenvolvimento de novas integrais de caminho independente para solucionar problemas de mecânica da fratura (MORAN; SHIH 1987a). Moran e Shih (1987a) mostraram que as integrais de contorno ou domínio independentes, desenvolvidas recentemente, são apenas representações alternativas de integrais de ponta de fissura específicas.

Moran e Shih (1987a, b) apresentaram uma formulação unificada para as integrais de contorno que são utilizadas na obtenção de parâmetros de ponta de fissura em diversos tipos de problemas e suas respectivas formulações equivalentes no domínio. As integrais de contorno foram desenvolvidas sob o ponto de vista de que suas formulações, em quaisquer tipos de problema, são simplesmente obtidas de leis apropriadas de balanço de energia. A utilização das equações de balanço de energia, de restrições apropriadas com relação ao comportamento dos materiais e movimentos na ponta da fissura conduzem diretamente às integrais que são utilizadas em análise de fissuras. 


\subsubsection{Problemas que envolvem modos mistos}

A principal desvantagem dos métodos baseados na integral-J é a necessidade de procedimentos auxiliares para a extração dos fatores de intensidade de tensão em problemas que envolvem modos mistos. Entre os procedimentos de extração mais utilizados citam-se: o método direto, o método da decomposição e o método da integral de interação (MORAN; SHIH, 1987a, b; NAHTA; MORAN, 1993; ORGAN, 1996; RAJU; SHIVAKUMAR, 1990).

Um estudo comparativo entre o método direto e o método da decomposição, aplicado a problemas bidimensionais, foi apresentado por Raju e Shivakumar (1990).

\section{Método direto}

O método direto envolve a avaliação numérica de integrais de linha na vizinhança da ponta da fissura. As integrais de linha utilizadas no método direto são a integral-J e a integral do produto (equação (1.3)). Essas duas integrais, independentes do caminho de integração, definem o fluxo total de energia que passa através do contorno em volta da ponta da fissura nas direções paralela e perpendicular à fissura, respectivamente (Figura 1.3). As parcelas do fluxo de energia relativas a cada modo de deformação são descritas em função das integrais J e do produto

$$
\begin{aligned}
& \mathrm{J}_{\mathrm{x}_{1}}=\mathrm{J}_{\mathrm{I}}+\mathrm{J}_{\mathrm{II}} \\
& \mathrm{J}_{\mathrm{x}_{2}}=-2 \sqrt{\mathrm{J}_{\mathrm{I}} \mathrm{J}_{\mathrm{II}}}
\end{aligned}
$$

sendo $\mathrm{J}_{\mathrm{I}}$ e $\mathrm{J}_{\mathrm{II}}$ as parcelas do fluxo de energia relativo aos modos de deformação I e II (Figuras 2.1 e 2.2), respectivamente. As parcelas $\mathrm{J}_{\mathrm{I}}$ e $\mathrm{J}_{\mathrm{II}}$ podem ser escritas em função das integrais $\mathrm{J}$ e do produto (equação (1.7)).

$$
\begin{aligned}
& \mathrm{J}_{\mathrm{I}}=\frac{1}{4}\left(\sqrt{\mathrm{J}_{\mathrm{x}_{1}}-\mathrm{J}_{\mathrm{x}_{2}}}+\sqrt{\mathrm{J}_{\mathrm{x}_{1}}+\mathrm{J}_{\mathrm{x}_{2}}}\right)^{2} \\
& \mathrm{~J}_{\mathrm{II}}=\frac{1}{4}\left(\sqrt{\mathrm{J}_{\mathrm{x}_{1}}-\mathrm{J}_{\mathrm{x}_{2}}}-\sqrt{\mathrm{J}_{\mathrm{x}_{1}}+\mathrm{J}_{\mathrm{x}_{2}}}\right)^{2} \\
& \mathrm{~J}_{\text {total }}=\mathrm{J}_{\mathrm{I}}+\mathrm{J}_{\mathrm{II}}
\end{aligned}
$$

Com isso, é possível definir quanto cada modo de deformação influencia no fluxo de energia próximo a ponta da fissura e, conseqüentemente, os fatores de intensidade de tensão de cada modo. 
Uma das desvantagens desse método é a dificuldade de avaliar numericamente as integrais de linha próximas a ponta da fissura. Outra desvantagem é que para modos puros os valores das integrais de contorno $\mathrm{J}_{\mathrm{x}_{1}}$ e $\mathrm{J}_{\mathrm{x}_{2}}$ não determinam completamente os modos individuais. Isto ocorre porque no caso em que $\mathrm{J}_{\mathrm{x}_{2}} \equiv 0, \mathrm{~J}_{\mathrm{x}_{1}}$ fornece a energia total. A equação (1.7) sugere que apenas $\mathrm{J}_{\mathrm{I}}$ existe. Essa dificuldade se deve à escolha do sinal positivo para os termos de raiz quadrada na equação (1.7) (RAJU; SHIVAKUMAR, 1990).

\section{Método da decomposição}

O método da decomposição envolve a separação dos campos de deslocamento e tensão em parcelas simétrica e anti-simétrica. Nesse método, a integral do fluxo de energia próxima a ponta da fissura é avaliada apenas no domínio. A parcela simétrica da integral está relacionada com o modo de deformação I e a anti-simétrica com o modo de deformação II.

Obviamente esse método envolve um procedimento adicional. É necessário decompor os campos em componentes simétricos e anti-simétricos. Entretanto, os modos puros são diretamente avaliados.

Segundo Raju e Shivakumar (1990), a aplicação do método direto não apresenta valores precisos tendo em vista a dificuldade de avaliação numérica precisa das integrais de contorno na ponta da fissura. Os valores obtidos com o método da decomposição são condizentes com os encontrados na literatura. O trabalho também apresenta uma análise do comportamento da solução do método IDE utilizando domínios com mais de uma camada de elementos em torno da ponta da fissura e funções de ponderação diferentes. Os resultados mostram que a utilização de apenas uma camada de elementos, em volta da ponta da fissura, e funções de ponderação lineares são suficientes para obter valores precisos da taxa de energia disponibilizada para a propagação da fissura $(\mathscr{G})$.

\section{Método da integral de interação}

O método da integral de interação envolve a utilização de campos auxiliares assintóticos na região da ponta da fissura. Esse método foi desenvolvido com base na forma unificada da integral de caminho independente apresentada por Moran e Shih (1987a, b). Dentre os métodos citados, esse é o mais geral para extração dos fatores de intensidade de tensão. Os campos auxiliares devem satisfazer as equações que regem o problema e podem ser superpostos aos campos atuais. A introdução desses campos 
auxiliares no sistema resulta em termos de interação na expressão da taxa de energia disponibilizada para a propagação da fissura, os quais podem ser utilizados para separar as parcelas da taxa de energia disponibilizada referentes a cada modo. A integral de interação resultante é posteriormente transformada de integral de contorno para integral de domínio. Com a escolha prudente dos campos auxiliares, os fatores de intensidade de tensão, relativos a cada um dos modos, podem ser extraídos diretamente.

Nahta e Moran (1993) enfatizaram a aplicação do método IDE para a extração dos fatores de intensidade de tensão em modos mistos. Aplicaram o método da integral de interação para problemas axisimétricos de fissuras em interface entre materiais.

Organ (1996) utilizou o método da integral de interação para determinar os fatores de intensidade de tensão em problemas de fratura dinâmica envolvendo modos mistos.

Gosz, Dolbow e Moran (1998) utilizaram o mesmo método na avaliação dos fatores de intensidade de tensão ao longo de fissuras curvas tridimensionais em interface entre materiais.

Os valores obtidos nas análises com o método da integral de interação são condizentes com a literatura. Em todos os casos, os valores obtidos para os fatores de intensidade de tensão apresentaram boa precisão numérica.

\subsubsection{Métodos de extração superconvergentes}

Antes de iniciar a apresentação dos métodos superconvergentes são necessárias algumas definições. A energia potencial total de um corpo elástico, submetido a ações externas, pode ser escrita da seguinte forma (SZABÓ; BABUŠKA, 1991):

$$
\Pi(\mathrm{u})=\frac{1}{2} \mathrm{~B}(\mathrm{u}, \mathrm{u})-\mathrm{L}(\mathrm{u})
$$

sendo $\frac{1}{2} B(u, u)$ a energia de deformação e $L(u)$ o trabalho das forças externas. B(.,.) é uma forma bilinear e L(.) é uma forma linear. Com isso, define-se a norma energia:

$$
\|\mathrm{u}\|_{E(\Omega)}=\sqrt{\frac{1}{2} \mathrm{~B}(\mathrm{u}, \mathrm{u})}
$$


Os métodos citados nesta seção são ditos superconvergentes porque convergem para os valores esperados tão rápido quanto a energia de deformação.

Babuška e Miller (1984a, b, c) apresentaram uma metodologia para o cálculo de funcionais de soluções do método de elementos finitos. O funcional pode ser, por exemplo, uma componente de tensão em um ponto ou um fator de intensidade de tensão. Seja tal funcional denotado por $\Phi\left(\mathrm{u}_{\mathrm{ef}}\right)$ sendo $\mathrm{u}_{\mathrm{ef}}$ a solução numérica de um problema de valor de contorno obtida com o MEF. Por meio de uma expressão do tipo

$$
\Phi\left(\mathrm{u}_{\mathrm{ef}}\right)=\mathrm{B}\left(\mathrm{u}_{\mathrm{ef}}, \mathrm{v}\right)
$$

o valor do funcional é calculado. B(.,.) é uma forma bilinear e $\mathrm{v}$ é uma função adequadamente escolhida e denominada de função extratora. Métodos desse tipo são chamados de métodos de extração. Babuška e Miller (1984c) demonstraram matematicamente que o erro no valor do funcional calculado por tais métodos pode ser escrito como

$$
\left|\Phi\left(\mathrm{u}_{\text {exata }}-\mathrm{u}_{\text {ef }}\right)\right| \leq\left\|\mathrm{u}_{\text {exata }}-\mathrm{u}_{\text {ef }}\right\|_{\mathrm{E}(\Omega)}\left\|\mathrm{w}_{\text {exata }}-\mathrm{w}_{\text {ef }}\right\|_{\mathrm{E}(\Omega)}
$$

sendo \|\|$_{E(\Omega)}$ a norma energia (1.9), $\mathrm{u}_{\text {exata }}$ a solução exata do problema, $\mathrm{w}_{\text {ef }}$ e $\mathrm{w}_{\text {exata }}$ as soluções de elementos finitos e exata, respectivamente, de um problema auxiliar (utilizado apenas para demonstrações teóricas). Babuška e Miller (1984c) também demonstraram que é possível selecionar a função extratora $v$ de tal forma que

$$
\left\|\mathrm{W}_{\text {exata }}-\mathrm{W}_{\text {ef }}\right\|_{\mathrm{E}(\Omega)} \rightarrow 0
$$

tão quanto ou mais rápido do que

$$
\left\|\mathrm{u}_{\text {exata }}-\mathrm{u}_{\mathrm{ef}}\right\|_{\mathrm{E}(\Omega)} \rightarrow 0
$$

com o aumento do número de graus de liberdade da solução de elementos finitos. Portanto, os valores dos funcionais calculados por esses métodos de extração possuem pelo menos a mesma taxa de convergência da energia de deformação do problema, ou seja, os valores do funcional são superconvergentes (BABUŠKA; MILLER, 1984b, c). 


\subsubsection{Os métodos MIC e MFC}

Szabó e Babuška (1988) apresentaram a base teórica de dois métodos utilizados para extração dos fatores de intensidade de tensão da solução do MEF: o método da integral de contorno (MIC) e o método da função cutoff (MFC). Nessa seção, os métodos desenvolvidos por Szabó e Babuška (1988) serão descritos sucintamente. Nos Capítulos 3 e 4, as formulações dos métodos MIC e MFC serão apresentadas com mais detalhes.

Ambos os métodos possuem a formulação baseada no Princípio dos Trabalhos Virtuais (PTV). Aplicando-se o teorema de Green na formulação obtida com o PTV é possível chegar a Lei de Betti que consiste em uma integral de caminho independente. O caminho de integração para extrair os fatores de intensidade de tensão deve iniciar em uma aresta da fissura e terminar na outra e o sentido da integração deve ser anti-horário em relação à fissura (Figura 1.5 - para o caso de fissura: $\alpha=2 \pi$ ).

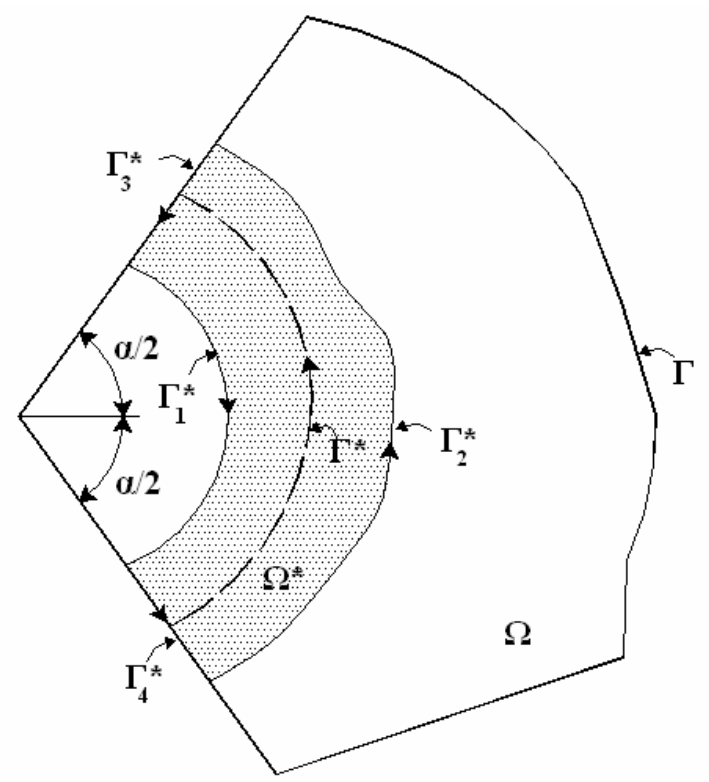

Figura 1.5 - Caminho de integração em torno de um vértice do domínio (MIC e MFC) (SZABÓ; BABUŠKA, 1991).

Utilizando um caminho de integração circular e a solução exata do problema, o raio utilizado para extração dos fatores de intensidade pode ser arbitrário. Porém, como a solução do MEF é utilizada na extração, o raio de integração deve ser próximo à extremidade da fissura para garantir a independência do caminho.

A formulação para o MIC e para o MFC possui um caráter geral com relação ao tipo de singularidade presente no domínio. A singularidade é caracterizada pelo ângulo 
existente entre as arestas que compõem uma região de extremidade no domínio (Figura 1.5). Ambos os métodos podem ser utilizados na extração dos fatores $K_{I}, K_{I I}$ e $K_{I I I}$, bem como outros coeficientes da expansão assintótica da solução em torno de uma aresta. A diferença existente entre esses métodos está no tipo de função extratora utilizada.

No MIC, a função extratora possui a mesma forma da solução analítica do campo de deslocamento nas extremidades reentrantes presentes no domínio, utilizando os autovalores negativos.

No MFC utiliza-se a mesma forma da função extratora do MIC multiplicada por uma função suave, denominada de função cutoff, que varia de 1, no contorno mais próximo a fissura, a 0, no contorno mais externo (Figura 1.5). Processo semelhante ao utilizado no método IDE.

O MFC modifica a função de extração tal que (SZABÓ; BABUŠKA, 1988):

- O caráter local da função de extração é preservado no vértice (conseqüentemente a função cutoff é igual a 1 no contorno interno)

- A função cutoff é uma função suave tal que a solução do problema auxiliar pode ser bem aproximada pela solução do MEF.

- A função extratora do MFC e a sua derivada anulam-se no contorno e fora do caminho fechado de integração, conseqüentemente as tensões e trações correspondentes para a solução do MEF não precisam ser consideradas. Por esta razão o MFC é mais preciso do que o MIC.

Szabó e Babuška (1988) também apresentaram uma análise da convergência dos fatores de intensidade de tensão obtidos com MIC e MFC da solução do MEF na versão $\mathrm{p}^{1}$ em problemas bidimensionais. Em todos os casos os métodos convergem para os valores analíticos na mesma taxa da energia de deformação, ou mais rápido. Porém a convergência não é, em geral, monotônica. Pela precisão obtida para os fatores de intensidade de tensão, um destaque maior é dado aos valores extraídos com o método MFC.

\footnotetext{
${ }^{1}$ A versão p do MEF é uma extensão do MEF baseada no controle do erro da solução por meio do incremento da ordem polinomial das funções de forma dos elementos. Denomina-se extensão de um método numérico toda mudança sistemática de discretização que gera um aumento no número de graus de liberdade da solução. Outras extensões do MEF são: a versão h (que envolve a variação do número de graus de liberdade pelo controle do tamanho dos elementos) e a versãp hp (que é uma combinação das versões h e p) (SZABÓ e BABUŠKA, 1991).
} 
Szabó e Babuška (1988) mostraram uma análise da convergência da norma do vetor cujas coordenadas são os fatores de intensidade de tensão. Nessa análise foram utilizados os métodos MIC, MFC e o método da derivada da rigidez (PARKS, 1974). Em todos os métodos, os resultados foram obtidos a partir da solução do MEF na versão $\mathrm{p}^{1}$ Os três métodos apresentaram forte convergência e mais uma vez o MFC apresentou os melhores resultados. Nessa análise, o método da derivada da rigidez apresentou um maior custo computacional do que o MIC e o MFC.

Algumas complicações podem surgir com o uso e implementação do MIC e do MFC em problemas cujo comportamento da solução na região de singularidade não é conhecido. Isso dificulta a escolha da função extratora que será adotada na implementação. As funções extratoras utilizadas no MIC e no MFC dependem dos autovalores e das autofunções do problema. Em problemas mais gerais é possível obter os autovalores e as autofunções numericamente (PAPADAKIS; BABUŠKA, 1995; YOSIBASH; SZABÓ, 1995b).

Yosibash e Szabó (1995c) apresentaram uma extensão do MIC para extrair os fatores de intensidade de tensão em problemas axisimétricos utilizando a versão $p$ do $\mathrm{MEF}^{1}$. Embora o problema axisimétrico possua a formulação reduzida para o problema bidimensional, faz-se necessário um tratamento especial para encontrar sua solução na vizinhança de arestas de singularidade. Sendo assim, utilizou-se o Método de Steklov Modificado (MSM) (YOSIBASH; SZABÓ, 1995b) para compor a função extratora desta extensão do MIC. Segundo Yosibash e Szabó (1995c), os valores computados com a extensão do MIC para problemas axisimétricos também exibem superconvergência.

\subsubsection{Método de Yosibash e Szabó}

Yosibah e Szabó (1995b) desenvolveram o Método de Steklov Modificado (MSM). Esse método pode ser aplicado a problemas com singularidades associadas a domínios que possuem extremidade, anisotropia, interface de materiais compostos e mudanças bruscas nas condições de contorno. Exemplos numéricos de problemas isotrópicos e anisotrópicos, utilizando a versão $\mathrm{p}$ do $\mathrm{MEF}^{1}$, comprovaram que o MSM exibe superconvergência. Esse método pode ser utilizado na elaboração de funções extratoras para os métodos MIC e MFC aplicados aos casos em que a solução exata não é conhecida. 
Utilizando o MSM para compor funções extratoras e a versão p do MEF, Yosibash e Szabó (1995a, 1996) desenvolveram um método baseado na formulação fraca complementar para problemas elípticos. Baseado no mesmo princípio, Yosibash (1997b) desenvolveu um método para a extração das componentes do tensor de tensão em um ponto, da solução do MEF nas versões $\mathrm{h}$ e $\mathrm{p}^{1}$, para problemas bidimensionais elásticos lineares.

A comparação numérica entre o método de Yosibash e Szabó (1995a, 1996) e o MIC mostra que, para a versão $\mathrm{p}$ do $\mathrm{MEF}$, os dois métodos convergem fortemente para os valores esperados. Porém, os valores obtidos com o MIC apresentaram melhor convergência. Segundo Yosibash e Szabó (1995a, 1996), o melhor rendimento do MIC, em relação ao método baseado na formulação fraca complementar, está relacionado ao uso da função extratora com base na solução do MSM, enquanto que no MIC foi utilizada a solução analítica.

Yosibash (1997d, 1998) apresentou uma aplicação do método baseado na formulação fraca complementar, em conjunto com o MSM e a versão p do MEF, para problemas termoelásticos bidimensionais com pontos de singularidade. Yosibash (1997a, c) ampliou a formulação do MSM para problemas tridimensionais elásticos lineares com arestas de singularidade. Nos dois casos, os valores obtidos são condizentes com os encontrados na literatura.

Os autovalores e autofunções, obtidos com o MSM, podem ser utilizados em extensões, para o caso tridimensional, dos métodos de extração dos fatores de intensidade de tensão descritos nessa seção. Particularmente o MIC e o MFC, que são os métodos de extração a serem utilizados no presente trabalho.

O presente trabalho propõe a extração dos fatores de intensidade de tensão da solução do método de elementos finitos generalizados (MEFG). Os problemas apresentados aqui são elásticos bidimensionais, lineares e isotrópicos. Na extração dos fatores de intensidade de tensão utilizam-se os métodos da integral de contorno (MIC) e da função cutoff (MFC) em problemas de modos mistos e o método da integral-J em problemas de modos puros. 


\subsection{Estrutura da dissertação}

Nesta seção, apresentam-se alguns parâmetros e aplicações da Mecânica da Fratura, uma breve introdução sobre o MEFG, os objetivos do presente trabalho e uma revisão sobre os principais métodos encontrados na literatura. Nas demais seções, a dissertação encontra-se divida da seguinte forma:

- No Capítulo 2, desenvolve-se a formulação analítica para a solução da elasticidade na vizinhança de regiões reentrantes do domínio. Tal solução tem grande importância na formulação dos métodos de extração MIC e MFC;

- No Capítulo 3, apresenta-se a formulação dos métodos de extração implementados para as análises desenvolvidas nesta dissertação;

- No Capítulo 4, propõe-se uma formulação numérica para os métodos de extração que possui como principal característica a independência da discretização utilizada na análise. No processo de integração numérica, os elementos da malha não são utilizados;

- O Capítulo 5 exibe uma descrição do programa SET (Scientific and Engineering Toolkit), da implementação computacional dos métodos de extração e dos recursos computacionais utilizados nas implementações;

- O Capítulo 6 expõe os resultados e as discussões dos exemplos numéricos desenvolvidos no presente trabalho;

- E, finalmente, o Capítulo 7 apresenta as principais conclusões deste estudo e as propostas para trabalhos futuros.

Nos apêndices A, B e C encontram-se algumas formulações desenvolvidas para a implementação dos métodos de extração, bem como os códigos computacionais utilizados nesses desenvolvimentos. 


\section{Problemas elásticos com pontos singulares}

Neste capítulo, apresenta-se a solução da elasticidade para problemas bidimensionais que possuem regiões reentrantes em seu domínio. Nesse conjunto de problemas encontra-se o objeto de estudo da mecânica da fratura elástica linear: o problema de uma fissura em um domínio elástico. As formulações apresentadas aqui são particularizadas para o caso plano e material isotrópico e estão baseadas em (BROEK, 1982; SZABÓ; BABUŠKA, 1991; VALLIAPAN, S. 1981).

\subsection{Conceitos básicos de Mecânica da Fratura}

A solução elástica linear da fratura é um caso particular da solução da elasticidade para problemas que possuem arestas reentrantes em seu domínio. Nessa seção, apresenta-se um breve resumo dos principais parâmetros da mecânica da fratura. Tais parâmetros são necessários para o entendimento e desenvolvimento da solução da elasticidade na vizinhança da fissura.

\subsubsection{Modos de deformação}

O campo de deformação em torno de uma fissura em um sólido qualquer, submetido a um determinado carregamento, pode ser decomposto em três componentes ou modos de deformação (BROEK, 1982). Tais modos são definidos a seguir:

Modo de abertura (Modo I)

Modo de deformação em que as duas faces da fissura são separadas na direção y (Figura 2.1) e as deformações são simétricas em relação aos planos x-z e x-y. Para esse 
modo, os deslocamentos da superfície da fissura são sempre perpendiculares ao plano da fissura.

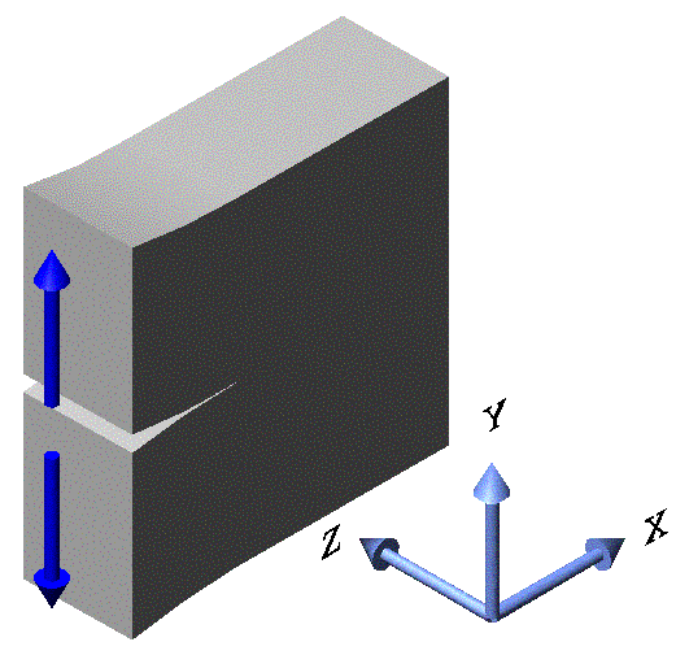

Figura 2.1 - Modo de abertura (Modo I).

\section{Modo de cisalhamento ou de deslizamento (Modo II)}

Modo de deformação em que as faces da fissura deslizam uma sobre a outra na direção x (Figura 2.2) e as deformações são simétricas em relação ao plano x-y e antisimétrica em relação ao plano x-z. Nesse caso, os deslocamentos da superfície da fissura acontecem no plano da fissura e perpendiculares à aresta da extremidade da fissura.

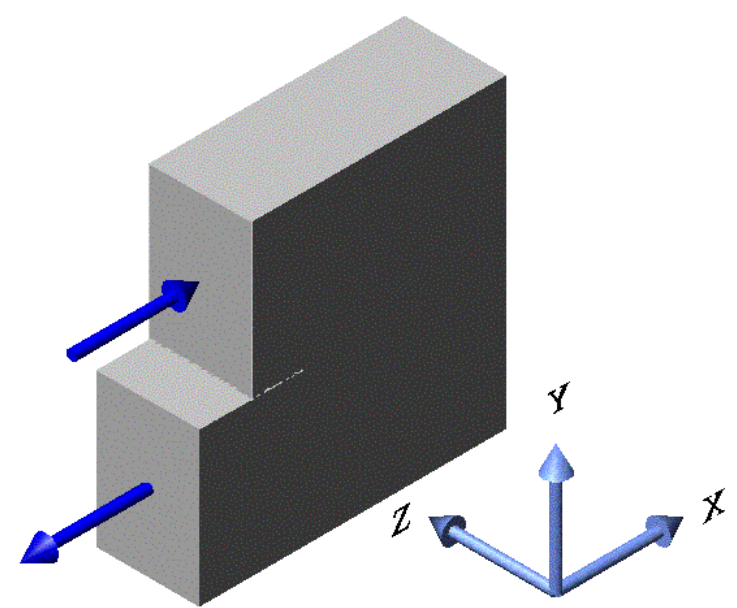

Figura 2.2 - Modo de cisalhamento (Modo II).

\section{Modo de rasgamento (Modo III)}

Modo de deformação em que as faces da fissura deslizam uma sobre a outra na direção z (Figura 2.3) e as deformações são anti-simétricas em relação aos planos x-y e 
x-z. Nesse caso, os deslocamentos da superfície da fissura acontecem no plano da fissura e paralelos à aresta da extremidade da fissura.

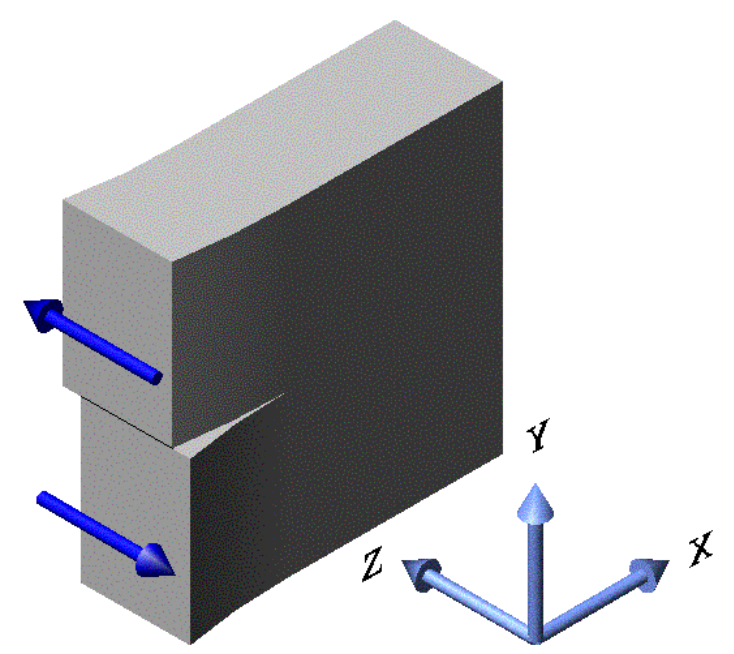

Figura 2.3 - Modo de rasgamento (Modo III).

A deformação da fissura pode ser representada por uma superposição dos três modos de deformação apresentados. Para o caso plano, a resposta de deformação da fissura é composta por modos I e II. O modo III aparece apenas nos casos tridimensionais.

\subsubsection{Fatores de intensidade de tensão}

Cada modo de deformação possui um campo de tensão associado. A Equação que descreve os campos de tensão na vizinhança de uma fissura reta pode ser escrita da seguinte forma (BROEK, 1982):

$$
\sigma_{\mathrm{ij}}^{(\mathrm{n})}=\frac{\mathrm{K}_{\mathrm{n}}}{\sqrt{2 \pi \mathrm{r}}} \mathrm{f}_{\mathrm{ij}}^{(\mathrm{n})}(\theta)
$$

$\mathrm{n}=\mathrm{I}$, II, III indica cada um dos modos de deformação, $\mathrm{r}$ e $\theta$ são coordenadas polares centradas na ponta da fissura e $\mathrm{f}_{\mathrm{ij}}^{(\mathrm{n})}(\theta)$ são funções suaves diferentes para cada componente do tensor de tensão e para cada modo.

Os parâmetros $\mathrm{K}_{\mathrm{I}}, \mathrm{K}_{\mathrm{II}}$ e $\mathrm{K}_{\mathrm{III}}$ são conhecidos como fatores de intensidade de tensão correspondentes aos três modos de deformação. Esses fatores caracterizam a magnitude dos campos de tensão na ponta da fissura. 


\subsection{Descrição da solução exata em um domínio com pontos singulares}

Para problemas bidimensionais, a solução exata pode ser escrita como a soma de duas funções: uma função suave qualquer $u_{1}$ e, na vizinhança de um número finito de pontos $\mathrm{P}_{1}, \mathrm{P}_{2}, \ldots, \mathrm{P}_{\mathrm{n}}$, funções na forma (SZABÓ; BABUŠKA, 1991):

$$
\mathrm{u}_{2}=\sum_{\mathrm{i}=1}^{\mathrm{M}_{\mathrm{k}}} \mathrm{A}_{\mathrm{i}} \mathrm{r}^{\lambda_{\mathrm{i}}} \Psi_{\mathrm{i}}(\theta)
$$

sendo $\mathrm{r}$ e $\theta$ coordenadas polares centradas no ponto $\mathrm{P}_{\mathrm{k}}(\mathrm{k}=1,2, \ldots, \mathrm{n}), \mathrm{A}_{\mathrm{i}}$ coeficientes que dependem do carregamento e da geometria do domínio e $\Psi_{\mathrm{i}}(\theta)$ funções suaves.

Os pontos $\mathrm{P}_{\mathrm{k}}$ são denominados de pontos singulares, os quais podem aparecer no interior do domínio de solução, no contorno do domínio de solução, ou fora do domínio de solução. Um típico domínio de solução com pontos singulares é mostrado na Figura 2.4.

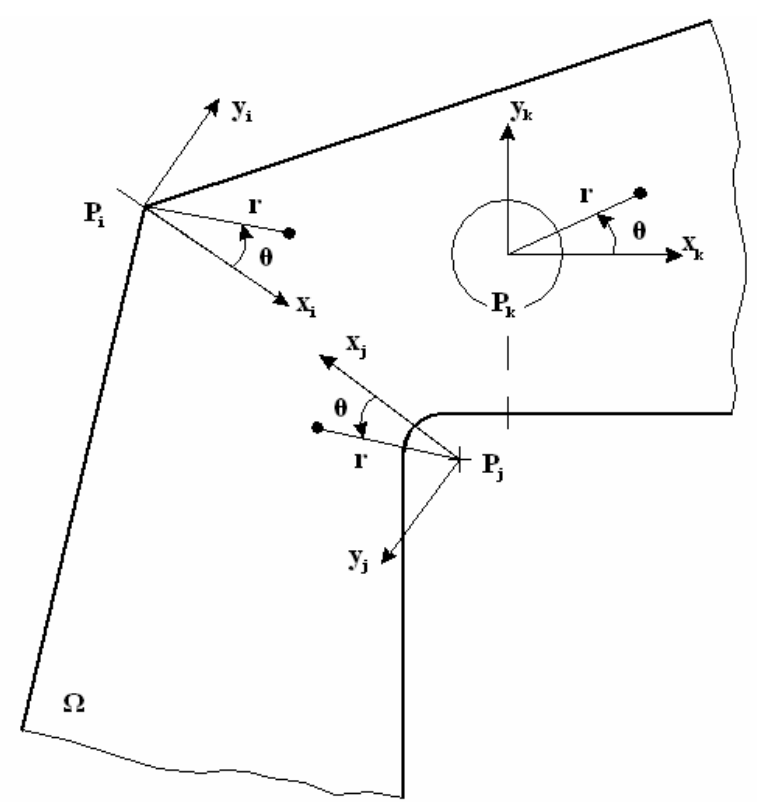

Figura 2.4 - Domínio típico com pontos singulares (SZABÓ; BABUŠKA, 1991).

Para pontos singulares que pertencem ao domínio de solução, ou no contorno do domínio de solução, o parâmetro $\lambda_{i}$ e as funções associadas $\Psi_{i}(\theta)$ caracterizam a singularidade da solução exata $\left(\mathrm{u}_{\mathrm{EX}}\right)$ na vizinhança do ponto singular. Em pontos 
singulares que não pertencem ao domínio de solução, a distância do ponto singular ao domínio de solução, junto ao parâmetro $\lambda_{\mathrm{i}}$ e às funções associadas $\Psi_{\mathrm{i}}(\theta)$, determinam a singularidade de $\mathrm{u}_{\mathrm{EX}}$.

\subsection{Solução exata bidimensional na vizinhança de pontos singulares}

A solução de problemas elásticos bidimensionais, considerando forças de volume nulas ou constantes, resume-se a encontrar uma solução para a equação (2.3)

$$
\left(\frac{\partial^{2}}{\partial \mathrm{r}^{2}}+\frac{1}{\mathrm{r}} \frac{\partial}{\partial \mathrm{r}}+\frac{1}{\mathrm{r}^{2}} \frac{\partial^{2}}{\partial \theta^{2}}\right)\left(\frac{\partial^{2}}{\partial \mathrm{r}^{2}}+\frac{1}{\mathrm{r}} \frac{\partial}{\partial \mathrm{r}}+\frac{1}{\mathrm{r}^{2}} \frac{\partial^{2}}{\partial \theta^{2}}\right) \Phi=0
$$

conhecida como equação biharmônica, sendo $\Phi$ denominada função de tensão de Airy (VALLIAPAN, 1981).

$\mathrm{Na}$ vizinhança de quinas reentrantes (Figura 2.5), a função de tensão pode ser descrita na forma

$$
\Phi=\Phi(\mathrm{r}, \theta)=\mathrm{r}^{\lambda+1} \mathrm{~F}(\theta)
$$

A escolha da função de tensão apresentada em (2.4) é conhecida como técnica de separação de variáveis.

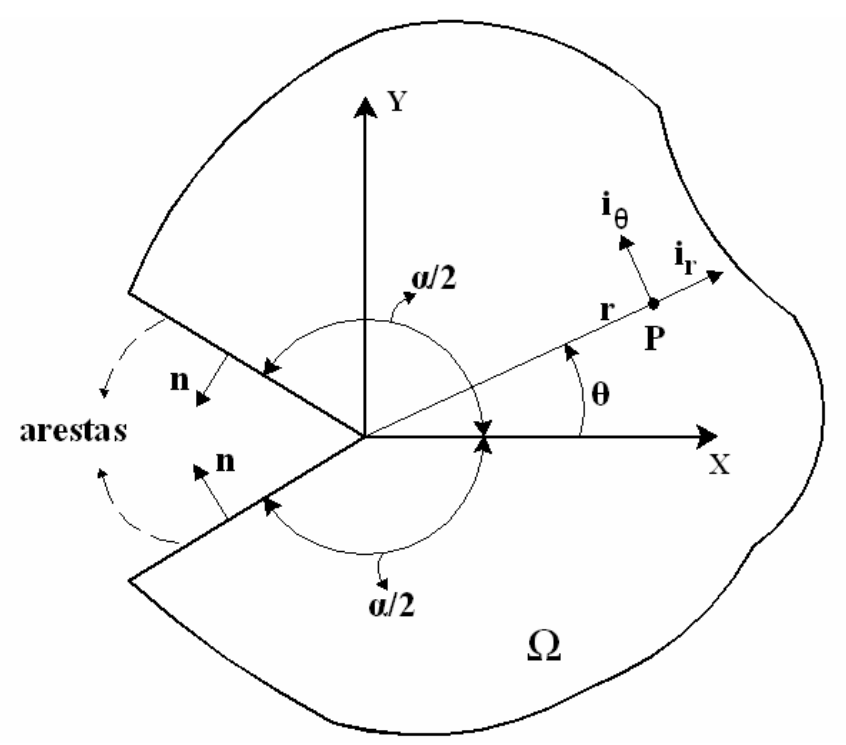

Figura 2.5 - Vizinhança da singularidade (SZABÓ; BABUŠKA, 1991). 
Para que a equação (2.4) satisfaça a equação biharmônica (2.3), $F(\theta)$ deve obedecer a seguinte equação:

$$
\frac{d^{4} F(\theta)}{d^{4} \theta}+2\left(\lambda^{2}+1\right) \frac{d^{2} F(\theta)}{d^{2} \theta}+\left(\lambda^{2}+1\right)^{2} F(\theta)=0
$$

A solução geral da equação (2.5), para $\lambda \neq 0$ e $\lambda \neq \pm 1$ é escrita na forma:

$$
\mathrm{F}(\theta)=\underbrace{\mathrm{a}_{1} \cos [(\lambda-1) \theta]+\mathrm{a}_{2} \cos [(\lambda+1) \theta]}_{\text {Parte simética (PAR) }}+\underbrace{\mathrm{a}_{3} \operatorname{sen}[(\lambda-1) \theta]+\mathrm{a}_{4} \operatorname{sen}[(\lambda+1) \theta]}_{\text {Parte anti-simétrica (ÍMPAR) }} .
$$

A relação entre as componentes de tensão em coordenadas polares $\left(\sigma_{\mathrm{r}}, \sigma_{\theta}, \tau_{\mathrm{r} \theta}\right)$ e a função de tensão $\Phi$ é da forma (VALLIAPAN, 1981):

$$
\begin{aligned}
& \sigma_{\mathrm{r}}=\frac{1}{\mathrm{r}} \frac{\partial \Phi}{\partial \mathrm{r}}+\frac{1}{\mathrm{r}^{2}} \frac{\partial^{2} \Phi}{\partial \mathrm{r}^{2}}=\mathrm{r}^{\lambda-1}\left[(\lambda+1) \mathrm{F}(\theta)+\frac{\mathrm{d}^{2} \mathrm{~F}(\theta)}{\mathrm{d} \theta^{2}}\right] \\
& \sigma_{\theta}=\frac{\partial^{2} \Phi}{\partial \mathrm{r}^{2}}=\lambda(\lambda+1) \mathrm{r}^{\lambda-1} \mathrm{~F}(\theta) \\
& \tau_{\mathrm{r} \theta}=-\frac{1}{\mathrm{r}} \frac{\partial^{2} \Phi}{\partial \mathrm{r} \partial \theta}+\frac{1}{\mathrm{r}^{2}} \frac{\partial \Phi}{\partial \theta}=-\lambda \mathrm{r}^{\lambda-1} \frac{\mathrm{dF}(\theta)}{\mathrm{d} \theta}
\end{aligned}
$$

Para determinar os autovalores $\lambda$ e as constantes $a_{i}, i=1,2,3,4$, assume-se que o domínio $\Omega$ (Figura 2.5) é infinito e impõe-se apenas as condições de contorno nas arestas. Consideraremos aqui o caso em que as tensões nas arestas em $\theta= \pm \alpha / 2$ (Figura 2.5) são nulas. O procedimento para outras condições de contorno nas arestas é análogo. Para $\theta=-\alpha / 2$ o vetor normal a aresta é $n=-e_{\theta}$. Assim, aplicando a condição de contorno na aresta $\theta=-\alpha / 2$, tem-se:

$$
\underset{\approx}{\sigma}\left(\mathrm{r},-\frac{\alpha}{2}\right) \cdot \mathrm{n}=\left[\begin{array}{cc}
\sigma_{\mathrm{r}}\left(\mathrm{r},-\frac{\alpha}{2}\right) & \tau_{\mathrm{r} \theta}\left(\mathrm{r},-\frac{\alpha}{2}\right) \\
\tau_{\mathrm{r} \theta}\left(\mathrm{r},-\frac{\alpha}{2}\right) & \sigma_{\theta}\left(\mathrm{r},-\frac{\alpha}{2}\right)
\end{array}\right]\left\{\begin{array}{c}
0 \\
-1
\end{array}\right\} \rightarrow \begin{aligned}
& \tau_{\mathrm{r} \theta}\left(\mathrm{r},-\frac{\alpha}{2}\right)=0 \\
& \sigma_{\theta}\left(\mathrm{r},-\frac{\alpha}{2}\right)=0
\end{aligned} .
$$

Para $\theta=\alpha / 2$ o vetor normal à aresta é $\mathrm{n}=\mathrm{e}_{\theta}$. Com isso, aplicando-se a condição de contorno na aresta $\theta=\alpha / 2$, tem-se: 


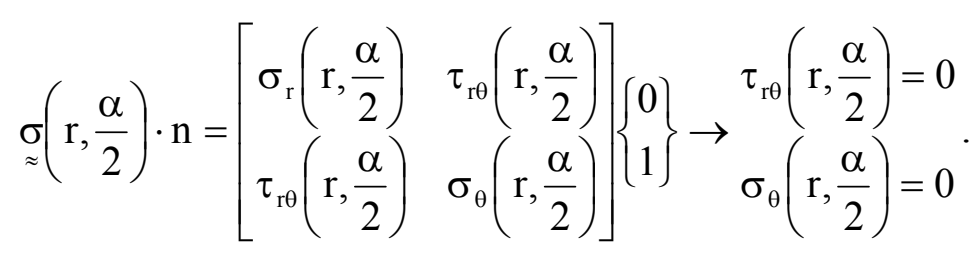

Assim, utilizando as equações (2.6), (2.8), (2.9), tem-se:

$$
\left[\begin{array}{cccc}
\cos \left((\lambda-1) \frac{\alpha}{2}\right) & \cos \left((\lambda+1) \frac{\alpha}{2}\right) & -\operatorname{sen}\left((\lambda-1) \frac{\alpha}{2}\right) & -\operatorname{sen}\left((\lambda+1) \frac{\alpha}{2}\right) \\
\Lambda \operatorname{sen}\left((\lambda-1) \frac{\alpha}{2}\right) & \operatorname{sen}\left((\lambda+1) \frac{\alpha}{2}\right) & \Lambda \cos \left((\lambda-1) \frac{\alpha}{2}\right) & \cos \left((\lambda+1) \frac{\alpha}{2}\right) \\
\cos \left((\lambda-1) \frac{\alpha}{2}\right) & \cos \left((\lambda+1) \frac{\alpha}{2}\right) & \operatorname{sen}\left((\lambda-1) \frac{\alpha}{2}\right) & \operatorname{sen}\left((\lambda+1) \frac{\alpha}{2}\right) \\
\Lambda \operatorname{sen}\left((\lambda-1) \frac{\alpha}{2}\right) & \operatorname{sen}\left((\lambda+1) \frac{\alpha}{2}\right) & -\Lambda \cos \left((\lambda-1) \frac{\alpha}{2}\right) & -\cos \left((\lambda+1) \frac{\alpha}{2}\right)
\end{array}\right]\left(\begin{array}{l}
a_{1} \\
a_{2} \\
a_{3} \\
a_{4}
\end{array}\right)=\left(\begin{array}{l}
0 \\
0 \\
0 \\
0
\end{array}\right)
$$

Manipulando algebricamente o sistema (2.10), chega-se a um sistema equivalente no qual é possível verificar que $\mathrm{a}_{1}$ e $\mathrm{a}_{2}$ são independentes de $\mathrm{a}_{3}$ e $\mathrm{a}_{4}$.

$$
\left[\begin{array}{cccc}
\cos \left((\lambda-1) \frac{\alpha}{2}\right) & \cos \left((\lambda+1) \frac{\alpha}{2}\right) & 0 & 0 \\
\Lambda \operatorname{sen}\left((\lambda-1) \frac{\alpha}{2}\right) & \operatorname{sen}\left((\lambda+1) \frac{\alpha}{2}\right) & 0 & 0 \\
0 & 0 & \operatorname{sen}\left((\lambda-1) \frac{\alpha}{2}\right) & \operatorname{sen}\left((\lambda+1) \frac{\alpha}{2}\right) \\
0 & 0 & \Lambda \cos \left((\lambda-1) \frac{\alpha}{2}\right) & \cos \left((\lambda+1) \frac{\alpha}{2}\right)
\end{array}\right]\left(\begin{array}{l}
a_{1} \\
a_{2} \\
a_{3} \\
a_{4}
\end{array}\right)=\left(\begin{array}{l}
0 \\
0 \\
0 \\
0
\end{array}\right)
$$

sendo:

$$
\Lambda \stackrel{\operatorname{def}}{=} \frac{\lambda-1}{\lambda+1}
$$

Para que a solução não trivial exista, o determinante da matriz do sistema (2.11) deve ser nulo. Para isso, as seguintes equações devem ser satisfeitas:

$$
\cos \left((\lambda-1) \frac{\alpha}{2}\right) \operatorname{sen}\left((\lambda+1) \frac{\alpha}{2}\right)-\Lambda \operatorname{sen}\left((\lambda-1) \frac{\alpha}{2}\right) \cos \left((\lambda+1) \frac{\alpha}{2}\right)=0
$$




$$
\operatorname{sen}\left((\lambda-1) \frac{\alpha}{2}\right) \cos \left((\lambda+1) \frac{\alpha}{2}\right)-\Lambda \cos \left((\lambda-1) \frac{\alpha}{2}\right) \operatorname{sen}\left((\lambda+1) \frac{\alpha}{2}\right)=0
$$

Simplificando:

$$
\begin{aligned}
& \operatorname{sen}(\lambda \alpha)+\lambda \operatorname{sen}(\lambda \alpha)=0, \\
& \operatorname{sen}(\lambda \alpha)-\lambda \operatorname{sen}(\lambda \alpha)=0 .
\end{aligned}
$$

Assumindo que $\lambda$ é um número real, se $\lambda$ é solução da equação (2.15) ou da equação (2.16), então $-\lambda$ também é solução. Entretanto, para que o campo de tensão correspondente à solução possua energia de deformação finita, $\lambda$ deve ser maior que zero $(\lambda>0)$.

Considerando que $\lambda_{i}^{(1)}, i=1,2, \ldots$, é solução da equação (2.15), do sistema (2.11) tem-se:

$$
\begin{gathered}
a_{1} \cos \left(\left(\lambda_{i}^{(1)}-1\right) \frac{\alpha}{2}\right)+a_{2} \cos \left(\left(\lambda_{i}^{(1)}+1\right) \frac{\alpha}{2}\right)=0, \\
a_{1} \Lambda_{i}^{(1)} \operatorname{sen}\left(\left(\lambda_{i}^{(1)}-1\right) \frac{\alpha}{2}\right)+a_{2} \operatorname{sen}\left(\left(\lambda_{i}^{(1)}+1\right) \frac{\alpha}{2}\right)=0,
\end{gathered}
$$

sendo $\Lambda_{i}^{(1)}=\frac{\lambda_{i}^{(1)}-1}{\lambda_{i}^{(1)}+1}$. Assim, define-se:

$$
\mathrm{Q}_{\mathrm{i}}^{(1)}=\frac{\mathrm{a}_{2}}{\mathrm{a}_{1}}=-\frac{\cos \left(\left(\lambda_{\mathrm{i}}^{(1)}-1\right) \frac{\alpha}{2}\right)}{\cos \left(\left(\lambda_{\mathrm{i}}^{(1)}+1\right) \frac{\alpha}{2}\right)}=-\frac{\Lambda_{\mathrm{i}}^{(1)} \operatorname{sen}\left(\left(\lambda_{\mathrm{i}}^{(1)}-1\right) \frac{\alpha}{2}\right)}{\operatorname{sen}\left(\left(\lambda_{\mathrm{i}}^{(1)}+1\right) \frac{\alpha}{2}\right)}
$$

Utilizando-se a definição (2.19), a equação (2.4) pode ser escrita da seguinte forma:

$$
\Phi=\mathrm{r}^{\lambda_{i}^{(1)}+1}\left[\cos \left(\left(\lambda_{i}^{(1)}-1\right) \theta\right)+Q_{i}^{(1)} \cos \left(\left(\lambda_{i}^{(1)}+1\right) \theta\right)\right] .
$$


Considerando $\lambda_{i}^{(2)}, \quad i=1,2, \ldots$, como solução da equação (2.16), e desenvolvendo de forma similar ao procedimento anterior (de (2.17) a (2.19)), definese:

$$
\mathrm{Q}_{\mathrm{i}}^{(2)}=\frac{\mathrm{a}_{4}}{\mathrm{a}_{3}}=-\frac{\operatorname{sen}\left(\left(\lambda_{\mathrm{i}}^{(2)}-1\right) \frac{\alpha}{2}\right)}{\operatorname{sen}\left(\left(\lambda_{\mathrm{i}}^{(2)}+1\right) \frac{\alpha}{2}\right)}=-\frac{\lambda_{\mathrm{i}}^{(2)}-1}{\lambda_{\mathrm{i}}^{(2)}+1} \frac{\cos \left(\left(\lambda_{\mathrm{i}}^{(2)}-1\right) \frac{\alpha}{2}\right)}{\cos \left(\left(\lambda_{\mathrm{i}}^{(2)}+1\right) \frac{\alpha}{2}\right)}
$$

Assim, obtém-se a seguinte função de tensão:

$$
\Phi=\mathrm{r}^{\lambda_{i}^{(2)}+1}\left[\operatorname{sen}\left(\left(\lambda_{i}^{(2)}-1\right) \theta\right)+Q_{i}^{(2)} \operatorname{sen}\left(\left(\lambda_{i}^{(2)}+1\right) \theta\right)\right] .
$$

Nota-se que (2.20) é simétrica em relação a $\theta$, enquanto (2.22) é anti-simétrica. Os campos de tensão e de deslocamento correspondentes a (2.20) são denominados de Modo I e os campos de tensão e deslocamento relacionados a (2.22) são denominados de Modo II.

Para o Modo I, as equações que descrevem as componentes do campo de deslocamento podem ser escritas na forma:

$$
\begin{gathered}
\mathrm{u}_{\mathrm{xi}}^{(1)}=\frac{1}{2 \mathrm{G}} \mathrm{r}^{\lambda_{\mathrm{i}}^{(1)}}\left[\left(\kappa-\mathrm{Q}_{\mathrm{i}}^{(1)}\left(\lambda_{\mathrm{i}}^{(1)}+1\right)\right) \cos \left(\lambda_{\mathrm{i}}^{(1)} \theta\right)-\lambda_{\mathrm{i}}^{(1)} \cos \left(\left(\lambda_{\mathrm{i}}^{(1)}-2\right) \theta\right)\right], \\
\mathrm{u}_{\mathrm{yi}}^{(1)}=\frac{1}{2 \mathrm{G}} \mathrm{r}^{\lambda_{\mathrm{i}}^{(1)}}\left[\left(\kappa+\mathrm{Q}_{\mathrm{i}}^{(1)}\left(\lambda_{\mathrm{i}}^{(1)}+1\right)\right) \operatorname{sen}\left(\lambda_{\mathrm{i}}^{(1)} \theta\right)+\lambda_{\mathrm{i}}^{(1)} \operatorname{sen}\left(\left(\lambda_{\mathrm{i}}^{(1)}-2\right) \theta\right)\right],
\end{gathered}
$$

ou ainda:

$$
\overrightarrow{\mathrm{u}}_{\mathrm{i}}^{(1)}=\left\{\begin{array}{l}
\mathrm{u}_{\mathrm{xi}}^{(1)} \\
\mathrm{u}_{\mathrm{yi}}^{(1)}
\end{array}\right\}=\frac{1}{2 \mathrm{G}} \mathrm{r}^{\lambda_{\mathrm{i}}^{(1)}}\left\{\Psi_{\mathrm{i}}^{(1)}(\theta)\right\}
$$

sendo

$$
\left\{\Psi_{i}^{(1)}(\theta)\right\}=\left\{\begin{array}{l}
\left(\kappa-Q_{i}^{(1)}\left(\lambda_{i}^{(1)}+1\right)\right) \cos \left(\lambda_{i}^{(1)} \theta\right)-\lambda_{i}^{(1)} \cos \left(\left(\lambda_{i}^{(1)}-2\right) \theta\right) \\
\left(\kappa+Q_{i}^{(1)}\left(\lambda_{i}^{(1)}+1\right)\right) \operatorname{sen}\left(\lambda_{i}^{(1)} \theta\right)+\lambda_{i}^{(1)} \operatorname{sen}\left(\left(\lambda_{i}^{(1)}-2\right) \theta\right)
\end{array}\right\}
$$


$\mathrm{G}$ é o módulo de rigidez ao cisalhamento e $\kappa$ é a constante de Kolosov. $\kappa$ é função do coeficiente de Poisson. Para o estado plano de deformação (EPD), $\kappa$ é definida como:

$$
\kappa=3-4 v,
$$

para estado plano de tensão (EPT), tem-se:

$$
\kappa=\frac{3-v}{1+v}
$$

As componentes de tensão para o Modo I são:

$$
\begin{gathered}
\sigma_{\mathrm{xi}}^{(1)}=\lambda_{\mathrm{i}}^{(1)} \mathrm{r}^{\lambda_{\mathrm{i}}^{(1)}-1}\left[\left(2-\mathrm{Q}_{\mathrm{i}}^{(1)}\left(\lambda_{\mathrm{i}}^{(1)}+1\right)\right) \cos \left(\left(\lambda_{\mathrm{i}}^{(1)}-1\right) \theta\right)-\left(\lambda_{\mathrm{i}}^{(1)}-1\right) \cos \left(\left(\lambda_{\mathrm{i}}^{(1)}-3\right) \theta\right)\right] \\
\sigma_{\mathrm{yi}}^{(1)}=\lambda_{\mathrm{i}}^{(1)} \mathrm{r}^{\lambda_{\mathrm{i}}^{(1)}-1}\left[\left(2+\mathrm{Q}_{\mathrm{i}}^{(1)}\left(\lambda_{\mathrm{i}}^{(1)}+1\right)\right) \cos \left(\left(\lambda_{\mathrm{i}}^{(1)}-1\right) \theta\right)+\left(\lambda_{\mathrm{i}}^{(1)}-1\right) \cos \left(\left(\lambda_{\mathrm{i}}^{(1)}-3\right) \theta\right)\right] \\
\tau_{\mathrm{xyi}}^{(1)}=\lambda_{\mathrm{i}}^{(1)} \mathrm{r}_{\mathrm{i}}^{\lambda_{1}^{(1)}-1}\left[\left(\lambda_{\mathrm{i}}^{(1)}-1\right) \operatorname{sen}\left(\left(\lambda_{\mathrm{i}}^{(1)}-3\right) \theta\right)+\mathrm{Q}_{\mathrm{i}}^{(1)}\left(\lambda_{\mathrm{i}}^{(1)}+1\right) \operatorname{sen}\left(\left(\lambda_{\mathrm{i}}^{(1)}-1\right) \theta\right)\right]
\end{gathered}
$$

Para o Modo II, as equações que descrevem as componentes do campo de deslocamento podem ser escritas na forma:

$$
\begin{gathered}
\mathrm{u}_{\mathrm{xi}}^{(2)}=\frac{1}{2 \mathrm{G}} \mathrm{r}^{\lambda_{i}^{(2)}}\left[\left(\kappa-\mathrm{Q}_{\mathrm{i}}^{(2)}\left(\lambda_{\mathrm{i}}^{(2)}+1\right)\right) \operatorname{sen}\left(\lambda_{\mathrm{i}}^{(2)} \theta\right)-\lambda_{\mathrm{i}}^{(2)} \operatorname{sen}\left(\left(\lambda_{\mathrm{i}}^{(2)}-2\right) \theta\right)\right], \\
\mathrm{u}_{\mathrm{yi}}^{(2)}=-\frac{1}{2 \mathrm{G}} \mathrm{r}^{\lambda_{\mathrm{i}}^{(2)}}\left[\left(\kappa+\mathrm{Q}_{\mathrm{i}}^{(2)}\left(\lambda_{\mathrm{i}}^{(2)}+1\right)\right) \cos \left(\lambda_{\mathrm{i}}^{(2)} \theta\right)+\lambda_{\mathrm{i}}^{(2)} \cos \left(\left(\lambda_{\mathrm{i}}^{(2)}-2\right) \theta\right)\right],
\end{gathered}
$$

ou ainda:

$$
\overrightarrow{\mathrm{u}}_{\mathrm{i}}^{(2)}=\left\{\begin{array}{l}
\mathrm{u}_{\mathrm{xi}}^{(2)} \\
\mathrm{u}_{\mathrm{yi}}^{(2)}
\end{array}\right\}=\frac{1}{2 \mathrm{G}} \mathrm{r}^{\lambda_{\mathrm{i}}^{(2)}}\left\{\Psi_{\mathrm{i}}^{(2)}(\theta)\right\},
$$


sendo

$$
\left\{\Psi_{i}^{(2)}(\theta)\right\}=\left\{\begin{array}{c}
\left(\kappa-Q_{i}^{(2)}\left(\lambda_{i}^{(2)}+1\right)\right) \operatorname{sen}\left(\lambda_{i}^{(2)} \theta\right)-\lambda_{i}^{(2)} \operatorname{sen}\left(\left(\lambda_{i}^{(2)}-2\right) \theta\right) \\
-\left(\kappa+Q_{i}^{(2)}\left(\lambda_{i}^{(2)}+1\right)\right) \cos \left(\lambda_{i}^{(2)} \theta\right)-\lambda_{i}^{(2)} \cos \left(\left(\lambda_{i}^{(2)}-2\right) \theta\right)
\end{array}\right\}
$$

As componentes de tensão para o Modo II são:

$$
\begin{gathered}
\sigma_{\mathrm{xi}}^{(2)}=\lambda_{\mathrm{i}}^{(2)} \mathrm{r}_{\mathrm{i}}^{\lambda_{i}^{(2)}-1}\left[\left(2-\mathrm{Q}_{\mathrm{i}}^{(2)}\left(\lambda_{\mathrm{i}}^{(2)}+1\right)\right) \operatorname{sen}\left(\left(\lambda_{\mathrm{i}}^{(2)}-1\right) \theta\right)-\left(\lambda_{\mathrm{i}}^{(2)}-1\right) \operatorname{sen}\left(\left(\lambda_{\mathrm{i}}^{(2)}-3\right) \theta\right)\right] \\
\sigma_{\mathrm{yi}}^{(2)}=\lambda_{\mathrm{i}}^{(2)} \mathrm{r}_{\mathrm{i}}^{\lambda_{i}^{(2)}-1}\left[\left(2+\mathrm{Q}_{\mathrm{i}}^{(2)}\left(\lambda_{\mathrm{i}}^{(2)}+1\right)\right) \operatorname{sen}\left(\left(\lambda_{\mathrm{i}}^{(2)}-1\right) \theta\right)+\left(\lambda_{\mathrm{i}}^{(2)}-1\right) \operatorname{sen}\left(\left(\lambda_{\mathrm{i}}^{(2)}-3\right) \theta\right)\right] \\
\tau_{\mathrm{xyi}}^{(2)}=-\lambda_{\mathrm{i}}^{(2)} \mathrm{r}^{\lambda_{\mathrm{i}}^{(2)}-1}\left[\left(\lambda_{\mathrm{i}}^{(2)}-1\right) \cos \left(\left(\lambda_{\mathrm{i}}^{(2)}-3\right) \theta\right)+\mathrm{Q}_{\mathrm{i}}^{(2)}\left(\lambda_{\mathrm{i}}^{(2)}+1\right) \cos \left(\left(\lambda_{\mathrm{i}}^{(2)}-1\right) \theta\right)\right] .
\end{gathered}
$$

O desenvolvimento apresentado é válido para uma região reentrante no domínio de ângulo $\alpha$ qualquer (Figura 2.5). No caso da mecânica da fratura, particulariza-se a solução para $\alpha=2 \pi$. Neste caso, as equações (2.15) e (2.16) são idênticas e suas raízes são reais e simples (não repetidas ou multiplicidade 1).

$$
\begin{aligned}
& \operatorname{sen}(\lambda 2 \pi)=0 \\
& \lambda= \pm \frac{\mathrm{i}}{2}
\end{aligned}
$$

sendo $\mathrm{i} \neq 0$ e $\mathrm{i} \neq 2$, pois $\lambda \neq 0$ e $\lambda \neq \pm 1$, assim:

$$
\lambda_{i}^{(1)}=\lambda_{i}^{(2)}= \pm \frac{1}{2}, \pm \frac{3}{2}, \pm 2, \pm \frac{5}{2} \cdots
$$

Assumindo que as faces da fissura possuem tensões nulas, energia de deformação finita e desprezando os deslocamentos e rotações de corpo rígido, qualquer solução na vizinhança da fissura pode ser escrita na forma:

$$
\left\{\begin{array}{l}
\mathrm{u}_{\mathrm{x}} \\
\mathrm{u}_{\mathrm{y}}
\end{array}\right\}=\sum_{\mathrm{i}=1}^{\infty} \frac{\mathrm{A}_{\mathrm{i}}^{(1)}}{2 \mathrm{G}} \mathrm{r}^{\lambda_{\mathrm{i}}^{(1)}}\left\{\Psi_{\mathrm{i}}^{(1)}(\theta)\right\}+\sum_{\mathrm{i}=1}^{\infty} \frac{\mathrm{A}_{\mathrm{i}}^{(2)}}{2 \mathrm{G}} \mathrm{r}^{\lambda_{\mathrm{i}}^{(2)}}\left\{\Psi_{\mathrm{i}}^{(2)}(\theta)\right\} .
$$


A série infinita da equação (2.41) converge absolutamente para $r<r_{o}$ para qualquer $r_{o}>0$. Os coeficientes $A_{1}^{(1)}$ e $A_{1}^{(2)}$ são chamados fatores de intensidade de tensão generalizados. A relação entre os coeficientes $A_{1}^{(1)}$ e $A_{1}^{(2)}$ e os fatores de intensidade de tensão do Modo I e do Modo II é da forma:

$$
\mathrm{A}_{1}^{(1)}=\frac{\mathrm{K}_{\mathrm{I}}}{\sqrt{2 \pi}}, \quad \mathrm{A}_{1}^{(2)}=\frac{\mathrm{K}_{\mathrm{II}}}{\sqrt{2 \pi}}
$$

Do ponto de vista de análise de engenharia, os vértices com ângulo $\alpha$ de $360^{\circ}$, $270^{\circ}, 240^{\circ}, 225^{\circ}$ e $210^{\circ}$ são de grande importância (SZABÓ; BABUŠKA, 1991). Para estes ângulos os valores de $\lambda_{1}$ para os Modos I e II são:

Tabela 2.1 - Valores de $\lambda_{1}$ para os Modos de deformação I e II para vértice com ângulo $\alpha$ (SZABÓ; BABUŠKA, 1991).

\begin{tabular}{ccc}
\hline$\alpha$ & $\lambda_{1}^{(1)}$ & $\lambda_{1}^{(2)}$ \\
\hline $360^{\circ}$ & 0,500000 & 0,500000 \\
$270^{\circ}$ & 0,544484 & 0,908529 \\
$240^{\circ}$ & 0,615731 & 1,148913 \\
$225^{\circ}$ & 0,673583 & 1,302086 \\
$210^{\circ}$ & 0,751975 & 1,485812 \\
\hline
\end{tabular}

As equações da elasticidade para a vizinhança em torno de uma região reentrante no domínio, desenvolvidas neste capítulo, serão utilizadas na formulação dos métodos de extração dos coeficientes $\mathrm{A}_{\mathrm{i}}^{(\mathrm{n})}$ da expansão assintótica e, em particular, para extração dos fatores de intensidade de tensão. No Capítulo 3 serão apresentadas as formulações dos métodos de extração utilizados no presente trabalho. 


\section{Formulação dos métodos de extração}

A extração dos fatores de intensidade de tensão é de grande importância para a análise do comportamento da fissura em um sólido. Neste capítulo são demonstradas as formulações dos métodos da integral de contorno (MIC) e da função cutoff (MFC). Esses métodos foram desenvolvidos por Szabó e Babuška (1988) no contexto do método de elementos finitos (MEF). As formulações desenvolvidas por Szabó e Babuška (1988), para os métodos de extração, servem não só para a extração dos fatores de intensidade de tensão, mas também para a extração de outros coeficientes da expansão assintótica. No presente trabalho, tais métodos são utilizados para extrair os fatores de intensidade de tensão da solução do método de elementos finitos generalizados (MEFG).

\subsection{Integral independente do caminho - Lei de Betti}

Seja $\overrightarrow{\mathrm{u}}$ um campo de deslocamento para o problema elástico plano, sendo:

$$
\overrightarrow{\mathrm{u}}=\left(\begin{array}{l}
\mathrm{u}_{\mathrm{x}}(\mathrm{x}, \mathrm{y}) \\
\mathrm{u}_{\mathrm{y}}(\mathrm{x}, \mathrm{y})
\end{array}\right) .
$$

A representação vetorial do tensor de deformação, correspondente ao campo de deslocamento (3.1), e suas respectivas componentes são definidos da seguinte forma:

$$
\left\{\varepsilon^{(\mathrm{u})}\right\}=\left\{\varepsilon_{\mathrm{x}}^{(\mathrm{u})} \quad \varepsilon_{\mathrm{y}}^{(\mathrm{u})} \quad \gamma_{\mathrm{xy}}^{(\mathrm{u})}\right\}^{\mathrm{T}}
$$


As relações entre deformação e deslocamento são:

$$
\begin{aligned}
& \varepsilon_{\mathrm{x}}^{(\mathrm{u})}=\frac{\partial \mathrm{u}_{\mathrm{x}}}{\partial \mathrm{x}} \\
& \varepsilon_{\mathrm{y}}^{(\mathrm{u})}=\frac{\partial \mathrm{u}_{\mathrm{y}}}{\partial \mathrm{y}} \\
& \gamma_{\mathrm{xy}}^{(\mathrm{u})}=\frac{\partial \mathrm{u}_{\mathrm{x}}}{\partial \mathrm{y}}+\frac{\partial \mathrm{u}_{\mathrm{y}}}{\partial \mathrm{x}}
\end{aligned}
$$

Similarmente, definem-se a representação vetorial do tensor de tensão e suas componentes correspondentes ao campo de deslocamentos (3.1):

$$
\left\{\sigma^{(\mathrm{u})}\right\}=\left\{\sigma_{\mathrm{x}}^{(\mathrm{u})} \quad \sigma_{\mathrm{y}}^{(\mathrm{u})} \quad \tau_{\mathrm{xy}}^{(\mathrm{u})}\right\}^{\mathrm{T}} .
$$

A relação entre tensão e deformação é:

$$
\left\{\sigma^{(\mathrm{u})}\right\}=[\mathrm{E}]\left\{\varepsilon^{(\mathrm{u})}\right\}
$$

sendo [E] uma matriz simétrica, positiva definida, denominada de matriz constitutiva de rigidez do material. Considerando-se forças de volume nulas, deformações iniciais nulas e que o campo de deslocamentos (3.1), bem como o tensor de tensão correspondente (3.5), satisfazem as equações de equilíbrio, tem-se:

$$
\begin{aligned}
& \frac{\partial \sigma_{\mathrm{x}}^{(\mathrm{u})}}{\partial \mathrm{x}}+\frac{\partial \tau_{\mathrm{xy}}^{(\mathrm{u})}}{\partial \mathrm{y}}=0 \\
& \frac{\partial \tau_{\mathrm{xy}}^{(\mathrm{u})}}{\partial \mathrm{x}}+\frac{\partial \sigma_{\mathrm{y}}^{(\mathrm{u})}}{\partial \mathrm{y}}=0
\end{aligned}
$$

Sejam $\mathrm{n}_{\mathrm{x}}$ e $\mathrm{n}_{\mathrm{y}}$ os co-senos diretores de um vetor normal ao contorno do domínio de um corpo elástico plano, em um ponto $\mathrm{P}$ qualquer pertencente ao contorno, a relação entre as componentes do vetor tração e o tensor de tensão no ponto $\mathrm{P}$ é:

$$
\begin{aligned}
& \mathrm{T}_{\mathrm{x}}^{(\mathrm{u})}=\sigma_{\mathrm{x}}^{(\mathrm{u})} \mathrm{n}_{\mathrm{x}}+\tau_{\mathrm{xy}}^{(\mathrm{u})} \mathrm{n}_{\mathrm{y}} . \\
& \mathrm{T}_{\mathrm{y}}^{(\mathrm{u})}=\tau_{\mathrm{xy}}^{(\mathrm{u})} \mathrm{n}_{\mathrm{x}}+\sigma_{\mathrm{y}}^{(\mathrm{u})} \mathrm{n}_{\mathrm{y}} .
\end{aligned}
$$


Seja $\overrightarrow{\mathrm{V}}$ (3.8) um campo de deslocamento arbitrário e que a energia de deformação associada a este seja finita no domínio $\Omega$ (Figura 3.1). Admite-se que as trações (3.7) são determinadas ao longo do contorno $\partial \Omega$ (Figura 3.1).

$$
\vec{v}=\left(\begin{array}{c}
v_{x}(x, y) \\
v_{y}(x, y)
\end{array}\right)
$$

$\mathrm{Na}$ ausência de forças de volume, ações térmicas e restrições elásticas, o princípio dos trabalhos virtuais (PTV) pode ser escrito na forma:

$$
\underbrace{\iint_{\Omega}\left\{\varepsilon^{(\mathrm{v})}\right\}^{\mathrm{T}}\left\{\sigma^{(\mathrm{u})}\right\} \mathrm{dxdy}}_{\text {Trabalho Vitual das Forças Internas }}=\underbrace{\int_{\partial \Omega}\left(\mathrm{T}_{\mathrm{x}}^{(\mathrm{u})} \mathrm{v}_{\mathrm{x}}+\mathrm{T}_{\mathrm{y}}^{(\mathrm{u})} \mathrm{v}_{\mathrm{y}}\right) \mathrm{ds}}_{\text {Trabalho Virtual das Forças Externas }}
$$

para todo deslocamento virtual $\overrightarrow{\mathrm{V}}(3.8)$.

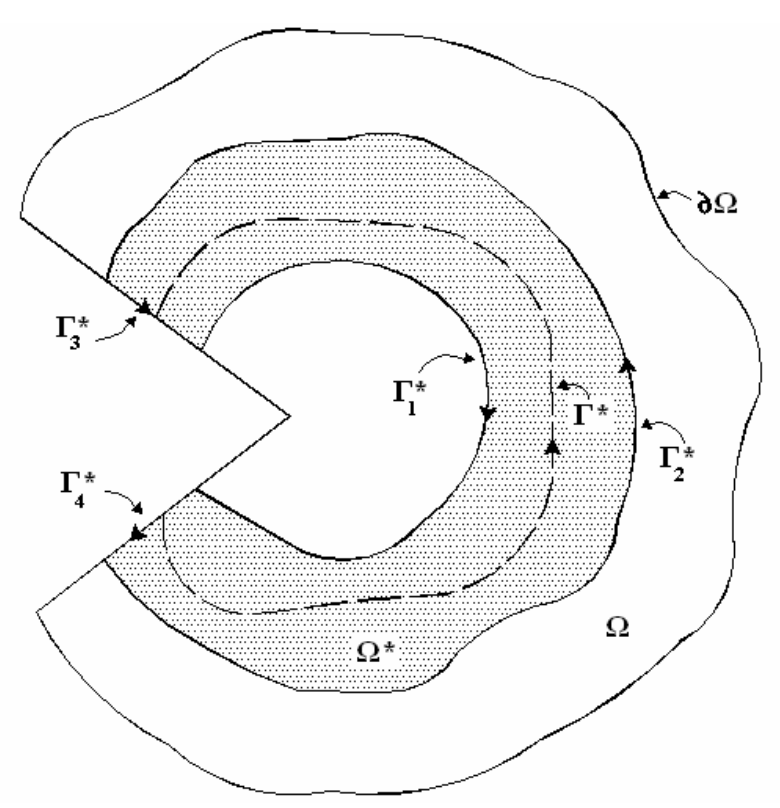

Figura 3.1 - Domínio do problema $\Omega$ e subdomínio de integração $\Omega^{*}$.

As componentes dos tensores de deformação e de tensão associados a $\vec{v}$ são definidas por meio das equações (3.2), (3.3), (3.4) e (3.5). Pela simetria de [E] e utilizando as equações (3.5) e (3.9), tem-se: 


$$
\begin{aligned}
\iint_{\Omega}\left\{\varepsilon^{(\mathrm{v})}\right\}^{\mathrm{T}}[\mathrm{E}]\left\{\varepsilon^{(\mathrm{u})}\right\} \mathrm{dxdy} & \equiv \iint_{\Omega}\left([\mathrm{E}]\left\{\varepsilon^{(\mathrm{v})}\right\}\right)^{\mathrm{T}}\left\{\varepsilon^{(\mathrm{u})}\right\} \mathrm{dxdy} \\
& \equiv \iint_{\Omega}\left\{\sigma^{(\mathrm{v})}\right\}^{\mathrm{T}}\left\{\varepsilon^{(\mathrm{u})}\right\} \mathrm{dxdy}=\int_{\partial \Omega}\left(\mathrm{T}_{\mathrm{x}}^{(\mathrm{u})} \mathrm{v}_{\mathrm{x}}+\mathrm{T}_{\mathrm{y}}^{(\mathrm{u})} \mathrm{v}_{\mathrm{y}}\right) \mathrm{ds}
\end{aligned}
$$

A equação (3.10) ainda pode ser escrita na seguinte forma:

$$
\iint_{\Omega}\left[\sigma_{\mathrm{x}}^{(\mathrm{v})} \frac{\partial \mathrm{u}_{\mathrm{x}}}{\partial \mathrm{x}}+\sigma_{\mathrm{y}}^{(\mathrm{v})} \frac{\partial \mathrm{u}_{\mathrm{y}}}{\partial \mathrm{y}}+\tau_{\mathrm{xy}}^{(\mathrm{v})}\left(\frac{\partial \mathrm{u}_{\mathrm{x}}}{\partial \mathrm{y}}+\frac{\partial \mathrm{u}_{\mathrm{y}}}{\partial \mathrm{x}}\right)\right] \mathrm{dxdy}=\int_{\partial \Omega}\left(\mathrm{T}_{\mathrm{x}}^{(\mathrm{u})} \mathrm{v}_{\mathrm{x}}+\mathrm{T}_{\mathrm{y}}^{(\mathrm{u})} \mathrm{v}_{\mathrm{y}}\right) \mathrm{ds}
$$

Aplicando-se o teorema de Green, tem-se:

$$
\begin{aligned}
\int_{\partial \Omega}\left(\mathrm{T}_{\mathrm{x}}^{(\mathrm{v})} \mathrm{u}_{\mathrm{x}}+\mathrm{T}_{\mathrm{y}}^{(\mathrm{v})} \mathrm{u}_{\mathrm{y}}\right) \mathrm{ds} & -\int_{\partial \Omega}\left(\mathrm{T}_{\mathrm{x}}^{(\mathrm{u})} \mathrm{v}_{\mathrm{x}}+\mathrm{T}_{\mathrm{y}}^{(\mathrm{u})} \mathrm{v}_{\mathrm{y}}\right) \mathrm{ds} \\
& =\iint_{\Omega}\left[\left(\frac{\partial \sigma_{x}^{(\mathrm{v})}}{\partial \mathrm{x}}+\frac{\partial \tau_{\mathrm{xy}}^{(\mathrm{v})}}{\partial \mathrm{y}}\right) \mathrm{u}_{\mathrm{x}}+\left(\frac{\partial \tau_{\mathrm{xy}}^{(\mathrm{v})}}{\partial \mathrm{x}}+\frac{\partial \sigma_{\mathrm{y}}^{(\mathrm{v})}}{\partial \mathrm{y}}\right) \mathrm{u}_{\mathrm{y}}\right] \mathrm{dxdy}
\end{aligned}
$$

Considerando-se que as tensões correspondentes aos campos $\vec{u}$ (3.1) e $\vec{v}$ (3.8) satisfazem as equações de equilíbrio (3.6) e que as forças de volume são nulas, chega-se a Lei de Betti

$$
\int_{\partial \Omega}\left(\mathrm{T}_{\mathrm{x}}^{(\mathrm{v})} \mathrm{u}_{\mathrm{x}}+\mathrm{T}_{\mathrm{y}}^{(\mathrm{v})} \mathrm{u}_{\mathrm{y}}\right) \mathrm{ds}=\int_{\partial \Omega}\left(\mathrm{T}_{\mathrm{x}}^{(\mathrm{u})} \mathrm{v}_{\mathrm{x}}+\mathrm{T}_{\mathrm{y}}^{(\mathrm{u})} \mathrm{v}_{\mathrm{y}}\right) \mathrm{ds}
$$

em (3.13) a integração é feita em torno do domínio $\Omega$ no sentido anti-horário (Figura $3.1)$.

Adota-se um subdomínio de $\Omega$, denominado de $\Omega^{*}$, na vizinhança da região reentrante do domínio (Figura 3.1). $\Omega^{*}$ é contornado por duas curvas contínuas $\left(\Gamma_{1}^{*} \mathrm{e}\right.$ $\left.\Gamma_{2}^{*}\right)$ e por trechos das arestas $\left(\Gamma_{3}^{*}\right.$ e $\left.\Gamma_{4}^{*}\right)$. Sendo assim, cada integral da equação (3.13) pode ser subdividida da seguinte forma:

$$
\begin{aligned}
\int_{\partial \Omega^{*}}\left(\mathrm{~T}_{\mathrm{x}}^{(\mathrm{u})} \mathrm{v}_{\mathrm{x}}+\mathrm{T}_{\mathrm{y}}^{(\mathrm{u})} \mathrm{v}_{\mathrm{y}}\right) \mathrm{ds} & =\int_{\Gamma_{1}^{*}}\left(\mathrm{~T}_{\mathrm{x}}^{(\mathrm{u})} \mathrm{v}_{\mathrm{x}}+\mathrm{T}_{\mathrm{y}}^{(\mathrm{u})} \mathrm{v}_{\mathrm{y}}\right) \mathrm{ds}+\int_{\Gamma_{2}^{*}}\left(\mathrm{~T}_{\mathrm{x}}^{(\mathrm{u})} \mathrm{v}_{\mathrm{x}}+\mathrm{T}_{\mathrm{y}}^{(\mathrm{u})} \mathrm{v}_{\mathrm{y}}\right) \mathrm{ds} \\
& +\int_{\Gamma_{3}^{*}}\left(\mathrm{~T}_{\mathrm{x}}^{(\mathrm{u})} \mathrm{v}_{\mathrm{x}}+\mathrm{T}_{\mathrm{y}}^{(\mathrm{u})} \mathrm{v}_{\mathrm{y}}\right) \mathrm{ds}+\int_{\Gamma_{4}^{*}}\left(\mathrm{~T}_{\mathrm{x}}^{(\mathrm{u})} \mathrm{v}_{\mathrm{x}}+\mathrm{T}_{\mathrm{y}}^{(\mathrm{u})} \mathrm{v}_{\mathrm{y}}\right) \mathrm{ds}
\end{aligned}
$$


Admite-se que os campos $\overrightarrow{\mathrm{u}}$ (3.1) e $\overrightarrow{\mathrm{v}}$ (3.8) satisfazem as condições de contorno de tensão nula nas arestas $\left(\sigma_{\theta}=\tau_{\mathrm{r} \theta}=0\right.$ em $\left.\theta= \pm \alpha / 2\right)$. Assim, utilizando-se as equações (3.13) e (3.14), tem-se:

$$
\begin{aligned}
\int_{\Gamma_{1}^{*}}\left(\mathrm{~T}_{\mathrm{x}}^{(\mathrm{v})} \mathrm{u}_{\mathrm{x}}+\mathrm{T}_{\mathrm{y}}^{(\mathrm{v})} \mathrm{u}_{\mathrm{y}}\right) \mathrm{ds} & +\int_{\Gamma_{2}^{*}}\left(\mathrm{~T}_{\mathrm{x}}^{(\mathrm{v})} \mathrm{u}_{\mathrm{x}}+\mathrm{T}_{\mathrm{y}}^{(\mathrm{v})} \mathrm{u}_{\mathrm{y}}\right) \mathrm{ds} \\
& =\int_{\Gamma_{1}^{*}}\left(\mathrm{~T}_{\mathrm{x}}^{(\mathrm{u})} \mathrm{v}_{\mathrm{x}}+\mathrm{T}_{\mathrm{y}}^{(\mathrm{u})} \mathrm{v}_{\mathrm{y}}\right) \mathrm{ds}+\int_{\Gamma_{2}^{*}}\left(\mathrm{~T}_{\mathrm{x}}^{(\mathrm{u})} \mathrm{v}_{\mathrm{x}}+\mathrm{T}_{\mathrm{y}}^{(\mathrm{u})} \mathrm{v}_{\mathrm{y}}\right) \mathrm{ds}
\end{aligned}
$$

em (3.15) a integral é feita em torno do domínio $\Omega^{*}$ no sentido anti-horário.

Na equação (3.15), a integração ao longo do contorno $\Gamma_{1}^{*}$ é feita no sentido horário com relação à região reentrante no domínio (Figura 3.1), enquanto a integral em $\Gamma_{2}^{*}$ é feita no sentido anti-horário. Invertendo-se o sentido de integração ao longo do contorno $\Gamma_{1}^{*}$, as integrais ao longo dos contornos $\Gamma_{1}^{*}$ e $\Gamma_{2}^{*}$ são determinadas no sentido anti-horário com relação a região reentrante do domínio.

$$
\begin{aligned}
\int_{\tilde{\Gamma}_{1}^{*}}\left(\mathrm{~T}_{\mathrm{x}}^{(\mathrm{u})} \mathrm{v}_{\mathrm{x}}+\mathrm{T}_{\mathrm{y}}^{(\mathrm{u})} \mathrm{v}_{\mathrm{y}}\right) \mathrm{ds} & -\int_{\tilde{\Gamma}_{1}^{*}}\left(\mathrm{~T}_{\mathrm{x}}^{(\mathrm{v})} \mathrm{u}_{\mathrm{x}}+\mathrm{T}_{\mathrm{y}}^{(\mathrm{v})} \mathrm{u}_{\mathrm{y}}\right) \mathrm{ds} \\
& =\int_{\Gamma_{2}^{*}}\left(\mathrm{~T}_{\mathrm{x}}^{(\mathrm{u})} \mathrm{v}_{\mathrm{x}}+\mathrm{T}_{\mathrm{y}}^{(\mathrm{u})} \mathrm{v}_{\mathrm{y}}\right) \mathrm{ds}-\int_{\Gamma_{2}^{*}}\left(\mathrm{~T}_{\mathrm{x}}^{(\mathrm{v})} \mathrm{u}_{\mathrm{x}}+\mathrm{T}_{\mathrm{y}}^{(\mathrm{v})} \mathrm{u}_{\mathrm{y}}\right) \mathrm{ds}
\end{aligned}
$$

Na equação (3.16), $\widetilde{\Gamma}_{1}^{*}$ indica a integração ao longo do contorno $\Gamma_{1}^{*}$ no sentido anti-horário. Como $\widetilde{\Gamma}_{1}^{*}$ e $\Gamma_{2}^{*}$ são arbitrários, conclui-se que a integral (3.17) é independente do caminho. O caminho $\Gamma^{*}$ (3.17) deve começar em uma aresta da região reentrante e terminar na outra sempre no sentido anti-horário, como apresentado na Figura 3.1.

$$
\mathrm{I}_{\Gamma^{*}}(\overrightarrow{\mathrm{u}}, \overrightarrow{\mathrm{v}})=\int_{\Gamma^{*}}\left(\mathrm{~T}_{\mathrm{x}}^{(\mathrm{u})} \mathrm{v}_{\mathrm{x}}+\mathrm{T}_{\mathrm{y}}^{(\mathrm{u})} \mathrm{v}_{\mathrm{y}}\right) \mathrm{ds}-\int_{\Gamma^{*}}\left(\mathrm{~T}_{\mathrm{x}}^{(\mathrm{v})} \mathrm{u}_{\mathrm{x}}+\mathrm{T}_{\mathrm{y}}^{(\mathrm{v})} \mathrm{u}_{\mathrm{y}}\right) \mathrm{ds}
$$

Quando o tensor de tensão correspondente ao campo $\overrightarrow{\mathrm{V}}$ (3.8) não satisfaz as equações de equilíbrio e nem as condições de contorno em tensão nas arestas da região reentrante, substitui-se (3.17) por: 


$$
\begin{aligned}
& \int_{\tilde{\Gamma}_{1}^{*}}\left(\mathrm{~T}_{\mathrm{x}}^{(\mathrm{u})} \mathrm{v}_{\mathrm{x}}+\mathrm{T}_{\mathrm{y}}^{(\mathrm{u})} \mathrm{v}_{\mathrm{y}}\right) \mathrm{ds}-\int_{\tilde{\Gamma}_{1}^{*}}\left(\mathrm{~T}_{\mathrm{x}}^{(\mathrm{v})} \mathrm{u}_{\mathrm{x}}+\mathrm{T}_{\mathrm{y}}^{(\mathrm{v})} \mathrm{u}_{\mathrm{y}}\right) \mathrm{ds} \\
& \quad=\int_{\Gamma_{2}^{*}}\left(\mathrm{~T}_{\mathrm{x}}^{(\mathrm{u})} \mathrm{v}_{\mathrm{x}}+\mathrm{T}_{\mathrm{y}}^{(\mathrm{u})} \mathrm{v}_{\mathrm{y}}\right) \mathrm{ds}-\int_{\Gamma_{2}^{*}}\left(\mathrm{~T}_{\mathrm{x}}^{(\mathrm{v})} \mathrm{u}_{\mathrm{x}}+\mathrm{T}_{\mathrm{y}}^{(\mathrm{v})} \mathrm{u}_{\mathrm{y}}\right) \mathrm{ds} \\
&-\int_{\Gamma_{3}^{*}}\left(\mathrm{~T}_{\mathrm{x}}^{(\mathrm{v})} \mathrm{u}_{\mathrm{x}}+\mathrm{T}_{\mathrm{y}}^{(\mathrm{v})} \mathrm{u}_{\mathrm{y}}\right) \mathrm{ds}-\int_{\Gamma_{4}^{*}}\left(\mathrm{~T}_{\mathrm{x}}^{(\mathrm{v})} \mathrm{u}_{\mathrm{x}}+\mathrm{T}_{\mathrm{y}}^{(\mathrm{v})} \mathrm{u}_{\mathrm{y}}\right) \mathrm{ds} \\
&+\iint_{\Omega^{*}}\left[\left(\frac{\partial \sigma_{\mathrm{x}}^{(\mathrm{v})}}{\partial \mathrm{x}}+\frac{\partial \mathrm{r}_{\mathrm{xy}}^{(\mathrm{v})}}{\partial \mathrm{y}}\right) \mathrm{u}_{\mathrm{x}}+\left(\frac{\partial \tau_{\mathrm{xy}}^{(\mathrm{v})}}{\partial \mathrm{x}}+\frac{\partial \sigma_{\mathrm{y}}^{(\mathrm{v})}}{\partial \mathrm{y}}\right) \mathrm{u}_{\mathrm{y}}\right] \mathrm{dxdy}
\end{aligned}
$$

\subsection{Ortogonalidade das autofunções}

No desenvolvimento da solução da elasticidade na vizinhança de uma região reentrante no domínio (Seção 2.3) foram consideradas apenas raízes reais e simples (não repetidas ou multiplicidade 1). Tal consideração é particularmente importante, pois para a mecânica da fratura elástica linear os autovalores são reais e simples e as autofunções são da forma:

$$
\left\{\phi_{\mathrm{i}}^{(\mathrm{n})}\right\}=\mathrm{r}^{\lambda_{\mathrm{i}}^{(\mathrm{n})}}\left\{\Psi_{\mathrm{i}}^{(\mathrm{n})}\right\}
$$

$\mathrm{n}(\mathrm{n}=1,2)$ representa as partes simétrica e anti-simétrica da solução, respectivamente. $\left\{\Psi_{i}^{(1)}\right\}(2.26)$ e $\left\{\Psi_{i}^{(2)}\right\}(2.35)$, definidas na seção 2.3, são funções ortogonais.

A ortogonalidade entre funções pode ser interpretada como uma generalização da propriedade de ortogonalidade entre dois vetores em um espaço de dimensão i (3.19). As componentes do vetor podem ser representadas por funções contínuas de variáveis independentes e $\mathrm{i}$ indica a quantidade de funções.

Considera-se que os campos de deslocamento $\vec{u}$ (3.1) e $\vec{v}$ (3.8) são funções na forma:

$$
\overrightarrow{\mathrm{u}}_{\mathrm{i}}=\frac{1}{2 \mathrm{G}} \mathrm{r}^{\lambda_{\mathrm{i}}^{(\mathrm{n})}}\left\{\Psi_{\mathrm{i}}^{(\mathrm{n})}\right\}, \quad \overrightarrow{\mathrm{v}}_{-\mathrm{j}}=\frac{1}{2 \mathrm{G}} \mathrm{r}^{\lambda_{-\mathrm{j}}^{(\mathrm{m})}}\left\{\Psi_{-\mathrm{j}}^{(\mathrm{m})}\right\}, \quad \mathrm{n}, \mathrm{m}=1,2
$$

No campo de deslocamento $\overrightarrow{\mathrm{v}}$, o índice $-\mathrm{j}$ serve para indicar o uso de autovalores negativos na determinação deste campo. Sendo assim, $\lambda_{-\mathrm{j}}^{(\mathrm{m})}=-\lambda_{\mathrm{j}}^{(\mathrm{m})}$. 
Assume-se, na integral (3.17), o contorno $\Gamma^{*}=\Gamma_{\rho}$. Sendo $\Gamma_{\rho}$ um arco circular de raio $\rho$, centrado no vértice da quina reentrante, como ilustrado na Figura 3.2.

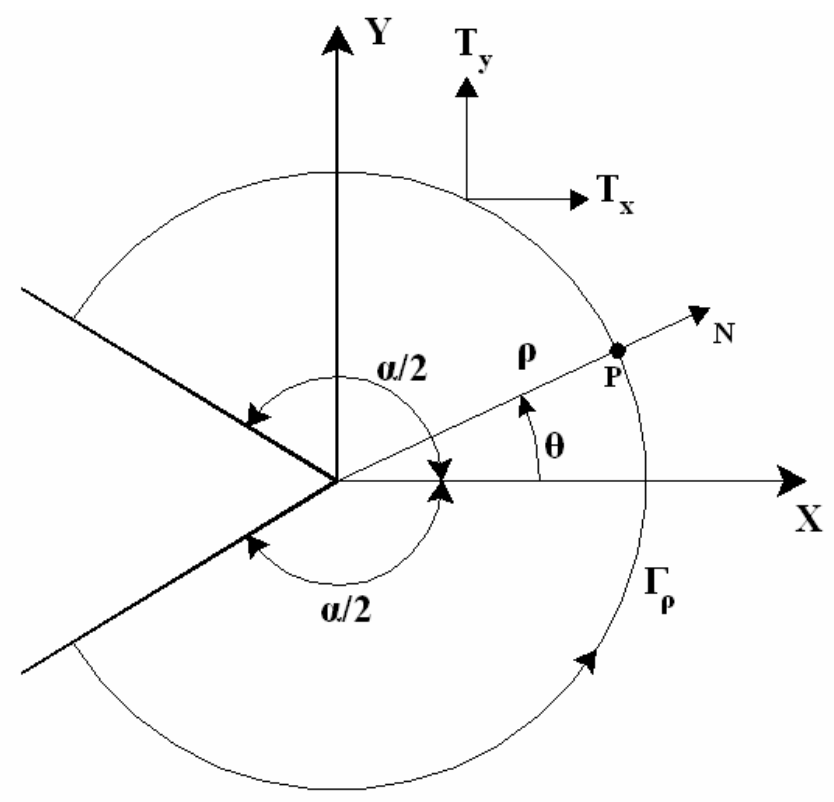

Figura 3.2 - Caminho de integração circular $\left(\Gamma_{\rho}\right)$ na vizinhança da região reentrante.

O vetor tração correspondente ao campo de deslocamento $\overrightarrow{\mathrm{u}}_{\mathrm{i}}$ no contorno $\Gamma_{\rho}$ é escrito na forma:

$$
\left\{\mathrm{T}_{\mathrm{i}}^{\left(\mathrm{u}_{\mathrm{i}}\right)}\right\}=\lambda_{\mathrm{i}}^{(\mathrm{n})} \rho^{\lambda_{\mathrm{i}}^{(\mathrm{n})}-1}\left\{\mathrm{Y}_{\mathrm{i}}^{(\mathrm{n})}\right\}
$$

$\left\{\mathrm{T}_{\mathrm{i}}^{(\mathrm{n})}\right\}$ é calculado por meio da equação (3.22)

$$
\left\{\mathrm{T}_{\mathrm{i}}^{(\mathrm{n})}\right\}=\left[\sigma_{\mathrm{i}}^{(\mathrm{n})}\right]\{\mathrm{N}\} ; \quad \text { onde }\{\mathrm{N}\}=\{\cos (\theta) \quad \operatorname{sen}(\theta)\}^{\mathrm{T}}
$$

sendo $\{\mathrm{N}\}$ um vetor unitário normal ao contorno $\Gamma_{\rho}$ em um ponto $\mathrm{P}$ qualquer (Figura 3.2). As componentes do tensor $\left[\sigma_{i}^{(\mathrm{n})}\right]$ são as equações (2.29), (2.30) e (2.31) para a parte simétrica e as equações (2.36), (2.37) e (2.38) para a parte anti-simétrica. Assim, a função $\left\{Y_{i}^{(n)}\right\}$ pode ser escrita da seguinte forma: para $n=1$ (parte simétrica) 


$$
\left\{Y_{i}^{(1)}\right\}=\left\{\begin{array}{l}
{\left[\left(2-Q_{i}^{(1)}\left(\lambda_{i}^{(1)}+1\right)\right) \cos \left(\left(\lambda_{i}^{(1)}-1\right) \theta\right)-\left(\lambda_{i}^{(1)}-1\right) \cos \left(\left(\lambda_{i}^{(1)}-3\right) \theta\right)\right] \cos (\theta)} \\
+\left[\left(\lambda_{i}^{(1)}-1\right) \operatorname{sen}\left(\left(\lambda_{i}^{(1)}-3\right) \theta\right)+Q_{i}^{(1)}\left(\lambda_{i}^{(1)}+1\right) \operatorname{sen}\left(\left(\lambda_{i}^{(1)}-1\right) \theta\right)\right] \operatorname{sen}(\theta) \\
{\left[\left(\lambda_{i}^{(1)}-1\right) \operatorname{sen}\left(\left(\lambda_{i}^{(1)}-3\right) \theta\right)+Q_{i}^{(1)}\left(\lambda_{i}^{(1)}+1\right) \operatorname{sen}\left(\left(\lambda_{i}^{(1)}-1\right) \theta\right)\right] \cos (\theta)} \\
+\left[\left(2+Q_{i}^{(1)}\left(\lambda_{i}^{(1)}+1\right)\right) \cos \left(\left(\lambda_{i}^{(1)}-1\right) \theta\right)+\left(\lambda_{i}^{(1)}-1\right) \cos \left(\left(\lambda_{i}^{(1)}-3\right) \theta\right)\right] \operatorname{sen}(\theta)
\end{array}\right\}
$$

e para $\mathrm{n}=2$ (parte anti-simétrica)

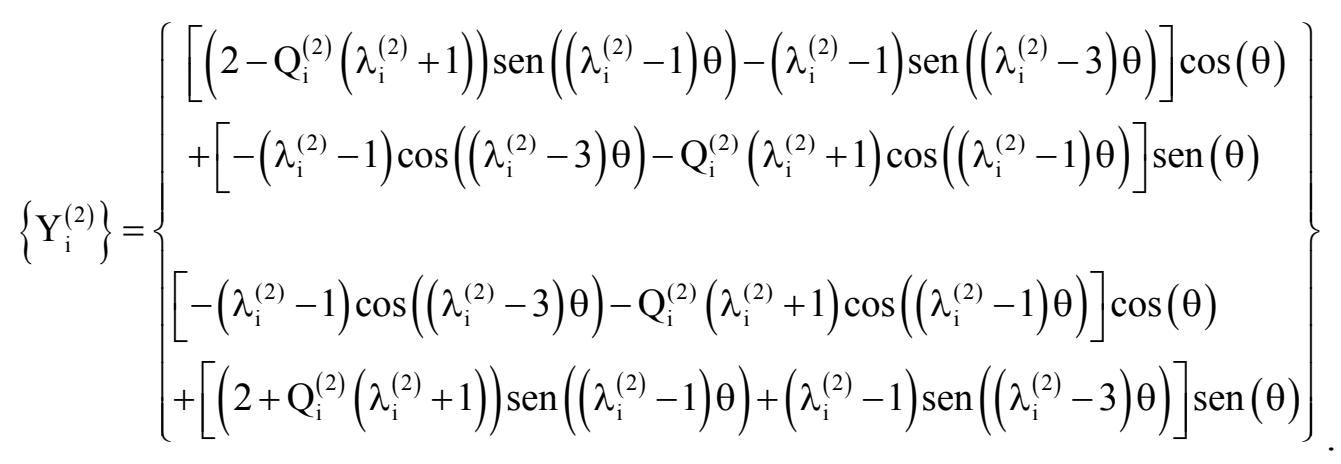

Similarmente, o vetor de tração correspondente ao campo de deslocamento $\overrightarrow{\mathrm{v}}_{-j}$ em $\Gamma_{\rho}$ é:

$$
\left\{\mathrm{T}_{\mathrm{i}}^{\left(\mathrm{v}_{-\mathrm{j}}\right)}\right\}=\lambda_{-\mathrm{j}}^{(\mathrm{m})} \rho^{\lambda_{-\mathrm{j}}^{(\mathrm{m})}-1}\left\{\mathrm{Y}_{-\mathrm{j}}^{(\mathrm{m})}\right\}
$$

$\left\{Y_{-j}^{(m)}\right\}$ é escrita na forma das equações (3.23) e (3.24), modificando apenas o uso de auto-valores negativos.

Utilizando-se os campos de deslocamento (3.20), as trações (3.21) e (3.25) e a integral independente do caminho (3.17) no contorno $\Gamma_{\rho}$, tem-se:

$$
I_{\Gamma_{p}}\left(\vec{u}_{i}, \vec{v}_{-j}\right)=\rho^{\lambda_{i}^{(n)}+\lambda_{-j}^{(m)}} C_{i,-j}^{(m, n)} ; \quad(m, n=1,2)
$$

sendo:

$$
\mathrm{C}_{\mathrm{i},-\mathrm{j}}^{(\mathrm{m}, \mathrm{n})}=\frac{1}{2 \mathrm{G}} \int_{-\alpha / 2}^{\alpha / 2}\left(\lambda_{\mathrm{i}}^{(\mathrm{n})}\left\{\mathrm{Y}_{\mathrm{i}}^{(\mathrm{n})}(\theta)\right\}^{\mathrm{T}}\left\{\Psi_{-\mathrm{j}}^{(\mathrm{m})}(\theta)\right\}-\lambda_{-\mathrm{j}}^{(\mathrm{m})}\left\{\mathrm{Y}_{-\mathrm{j}}^{(\mathrm{m})}(\theta)\right\}^{\mathrm{T}}\left\{\Psi_{\mathrm{i}}^{(\mathrm{n})}(\theta)\right\} d \mathrm{~d} \theta\right.
$$


$I_{\Gamma_{\rho}}$ (3.26) é uma integral independente do caminho, portanto é independente do raio de integração $\rho$ (Figura 3.1). Algumas observações podem ser feitas com relação ao valor final da integral (3.27):

- Se $\lambda_{i}^{(n)} \neq\left|\lambda_{-j}^{(m)}\right|$ a equação (3.26) só será independente de $\rho$ se, e somente se, $\mathrm{C}_{\mathrm{i},-\mathrm{j}}^{(\mathrm{m}, \mathrm{n})}=0$;

- Se $\lambda_{i}^{(n)}=\left|\lambda_{-j}^{(m)}\right|$ e $m \neq n$ o integrando é a soma dos produtos internos das partes simétrica e anti-simétrica da solução, portanto $C_{i,-j}^{(m, n)}=0$ e, conseqüentemente, a equação (3.26) será independente de $\rho$.

- Se $\lambda_{\mathrm{i}}^{(\mathrm{n})}=-\lambda_{-\mathrm{j}}^{(\mathrm{m})}$, para $\mathrm{m}=\mathrm{n}$ e $\mathrm{i}=\mathrm{j}$ no caso anterior, $\mathrm{C}_{\mathrm{i},-\mathrm{j}}^{(\mathrm{m}, \mathrm{n})}$ pode ser arbitrário e a (3.26) será independente do caminho.

Assim, para que a equação (3.26) seja independente do caminho, das propriedades da equação (3.27), tem-se:

$$
C_{i,-j}^{(m, n)}=\left\{\begin{array}{l}
\text { Se } i=j \text { e } m=n \\
c_{i}^{(m)}(\alpha)=C_{i,-i}^{(m, m)}, \\
\text { senão } \\
0
\end{array}\right.
$$

\subsection{Extração dos fatores de intensidade de tensão}

Utilizando-se as formulações apresentadas nas Seções 3.1 e 3.2 é possível desenvolver algoritmos para extração dos fatores de intensidade de tensão. Assim, Szabó e Babuška (1988) apresentaram o método da integral de contorno (MIC) e o método da função cutoff (MFC) para a determinação dos coeficientes da expansão assintótica $\mathrm{A}_{\mathrm{i}}^{(\mathrm{m})}(\mathrm{i}=1,2, \ldots ; \mathrm{m}, \mathrm{n}=1,2) \quad(2.41)$. No presente trabalho, enfatiza-se a extração dos primeiros termos da expansão assintótica, que são equivalentes aos fatores de intensidade de tensão. A principal diferença entre os métodos MIC e MFC está na escolha das funções extratoras utilizadas. Essas e outras particularidades dos métodos são apresentadas nas Seções 3.3.1 e 3.3.2. 


\subsubsection{Método da Integral de Contorno (MIC)}

Considera-se, primeiramente, o caminho de integração $\Gamma_{\rho}$ como um arco circular de raio $\rho$ centrado no vértice da região reentrante do domínio (Figura 3.2). Assume-se $\rho$ suficientemente próximo ao vértice, de maneira que a solução exata $\left\{\mathrm{u}_{\mathrm{EX}}\right\}$ possa ser representada por:

$$
\left\{\mathrm{u}_{\mathrm{EX}}\right\}=\sum_{\mathrm{i}=1}^{\infty} \sum_{\mathrm{n}=1}^{2} \frac{\mathrm{A}_{\mathrm{i}}^{(\mathrm{n})}}{2 \mathrm{G}} \mathrm{r}^{\lambda_{\mathrm{i}}^{(\mathrm{n})}}\left\{\Psi_{\mathrm{i}}^{(\mathrm{n})}\right\} .
$$

O respectivo vetor tração para (3.29) pode ser escrito como:

$$
\left\{T^{\left(u_{\mathrm{EX}}\right)}\right\}=\sum_{\mathrm{i}=1}^{\infty} \sum_{\mathrm{n}=1}^{2} \mathrm{~A}_{\mathrm{i}}^{(\mathrm{n})} \lambda_{\mathrm{i}}^{(\mathrm{n})} \rho^{\lambda_{\mathrm{i}}^{(\mathrm{n})}-1}\left\{\mathrm{Y}_{\mathrm{i}}^{(\mathrm{n})}\right\}
$$

sendo $\lambda_{i}^{(1)}>0$ e $\lambda_{i}^{(2)}>0$ autovalores reais e simples.

Define-se também o campo de deslocamento para um problema auxiliar:

$$
\left\{\mathrm{W}_{-\mathrm{j}}^{(\mathrm{m})}\right\}=\frac{\mathrm{A}_{-\mathrm{j}}^{(\mathrm{m})}}{2 \mathrm{G}} \mathrm{r}^{\lambda_{-\mathrm{j}}^{(\mathrm{m})}}\left\{\Psi_{-\mathrm{j}}^{(\mathrm{m})}\right\}
$$

$\left\{\mathrm{W}_{-\mathrm{j}}^{(\mathrm{m})}\right\}$ é composta por autovalores negativos $\left(\lambda_{-\mathrm{j}}^{(\mathrm{m})}=-\lambda_{\mathrm{j}}^{(\mathrm{m})}\right) .\left\{\mathrm{W}_{-\mathrm{j}}^{(\mathrm{m})}\right\}$ não é um campo de deslocamento admissível em $\Omega$, porém é admissível em $\Omega^{*}$ (Seção 3.1) (Figura 3.1). No contorno $\Gamma_{\rho}$ o vetor tração, referente ao campo $\left\{\mathrm{w}_{-\mathrm{j}}^{(\mathrm{m})}\right\}(3.31)$, é:

$$
\left\{T_{-j}^{\left(w_{-j}^{(m)}\right)}\right\}=A_{-j}^{(m)} \lambda_{-j}^{(m)} \rho^{\lambda_{-j}^{(m)}-1}\left\{Y_{-j}^{(m)}\right\}
$$

Substituindo-se os campos $\left\{\mathrm{u}_{\mathrm{EX}}\right\}$ (3.29) e $\left\{\mathrm{w}_{-\mathrm{j}}^{(\mathrm{m})}\right\}$ (3.31) e suas respectivas trações ((3.30) e (3.32)) na integral (3.17), tem-se:

$$
I_{\Gamma_{p}}\left(u_{E X}, W_{-j}^{(m)}\right)=A_{-j}^{(m)} \sum_{i=1}^{\infty} \sum_{n=1}^{2} A_{i}^{(n)} \rho^{\lambda_{i}^{(n)}+\lambda_{-j}^{(m)}} C_{i,-j}^{(n, m)}
$$


utilizando-se a ortogonalidade (3.28) e sabendo-se que $\lambda_{-\mathrm{j}}^{(\mathrm{m})}=-\lambda_{\mathrm{j}}^{(\mathrm{m})}$ chega-se a

$$
I_{\Gamma_{\rho}}\left(u_{E X}, w_{-j}^{(m)}\right)=A_{-j}^{(m)} A_{j}^{(m)} c_{j}^{(m)}(\alpha)
$$

O coeficiente $c_{j}^{(m)}(\alpha)$ pode ser definido analiticamente (Seção 3.4). Assim, para

$$
\mathrm{A}_{-\mathrm{j}}^{(\mathrm{m})}=1 / \mathrm{c}_{\mathrm{j}}^{(\mathrm{m})}(\alpha)
$$

e considerando-se a independência do caminho (3.17) em $I_{\Gamma_{\rho}}\left(u_{E X}, w_{-j}^{(m)}\right)(3.34)$, tem-se:

$$
\mathrm{A}_{\mathrm{j}}^{(\mathrm{m})}=\int_{\Gamma^{*}}\left\{\mathrm{w}_{-\mathrm{j}}^{(\mathrm{m})}\right\}^{\mathrm{T}}\left\{\mathrm{T}^{\left(\mathrm{u}_{\mathrm{EX}}\right)}\right\} \mathrm{ds}-\int_{\Gamma^{*}}\left\{\mathrm{u}_{\mathrm{EX}}\right\}^{\mathrm{T}}\left\{\mathrm{T}^{\left(\mathrm{w}_{-\mathrm{j}}^{(\mathrm{m})}\right)}\right\} \mathrm{ds}
$$

para $\Gamma^{*}$ arbitrário. A função $\left\{\mathrm{w}_{-\mathrm{j}}^{(\mathrm{m})}\right\}$ é denominada de função extratora para $\mathrm{A}_{\mathrm{j}}^{(\mathrm{m})}$. Os valores para $\left\{\mathrm{u}_{\mathrm{EX}}\right\}$ e $\left\{\mathrm{T}^{\left(\mathrm{u}_{\mathrm{EX}}\right)}\right\}$ não são conhecidos. Entretanto, substituindo-se os valores $\left\{\mathrm{u}_{\mathrm{EX}}\right\}$ e $\left\{\mathrm{T}^{\left(\mathrm{u}_{\mathrm{EX}}\right)}\right\}$ por uma solução numérica $\left\{\mathrm{u}_{\mathrm{NUM}}\right\}$ e $\left\{\mathrm{T}^{\left(\mathrm{u}_{\mathrm{NUM}}\right)}\right\}$ é possível obter uma aproximação para os coeficientes da expansão assintótica $\mathrm{A}_{\mathrm{j}}^{(\mathrm{m})}$.

No presente trabalho, utiliza-se a solução numérica do método dos elementos finitos generalizados (MEFG) para a extração dos coeficientes $\mathrm{A}_{\mathrm{j}}^{(\mathrm{m})}$.

\subsubsection{Método da Função Cutoff (MFC)}

Assume-se que ambos os contornos $\Gamma_{1}^{*}$ e $\Gamma_{2}^{*}$ (Figura 3.1) são arcos circulares de raios $\rho_{1}$ e $\rho_{2}$, respectivamente $\left(\rho_{2}>\rho_{1}\right)$. Assim, define-se a função extratora para $\mathrm{A}_{\mathrm{j}}^{(\mathrm{m})}$ :

$$
\left\{\widetilde{\mathrm{W}}_{-\mathrm{j}}^{(\mathrm{m})}\right\}=\phi(\mathrm{r})\left\{\mathrm{W}_{-\mathrm{j}}^{(\mathrm{m})}\right\}
$$

sendo $\left\{\mathrm{W}_{-\mathrm{j}}^{(\mathrm{m})}\right\}$ semelhante a equação (3.31) e $\phi(\mathrm{r})$ chamada de função cutoff. Define-se a função cutoff por: 


$$
\phi(r)= \begin{cases}1 & \text { para } r \leq \rho_{1} \\ 1-3\left(\frac{r-\rho_{1}}{\rho_{2}-\rho_{1}}\right)^{2}+2\left(\frac{r-\rho_{1}}{\rho_{2}-\rho_{1}}\right)^{3} & \text { para } \rho_{2}<r<\rho_{1}, \\ 0 & \text { para } r \leq \rho_{2}\end{cases}
$$

a Figura 3.1 ilustra a representação gráfica da função cutoff.
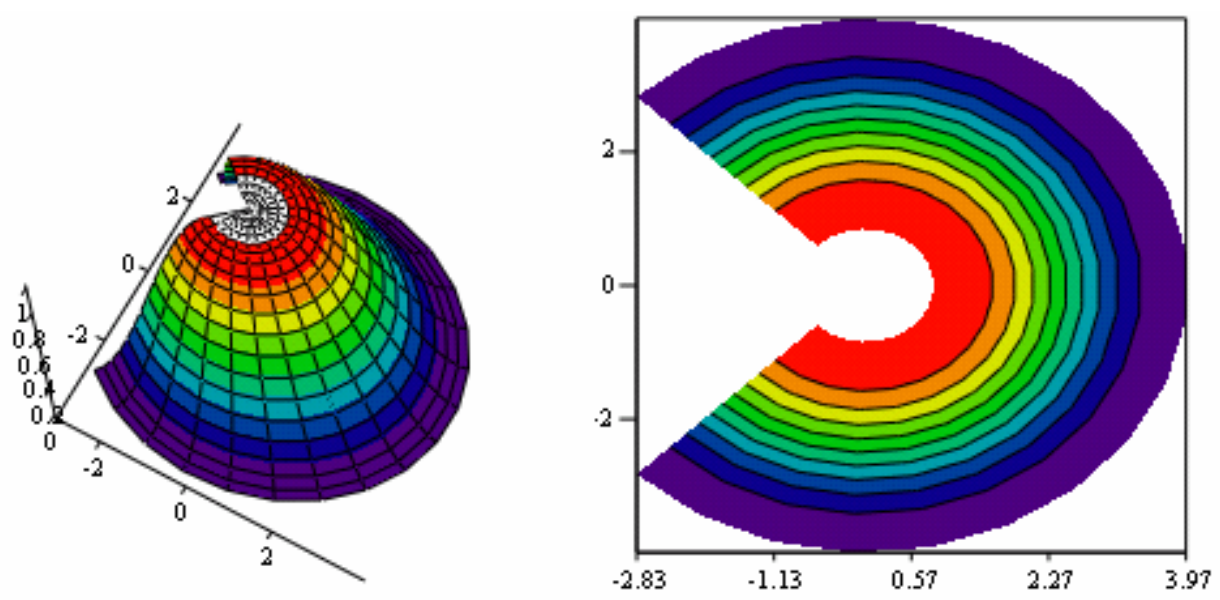

Figura 3.3 - Representação gráfica da função cutoff.

Nesse caso, a função extratora não satisfaz as condições de contorno na aresta reentrante e nem as equações de equilíbrio. Portanto, substitui-se a equação (3.17) por (3.18) para extrair os coeficientes $A_{j}^{(m)}$. Especificamente, adotando-se $A_{-j}^{(m)}=1 / c_{j}^{(m)}(\alpha)$, tem-se:

$$
\begin{aligned}
\mathrm{A}_{\mathrm{j}}^{(\mathrm{m})}= & \int_{\Gamma_{3}^{*}}\left(\mathrm{~T}_{\mathrm{x}}^{\left(\mathrm{w}_{-\mathrm{j}}^{(\mathrm{m})}\right)} \mathrm{u}_{\mathrm{x}}+\mathrm{T}_{\mathrm{y}}^{\left(\mathrm{w}_{-\mathrm{j}}^{(\mathrm{m})}\right)} \mathrm{u}_{\mathrm{y}}\right) \mathrm{ds}-\int_{\Gamma_{4}^{*}}\left(\mathrm{~T}_{\mathrm{x}}^{\left(\mathrm{w}_{-\mathrm{j}}^{(\mathrm{m})}\right)} \mathrm{u}_{\mathrm{x}}+\mathrm{T}_{\mathrm{y}}^{\left(\mathrm{w}_{-\mathrm{j}}^{(\mathrm{m})}\right)} \mathrm{u}_{\mathrm{y}}\right) \mathrm{ds} \\
& +\int_{\Omega^{*}}\left[\left(\frac{\partial \sigma_{\mathrm{x}}^{\left(\mathrm{w}_{-j}^{(\mathrm{m})}\right)}}{\partial \mathrm{x}}+\frac{\partial \tau_{x y}^{\left(\mathrm{w}_{-j}^{(\mathrm{m})}\right)}}{\partial \mathrm{y}}\right) \mathrm{u}_{\mathrm{x}}+\left(\frac{\partial \tau_{\mathrm{xy}}^{\left(\mathrm{w}_{-j}^{(\mathrm{m})}\right)}}{\partial \mathrm{x}}+\frac{\partial \sigma_{\mathrm{y}}^{\left(\mathrm{w}_{-j}^{(\mathrm{m})}\right)}}{\partial \mathrm{y}}\right) \mathrm{u}_{\mathrm{y}}\right] \mathrm{dxdy}
\end{aligned}
$$

As integrais de contorno em $\Gamma_{3}^{*}$ e $\Gamma_{4}^{*}$ devem ser calculadas no sentido antihorário com relação ao domínio $\Omega^{*}$ (Figura 3.1).

A função cutoff modifica a função extratora de tal maneira que: 
- As características da função extratora são preservadas no contorno mais próximo ao vértice $\left(\mathrm{em} \Gamma_{1}^{*}\right.$ onde $\left.\phi(\mathrm{r})=1\right)$;

- A função cutoff é uma função suave tal que a solução do problema auxiliar correspondente a $\left\{\widetilde{\mathrm{w}}_{-\mathrm{j}}^{(\mathrm{m})}\right\}$ pode ser aproximada de maneira satisfatória pelo MEF e pelo MEFG;

- $\left\{\widetilde{\mathrm{W}}_{-\mathrm{j}}^{(\mathrm{m})}\right\}$ e suas derivadas são nulas no contorno $\Gamma_{2}^{*}$. Portanto, as integrais de contorno em $\Gamma_{2}^{*}$ são nulas, dispensando o cálculo das tensões e trações obtidas com o método numérico utilizado. No MFC utiliza-se a solução numérica apenas para os deslocamentos. Por essa razão o MFC é mais preciso do que o MIC. Qualquer função suave que possua as mesmas características da função cutoff pode ser utilizada no MFC para compor a função extratora.

Algumas transformações de coordenadas necessárias para a implementação computacional do MFC podem ser encontradas no Apêndice C.

\subsection{Coeficiente da função extratora}

Utilizando-se a equação (3.28), as equações (3.23) e (3.24), que compõem o vetor tração ao longo do contorno $\Gamma_{\rho}$ (Figura 3.2), os autovalores e as autofunções, apresentados na Seção 2, é possível desenvolver a integral para o cálculo dos coeficientes $c_{i}^{(m)}(\alpha)$. Tais coeficientes definem os campos de deslocamento que são utilizados como funções extratoras no método da integral de contorno (MIC) e no método da função cutoff (MFC)

A Tabela 3.1 apresenta os valores $\mathrm{c}_{1}^{(\mathrm{m})}(\alpha)$ para os ângulos $\alpha$ (Figura 3.1) de maior importância do ponto de vista de engenharia. 
Tabela 3.1 - Valores de $\mathrm{c}_{1}^{(\mathrm{m})}(\alpha)$ para os Modos de deformação I e II para vértice com ângulo $\alpha$.

\begin{tabular}{ccc}
\hline$\alpha$ & $\mathrm{c}_{1}^{(1)}(\alpha)$ & $\mathrm{c}_{1}^{(2)}(\alpha)$ \\
\hline $360^{\circ}$ & $-2 \pi(1+\kappa) \frac{1}{2 \mathrm{G}}$ & $-2 \pi(1+\kappa) \frac{1}{2 \mathrm{G}}$ \\
$270^{\circ}$ & $-5,393243(1+\kappa) \frac{1}{2 \mathrm{G}}$ & $-1,760617(1+\kappa) \frac{1}{2 \mathrm{G}}$ \\
$240^{\circ}$ & $-5,430248(1+\kappa) \frac{1}{2 \mathrm{G}}$ & $2,295247(1+\kappa) \frac{1}{2 \mathrm{G}}$ \\
$225^{\circ}$ & $-5,604299(1+\kappa) \frac{1}{2 \mathrm{G}}$ & $5,832225(1+\kappa) \frac{1}{2 \mathrm{G}}$ \\
$210^{\circ}$ & $-5,859722(1+\kappa) \frac{1}{2 \mathrm{G}}$ & $8,776590(1+\kappa) \frac{1}{2 \mathrm{G}}$ \\
\hline
\end{tabular}

Na Tabela 3.1, $\kappa$ é a constante de Kolosov apresentada na Seção 2. As constantes $\mathrm{c}_{1}^{(1)}(\alpha)$ e $\mathrm{c}_{1}^{(2)}(\alpha)$ são os coeficientes da função extratora referentes aos modos I e II, respectivamente. No Apêndice B, descreve-se o procedimento para o desenvolvimento analítico das integrais utilizando o aplicativo MAPLE 7.

\subsection{Integral-J}

A Integral-J é a medida da taxa de energia disponibilizada nos casos onde os efeitos de plasticidade não são desprezados. Em problemas da elasticidade linear, pode ser demonstrado, para cada modo de deformação particular (Modos I, II e III), que a integral-J é também igual à taxa de energia disponibilizada para a propagação da fissura (RICE, 1968). Nos casos de estado plano de tensão ou estado plano de deformação a integral-J pode ser escrita na forma:

$$
J=\int_{\Gamma}\left(W d y-T \frac{\partial \vec{u}}{\partial x} d s\right),
$$

sendo $\Gamma$ um contorno em volta da ponta da fissura no sentido anti-horário (Figura 1.3) e W (3.41) a densidade de energia de deformação. 


$$
\mathrm{W}=\mathrm{W}(\mathrm{x}, \mathrm{y})=\frac{1}{2}\left(\sigma_{\mathrm{x}} \varepsilon_{\mathrm{x}}+\sigma_{\mathrm{y}} \varepsilon_{\mathrm{y}}+\tau_{\mathrm{xy}} \gamma_{\mathrm{xy}}\right)
$$

Na equação (3.40), T é o vetor tração (com componentes $T_{x}$ e $T_{y}$ ) ortogonal a $\Gamma$ (Figura 3.2), $\overrightarrow{\mathrm{u}}$ é o campo de deslocamento e ds é um elemento infinitesimal de $\Gamma$. Impõe-se o sistema de coordenadas de tal forma que a origem é localizada na ponta da fissura e a direção do eixo-x é paralela a aresta da fissura (Figura 1.3).

Considerando que a integral é calculada ao longo de um caminho circular de raio $\rho$, centrado na ponta da fissura (Figura 3.2), a equação (3.40) pode se escrita na forma:

$$
J=\int_{-\pi}^{\pi}\left(W \cos (\theta)-T_{x} \frac{\partial u_{x}}{\partial x}-T_{y} \frac{\partial u_{y}}{\partial x}\right) \rho d \theta
$$

No presente trabalho, utilizando a Integral-J, calcula-se a taxa de energia disponibilizada para a propagação da fissura e os fatores de intensidade de tensão (em modos puros) com o intuito de validar os resultados obtidos com os métodos MIC e MFC. Algumas transformações de coordenadas necessárias para a implementação numérica do método da Integral-J podem ser encontradas no Apêndice A. 


\section{Aspectos da formulação numérica dos métodos de extração}

A etapa de formulação numérica dos métodos da integral de contorno (MIC) e da função cutoff (MFC) consiste na avaliação numérica das integrais de contorno e de domínio apresentadas no Capítulo 3. Neste capítulo, apresentam-se os principais aspectos necessários para o desenvolvimento e implementação dos algoritmos para os métodos de extração utilizados no presente trabalho.

\subsection{Integração Numérica}

A integração numérica é utilizada quando uma integral definida na forma:

$$
\int_{a}^{b} f(x) d x,
$$

apresenta desenvolvimento analítico difícil ou impossível. Para esses casos e para problemas de integração mais gerais, nos quais apenas alguns valores do integrando são definidos em pontos distintos, ou até mesmo quando os valores dos integrandos são definidos apenas na forma numérica, faz-se o uso de aproximações para a integração.

Uma alternativa na avaliação numérica de integrais é encontrar uma função $\mathrm{g}(\mathrm{x})$ que aproxime $f(x)$ de forma satisfatória e que seja de fácil integração. Polinômios interpoladores, como os polinômios Legendre e os polinômios de Hermite, são funções que possuem essas características. 
$\mathrm{Na}$ integração numérica, a localização dos pontos utilizados na integração discretizada, o grau e o tipo do polinômio interpolador são parâmetros que caracterizam o método que está sendo aplicado.

Os métodos clássicos de integração podem ser divididos em dois grupos: os métodos de Newton-Cotes, como o método da regra trapezoidal e o método da regra de Simpson, que aplicam os valores das funções com pontos de integração igualmente espaçados e as quadraturas Gaussianas, como a quadratura de Gauss-Legendre e a quadratura de Gauss-Hermite, que aplicam os pontos de integração com espaçamentos diferentes, determinados por certas propriedades dos polinômios ortogonais.

\subsubsection{Quadratura Gaussiana (Gauss-Legendre)}

No cálculo das integrais dos métodos de extração, utiliza-se o método de integração numérica de Gauss-Legendre. Procedimentos especiais de integração não são utilizados, pois o domínio de integração não contém a extremidade da fissura.

\subsubsection{Integração em domínios unidimensionais}

No método da quadratura Gaussiana, aproxima-se o valor de uma dada integral em um intervalo normalizado $[-1 ; 1]$ pela integral do polinômio interpolador da função nesse trecho. Para casos unidimensionais, a integral é avaliada por meio do somatório do valor da função em determinados pontos $\xi_{\mathrm{i}}$ (abscissas) multiplicados pelo fator de ponderação $\omega_{i}$ (pesos), como apresentado na equação (4.2).

$$
\int_{-1}^{1} \mathrm{f}(\xi) \mathrm{d} \xi \approx \sum_{\mathrm{i}=1}^{\mathrm{N}} \mathrm{f}\left(\xi_{\mathrm{i}}\right) \omega_{\mathrm{i}},
$$

cada abscissa $\xi_{\mathrm{i}}$ e o seu respectivo peso $\omega_{\mathrm{i}}$ são conhecidos como pontos de Gauss.

Os valores das abscissas $\xi_{\mathrm{i}}$ e o seus respectivos pesos $\omega_{\mathrm{i}}$ são anti-simétricos e simétricos com relação a $\xi=0$, respectivamente. Para um número de pontos ímpar as abscissas $\xi_{\mathrm{i}}$ incluem o ponto $\xi=0$. Um típico domínio de integração de Gauss para o caso unidimensional é apresentado na Figura 4.1. 


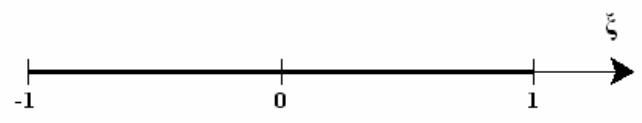

Figura 4.1 - Domínio unidimensional para integração Gaussiana.

Na quadratura de Gauss-Legendre, os valores das abscissas $\xi_{i}$ são definidos pelas raízes dos polinômios de Legendre. Quando o integrando é um polinômio de grau $2 \mathrm{~N}+1$, sendo $\mathrm{N}$ o número de pontos de Gauss utilizados na integração, o erro na integração numérica depende apenas da precisão utilizada no cálculo das abscissas $\xi_{\mathrm{i}} \mathrm{e}$ dos seus respectivos pesos $\omega_{i}$. Se a função integrando não for um polinômio, o erro existente na integração está relacionado com a quantidade de pontos de Gauss utilizados na integração.

\subsubsection{Integração em domínios bidimensionais}

As integrais bidimensionais avaliadas na forma numérica podem ser admitidas como uma combinação de duas integrais unidimensionais, como apresentado na equação (4.3):

$$
\int_{-1}^{1} \int_{-1}^{1} \mathrm{f}(\xi, \eta) \mathrm{d} \xi \mathrm{d} \eta \approx \sum_{\mathrm{i}=1}^{\mathrm{N}} \sum_{\mathrm{j}=1}^{\mathrm{M}} \mathrm{f}\left(\xi_{\mathrm{i}}, \eta_{\mathrm{j}}\right) \omega_{\mathrm{i}}^{(\xi)} \omega_{\mathrm{j}}^{(\eta)}
$$

$\omega_{i}^{(\xi)}$ e $\omega_{j}^{(\eta)}$ representam os pesos referentes a $\xi_{i}$ e $\eta_{j}$, respectivamente. Todas as observações feitas para o caso unidimensional são válidas para o caso bidimensional. Essa abordagem é válida para domínios bidimensionais que podem ser mapeados para um quadrado.

Um domínio típico para integração Gaussiana bidimensional é apresentado na Figura 4.2. 


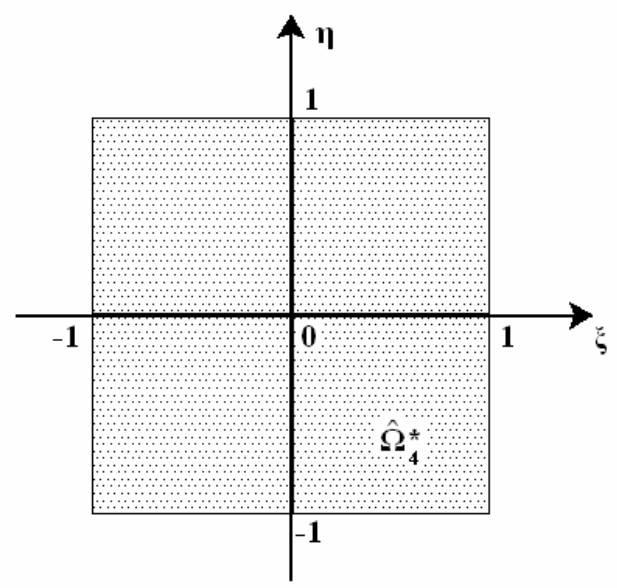

Figura 4.2 - Domínio bidimensional para integração Gaussiana.

\subsection{Formulação numérica dos métodos de extração}

Para aplicar o método de integração Gaussiana às integrais dos métodos da integral de contorno (MIC) e da função cutoff (MFC), apresentados no Capítulo 3, fazse necessário o desenvolvimento de algumas transformações de coordenadas. $\mathrm{O}$ uso de tais transformações de coordenadas promove generalizações na formulação numérica e na relação entre implementação do método de extração e metodologia adotada na solução do problema.

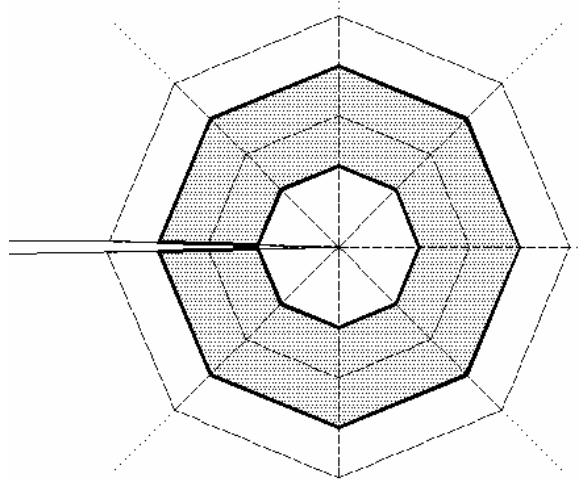

(a)

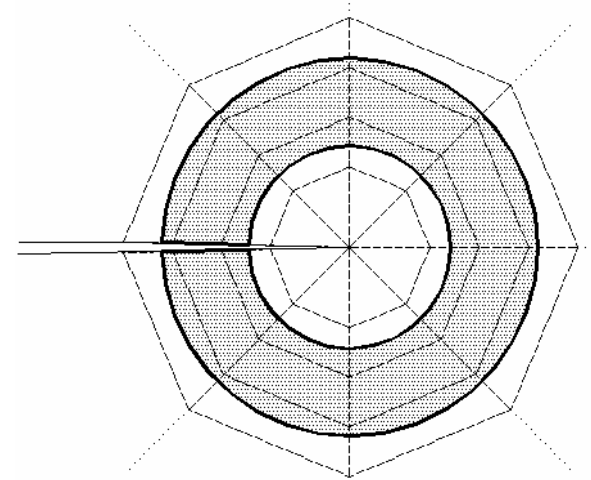

(b)

malha fissura

contorno

domínio

Figura 4.3 - Domínios para integração numérica em torno da ponta da fissura: (a) utilizando a discretização do problema e (b) independente da discretização. 
A implementação numérica de métodos de extração dos fatores de intensidade de tensão, geralmente, está associada à malha de elementos finitos utilizada na discretização do problema (MORAN; SHIH, 1987 a, b; NAHTA; MORAN, 1993; ORGAN, 1996; RAJU; SHIVAKUMAR, 1990). Nesses métodos de extração são utilizados anéis de elementos finitos em torno da extremidade da fissura (Figura 4.3 (a))

${ }^{2}$. No presente trabalho, desenvolve-se uma abordagem em que o processo de integração para os métodos de extração não utiliza os elementos da malha empregada na análise (Figura 4.3 (b)). Algumas vantagens desta abordagem são:

- As implementações em duas e em três dimensões podem ser feitas compartilhando boa parte do código computacional se uma linguagem orientada a objetos, como $\mathrm{C}++$, for utilizada.

- A implementação é completamente independente do tipo de elemento utilizado (quadrilátero, triangular, tetraédrico, etc), da discretização, da ordem polinomial do elemento, etc.

- A implementação é ideal para ser usada em propagação de fraturas utilizando o MEFG.

- A implementação pode ser utilizada com qualquer método numérico que forneça uma solução aproximada do problema da elasticidade.

As transformações utilizadas para as integrais de contorno e de área, seus respectivos jacobianos e a formulação numérica para cada método são apresentados nas Seções 4.2.1 e 4.2.2.

\subsubsection{Método da integral de contorno (MIC)}

\subsubsection{Transformação de coordenadas}

Para a implementação do MIC, utilizando quadratura Gaussiana é necessário desenvolver uma transformação de coordenadas que conduza pontos do domínio normalizado $\hat{\Gamma}^{4}$ para o domínio em coordenadas globais $\Gamma$ (Figura 4.4). Essa

\footnotetext{
${ }^{2}$ As malhas apresentadas na Figura 4.3 servem apenas para ilustração. Em geral, na extração de fatores de intensidade de tensão (RAJU; SHIVAKUMAR, 1990), utilizam-se várias camadas de elementos em torno da ponta da fissura, especialmente na versão h do MEF (SZABÓ; BABUŠKA, 1991).
} 
transformação de coordenadas também é utilizada na implementação do método da Integral-J (Seção 3.5).

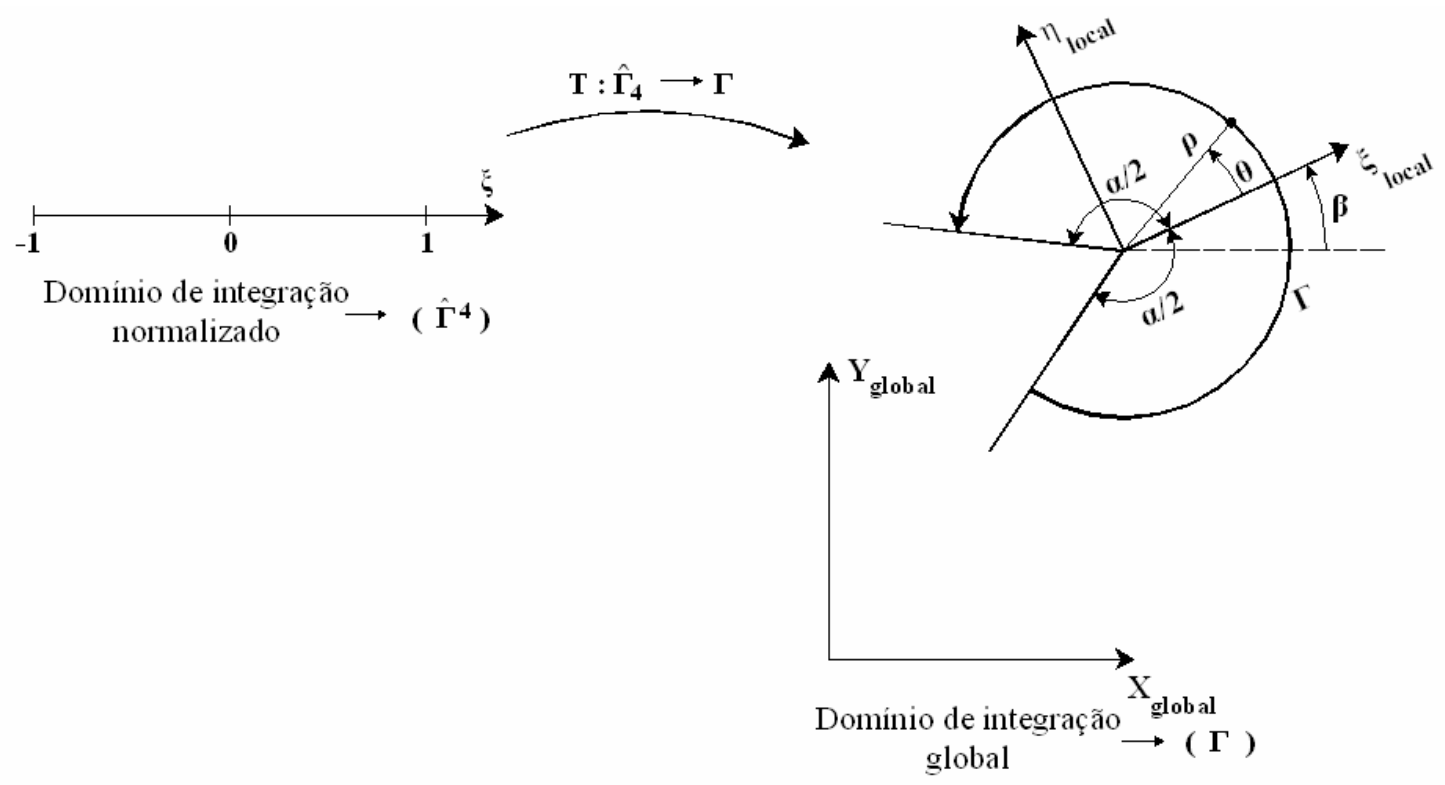

Figura 4.4 - Transformação de coordenadas (método da integral de contorno - MIC).

Para facilitar o entendimento, a formulação utilizada na mudança de coordenadas para integração numérica do método da integral de contorno (MIC) foi divida em quatro etapas. Tais etapas e a transformação final são descritas a seguir.

$\underline{\text { Passo I }}$

O primeiro passo é desenvolver um equacionamento que transforme as coordenadas de um sistema normalizado, com os eixos paralelos aos eixos globais, localizado no vértice da região reentrante $\hat{\Gamma}_{1}$, em coordenadas do sistema global $\Gamma$ (Figura 4.5). 


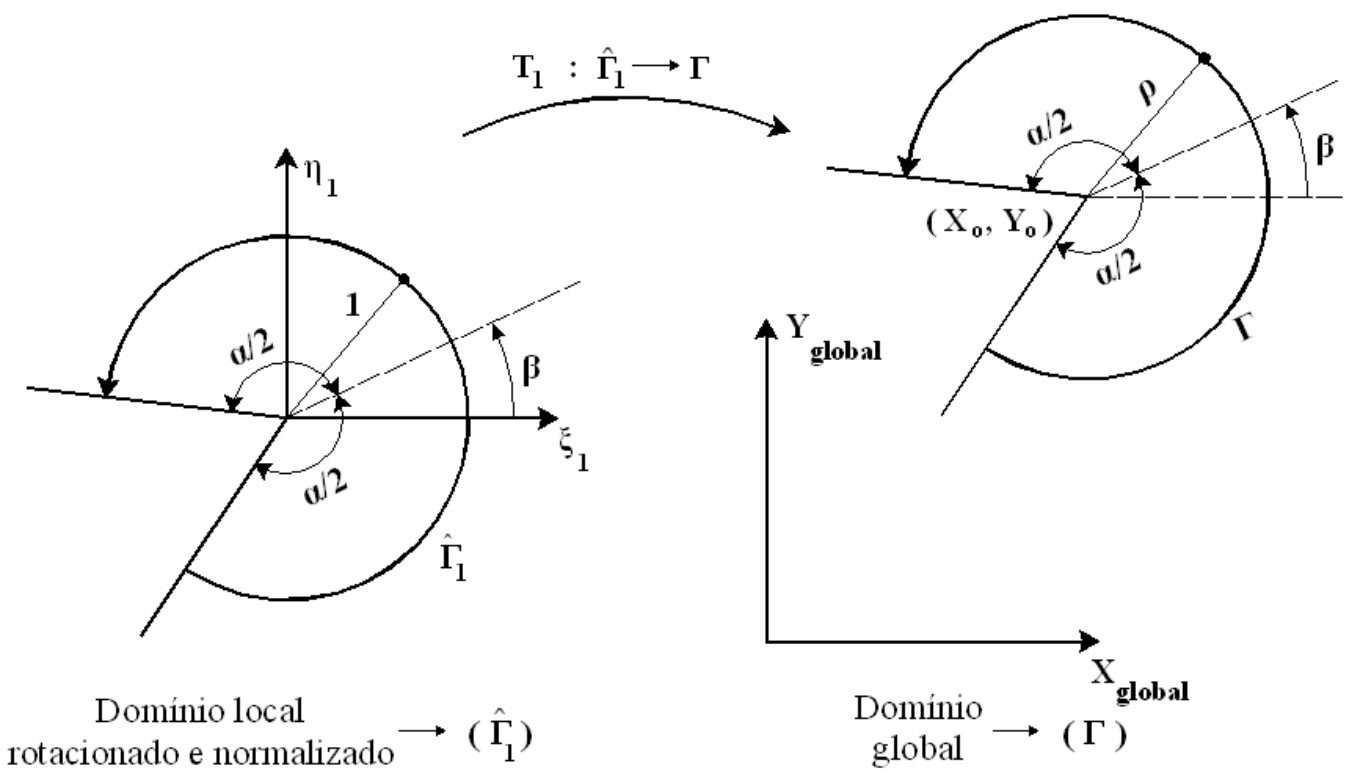

Figura 4.5 - Transformação de coordenadas (método da integral de contorno - MIC) Passo I

$\mathrm{Na}$ transformação do espaço $\hat{\Gamma}_{1}$ para o espaço $\Gamma$ utilizam-se as seguintes funções:

$$
\begin{aligned}
& \mathrm{T}_{1}: \hat{\Gamma}_{1} \rightarrow \Gamma \\
& \left(\begin{array}{l}
\mathrm{X} \\
\mathrm{Y}
\end{array}\right)=\rho\left(\begin{array}{l}
\xi_{1} \\
\eta_{1}
\end{array}\right)+\left(\begin{array}{l}
X_{\mathrm{o}} \\
Y_{\mathrm{o}}
\end{array}\right) .
\end{aligned}
$$

$\mathrm{X}_{\mathrm{o}}$ e $\mathrm{Y}_{\mathrm{o}}$ são as coordenadas globais do vértice da região reentrante, $\rho$ é o raio do arco de circular $\Gamma$ e as coordenadas $\xi_{1}$ e $\eta_{1}$ obedecem a equação do arco circular de raio unitário $\hat{\Gamma}_{1}$. Essa transformação representa uma translação e uma variação de escala nas coordenadas.

\section{Passo II}

Aplica-se uma rotação entre as coordenadas de $\hat{\Gamma}_{2}$ e $\hat{\Gamma}_{1}$ (Figura 4.6). 


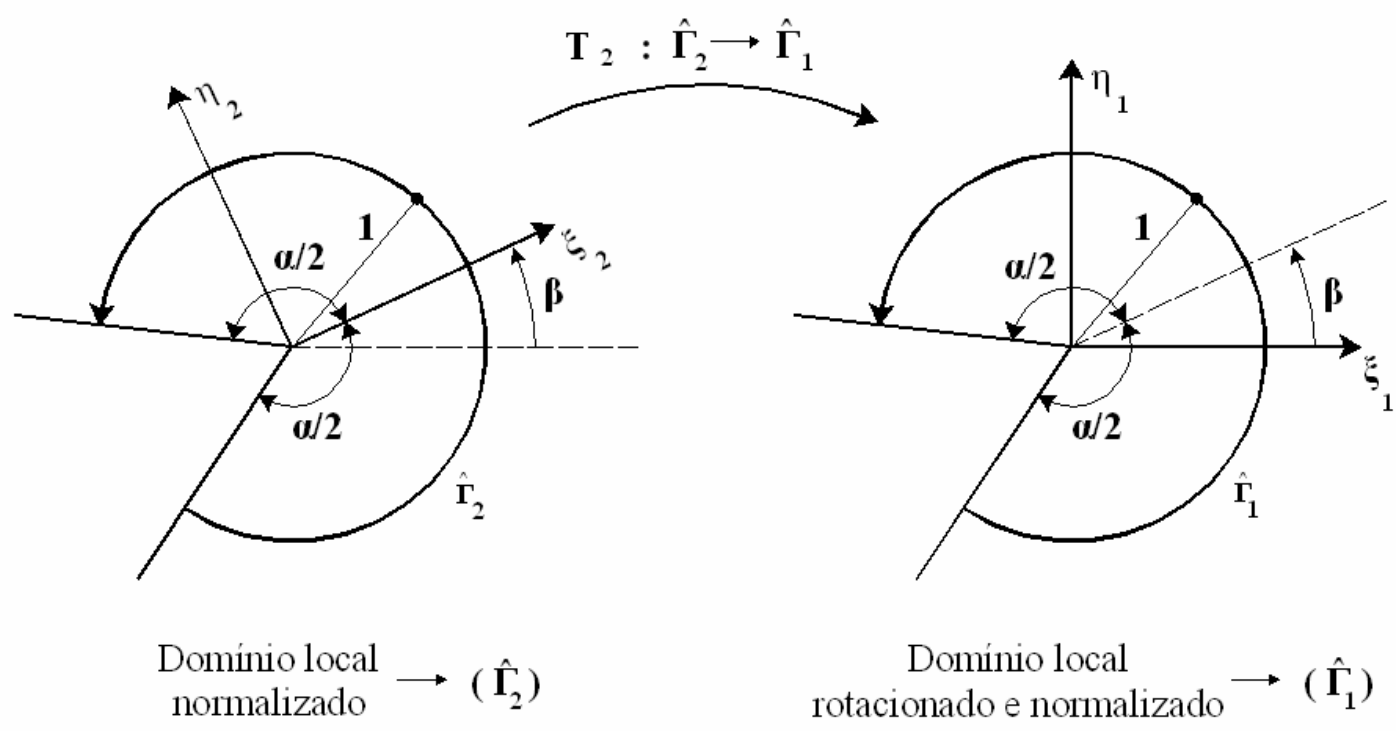

Figura 4.6 - Transformação de coordenadas (método da integral de contorno - MIC) Passo II.

As equações que possibilitam a rotação entre os espaços são:

$$
\begin{aligned}
& \mathrm{T}_{2}: \hat{\Gamma}_{2} \rightarrow \hat{\Gamma}_{1} \\
& \left(\begin{array}{l}
\xi_{1} \\
\eta_{1}
\end{array}\right)=\left[\begin{array}{cc}
\cos (\beta) & -\operatorname{sen}(\beta) \\
\operatorname{sen}(\beta) & \cos (\beta)
\end{array}\right]\left(\begin{array}{l}
\xi_{2} \\
\eta_{2}
\end{array}\right) .
\end{aligned}
$$

sendo $\beta$ o ângulo entre a bissetriz do ângulo do material $(\alpha)$ e o eixo horizontal de coordenadas globais (Figura 4.4).

\section{Passo III}

Aplica-se uma transformação entre as coordenadas locais $\xi_{2}$ e $\eta_{2}$ na ponta da fissura e as coordenadas $\mathrm{r}$ e $\theta$ representadas no plano cartesiano (Figura 4.7). 


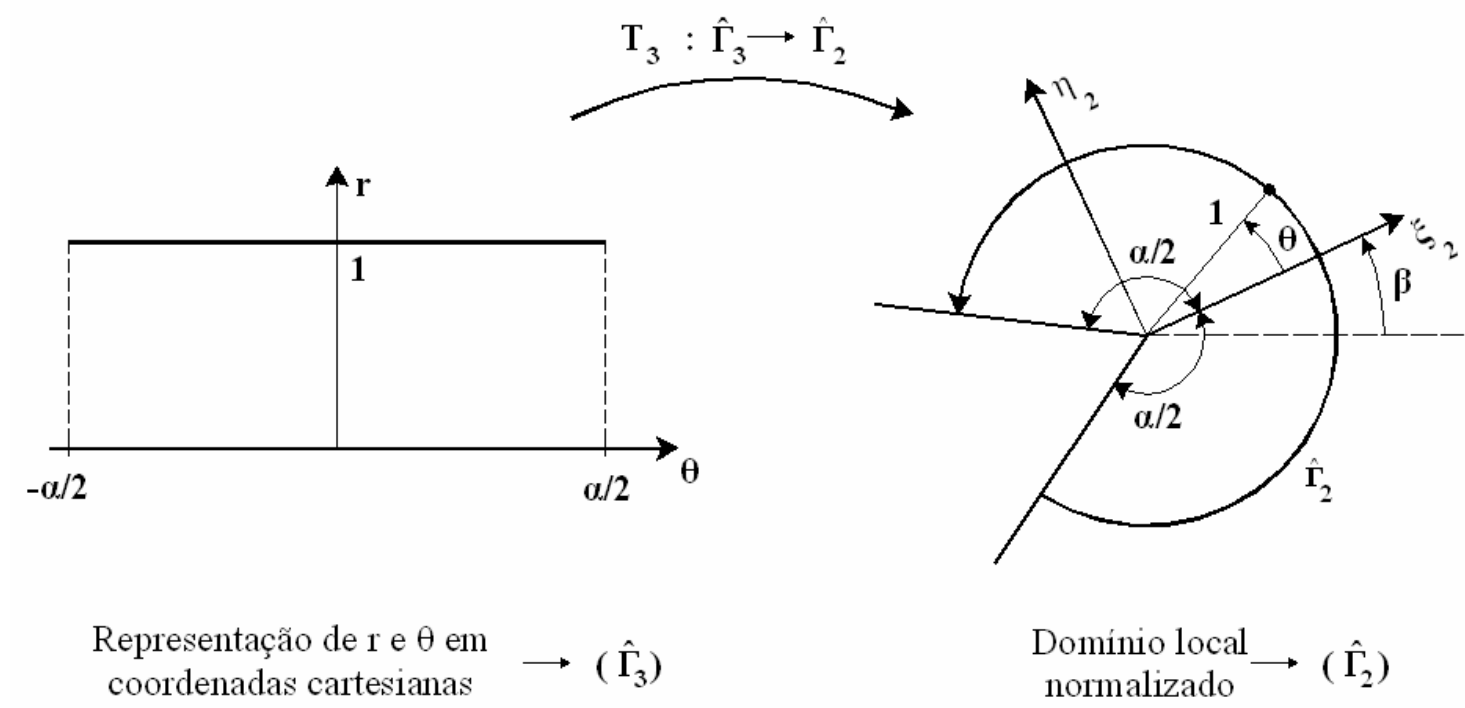

Figura 4.7 - Transformação de coordenadas (método da integral de contorno - MIC) Passo III.

As equações da referida transformação entre os espaços $\hat{\Gamma}_{3}$ e $\hat{\Gamma}_{2}$ são apresentadas a seguir:

$$
\begin{aligned}
& \mathrm{T}_{3}: \hat{\Gamma}_{3} \rightarrow \hat{\Gamma}_{2} \\
& \left(\begin{array}{l}
\xi_{2} \\
\eta_{2}
\end{array}\right)=\left(\begin{array}{l}
\cos (\theta) \\
\operatorname{sen}(\theta)
\end{array}\right) .
\end{aligned}
$$

\section{Passo IV}

Transformar as coordenadas do domínio de integração de Gauss $\left(\hat{\Gamma}_{4}\right)$ para a representação das coordenadas $\mathrm{r}$ e $\theta$ no plano cartesiano $\left(\hat{\Gamma}_{3}\right)$. 


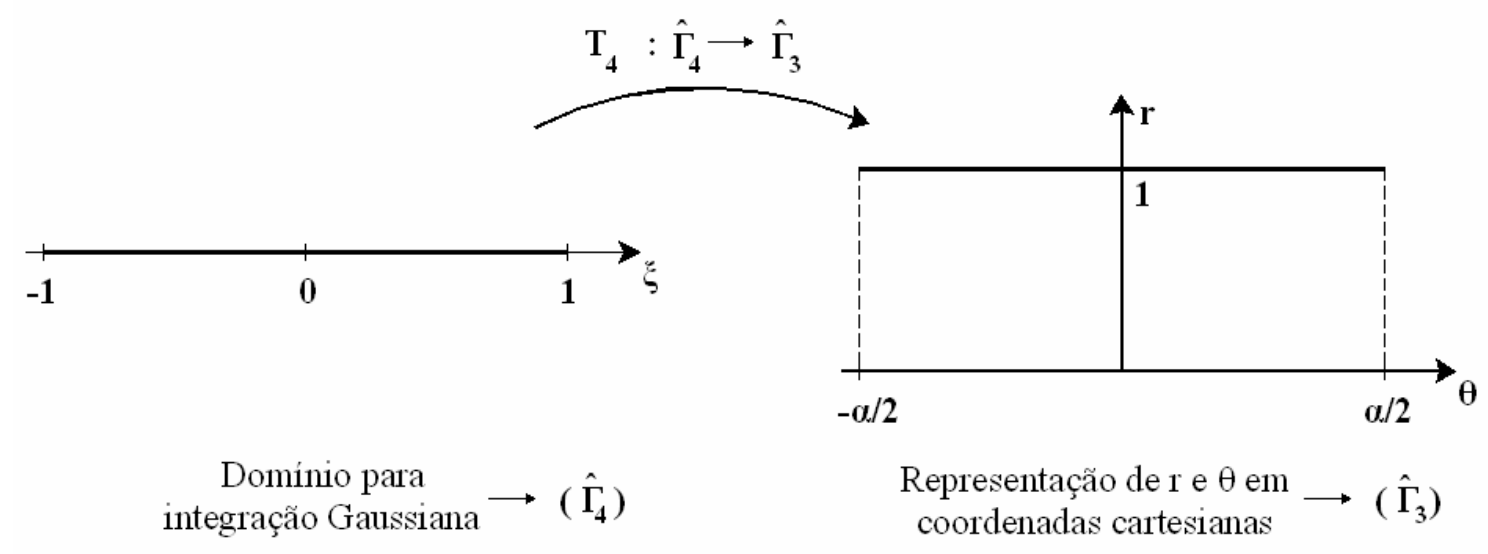

Figura 4.8 - Transformação de coordenadas (método da integral de contorno - MIC) Passo IV.

Sendo assim, a equação para a referida transformação é a seguinte:

$$
\begin{aligned}
& \mathrm{T}_{4}: \hat{\Gamma}_{4} \rightarrow \hat{\Gamma}_{3} \\
& \theta=\frac{\alpha}{2} \xi
\end{aligned}
$$

sendo $\xi$ a coordenada do domínio de integração de Gauss.

\section{Equacionamento Final}

$\mathrm{O}$ equacionamento final da transformação para a integração numérica do método da integral de contorno (MIC) é dado por:

$$
\begin{aligned}
& T=T_{1} \circ T_{2} \circ T_{3} \circ T_{4} \\
& \left(\begin{array}{l}
X \\
Y
\end{array}\right)=\rho\left[\begin{array}{cc}
\cos (\beta) & -\operatorname{sen}(\beta) \\
\operatorname{sen}(\beta) & \cos (\beta)
\end{array}\right]\left(\begin{array}{c}
\cos \left(\frac{\alpha}{2} \xi\right) \\
\operatorname{sen}\left(\frac{\alpha}{2} \xi\right)
\end{array}\right)+\left(\begin{array}{c}
X_{0} \\
Y_{0}
\end{array}\right) .
\end{aligned}
$$

O jacobiano da transformação é escrito na forma:

$$
\mathrm{ds}=\sqrt{\left(\frac{\mathrm{dX}}{\mathrm{d} \xi}\right)^{2}+\left(\frac{\mathrm{dY}}{\mathrm{d} \xi}\right)^{2}} \mathrm{~d} \xi \rightarrow \mathrm{ds}=\rho \frac{\alpha}{2} \mathrm{~d} \xi
$$




\subsubsection{Formulação numérica}

Utilizando a transformação de coordenadas, descrita na Seção 4.2.1.1, a equação do método da integral de contorno (MIC) é dada por:

$$
\begin{aligned}
& \mathrm{A}_{\mathrm{j}}^{(\mathrm{m})}=\int_{-1}^{1}\left\{\mathrm{w}_{-\mathrm{j}}^{(\mathrm{m})}(\mathrm{X}(\xi), \mathrm{Y}(\xi))\right\}^{\mathrm{T}}\left\{\mathrm{T}^{\left(\mathrm{u}_{\mathrm{EFG}}\right)}(\mathrm{X}(\xi), \mathrm{Y}(\xi))\right\} \rho \frac{\alpha}{2} \mathrm{~d} \xi \\
& -\int_{-1}^{1}\left\{\mathrm{u}_{\mathrm{EFG}}(\mathrm{X}(\xi), \mathrm{Y}(\xi))\right\}^{\mathrm{T}}\left\{\mathrm{T}^{\left(\mathrm{w}_{-j}^{(\mathrm{m})}\right)}(\mathrm{X}(\xi), \mathrm{Y}(\xi))\right\} \rho \frac{\alpha}{2} \mathrm{~d} \xi
\end{aligned}
$$

$\mathrm{u}_{\mathrm{EFG}}$ indica o campo de deslocamento solução do método de elementos finitos generalizados.

Simplificando e aplicando a quadratura Gaussiana, chega-se ao seguinte algoritmo:

$$
A_{j}^{(m)}=\sum_{i=1}^{n p G}\left(\left\{w_{-j}^{(m)}\left(\xi_{i}\right)\right\}^{T}\left\{T^{\left(u_{E F G}\right)}\left(\xi_{i}\right)\right\}-\left\{u_{E F G}\left(\xi_{i}\right)\right\}^{T}\left\{T^{\left(w_{-j}^{(m)}\right)}\left(\xi_{i}\right)\right\}\right) \omega_{i} \rho \frac{\alpha}{2}
$$

sendo npG o número de pontos de Gauss utilizados na integração numérica.

\subsubsection{Método da função cutoff (MFC)}

\subsubsection{Transformação de coordenadas}

Na formulação numérica do Método da função cutoff, utilizando-se quadratura Gaussiana, é necessário o uso de uma transformação de coordenadas, bem como o desenvolvimento do jacobiano dessa transformação. Desenvolve-se uma transformação que conduz pontos do domínio bidimensional de integração Gaussiana $\hat{\Omega}_{4}^{*}$ para o domínio de um setor circular vazado no centro, próximo a extremidade da região reentrante, em coordenadas globais $\Omega^{*}$ (Figura 4.9). No domínio $\Omega^{*}$, a variável R é definida no intervalo $\left[\rho_{1} ; \rho_{2}\right]$. 


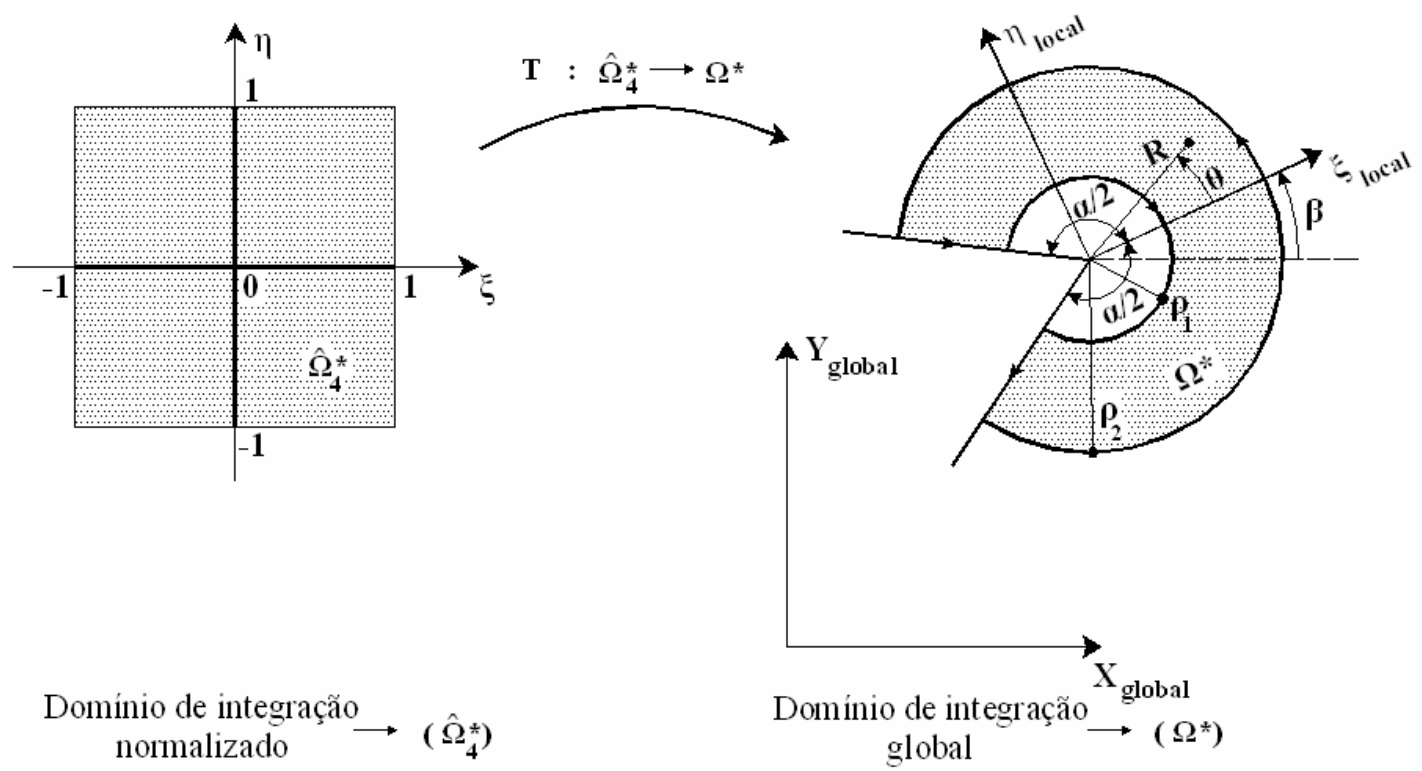

Figura 4.9 - Transformação de coordenadas para o método da função cutoff - MFC.

Para facilitar o entendimento, a formulação utilizada na mudança de coordenadas para integração numérica do método da função cutoff (MFC) foi divida em quatro etapas. Tais etapas e a transformação final são descritas a seguir.

\section{$\underline{\text { Passo I }}$}

O primeiro passo é desenvolver um equacionamento que transforme as coordenadas de um sistema normalizado, com os eixos paralelos aos eixos globais, localizado no vértice da região reentrante $\hat{\Omega}_{1}^{*}$, em coordenadas do sistema global $\Omega^{*}$ (Figura 4.10). 


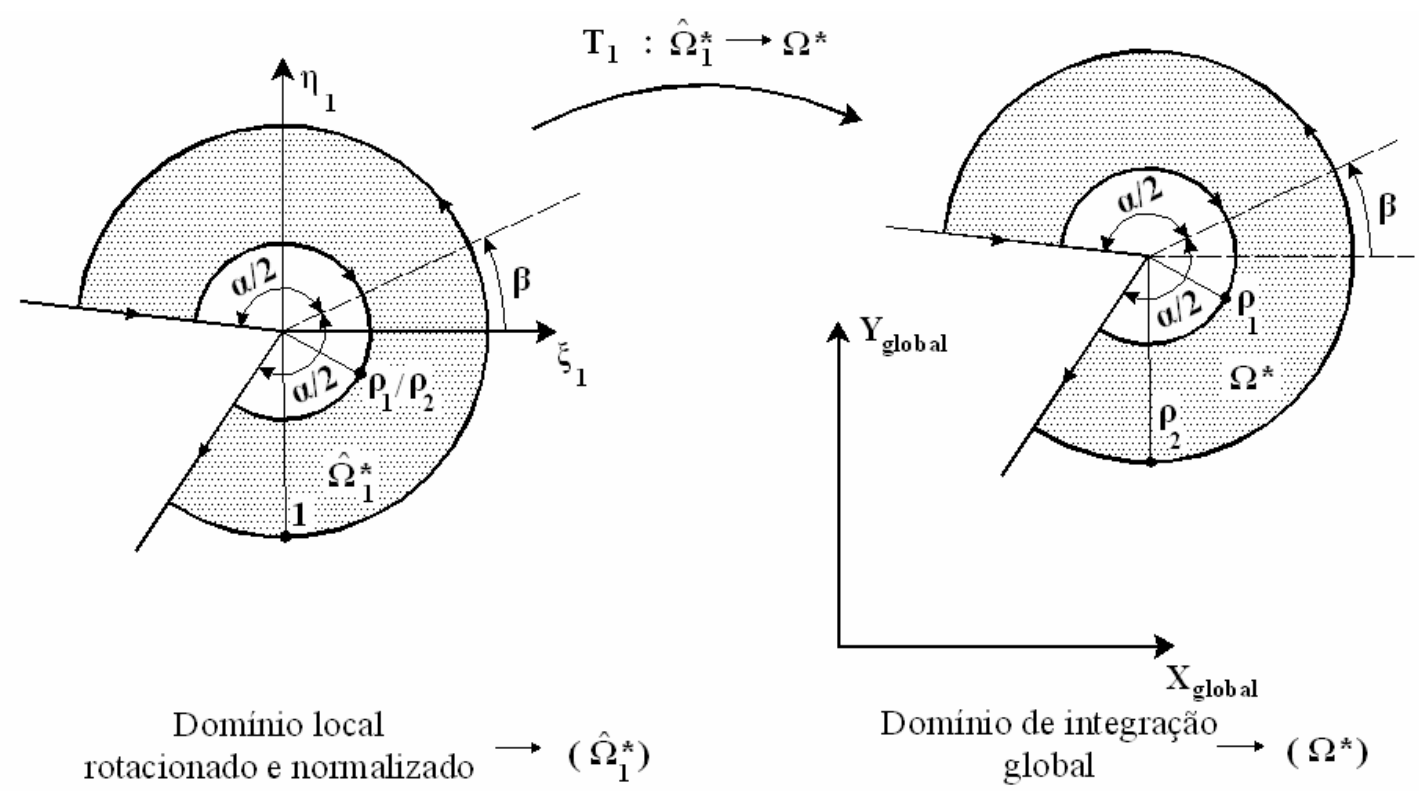

Figura 4.10 - Transformação de coordenadas para o método da função cutoff (MFC) Passo I.

As equações para a transformação apresentada na Figura 4.10 são:

$$
\begin{aligned}
& \mathrm{T}_{1}: \hat{\Omega}_{1}^{*} \rightarrow \Omega^{*} \\
& \left(\begin{array}{l}
\mathrm{X} \\
\mathrm{Y}
\end{array}\right)=\rho_{2}\left(\begin{array}{l}
\xi_{1} \\
\eta_{1}
\end{array}\right)+\left(\begin{array}{l}
\mathrm{X}_{\mathrm{o}} \\
\mathrm{Y}_{\mathrm{o}}
\end{array}\right) .
\end{aligned}
$$

O jacobiano para a transformação é dado por:

$$
J_{1}=\left|\begin{array}{ll}
\frac{\partial X}{\partial \xi_{1}} & \frac{\partial X}{\partial \eta_{1}} \\
\frac{\partial Y}{\partial \xi_{1}} & \frac{\partial Y}{\partial \eta_{1}}
\end{array}\right|=\left|\begin{array}{cc}
\rho_{2} & 0 \\
0 & \rho_{2}
\end{array}\right|=\left(\rho_{2}\right)^{2}
$$

\section{$\underline{\text { Passo II }}$}

Aplica-se uma rotação de coordenadas entre os espaços $\hat{\Omega}_{2}^{*}$ e $\hat{\Omega}_{1}^{*}$ : 


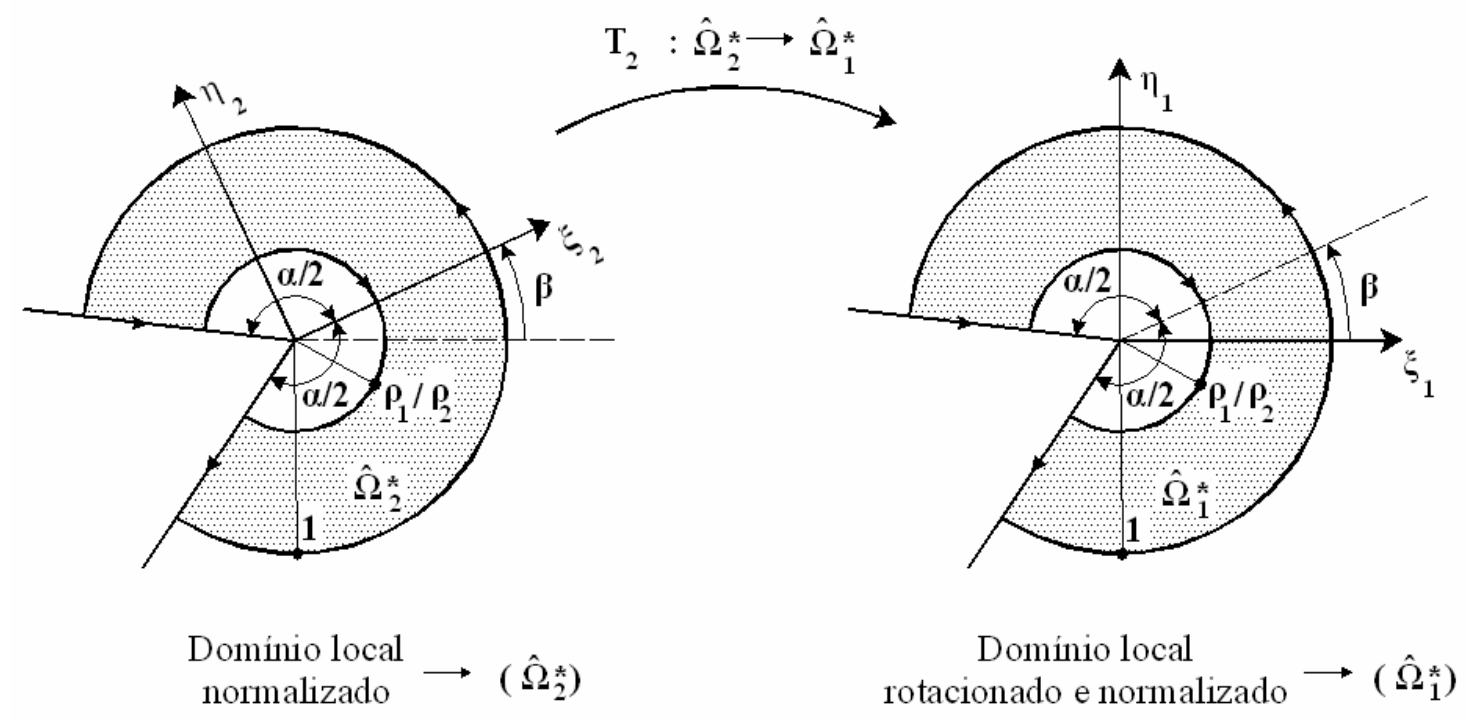

Figura 4.11 - Transformação de coordenadas para o método da função cutoff (MFC) Passo II.

As equações para a transformação entre os espaços $\hat{\Omega}_{2}^{*}$ e $\hat{\Omega}_{1}^{*}$ são apresentadas na forma:

$$
\begin{aligned}
& \mathrm{T}_{2}: \hat{\Omega}_{2}^{*} \rightarrow \hat{\Omega}_{1}^{*} \\
& \left(\begin{array}{l}
\xi_{1} \\
\eta_{1}
\end{array}\right)=\left[\begin{array}{cc}
\cos (\beta) & -\operatorname{sen}(\beta) \\
\operatorname{sen}(\beta) & \cos (\beta)
\end{array}\right]\left(\begin{array}{l}
\xi_{2} \\
\eta_{2}
\end{array}\right) .
\end{aligned}
$$

O jacobiano para a transformação $\mathrm{T}_{2}$ é dado por:

$$
J_{2}=\left|\begin{array}{ll}
\frac{\partial \xi_{1}}{\partial \xi_{2}} & \frac{\partial \xi_{1}}{\partial \eta_{2}} \\
\frac{\partial \eta_{1}}{\partial \xi_{2}} & \frac{\partial \eta_{1}}{\partial \eta_{2}}
\end{array}\right|=\left|\begin{array}{cc}
\cos (\beta) & -\operatorname{sen}(\beta) \\
\operatorname{sen}(\beta) & \cos (\beta)
\end{array}\right|=1
$$

sendo $\beta$ o ângulo entre a bissetriz do ângulo do material $(\alpha)$ e o eixo horizontal de coordenadas globais (Figura 4.9).

\section{$\underline{\text { Passo III }}$}

Desenvolve-se a transformação entre as coordenadas locais $\xi_{2}$ e $\eta_{2}$ na ponta da fissura e as coordenadas $\mathrm{r}$ e $\theta$ representadas no plano cartesiano (Figura 4.12). A variável $\mathrm{r}$ é definida no intervalo normalizado $\left[\rho_{1} / \rho_{2} ; 1\right]$. 


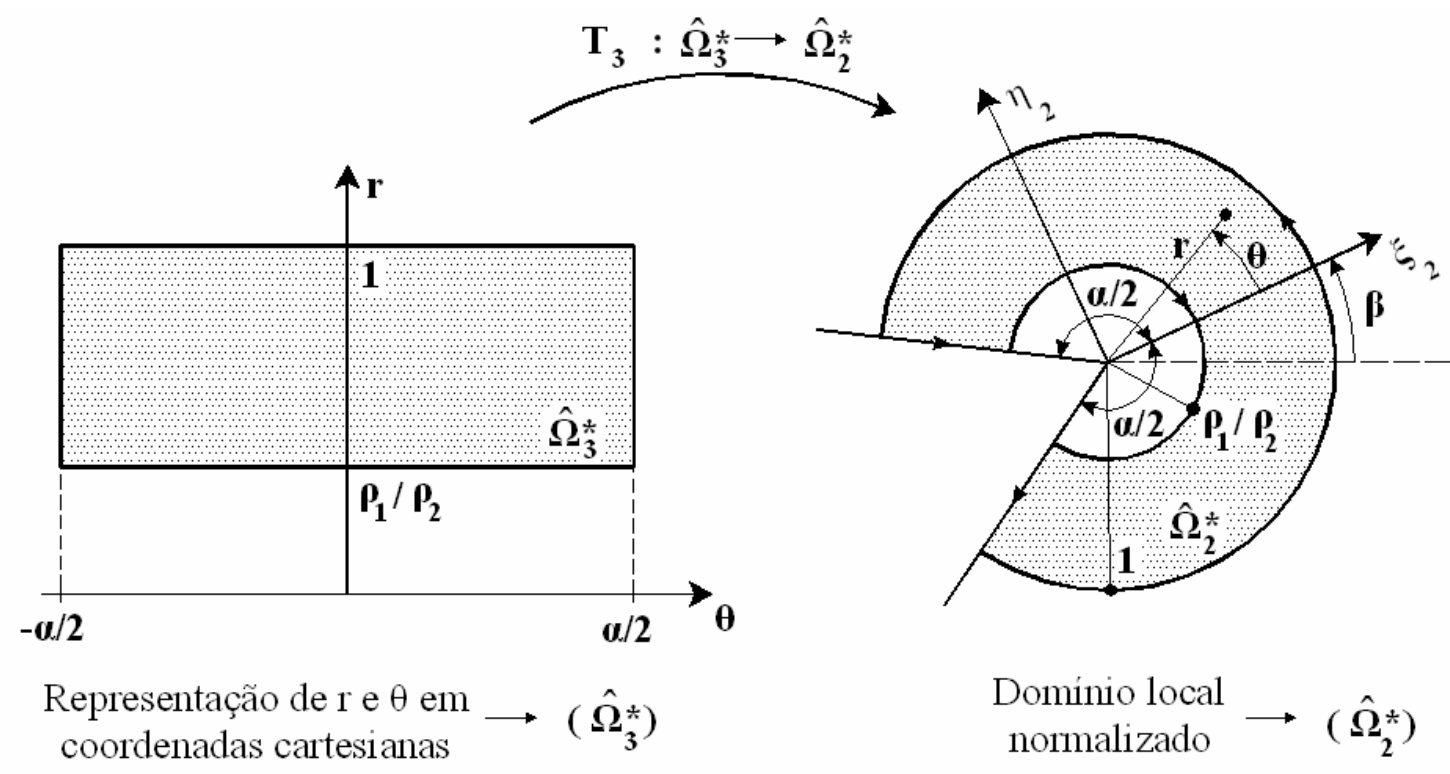

Figura 4.12 - Transformação de coordenadas para o método da função cutoff (MFC) Passo III.

O equacionamento para a transformação entre os espaços $\hat{\Omega}_{3}^{*}$ e $\hat{\Omega}_{2}^{*}$ é dado na forma:

$$
\begin{aligned}
& \mathrm{T}_{3}: \hat{\Omega}_{3}^{*} \rightarrow \hat{\Omega}_{2}^{*} \\
& \left(\begin{array}{l}
\xi_{2} \\
\eta_{2}
\end{array}\right)=\mathrm{r}\left(\begin{array}{c}
\cos (\theta) \\
\operatorname{sen}(\theta)
\end{array}\right) .
\end{aligned}
$$

O jacobiano para a transformação $T_{3}$ é dado por:

$$
\mathrm{J}_{3}=\left|\begin{array}{cc}
\frac{\partial \xi_{2}}{\partial \mathrm{r}} & \frac{\partial \eta_{2}}{\partial \theta} \\
\frac{\partial \eta_{2}}{\partial \mathrm{r}} & \frac{\partial \eta_{2}}{\partial \theta}
\end{array}\right|=\left|\begin{array}{cc}
\cos (\theta) & -\mathrm{rsen}(\theta) \\
\operatorname{sen}(\theta) & \mathrm{r} \cos (\theta)
\end{array}\right|=\mathrm{r}
$$

\section{$\underline{\text { Passo IV }}$}

Transformar as coordenadas do domínio de integração Gaussiana $\hat{\Omega}_{4}^{*}$ em coordenadas polares representadas em eixos cartesianos $\hat{\Omega}_{3}^{*}$ (Figura 4.13). 


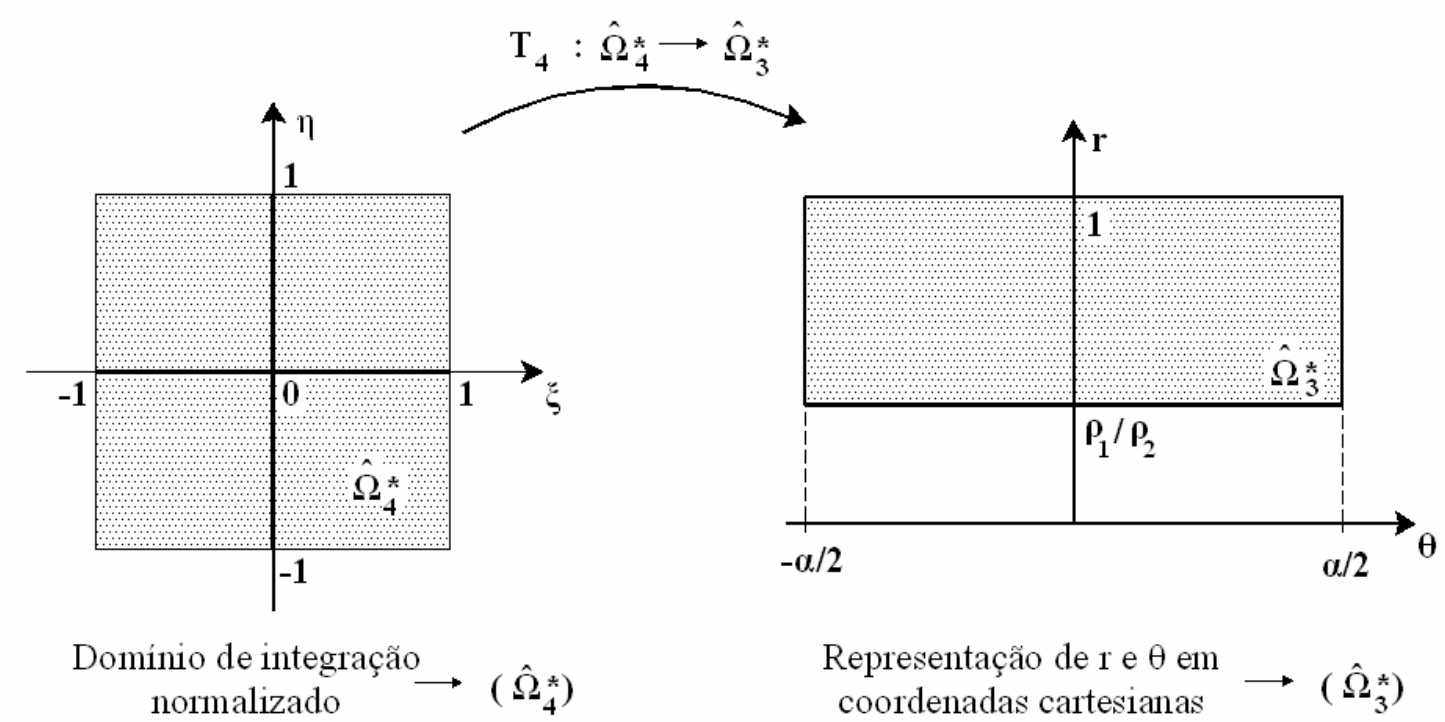

Figura 4.13 - Transformação de coordenadas para o método da função cutoff (MFC) Passo IV.

O equacionamento para essa transformação é:

$$
\begin{aligned}
& \mathrm{T}_{4}: \hat{\Omega}_{4}^{*} \rightarrow \hat{\Omega}_{3}^{*} \\
& \left(\begin{array}{l}
\mathrm{r} \\
\theta
\end{array}\right)=\left[\begin{array}{cc}
\frac{\alpha}{2} & 0 \\
0 & \frac{\left(\rho_{2}-\rho_{1}\right)}{2 \rho_{2}}
\end{array}\right]\left(\begin{array}{l}
\xi \\
\eta
\end{array}\right)+\left(\begin{array}{c}
0 \\
\frac{\left(\rho_{2}+\rho_{1}\right)}{2 \rho_{2}}
\end{array}\right) .
\end{aligned}
$$

O jacobiano para a transformação é dado por:

$$
\mathrm{J}_{4}=\left|\begin{array}{ll}
\frac{\partial \mathrm{r}}{\partial \xi} & \frac{\partial \mathrm{r}}{\partial \eta} \\
\frac{\partial \theta}{\partial \xi} & \frac{\partial \theta}{\partial \eta}
\end{array}\right|=\left|\begin{array}{cc}
\frac{\alpha}{2} & 0 \\
0 & \frac{\left(\rho_{2}-\rho_{1}\right)}{2 \rho_{2}}
\end{array}\right|=\frac{\alpha}{2} \frac{\left(\rho_{2}-\rho_{1}\right)}{2 \rho_{2}}
$$

\section{Equacionamento final}

O equacionamento final da transformação de coordenadas entre os espaços $\hat{\Omega}_{4}^{*} \mathrm{e}$ $\Omega^{*}$ é dado por: 


$$
\begin{aligned}
& T=T_{1} \circ T_{2} \circ T_{3} \circ T_{4} \\
& \left(\begin{array}{l}
X \\
Y
\end{array}\right)=\rho_{2}\left(\frac{\left(\rho_{2}-\rho_{1}\right)}{2 \rho_{2}} \eta+\frac{\left(\rho_{2}+\rho_{1}\right)}{2 \rho_{2}}\right)\left[\begin{array}{cc}
\cos (\beta) & -\operatorname{sen}(\beta) \\
\operatorname{sen}(\beta) & \cos (\beta)
\end{array}\right]\left(\begin{array}{c}
\cos \left(\frac{\alpha}{2} \xi\right) \\
\operatorname{sen}\left(\frac{\alpha}{2} \xi\right)
\end{array}\right)+\left(\begin{array}{l}
X_{0} \\
Y_{0}
\end{array}\right)
\end{aligned}
$$

O jacobiano total da transformação pode ser encontrado pelo produto dos jacobianos de cada transformação. Assim:

$$
\begin{aligned}
& J=J_{1} J_{2} J_{3} J_{4} \\
& J(\eta)=\left(\rho_{2}\right)^{2}\left[\left(\frac{\left(\rho_{2}-\rho_{1}\right)}{2 \rho_{2}} \eta+\frac{\left(\rho_{2}+\rho_{1}\right)}{2 \rho_{2}}\right)\right] \frac{\alpha}{2} \frac{\left(\rho_{2}-\rho_{1}\right)}{2 \rho_{2}} .
\end{aligned}
$$

Para as integrais de contorno nas arestas do vértice reentrante (3.38), o jacobiano pode ser escrito da seguinte forma:

$$
\mathrm{ds}=\sqrt{\left(\frac{\mathrm{dX}( \pm 1, \eta)}{\mathrm{d} \eta}\right)^{2}+\left(\frac{\mathrm{dY}( \pm 1, \eta)}{\mathrm{d} \eta}\right)^{2}} \mathrm{~d} \eta \rightarrow \mathrm{ds}=\frac{\left(\rho_{2}-\rho_{1}\right)}{2} \mathrm{~d} \eta
$$

$\xi=1$ indica a aresta $\Gamma_{3}^{*}$ e $\xi=-1$ indica a aresta $\Gamma_{4}^{*}$ (Figura 3.1).

\subsubsection{Formulação numérica}

Utilizando-se as coordenadas normalizadas de integração, a formulação para o método da função cutoff é dada por:

$$
\begin{aligned}
& \mathrm{A}_{\mathrm{j}}^{(\mathrm{m})}= \\
& \quad \int_{-1}^{1}\left\{\mathrm{u}_{\mathrm{EFG}}(\mathrm{X}(-1, \eta), \mathrm{Y}(-1, \eta))\right\}^{\mathrm{T}}\left\{\mathrm{T}^{\left(\mathrm{w}_{-j}^{(\mathrm{m})}\right)}(\mathrm{X}(-1, \eta), \mathrm{Y}(-1, \eta))\right\} \frac{\left(\rho_{2}-\rho_{1}\right)}{2} \mathrm{~d} \xi \\
& \quad-\int_{-1}^{1}\left\{\mathrm{u}_{\mathrm{EFG}}(\mathrm{X}(1, \eta), \mathrm{Y}(1, \eta))\right\}^{\mathrm{T}}\left\{\mathrm{T}^{\left(\mathrm{w}_{-j}^{(\mathrm{m})}\right)}(\mathrm{X}(1, \eta), \mathrm{Y}(1, \eta))\right\} \frac{\left(\rho_{2}-\rho_{1}\right)}{2} \mathrm{~d} \xi \\
& +\int_{-1-1}^{1} \int_{1}^{1}\left[\left(\frac{\partial \sigma_{x}^{\left(w_{-j}^{(m)}\right)}}{\partial \mathrm{x}}(\xi, \eta)+\frac{\partial \tau_{x y}^{\left(w_{-j}^{(m)}\right)}}{\partial y}(\xi, \eta)\right) \mathrm{u}_{\mathrm{EFG}}(\xi, \eta)\right] \mathrm{J} \mid \mathrm{d} \xi \mathrm{d} \eta \\
& \quad+\int_{-1-1}^{1} \int_{-1}^{1}\left[\left(\frac{\partial \tau_{x y}^{\left(w_{-j}^{(m)}\right)}}{\partial x}(\xi, \eta)+\frac{\partial \sigma_{y}^{\left(w_{-j}^{(m)}\right)}}{\partial y}(\xi, \eta)\right) \mathrm{u}_{\mathrm{EFG}}(\xi, \eta)\right]|\mathrm{J}| \mathrm{d} \xi \mathrm{d} \eta
\end{aligned}
$$


$\mathrm{u}_{\mathrm{EFG}}$ indica o campo de deslocamento solução do método de elementos finitos generalizados.

Simplificando e aplicando a integração Gaussiana, tem-se:

$$
\begin{aligned}
& A_{j}^{(m)}=\sum_{i=1}^{n p d^{(\eta)}}\left(\left\{w_{-j}^{(m)}\left(-1, \eta_{i}\right)\right\}^{T}\left\{T^{\left(u_{E F G}\right)}\left(-1, \eta_{i}\right)\right\}-\left\{u_{E F G}\left(-1, \eta_{i}\right)\right\}^{T}\left\{T^{\left(w_{-j}^{(m)}\right)}\left(-1, \eta_{i}\right)\right\}\right) \omega_{1}^{(\eta)} \frac{\left(\rho_{2}-\rho_{1}\right)}{2} \\
& -\sum_{\mathrm{i}=1}^{\mathrm{npG}}\left(\left\{\mathrm{w}_{-\mathrm{j}}^{(\mathrm{m})}\left(-1, \eta_{\mathrm{i}}\right)\right\}^{\mathrm{T}}\left\{\mathrm{T}^{\left(\mathrm{u}_{\mathrm{EFG}}\right)}\left(-1, \eta_{\mathrm{i}}\right)\right\}-\left\{\mathrm{u}_{\mathrm{EFG}}\left(-1, \eta_{\mathrm{i}}\right)\right\}^{\mathrm{T}}\left\{\mathrm{T}^{\left(\mathrm{w}_{-\mathrm{j}}^{(\mathrm{m})}\right)}\left(-1, \eta_{\mathrm{i}}\right)\right\}\right) \omega_{1}^{(\eta)} \frac{\left(\rho_{2}-\rho_{1}\right)}{2}
\end{aligned}
$$

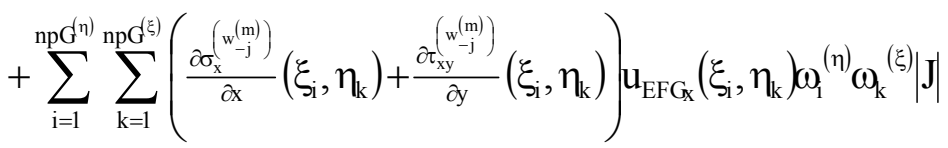

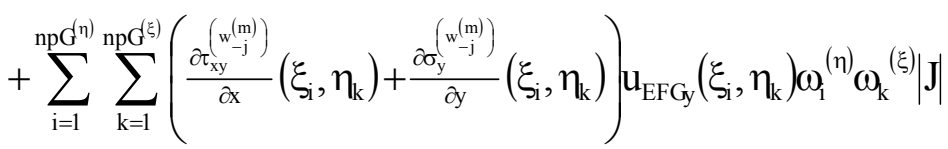

sendo $\mathrm{npG}^{(\xi)}$ o número de pontos de Gauss na direção $\xi$ e $\mathrm{npG}^{(\eta)}$ o número de pontos de Gauss na direção $\eta$. 


\section{Aspectos computacionais}

A solução numérica utilizada na extração dos fatores de intensidade de tensão é obtida por meio do programa Scientific and Engineering Toolkit (SET). Esse programa, desenvolvido pelo orientador do presente trabalho, utiliza recursos de programação orientada a objetos, em $\mathrm{C}++$ para Linux, que facilitam a reutilização do código.

Neste capítulo, apresentam-se uma breve descrição do programa SET e uma descrição dos principais recursos computacionais utilizados no desenvolvimento da implementação dos métodos de extração.

\subsection{O paradigma da Programação Orientada a Objetos (POO)}

A programação orientada a objetos (POO) (DEITEL; DEITEL, 2001; LIPPMAN e LAJOIE, 1998; YAROSHENKO, 1994) é um estilo de programação que combina dados (variáveis) e as funções (métodos) que são utilizadas na manipulação desses dados em uma só entidade chamada de objeto. Esse estilo de programação foi desenvolvido com o objetivo de suplantar diversas limitações encontradas em linguagens de programação convencionais, como por exemplo, as linguagens de programação estruturadas. A POO conservou todas as boas idéias da programação estruturada e acrescentou novos aspectos que permitem uma nova abordagem de programação.

A POO não foi desenvolvida com a intenção de substituir a programação estruturada tradicional. Esse novo conceito de programação propõe a evolução das práticas recomendadas em programação estruturada. O Quadro 5.1 apresenta uma comparação entre as principais características da POO e da programação estruturada. 
Quadro 5.1 - Características da programação orientada a objetos e da programação estruturada.

\begin{tabular}{|c|c|}
\hline Programação Orientada a Objetos & Programação Estruturada \\
\hline Métodos & Procedimentos e funções \\
\hline Instâncias de variáveis & Variáveis \\
\hline Mensagens & Chamadas e procedimentos \\
\hline Classes & Tipos de dados definidos pelo usuário \\
\hline Herança & - \\
\hline Polimorfismo & - \\
\hline
\end{tabular}

A idéia principal da POO propõe que o projeto do programa deve ser desenvolvido a partir de entidades do mundo real (objetos) e não a partir de dados e procedimentos como é feito em programação estruturada. A programação estruturada tem como principal foco as ações (procedimentos e funções), a POO enfatiza a manipulação de objetos e suas interações.

O principal objetivo da POO é reduzir a complexidade no desenvolvimento de um software e aumentar sua produtividade. Tais objetivos promovem duas características importantes da POO: a reutilização do código e a redução no tempo de manutenção e desenvolvimento de programas.

A reutilização efetiva do código é assegurada por um conceito importante da POO, o conceito de classe. Denomina-se classe as variáveis e métodos que representam características de um conjunto de objetos.

Há três idéias básicas em programação orientada a objetos: encapsulamento, herança e polimorfismo. Tais idéias são descritas a seguir.

\subsubsection{Encapsulamento}

O conceito de encapsulamento está relacionado com a ação de ocultar informações (dados e códigos). O encapsulamento funciona como uma proteção para as variáveis e métodos, além de tornar explícito qualquer tipo de comunicação com o objeto.

Umas das vantagens de se trabalhar com classes é o encapsulamento das variáveis e métodos. Os dados e funções que atuam sobre os mesmo objetos encontramse agrupados em uma mesma entidade, permitindo que se tenha uma melhor definição 
do escopo dos procedimentos e uma maior segurança no acesso aos dados. Em uma linguagem de programação convencional, os dados são declarados de forma independente das funções.

\subsubsection{Herança}

O mecanismo de herança permite definir uma nova classe, com base em outra classe já existente. A classe criada, denominada de subclasse ou classe derivada, automaticamente herda todas as variáveis e métodos existentes na classe base (superclasse). O mecanismo de herança permite ainda que a subclasse inclua ou sobreponha novas variáveis e métodos da superclasse.

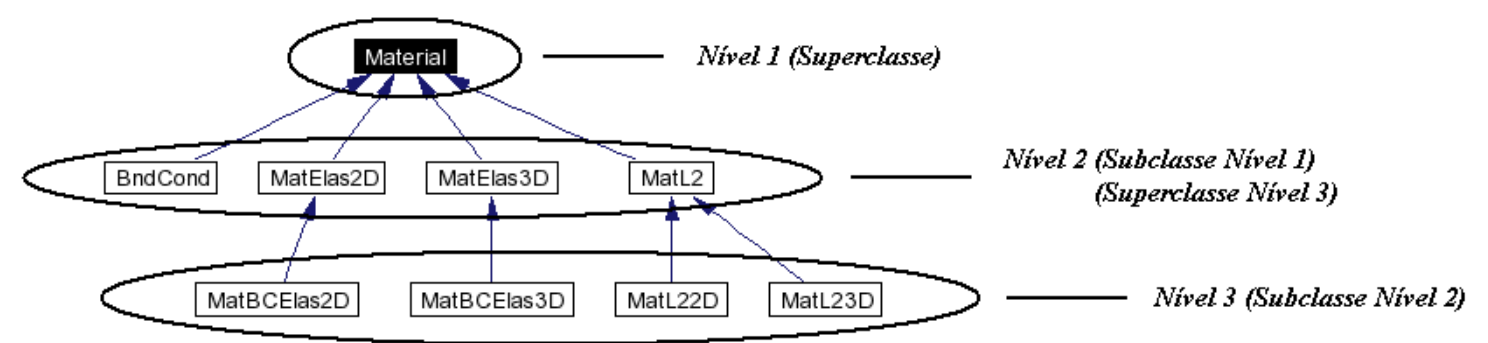

Figura 5.1 - Exemplo de hierarquia de classes.

O mecanismo de herança é recursivo, ou seja, permite criar uma hierarquia de classes. Nos níveis mais altos da hierarquia estão as características comuns a todos os objetos desta classe, enquanto que nos níveis inferiores estão particularizações das classes superiores.

Há dois tipos básicos de implementação de herança: simples e múltipla. $\mathrm{Na}$ herança simples, a subclasse herda variáveis e métodos de apenas uma classe e na herança múltipla a subclasse herda variáveis e métodos de diversas classes.

O mecanismo de herança pode ser aplicado no uso de bibliotecas de classes. Essa prática permite uma capacidade maior de compartilhamento e reutilização de código. Dessa forma, é possível criar subclasses para atender novas necessidades do programa, em função de classes já existentes. No programa SET, utilizam-se algumas bibliotecas como $C++$ Standard library, $C++$ BOOST Libraries (C++ BOOST, 2004) e Scientific and Engineering Programming in $C++$ (BARTON; NACKMAN, 1994) no desenvolvimento das classes pertencentes ao programa. 


\subsubsection{Polimorfismo}

O mecanismo de polimorfismo permite o compartilhamento de um método nos níveis acima e abaixo na hierarquia de classes, com cada classe na hierarquia implementando o método compartilhado de forma apropriada para o seu nível hierárquico.

O polimorfismo permite tratar objetos semelhantes de uma maneira uniforme. Esse mecanismo da POO permite que se execute um mesmo método em diversos níveis da hierarquia de classe e o objeto de cada nível responda de uma forma diferente, apropriada para o nível ao qual ele pertence.

O polimorfismo exige a utilização do conceito de herança para ser implementado e aplica-se apenas aos métodos (funções ou procedimentos) da classe.

\subsection{O programa SET (Scientific and Engineering Toolkit)}

O Programa SET implementa o método de elementos finitos generalizados (MEFG) e alguns de seus objetos são utilizados no código desenvolvido para os métodos de extração. A seguir, apresenta-se uma breve descrição das principais classes implementadas no programa SET. Vale salientar que o projeto de várias classes utilizadas no SET foi influenciado pelo trabalho pioneiro em POO de Devloo (1992), autor do sistema PZ (BRAVOS, 2004).

Nas próximas seções, apresentam-se os recursos da POO na implementação das classes do programa SET na forma de diagramas. Em todos os diagramas utiliza-se a legenda descrita no Quadro 5.2. 
Quadro 5.2 - Legenda da representação gráfica para as classes.

\begin{tabular}{|c|c|l|}
\hline $\mathbf{N}$ & $\begin{array}{c}\text { Representação } \\
\text { gráfica }\end{array}$ & \multicolumn{1}{|c|}{ Descrição } \\
\hline $\mathbf{1}$ & Nome da Classe & Representa a classe para a qual o diagrama foi gerado. \\
\hline $\mathbf{2}$ & Nome da Classe & $\begin{array}{l}\text { Representa uma subclasse da classe que aparece na forma da } \\
\text { representação } 1 \text { ou uma classe que colabora em seu } \\
\text { desenvolvimento. }\end{array}$ \\
\hline $\mathbf{3}$ & $-\begin{array}{l}\text { Utilizada para representar a relação de herança entre as } \\
\text { classes. }\end{array}$ \\
\hline $\mathbf{4}$ & - Nome do objeto & $\begin{array}{l}\text { Utilizada para representar que uma classe está contida em } \\
\text { outra ou é utilizada por outra. O nome do objeto que é } \\
\text { utilizado para relacionar as classes segue ao longo da seta. }\end{array}$ \\
\hline
\end{tabular}

\subsubsection{Classe geometria do elemento (GeoEl)}

A classe GeoEl implementa as características geométricas dos elementos. Dentre as características geométricas do elemento citam-se: os jacobianos das transformações diretas e inversas, os vetores normais e tangentes aos elementos, etc. A Figura 5.2 apresenta a hierarquia da classe GeoEl.

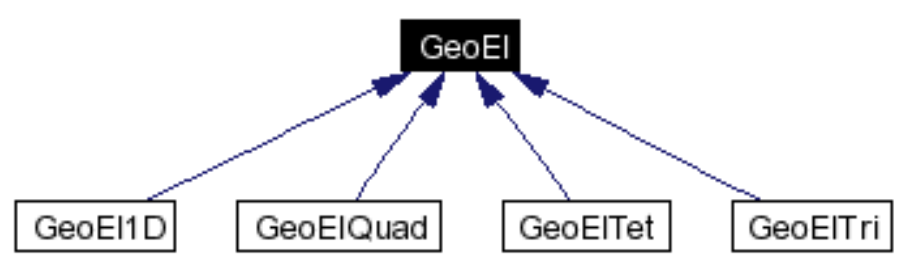

Figura 5.2 - Representação gráfica da hierarquia da classe GeoEl.

As subclasses da classe GeoEl implementam as funções de forma lineares e criam uma base de dados para aplicação das condições de contorno para cada tipo de elemento que possui implementação desenvolvida no programa SET. Os elementos e as subclasses são associados da seguinte forma:

- GeoEl1D: elemento unidimensional;

- GeoElQuad: elemento quadrilateral; 
- GeoElTri: elemento triangular;

- GeoElTet: elemento tetraédrico.

A Figura 5.3 apresenta um diagrama que ilustra a forma com que outras classes do programa SET auxiliam no desenvolvimento da classe GeoEl.

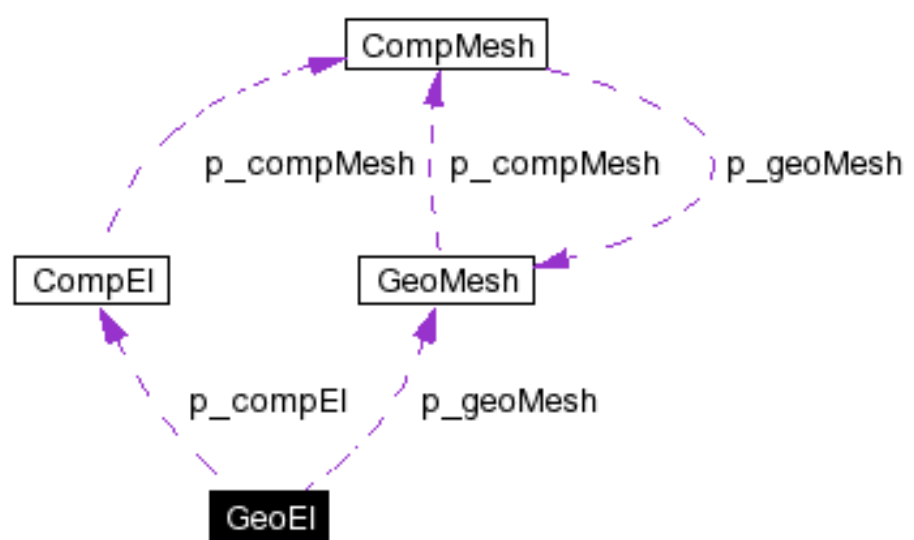

Figura 5.3 - Representação gráfica das classes que colaboram no desenvolvimento da classe GeoEl.

As classes CompEl, CompMesh e GeoMesh são classes implementadas no programa SET e $p \_c o m p E l, p \_c o m p M e s h$ e $p \_g e o M e s h$ são ponteiros (tipo de variável que armazena o endereço de memória para outras variáveis e objetos) para objetos dessas classes (Figura 5.3). Uma breve descrição dessas classes será apresentada nas próximas seções.

\subsubsection{Classe elemento computacional (CompEl)}

A classe CompEl implementa as características computacionais dos elementos implementados no programa SET. Como exemplos citam-se: o cálculo da matriz de rigidez, o cálculo das condições de contorno de Neumman aplicadas ao elemento, etc. A Figura 5.4 ilustra a representação gráfica da hierarquia da classe CompEl. 


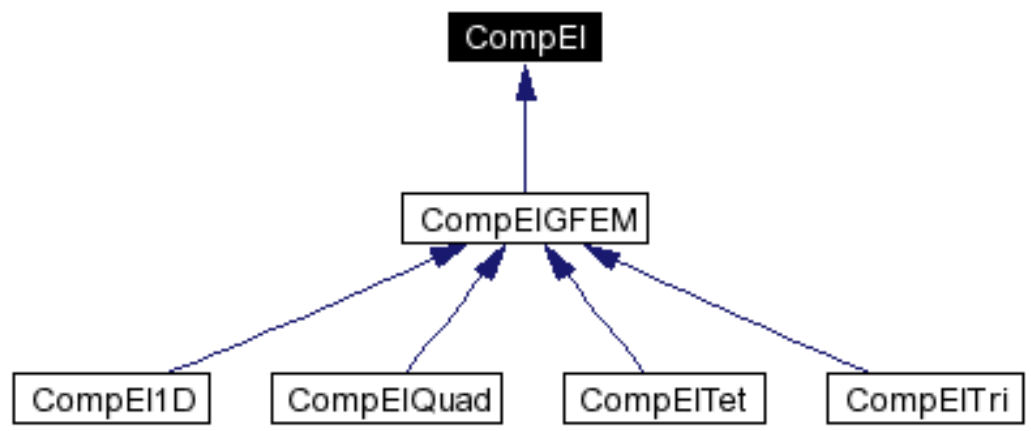

Figura 5.4 - Representação gráfica da hierarquia da classe CompEl.

Nas subclasses da classe CompElGFEM estão implementadas as funções de forma de elementos finitos generalizados e suas respectivas derivadas para cada elemento finito implementado no programa SET. As subclasses da classe CompElGFEM estão relacionadas com os elementos da seguinte forma:

- CompEl1D: elemento unidimensional;

- CompElQuad: elemento quadrilateral;

- CompElTri: elemento triangular;

- CompElTet: elemento tetraédrico.

A Figura 5.5 apresenta um diagrama que ilustra a forma com que outras classes do programa SET colaboram no desenvolvimento da classe CompEl.

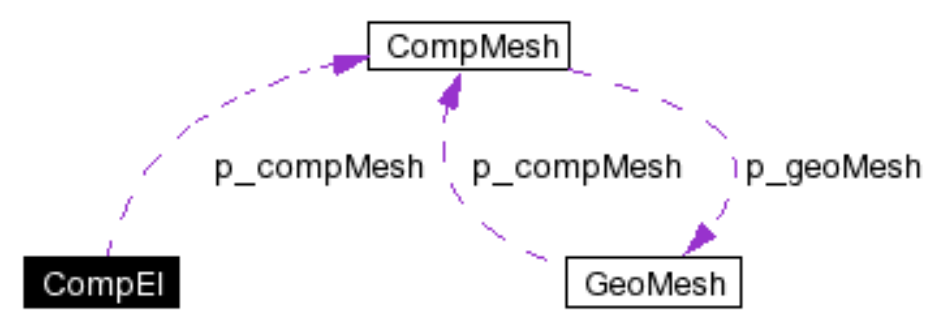

Figura 5.5 - Representação gráfica das classes que auxiliam no desenvolvimento da classe CompEl.

CompMesh e GeoMesh são classes implementadas no programa SET e p_compMesh e $p \_g e o M e s h$ são ponteiros para objetos dessas classes (Figura 5.5). Uma breve descrição das classes CompMesh e GeoMesh será apresentada nas próximas seções. 


\subsubsection{Classe nó geométrico (GeoNod)}

Armazena as características geométricas dos nós do elemento como, por exemplo, coordenadas globais do nó e identificadores numéricos do nó.

\subsubsection{Classe nó computacional (CompNod)}

A classe CompNod está associada às características computacionais que um nó em uma malha de elementos finitos generalizados possui. Como exemplos de características computacionais citam-se: as funções de forma de alta ordem polinomial, associadas ao nó em coordenadas globais, utilizadas para o enriquecimento da solução em uma determinada direção, e suas primeiras derivadas.

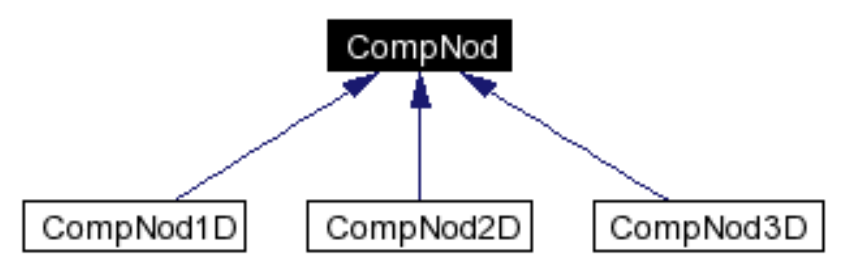

Figura 5.6 - Representação gráfica da hierarquia da classe CompNod.

As subclasses CompNod1D, ComNod2D e CompNod3D estão associadas aos nós de elementos unidimensionais, bidimensionais e tridimensionais respectivamente.

A Figura 5.7 apresenta um diagrama com as classes que auxiliam no desenvolvimento da classe CompNod.

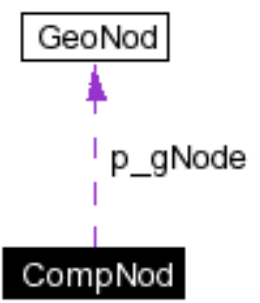

Figura 5.7 - Representação gráfica das classes que colaboram no desenvolvimento da classe CompNod.

A classe CompNod obtém diretamente as informações necessárias sobre o nó utilizando objetos da classe GeoNod. $p \_$gNode é um ponteiro para um objeto da classe GeoNod. 


\subsubsection{Classe malha geométrica (GeoMesh)}

A classe GeoMesh implementa as características geométricas da discretização utilizada na modelagem do problema. Um dos principais métodos implementados nessa classe é a função que, a partir da informação de um ponto qualquer do domínio, informa se o ponto está dentro do domínio de análise e a qual elemento esse ponto pertence. A classe GeoMesh também é utilizada para armazenar as coordenadas globais e índices dos nós e elementos da discretização do problema.

A Figura 5.8 apresenta um diagrama que ilustra a forma com que outras classes colaboram no desenvolvimento da classe GeoMesh.

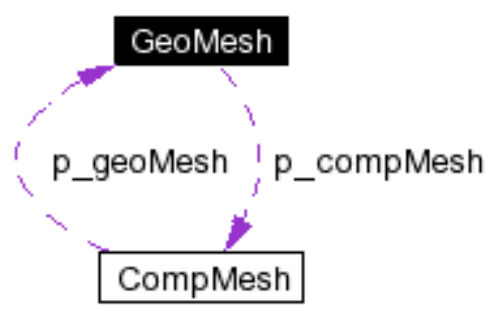

Figura 5.8 - Representação gráfica das classes que auxiliam no desenvolvimento da classe GeoMesh.

\subsubsection{Classe malha computacional (CompMesh)}

A classe CompMesh implementa as características computacionais da discretização utilizada na modelagem do problema. Os principais métodos implementados nessa classe são: as funções que lêem e armazenam todas as informações existentes nos nós e elementos computacionais (CompNod e GeoNod) e a função responsável pela montagem da matriz global do problema.

A Figura 5.8 apresenta um diagrama que ilustra a forma com que outras classes auxiliam no desenvolvimento da classe CompMesh.

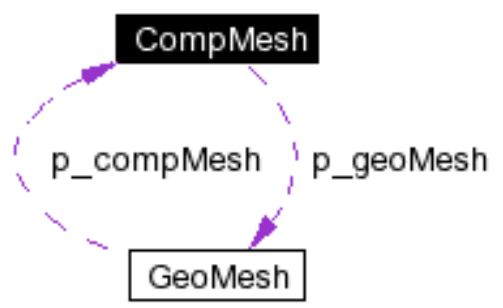

Figura 5.9 - Representação gráfica das classes que colaboram no desenvolvimento da classe CompMesh. 


\subsubsection{Classe material ou equação diferencial (Material)}

O elemento computacional (objeto da classe CompEl) calcula as funções de forma e suas derivadas e integra as funções de forma sobre o elemento geométrico (objeto da classe $G e o E l$ ). O objeto da classe CompEl ignora o tipo de problema que está sendo analisado. O cálculo dos coeficientes da equação diferencial discretizada ou forma bilinear é de responsabilidade exclusiva da classe Material. A classe Material define a equação diferencial a ser discretizada. Essa classe tem como finalidade calcular a contribuição do elemento para a matriz de rigidez, o vetor de forças externas, as condições de contorno e o trabalho das forças externas no ponto de integração. A Figura 5.10 ilustra a hierarquia da classe Material.

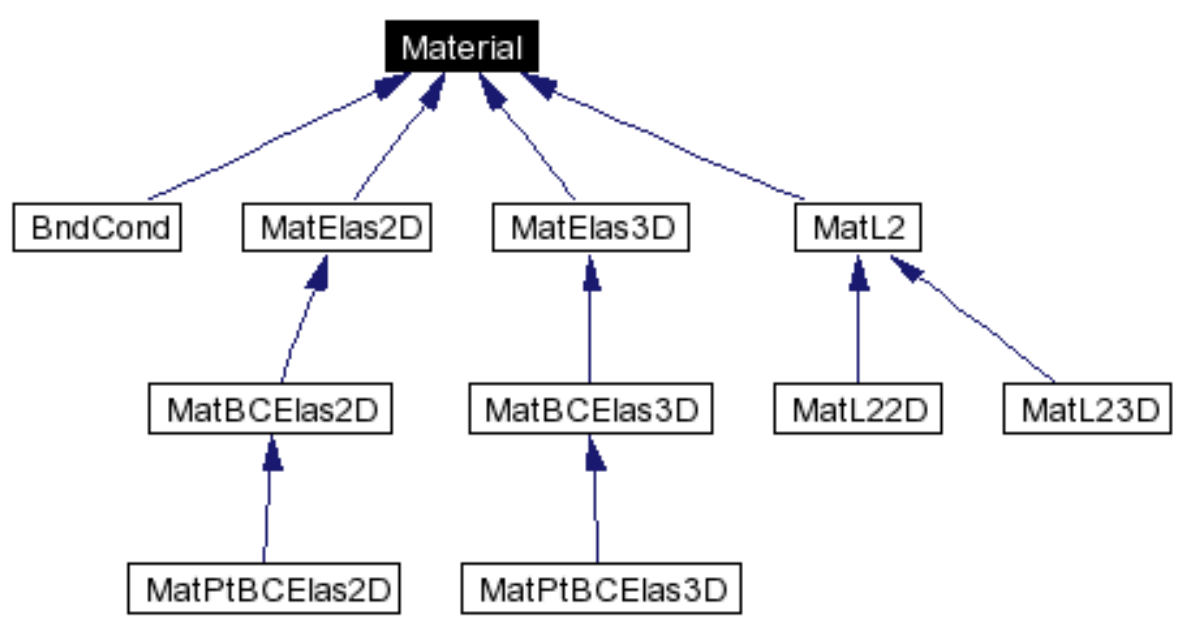

Figura 5.10 - Representação gráfica da hierarquia da classe Material.

A classe Material serve como base para a definição dos tipos de análise que podem ser desenvolvidas no programa SET. O programa SET implementa os problemas de elasticidade bidimensional e tridimensional. Outros tipos de análise podem ser implementados apenas inserindo mais uma subclasse na hierarquia da classe material, sem alterar outras classes do programa.

\subsubsection{Classe SpecialBasis}

A classe SpecialBasis é uma classe base que define o comportamento de classes usadas para construir funções de forma especiais para o enriquecimento da solução do MEFG como, por exemplo, a expansão assintótica próximo a ponta da fissura e outras soluções analíticas. Essa classe pode ser utilizada, por meio de objetos, por outras 
classes como, por exemplo, a classe CompNod. A hierarquia da classe SpecialBasis é ilustrada na Figura 5.11.

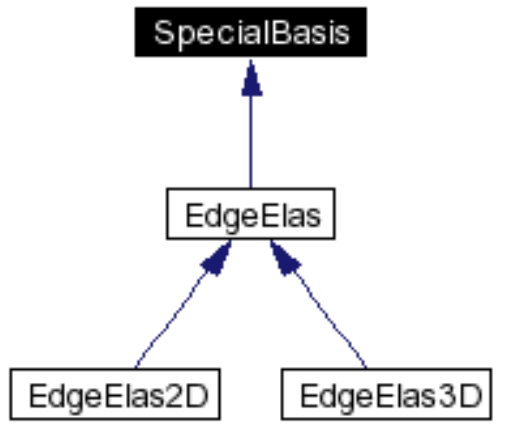

Figura 5.11 - Representação gráfica da hierarquia da classe SpecialBasis.

As subclasses EdgeElas2D e EdgeElas3D implementam a expansão assintótica para regiões reentrantes no domínio em problemas elásticos bidimensionais e tridimensionais, respectivamente.

As soluções implementadas na classe SpecialBasis são utilizadas por outras classes. A classe ExactSol tem como propósito a implementação de soluções exatas para serem utilizadas em problemas clássicos da mecânica como, por exemplo, aplicar as tensões da solução exata na aresta do domínio de um painel fraturado (subclasse EdgeSol2D). A Figura 5.12 ilustra a hierarquia da classe ExactSol.

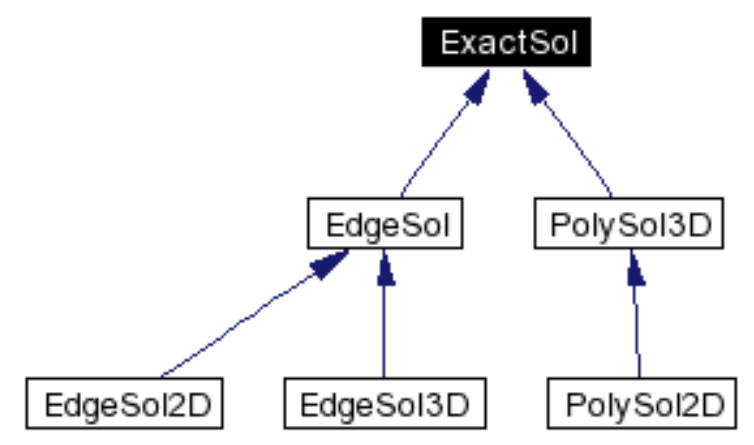

Figura 5.12 - Representação gráfica da hierarquia da classe ExactSol.

A Figura 5.13 ilustra graficamente a interação existente entre as classes ExactSol e SpecialBasis no desenvolvimento da subclasse EdgeSol. 


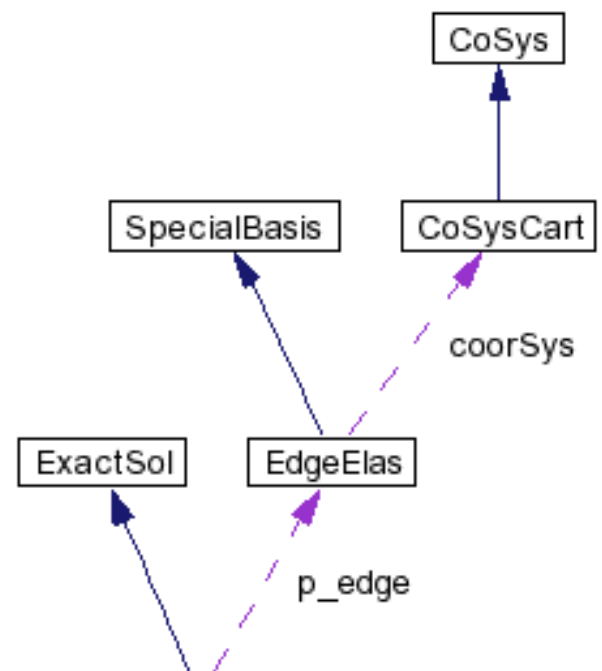

EdgeSol

Figura 5.13 - Representação gráfica das classes que colaboram no desenvolvimento da classe EdgeSol.

\subsubsection{Classe CoSys}

Nesta classe, estão implementadas as transformações entre coordenadas locais e globais. A subclasse CoSysCart implementa as transformações para coordenadas cartesianas entre os domínios dos elementos (domínio local) e o domínio de análise (domínio global). A classe CoSysCart aplica translações e rotações entre sistemas de coordenadas cartesianas e pode ser utilizada, não só para relacionar sistemas de coordenadas no elemento e coordenadas global, como também para relacionar sistemas de coordenadas cartesianas em outras classes do programa. A Figura 5.14 ilustra a hierarquia da classe CoSys.

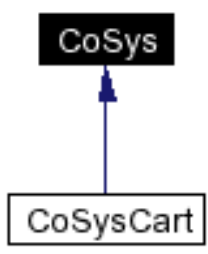

Figura 5.14 - Representação gráfica da hierarquia da classe CoSys.

\subsubsection{Classes para integração numérica (IntPoints e IntRuleList)}

A classe IntPoints é uma classe base para todas as classes que utilizam regras de integração numérica. A Figura 5.15 representa graficamente a hierarquia da classe 
IntPoints. A principal função dessa hierarquia de classes é fornecer os pontos de integração para as integrais dos elementos.

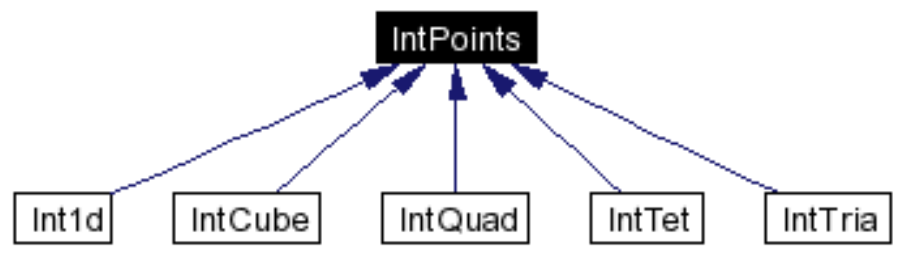

Figura 5.15 - Representação gráfica da hierarquia da classe IntPoints.

As classes derivadas de IntPoints implementam as regras de integração para alguns tipos de elementos finitos. As relações entre as classes derivadas e os elementos são:

- Int1D: elemento unidimensional;

- IntTri: elemento triangular;

- IntQuad: elemento quadrilateral;

- IntTet: elemento tetraédrico;

- IntCube: elemento cúbico.

As regras de integração utilizadas para os elementos são gerenciadas pela classe IntRuleList. A representação gráfica da classe IntRuleList e de suas classes auxiliares é apresentada na Figura 5.16.

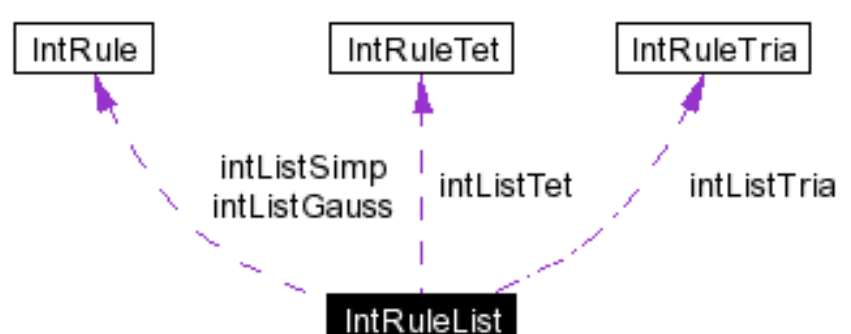

Figura 5.16 - Representação gráfica da classe IntRuleList e de suas classes auxiliares.

As classes auxiliares da classe IntRuleList e suas funções específicas são descritas da seguinte forma: 
- Classe IntRule: implementa regra de integração quadratura Gaussiana e é utilizada nas integrais dos elementos (CompEL) unidimensionais, quadrilaterais e cúbicos, essa classe também implementa a regra de integração de Simpson;

- Classe IntRuleTri: implementa a regra de integração para elementos triangulares;

- Classe IntRuleTet: implementa a rega de integração para elementos tetraédricos.

\subsubsection{Classe para etapa de pré-processamento (DataFileI)}

A classe DataFileI organiza as funções de leitura e armazenamento dos dados de entrada para o problema que será analisado pelo programa SET. Um diagrama que ilustra a hierarquia dessa classe é apresentado na Figura 5.17.

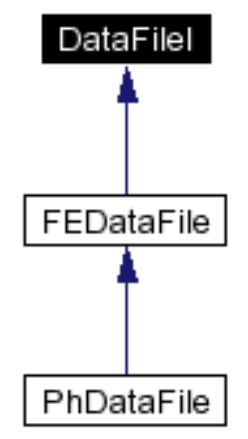

Figura 5.17 - Representação gráfica da hierarquia da classe DataFileI.

O código desenvolvido para a etapa de pré-processamento do programa SET lê os dados no formato Phlex (ALTAIR ENGINEERING, 2004). O arquivo de entrada de dados Phlex descreve o problema pela definição de nós, conectividade dos elementos, propriedades dos materiais, condições de contorno e alguns dados específicos do problema como o sistema de coordenadas na ponta da fissura. O arquivo de entrada é dividido em sete pacotes básicos de dados:

- DOMAIN: informa o tipo de domínio de análise (1D, 2D ou 3D);

- NODES: informa numeração e coordenadas dos nós;

- ELEMENTS: informa a conectividade dos elementos e o tipo de material associado;

- MATERIALS: informa as características dos materiais utilizados na análise;

- BOUDARY: descreve as condições de contorno; 
- BCASSIGN: associa as condições de contorno ao nó ou elemento;

- PROBLEMDATA: informa dados específicos do problema como, por exemplo, informações do sistema de coordenadas na ponta da fissura.

A implementação do código para a entrada de dados é bastante eficiente e possui uma complexidade linear, em virtude do uso extensivo das bibliotecas $C++$ Standard e C++ Boost Libraries (C++ BOOST, 2004).

\subsubsection{Classe para etapa de análise (Analysis)}

Nesta classe está implementada a etapa de análise do programa SET. A principal função dessa classe é a resolução do sistema de equações que representa o problema analisado com o método dos elementos finitos generalizados. Na classe Analysis também está implementada a função que utiliza a solução exata do problema como condição de contorno, fazendo o uso da classe ExactSol. A Figura 5.18 apresenta a hierarquia da classe Analysis.

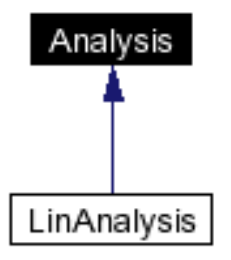

Figura 5.18 - Representação gráfica da hierarquia da classe Analysis.

A classe derivada LinAnalysis é uma especialização da classe Analysis para problemas lineares. Na implementação da classe Analysis utilizam-se outras classes do programa SET. O diagrama da Figura 5.19 ilustra o uso de outras classes no desenvolvimento da classe Analysis.

Utilizando as classes CompMesh e GeoMesh, a classe Analysis obtém as informações necessária para compor o sistema de equações gerado pela discretização do problema utilizando o Método de Elementos Finitos Generalizados (MEFG). 


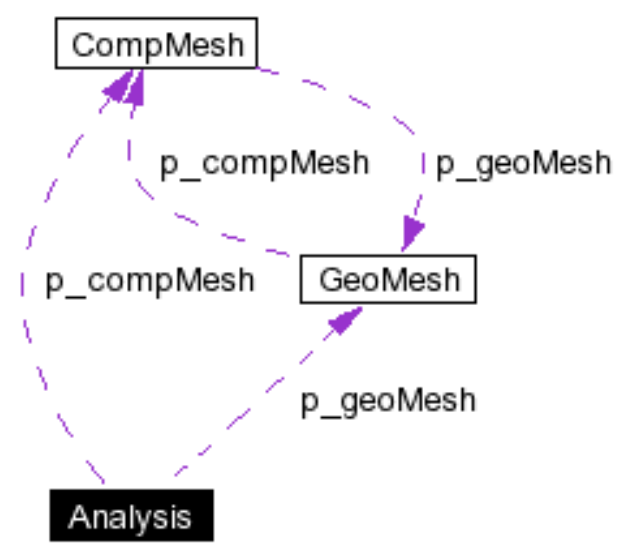

Figura 5.19 - Representação gráfica da colaboração de outras classes no desenvolvimento da classe Analysis.

\subsubsection{Classes para representação gráfica (GraphEl e GraphMesh)}

A classe GraphEl armazena as informações gráficas do elemento que serão posteriormente utilizadas na composição do arquivo de dados para saída gráfica. Essa classe gera uma discretização interna ao elemento para fins de representação gráfica. Cada elemento finito implementado no programa SET possui uma implementação distinta da classe GraphEl. A hierarquia da classe GraphEl é apresentada na Figura 5.20 .

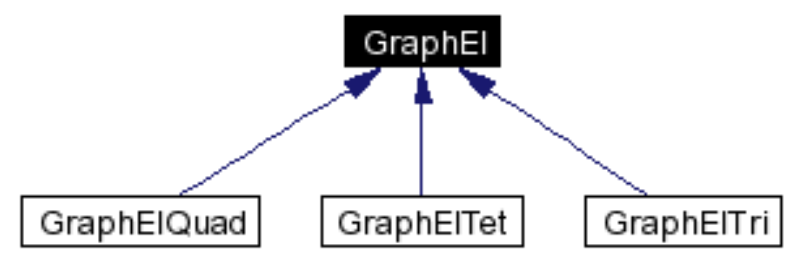

Figura 5.20 - Representação gráfica da hierarquia da classe GraphEl.

As relações entre as classes derivadas e os elementos são:

- GraphElQuad: elemento quadrilateral;

- GraphElTri: elemento triangular.

- GraphElTet: elemento tetraédrico;

A classe GraphEl interage com as classes GraphMesh, que armazena os dados da malha para visualização gráfica, e DataFileO, que gera um arquivo de 
saída para a visualização gráfica. Uma breve descrição das classes DataFileO e GraphMesh é apresentada na Seção 5.2.14. A Figura 5.21 ilustra graficamente a interação entre as classes GraphEl, DataFileO e GraphMesh.

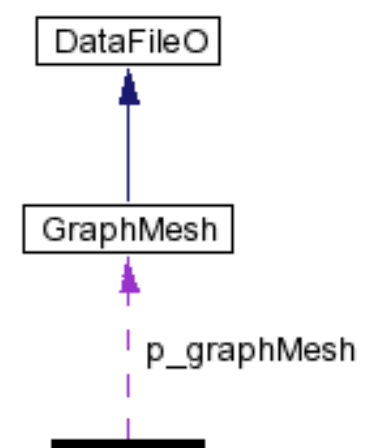

GraphEl

Figura 5.21 - Representação gráfica da colaboração de outras classes no desenvolvimento da classe GraphEl.

\subsubsection{Classe para etapa de pós-processamento (DataFileO)}

A classe DataFileO é responsável pela geração da saída de dados em arquivo, que será utilizado na representação gráfica, para o problema analisado no programa SET. Um diagrama que ilustra a hierarquia da classe é apresentado na Figura 5.22.

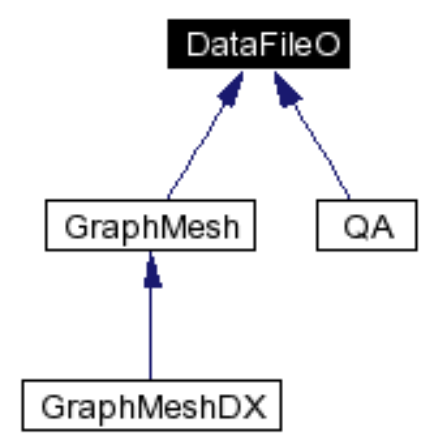

Figura 5.22 - Representação gráfica da hierarquia da classe DataFileO.

A saída de dados em arquivo para a representação gráfica do problema é gerada pela classe GraphMeshDX que, por sua vez, utiliza os dados armazenados na classe GraphMesh. O arquivo de saída gráfica é visualizado em uma interface gráfica (SET $D X$ ) desenvolvida no aplicativo OpenDX para Linux (Figura 5.23). O OpenDX é uma 
linguagem de programação visual desenvolvida para a manipulação gráfica de dados e criação de interfaces (OPENDX, 2004).

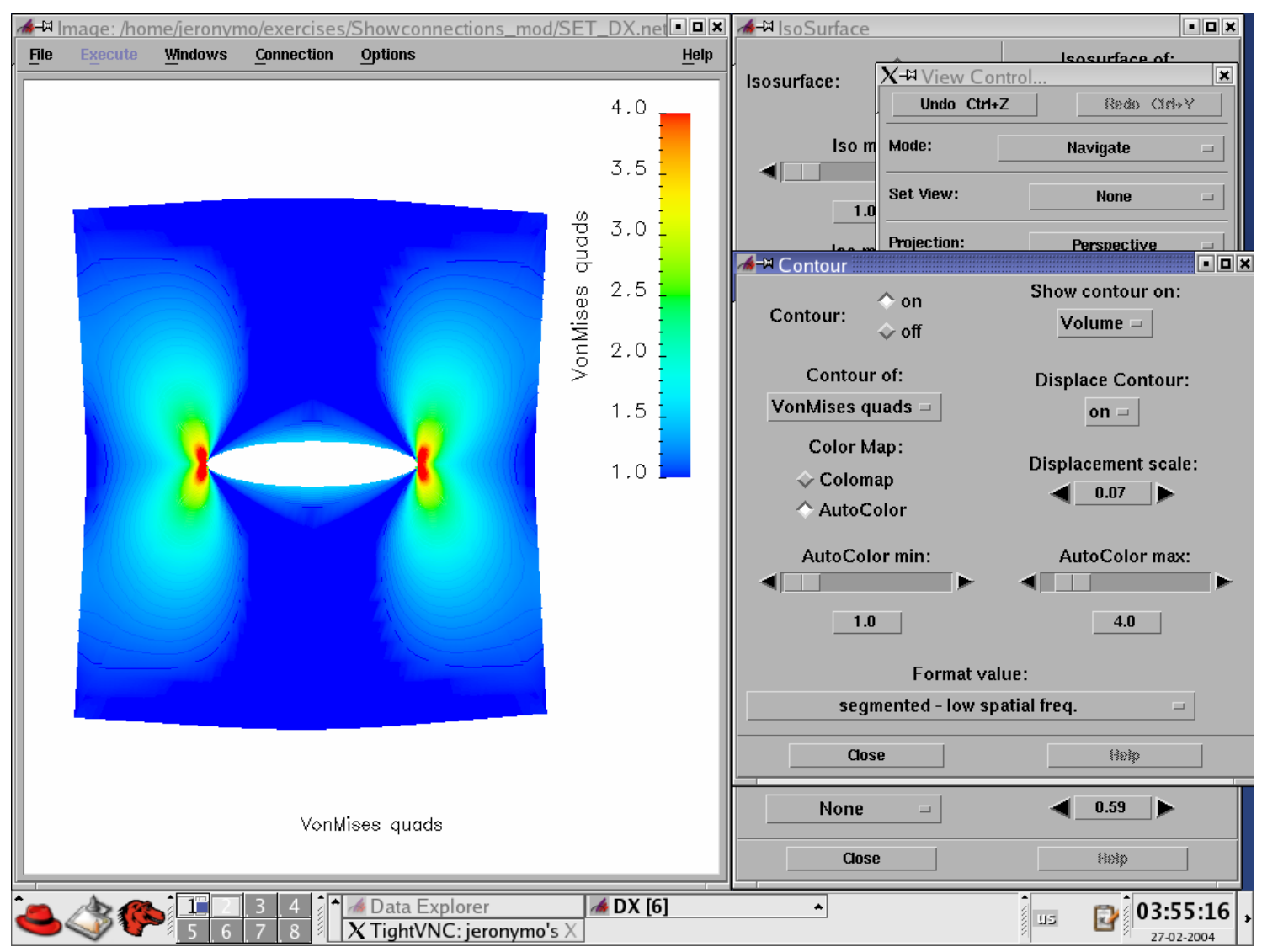

Figura 5.23 - Interface gráfica para visualização da saída de dados do programa SET

(SET DX).

\subsection{Implementação dos métodos de extração (ExtractSIF)}

Nesta seção, apresentam-se as classes desenvolvidas para a implementação dos métodos de extração proposta no presente trabalho. Ilustram-se os recursos computacionais utilizados na implementação dos métodos de extração MIC, MFC e Integral-J. Em virtude das características da Programação Orientada a Objetos (POO), diversas partes do código, já implementadas no programa SET, foram reutilizadas nas implementações das classes dos métodos de extração. A reutilização do código foi aplicada por meio de objetos das classes existentes no programa SET. A Figura 5.24 apresenta a hierarquia da classe ExtractSIF. A classe ExtractSIF serve como classe base para as implementações dos métodos de extração. 


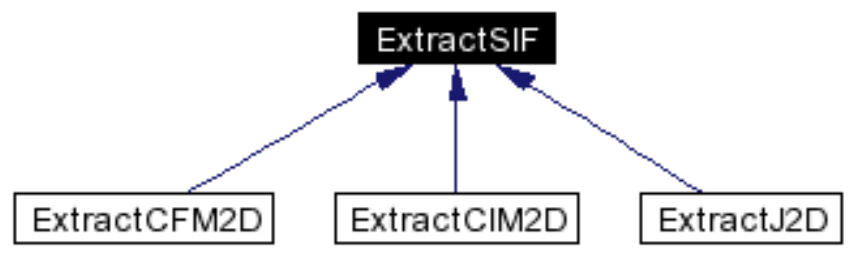

Figura 5.24 - Representação gráfica da hierarquia da classe ExtractSIF.

As classes derivadas da classe base ExtractSIF implementam os métodos de extração MIC, MFC e Integral-J.

- ExtractCFM2D: implementa o método de extração MFC para problemas bidimensionais;

- ExtractCIM2D: implementa o método de extração MIC para problemas bidimensionais;

- ExtractJ2D: implementa o método da Integral-J para problemas bidimensionais.

Uma breve descrição das classes do programa SET e dos recursos computacionais da POO, utilizados na implementação dos métodos de extração, são apresentados nas próximas seções.

\subsubsection{Classe para o método de extração MIC (ExtractCIM2D)}

A classe ExtractCIM2D implementa o método da integral de contorno para problemas elásticos bidimensionais. Os principais métodos implementados nessa classe são:

- extract: implementa a formulação numérica do MIC. Nesse método, os pontos de integração são obtidos por meio de objetos da classe Int1d;

- glPoint: implementa a transformação de coordenadas dos pontos de integração. Nesse método, utiliza-se um objeto da classe CoorSysCart;

- jacobian: implementa o jacobiano da transformação de coordenadas para integração numérica;

- normal: implementa o cálculo do vetor unitário normal ao domínio de integração. 
A Figura 5.25 ilustra graficamente o uso das classes do programa SET na implementação do método da integral de contorno.

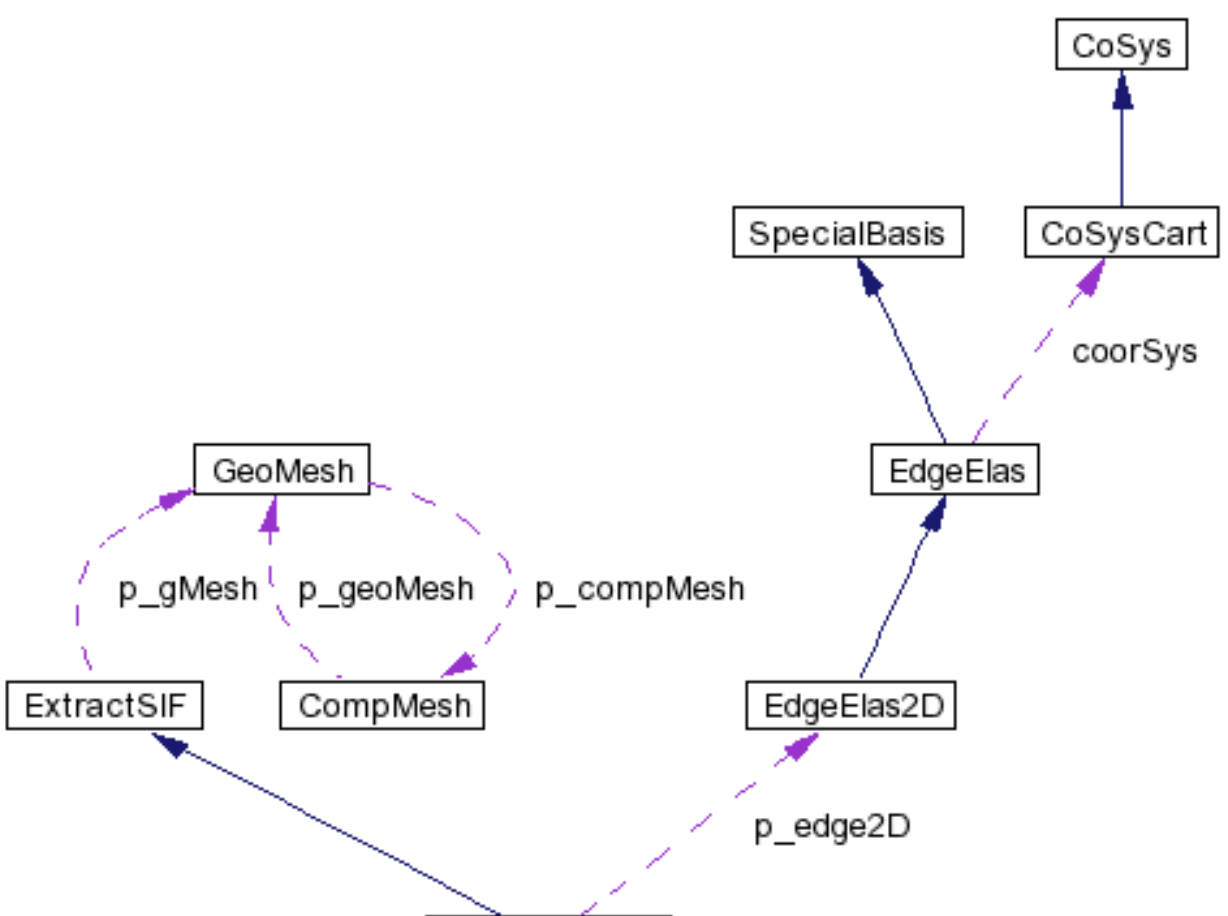

ExtractCIM2D

Figura 5.25 - Representação gráfica da colaboração de outras classes no desenvolvimento da classe ExtractCIM2D.

Para criar um objeto da classe ExtractCIM2D é necessário um objeto da classe GeoMesh e um objeto da classe EdgeElas2D. O objeto da classe ExtractCIM2D fornece a taxa de energia disponibilizada para a propagação da fissura e os fatores de intensidade de tensão para problemas de modos puros e mistos.

\subsubsection{Classe para o método de extração MFC (ExtractCFM2D)}

A classe ExtractCFM2D implementa o método da função cutoff para problemas elásticos bidimensionais. Os principais métodos implementados nessa classe são:

- extract: implementa a formulação numérica do MFC. Nesse método, os pontos de integração são obtidos por meio de objetos da classe Int1d;

- ExtractFunct: implementa a função extratora do MFC. Esse método fornece os deslocamentos da função extratora, suas derivadas de primeira ordem (em 
coordenadas globais) e o integrando da integral de área do MFC. Nesse método, são utilizados objetos da classe CoorSysCart e da classe EdgeElas2D;

- glPoint: implementa a transformação de coordenadas dos pontos de integração. Nesse método, utiliza-se um objeto da classe CoorSysCart;

- jacobianArea: implementa o jacobiano da transformação de coordenadas para a integral de área ;

- jacobianEdge: implementa o jacobiano da transformação de coordenadas para a integral das arestas da fissura;

- normal: implementa o cálculo do vetor unitário normal ao domínio de integração.

A Figura 5.26 ilustra graficamente o uso das classes do programa SET na implementação do método da função cutoff.

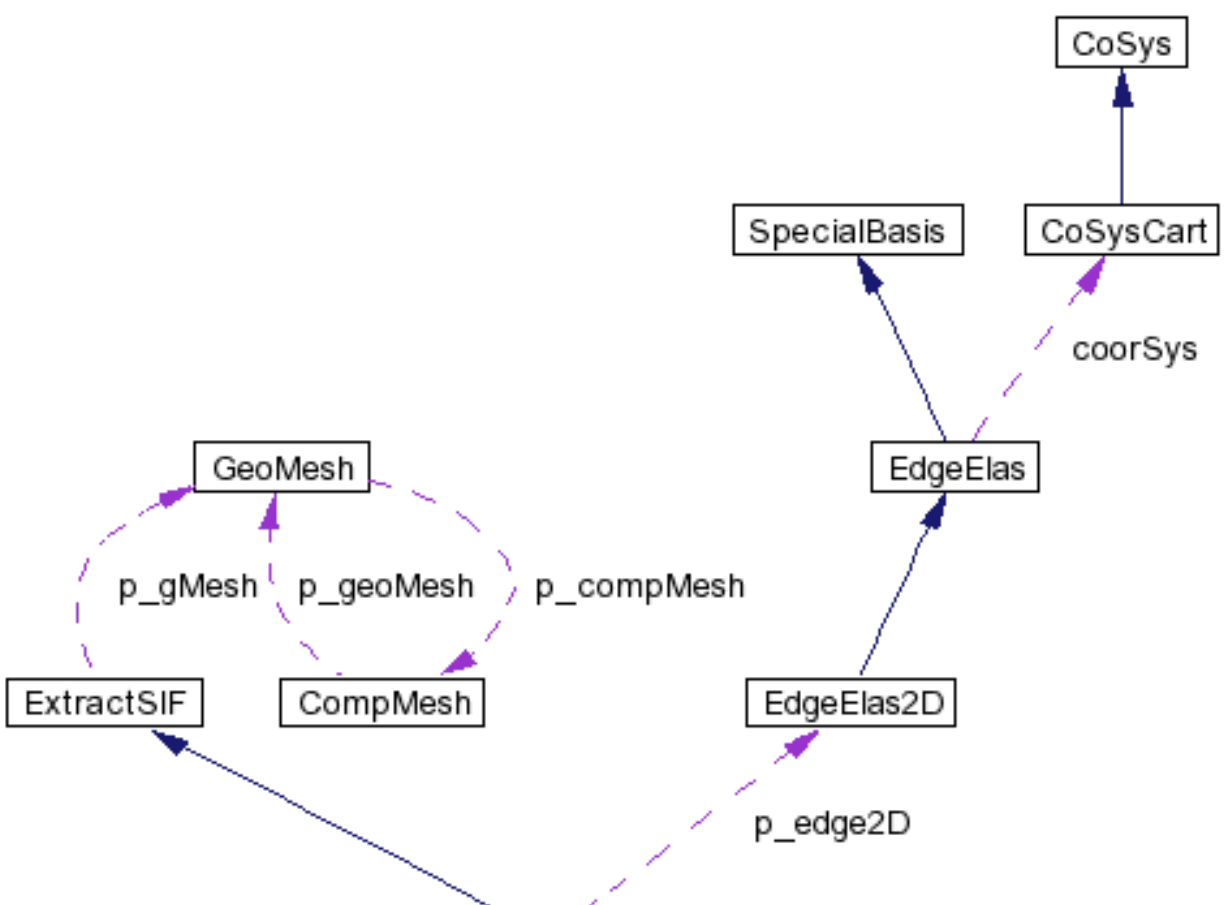

ExtractCFM2D

Figura 5.26 - Representação gráfica da colaboração de outras classes no desenvolvimento da classe ExtractCFM2D.

Para criar um objeto da classe ExtractCFM2D é necessário um objeto da classe GeoMesh e um objeto da classe EdgeElas2D. O objeto da classe ExtractCFM2D 
fornece a taxa de energia disponibilizada para a propagação da fissura e os fatores de intensidade de tensão para problemas de modos puros e mistos.

\subsubsection{Classe para o método da Integral-J (ExtractJ2D)}

A classe ExtractJ2D implementa o método da integral-J para problemas elásticos bidimensionais. Os principais métodos implementados nessa classe são:

- extract: implementa a formulação numérica do método da integral-J. Nesse método os pontos de integração são obtidos por meio de objetos da classe Int1d;

- glPoint: implementa a transformação de coordenadas dos pontos de integração. Nesse método, utiliza-se um objeto da classe CoorSysCart;

- jacobian: implementa o jacobiano da transformação de coordenadas para a integração numérica ;

- normal: implementa o cálculo do vetor unitário normal ao domínio de integração;

A Figura 5.27 ilustra graficamente o uso das classes do programa SET na implementação do método da Integral-J. 


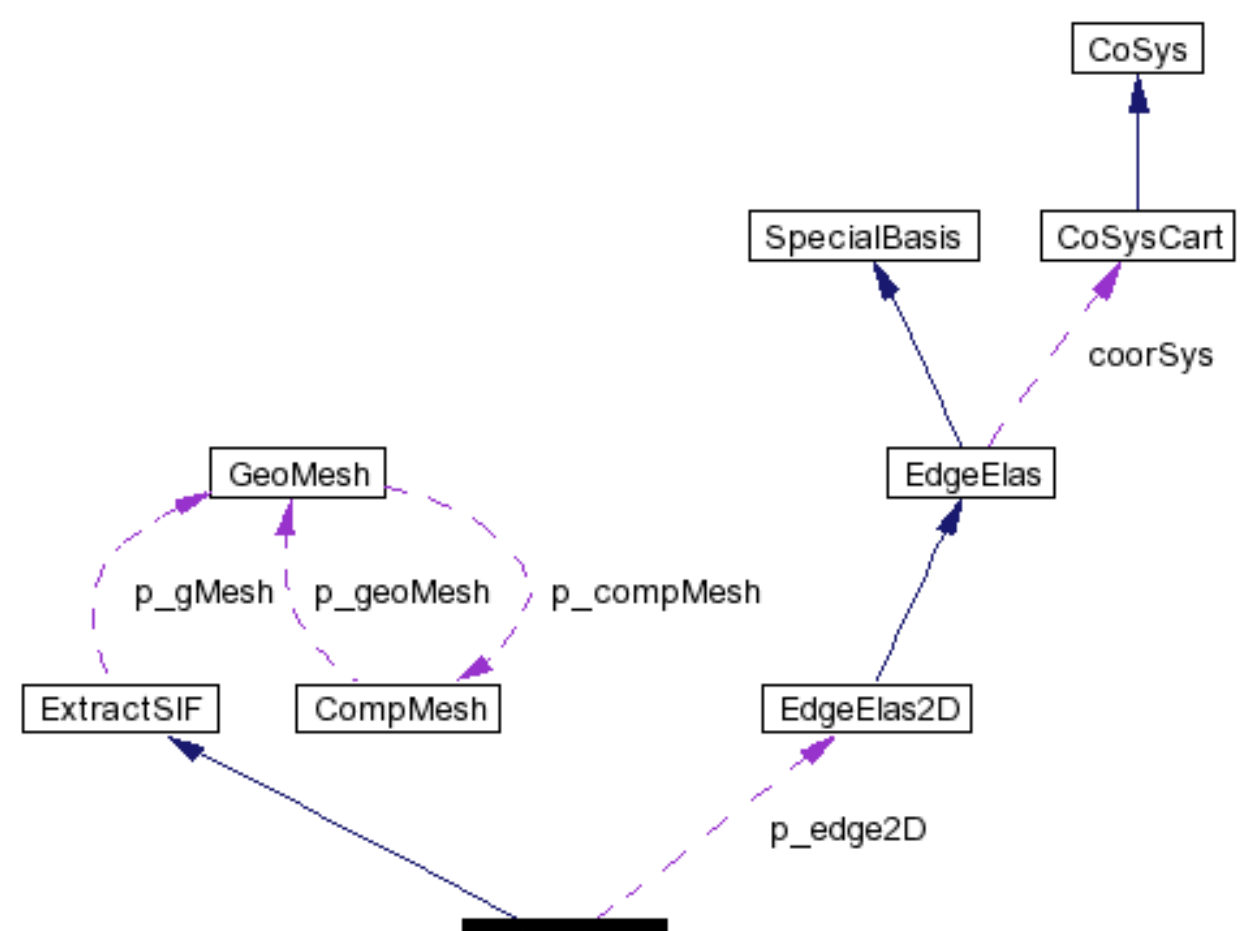

ExtractJ2D

Figura 5.27 - Representação gráfica da colaboração de outras classes no desenvolvimento da classe ExtractJ2D.

Para criar um objeto da classe ExtractJ2D é necessário um objeto da classe GeoMesh e um objeto da classe EdgeElas2D. O objeto da classe ExtractJ2D fornece a taxa de energia disponibilizada para a propagação da fissura e os fatores de intensidade de tensão apenas para modos puros. 


\section{Resultados numéricos}

Neste capítulo apresentam-se os resultados obtidos com a implementação numérica descrita no Capítulo 4. Para tal, foi desenvolvida a solução de problemas clássicos da mecânica da fratura.

A solução numérica utilizada na extração dos fatores de intensidade de tensão foi obtida por meio do programa Scientiffic and Engineering Toolkit (SET) (Capítulo 5).

A análise desenvolvida nesta seção compara o comportamento da convergência dos fatores de intensidade de tensão com a convergência da energia de deformação regida pelo enriquecimento polinomial da solução do MEFG e por malhas apropriadas. Essa análise foi desenvolvida com o intuito de comprovar numericamente a hipótese de superconvergência dos métodos MIC e MFC (Seção 1.4.2).

Os fatores de intensidade de tensão também são comparados com valores numéricos e analíticos encontrados na literatura. Essas análises são desenvolvidas para verificar a robustez e a versatilidade das implementações dos métodos de extração.

Em todos os problemas analisados foram utilizados 12 pontos de Gauss para os métodos MIC e Integral-J e 12 x 12 (144) pontos de Gauss para o método MFC, em todos os exemplos.

\subsection{Painel fraturado com condições de contorno especiais}

Considera-se um problema de painel fraturado como apresenta a Figura 6.1. Idealiza-se o problema da seguinte forma:

- Estado Plano de Deformação (EPD); 
- Coeficiente de Poisson $v=0,3$;

- Módulo de Young $\mathrm{E}=1,0$;

- Dimensões do domínio $\mathrm{a}=1,0$;

- Espessura unitária;

- Condições de contorno de Dirichlet: $\mathrm{u}_{\mathrm{xA}}=0,0, \mathrm{u}_{\mathrm{yA}}=0,0$ e $\mathrm{u}_{\mathrm{yE}}=0,0$;

- Condições de contorno de Neumman: aplicam-se as componentes do tensor de tensão para os modos I (2.29 a 2.31) e II (2.36 a 2.38). As componentes de tensão particularizadas para o problema de painel fraturado são apresentadas a seguir.

No contexto da solução analítica para regiões reentrantes no domínio, apresentada na Seção 2, a fissura do problema de painel fraturado pode ser interpretada como uma região reentrante no domínio de ângulo do material $\alpha=360^{\circ}$. Sendo assim, utilizando o equacionamento da Seção 2, as condições de contorno de tensão podem ser escritas de seguinte forma:

Para modo I, tem-se:

$$
\begin{gathered}
\sigma_{\mathrm{x} 1}^{(1)}=\frac{\mathrm{K}_{\mathrm{I}}}{\sqrt{2 \pi \mathrm{r}}} \cos \left(\frac{\theta}{2}\right)\left[1-\operatorname{sen}\left(\frac{\theta}{2}\right) \operatorname{sen}\left(\frac{3 \theta}{2}\right)\right], \\
\sigma_{\mathrm{y} 1}^{(1)}=\frac{\mathrm{K}_{\mathrm{I}}}{\sqrt{2 \pi \mathrm{r}}} \cos \left(\frac{\theta}{2}\right)\left[1+\operatorname{sen}\left(\frac{\theta}{2}\right) \operatorname{sen}\left(\frac{3 \theta}{2}\right)\right], \\
\tau_{\mathrm{xy} 1}^{(1)}=\frac{\mathrm{K}_{\mathrm{I}}}{\sqrt{2 \pi \mathrm{r}}} \operatorname{sen}\left(\frac{\theta}{2}\right) \cos \left(\frac{\theta}{2}\right) \cos \left(\frac{3 \theta}{2}\right),
\end{gathered}
$$

onde $-\pi \leq \theta \leq \pi$. As componentes para o modo II são:

$$
\begin{gathered}
\sigma_{\mathrm{x} 1}^{(2)}=\frac{\mathrm{K}_{\mathrm{II}}}{\sqrt{2 \pi \mathrm{r}}} \operatorname{sen}\left(\frac{\theta}{2}\right)\left[2+\cos \left(\frac{\theta}{2}\right) \cos \left(\frac{3 \theta}{2}\right)\right], \\
\sigma_{\mathrm{y} 1}^{(2)}=\frac{\mathrm{K}_{\mathrm{II}}}{\sqrt{2 \pi \mathrm{r}}} \operatorname{sen}\left(\frac{\theta}{2}\right) \cos \left(\frac{\theta}{2}\right) \cos \left(\frac{3 \theta}{2}\right),
\end{gathered}
$$




$$
\tau_{\mathrm{xyl}}^{(2)}=\frac{\mathrm{K}_{\mathrm{II}}}{\sqrt{2 \pi \mathrm{r}}} \cos \left(\frac{\theta}{2}\right)\left[1-\operatorname{sen}\left(\frac{\theta}{2}\right) \operatorname{sen}\left(\frac{3 \theta}{2}\right)\right] .
$$

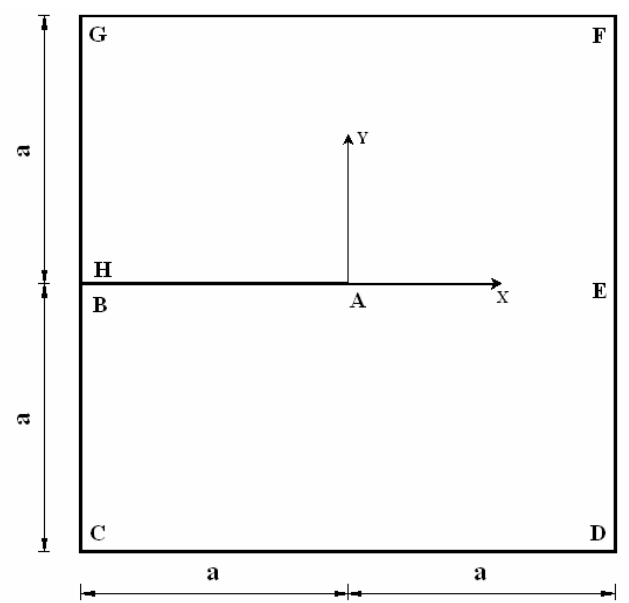

Figura 6.1 - Domínio e eixos coordenados para o problema de painel fraturado.

$\mathrm{Na}$ modelagem computacional do problema foram utilizados elementos quadrilaterais e uma malha geométrica com 3 camadas. Um esboço da malha empregada na análise é apresentado na Figura 6.2.

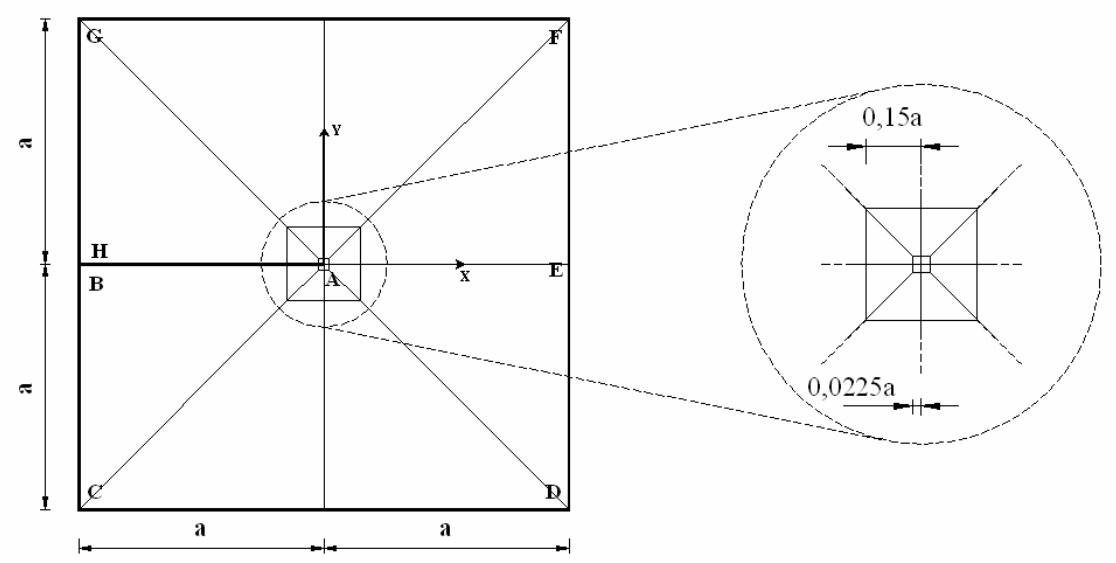

Figura 6.2 - Descrição da malha geométrica utilizada.

Malhas geométricas são comumente empregadas quando se utiliza a versão $\mathrm{p}$ do método de elementos finitos. Para a versão $\mathrm{p}$, a malha geométrica deve ser empregada com uma taxa de progressão $\mathrm{q}=0,15$ em direção ao ponto de singularidade do domínio (SZABÓ (1986); SZABÓ; BABUŠKA (1991)). Oden, Duarte e Zienkiewicz (1998) apresentaram resultados que confirmam a convergência exponencial do MEFG, utilizando malhas geométricas, em problemas com singularidade. Apesar de apresentar 
resultados satisfatórios, não há estudos que comprovam que $\mathrm{q}=0,15$ é a taxa de malha geométrica ideal para o MEFG.

A análise do problema de painel fraturado é apresentada utilizando-se condições de contorno em tensão para modo I (modo puro) e para modos I e II (modo misto). O uso das condições de contorno descritas nessa seção é motivado por não existir solução analítica para problemas da mecânica da fratura em domínio finito. Aplicando-se tais condições, a solução no domínio analisado será correspondente ao modo I ou modos I e II, dependendo das condições de contorno aplicadas. Os resultados obtidos nessas análises são apresentados a seguir. $\mathrm{Na}$ configuração dos domínios de integração utilizou-se $\rho=0,18$ para o MIC e $\rho_{1}=0,18$ e $\rho_{2}=0,60$ para o MFC, para os problemas de Modo I (6.1.1) e Modos I e II (6.1.2).

\subsubsection{Modo I}

Inicialmente aplicam-se apenas as componentes de tensão para modo I. O fator de intensidade de tensão generalizado $\mathrm{A}_{1}{ }^{(1)}$ é descrito em função de $\mathrm{K}_{\mathrm{I}}$ na forma da equação (2.42). Para esse exemplo, considera-se $K_{\mathrm{I}}=\sqrt{2 \pi} \mathrm{A}$ e define-se o fator de intensidade de tensão normalizado $\widetilde{\mathrm{A}}_{1}{ }^{(1)}$ na forma:

$$
\widetilde{\mathrm{A}}_{1}^{(1)}=\frac{\left(\mathrm{A}_{1}^{(1)}\right)_{\mathrm{EFG}}}{\mathrm{A}},
$$

onde A é um valor arbitrário. Sendo assim, os valores calculados com os métodos de extração devem convergir para 1,0. Essa normalização facilita a monitoração da convergência dos fatores de intensidade de tensão.

A energia de deformação exata para o presente problema é dada por (DUARTE, 1991):

$$
\mathrm{U}\left(\mathrm{u}_{\mathrm{EX}}\right)=2,979042 \frac{\mathrm{A}^{2} \mathrm{at}_{\mathrm{z}}}{\mathrm{E}}
$$

onde $\mathrm{t}_{\mathrm{z}}$ é a espessura da chapa.

A Figura 6.3 ilustra as condições de contorno aplicadas e a malha utilizada na presente análise. 


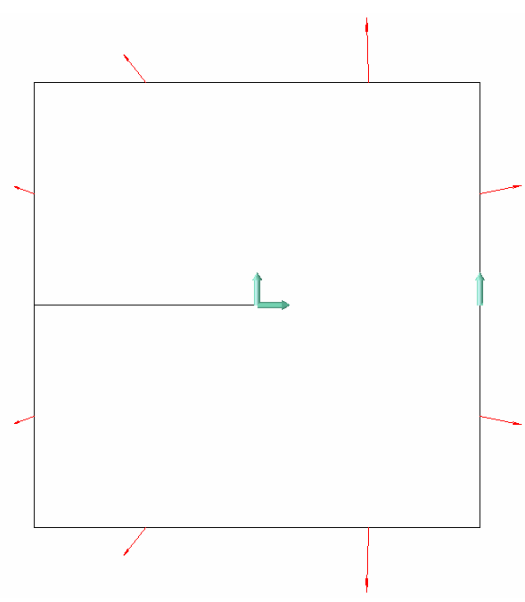

(a)

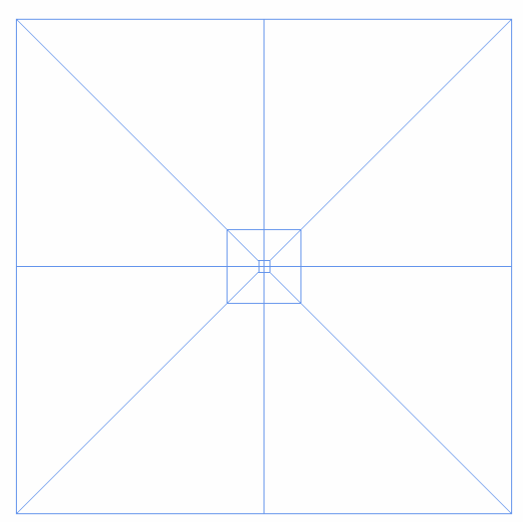

(b)

Figura 6.3 - (a) Condições de contorno aplicadas (tensões modo I) e (b) malha utilizada na discretização.

Szabó e Babuška (1988) apresentaram uma análise do erro relativo dos fatores de intensidade comparando sua convergência com o erro relativo na energia de deformação utilizando a versão $\mathrm{p}$ do MEF e malhas geométricas. Com o intuito de validar o uso do MEFG em uma análise de convergência semelhante, avalia-se a convergência do erro na norma energia $\left(\operatorname{er}\left(\|U\|_{E}\right)\right)$ e na energia de deformação (er(U)), obtidos com o MEFG, comparando com os resultados obtidos por Duarte (1991) utilizando a versão $p$ do $M E F$. er $\left(\|U\|_{E}\right)$ e er(U) são dados por:

$$
\begin{gathered}
\operatorname{er}\left(\|\mathrm{U}\|_{\mathrm{E}}\right)=\frac{\left\|\mathrm{U}-\mathrm{U}_{\mathrm{EFG}}\right\|_{\mathrm{E}}}{\|\mathrm{U}\|_{\mathrm{E}}}, \\
\operatorname{er}(\mathrm{U})=\frac{\mathrm{U}-\mathrm{U}_{\mathrm{EFG}}}{\mathrm{U}},
\end{gathered}
$$

onde \|\|$_{E}$ é a norma energia, $U$ é o valor de referência para energia e $U_{E F G}$ é a energia obtida com a solução do MEFG.

As Tabelas 6.1 e 6.2 apresentam os valores para os erros relativos obtidos nas análises utilizando a versão p do MEF e o MEFG, respectivamente. Em ambos os casos foi utilizada uma malha geométrica com 3 camadas e elementos quadrilaterais, como apresentado na Figura 6.2. 
Tabela 6.1 - Valores de er(U) e er $\left(\|U\|_{E}\right)$ para a solução da versão p do MEF (problema de painel fraturado utilizando condições de contorno para modo I) (DUARTE, 1991).

\begin{tabular}{ccccc}
\hline $\mathrm{p}$ & $\mathrm{N}$ & $\mathrm{U}_{\text {MEF-p }}$ & $\begin{array}{c}\mathrm{er}(\mathrm{U}) \% \\
\mathrm{MEF}-\mathrm{p}\end{array}$ & $\begin{array}{c}\mathrm{er}\left(\|\mathrm{U}\|_{\mathrm{E}}\right) \% \\
\mathrm{MEF}-\mathrm{p}\end{array}$ \\
\hline 1 & 64 & 2,636678 & 11,49 & 33,90 \\
2 & 164 & 2,928155 & 1,70 & 13,06 \\
3 & 264 & 2,961924 & 0,57 & 7,58 \\
4 & 404 & 2,970030 & 0,30 & 5,50 \\
5 & 584 & 2,973162 & 0,19 & 4,44 \\
6 & 804 & 2,974810 & 0,14 & 3,76 \\
7 & 1064 & 2,975827 & 0,10 & 3,28 \\
8 & 1364 & 2,976514 & 0,08 & 2,91 \\
\hline$\infty$ & & 2,979042 & \\
\hline
\end{tabular}

Tabela 6.2 - Valores de er(U) e er $\left(\|\mathrm{U}\|_{\mathrm{E}}\right)$ para a solução do MEFG (problema de painel fraturado utilizando condições de contorno para modo I).

\begin{tabular}{ccccc}
\hline $\mathrm{p}$ & $\mathrm{N}$ & $\mathrm{U}_{\mathrm{MEFG}}$ & $\begin{array}{c}\mathrm{er}(\mathrm{U}) \% \\
\mathrm{MEFG}\end{array}$ & $\begin{array}{c}\operatorname{er}\left(\|\mathrm{U}\|_{\mathrm{E}}\right) \% \\
\mathrm{MEFG}\end{array}$ \\
\hline 1 & 56 & 2,726045 & 8,49 & 29,14 \\
2 & 168 & 2,944589 & 1,15 & 10,75 \\
3 & 336 & 2,972140 & 0,23 & 4,81 \\
4 & 560 & 2,976227 & 0,09 & 3,07 \\
5 & 840 & 2,976647 & 0,08 & 2,83 \\
6 & 1176 & 2,976935 & 0,07 & 2,66 \\
7 & 1568 & 2,976916 & 0,07 & 2,67 \\
8 & 2016 & 2,977125 & 0,06 & 2,53 \\
\hline$\infty$ & & 2,979042 & \\
\hline
\end{tabular}

A Figura 6.4 apresenta o gráfico do erro relativo na norma energia e na energia de deformação utilizando versão $\mathrm{p}$ do MEF e MEFG variando com número de graus de liberdade. 


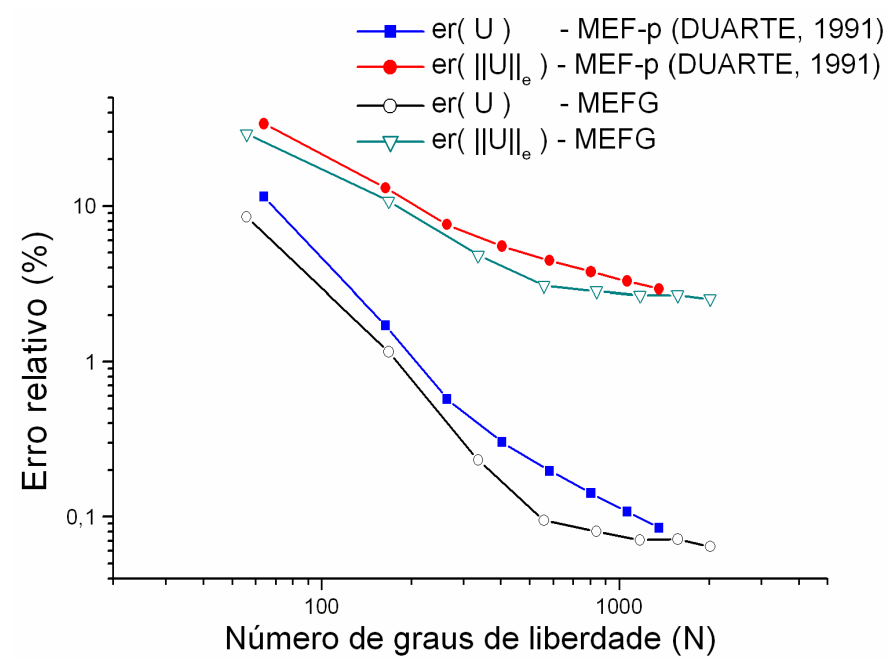

Figura 6.4 - Erro relativo na norma energia e na energia de deformação para a versão $p$ do MEF e para o MEFG (escala $\log x \log$ ).

A Figura 6.4 indica que, para uma mesma malha, os erros relativos obtidos com o MEFG e com a versão-p do MEF são praticamente equivalentes. A curva S invertido, típica da convergência da norma energia utilizando a versão p do MEF (SZABÓ e BABUŠKA, 1991), é claramente visível também no MEFG.

A Figura 6.5 apresenta a magnitude do vetor deslocamento e a tensão de von Mises obtidas com o MEFG para o problema de painel fraturado com modo I.

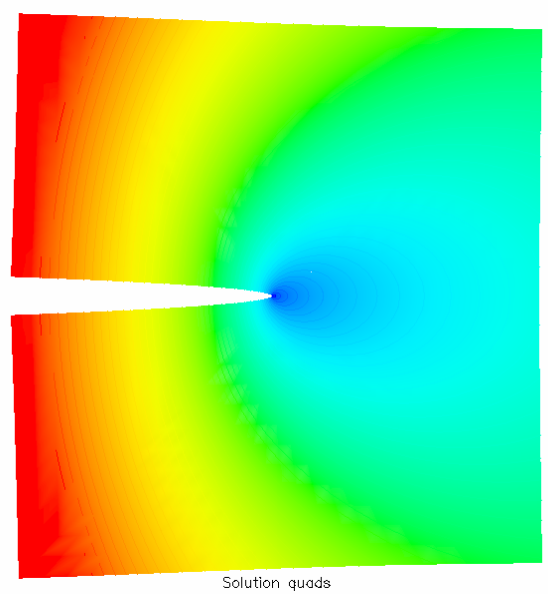

(a)

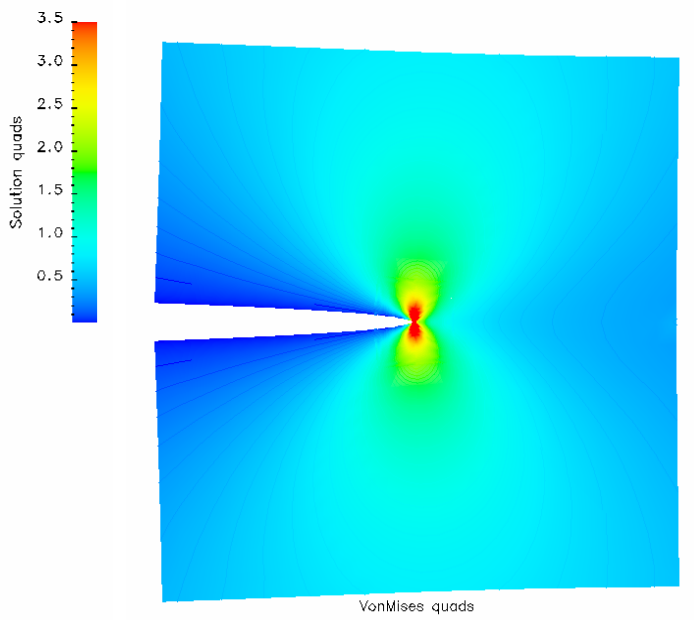

(b)

Figura 6.5 - (a) Magnitude do vetor deslocamento e (b) tensão de von Mises para o problema de painel fraturado utilizando as condições de contorno para modo I.

A taxa de energia disponibilizada para a propagação da fissura, para problemas planos e elásticos lineares, pode ser escrita da seguinte forma (OWEN; FAWKES, 1983): 


$$
\mathscr{G}=\mathrm{J}=\frac{(1+\kappa)}{8 \mathrm{G}}\left(\mathrm{K}_{\mathrm{I}}^{2}+\mathrm{K}_{\mathrm{II}}^{2}\right)
$$

Tratando-se de um problema de modo puro, utilizando a equação (6.11), é possível avaliar também o fator de intensidade de tensão obtido por meio do método da integral-J, sem procedimentos adicionais (MORAN; SHIH, 1987a, b; NAHTA; MORAN, 1993; ORGAN, 1996; RAJU; SHIVAKUMAR, 1990).

$\mathrm{Na}$ Tabela 6.3 encontram-se os valores dos fatores de intensidade de tensão normalizados obtidos com os métodos MIC, MFC e Integral-J. A Figura 6.6 apresenta uma comparação da convergência entre os erros relativos na norma energia, na energia de deformação e nos fatores de intensidade de tensão.

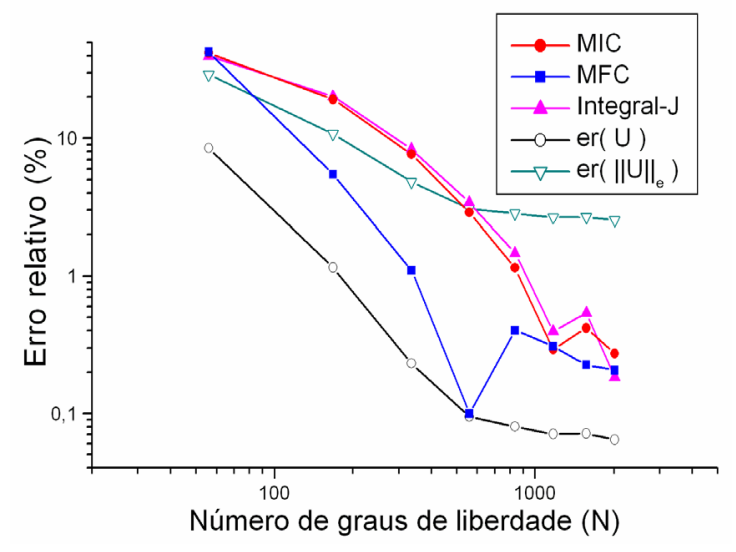

(a)

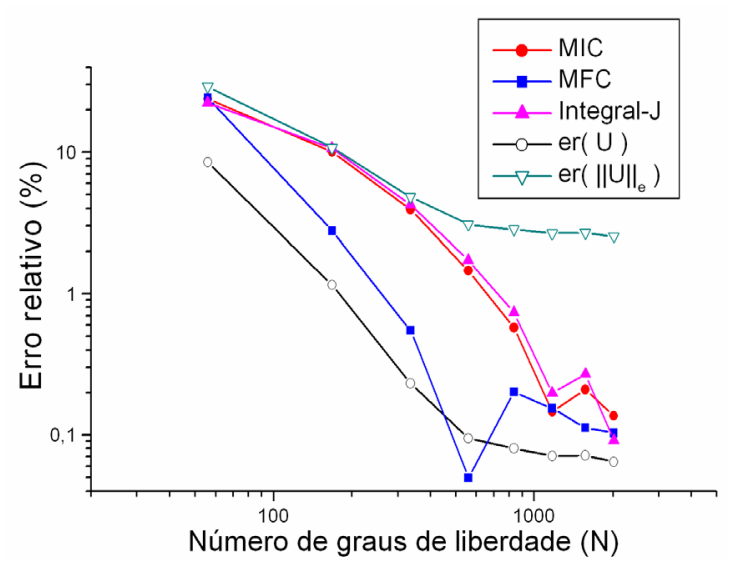

(b)

Figura 6.6 - (a) Taxa de energia disponibilizada - Erro relativo (\%) X Número de graus de liberdade (escala $\log x \log$ ) e (b) Fator de intensidade de tensão $\mathrm{K}_{\mathrm{I}}$ - Erro relativo (\%) X Número de graus de liberdade (escala $\log x \log$ ).

A Tabela 6.3 mostra que os resultados com o MFC são mais precisos do que os resultados obtidos com o MIC e com a Integral-J. Para o problema analisado, é possível obter um erro relativo menor ou igual a $1,0 \%$ enriquecendo a solução com $p=3$ e utilizando o MFC. Para obter o mesmo nível de precisão utilizando os métodos MIC e Integral-J, faz-se necessário enriquecer a solução com $\mathrm{p}=5$. 
Tabela 6.3 - Fator de intensidade de tensão normalizado e erro relativo (\%) obtidos com MIC, MFC e integral-J.

\begin{tabular}{ccccccc}
\hline $\mathrm{N}$ & $\widetilde{\mathrm{A}}_{1}^{(1)}$ & $\operatorname{er}\left(\widetilde{\mathrm{A}}_{1}^{(1)}\right) \%$ & $\widetilde{\mathrm{A}}_{1}^{(1)}$ & $\operatorname{er}\left(\widetilde{\mathrm{A}}_{1}^{(1)}\right) \%$ & $\widetilde{\mathrm{A}}_{1}^{(1)}$ & $\operatorname{er}\left(\widetilde{\mathrm{A}}_{1}{ }^{(1)}\right) \%$ \\
& $\mathrm{~J}$ & $\mathrm{~J}$ & $\mathrm{MIC}$ & $\mathrm{MIC}$ & $\mathrm{MFC}$ & $\mathrm{MFC}$ \\
\hline 56 & 0,775866 & 22,4134 & 0,761677 & 23,8323 & 0,757029 & 24,2971 \\
168 & 0,893418 & 10,6582 & 0,899090 & 10,0910 & 0,972210 & 2,7790 \\
336 & 0,957515 & 4,2485 & 0,960914 & 3,9086 & 1,005503 & 0,5503 \\
560 & 0,982788 & 1,7212 & 0,985427 & 1,4573 & 1,000497 & 0,0497 \\
840 & 0,992648 & 0,7352 & 0,994246 & 0,5754 & 0,997983 & 0,2017 \\
1176 & 0,998019 & 0,1981 & 0,998537 & 0,1463 & 0,998456 & 0,1544 \\
1568 & 0,997301 & 0,2699 & 0,997906 & 0,2094 & 0,998872 & 0,1128 \\
2016 & 0,999083 & 0,0917 & 0,998634 & 0,1366 & 0,998968 & 0,1032 \\
\hline$\infty$ & 1,000000 & \multicolumn{7}{c}{1,000000} & 1,000000 & \\
\hline
\end{tabular}

Na Figura 6.6 observa-se que os métodos da Integral-J e MIC apresentam um comportamento semelhante. Isso ocorre porque ambos os métodos possuem sua formulação baseada em integrais de contorno, avaliadas na vizinhança da região singular. Também é possível observar que os métodos MIC e MFC apresentam características de superconvergência, pois suas taxas de convergências são iguais ou maiores do que a taxa da energia de deformação, portanto, superiores a convergência da solução na norma energia.

Para o MFC, observa-se que a curva do erro relativo para o fator de intensidade de tensão (Figura 6.6 (b)) apresenta-se no regime pré-assintótico (de $\mathrm{p}=1$ a $\mathrm{p}=4$ ) com uma taxa de convergência maior do que a taxa de convergência da energia de deformação. No regime assintótico (de $p=5$ a $p=8$ ) o MFC apresenta uma taxa de convergência semelhante a da energia de deformação. Estas observações mostram que os resultados são condizentes com as teorias propostas por Babuška e Miller (1984b, c) e apresentadas na Seção 1.4.2.

A Figura 4.3 e a Tabela 6.4 apresentam uma verificação numérica da independência do caminho de integração para os três métodos de extração implementados. Nessa verificação, utilizou-se um enriquecimento polinomial de ordem $p=7$. Para o método MFC adotou-se o raio interno $\rho_{1}=0,05$ e o raio externo $\left(\rho_{2}\right)$ variou conforme o raio de integração $(\rho)$ dos métodos MIC e da Integral-J. 


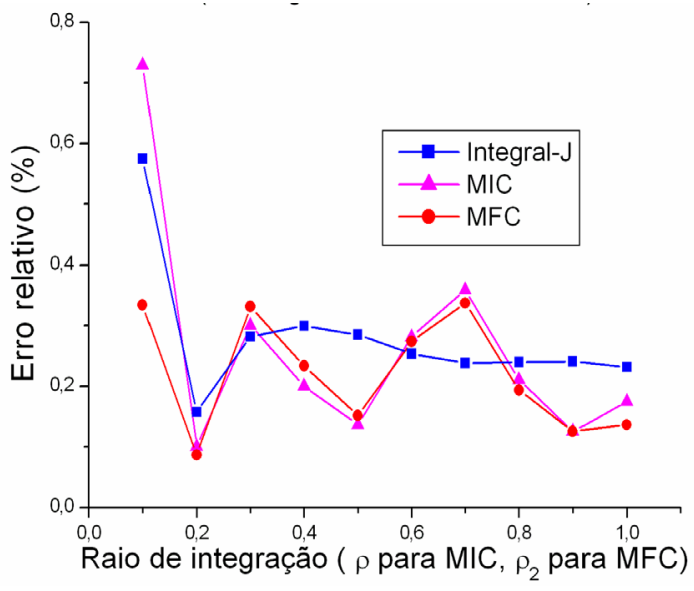

(a)

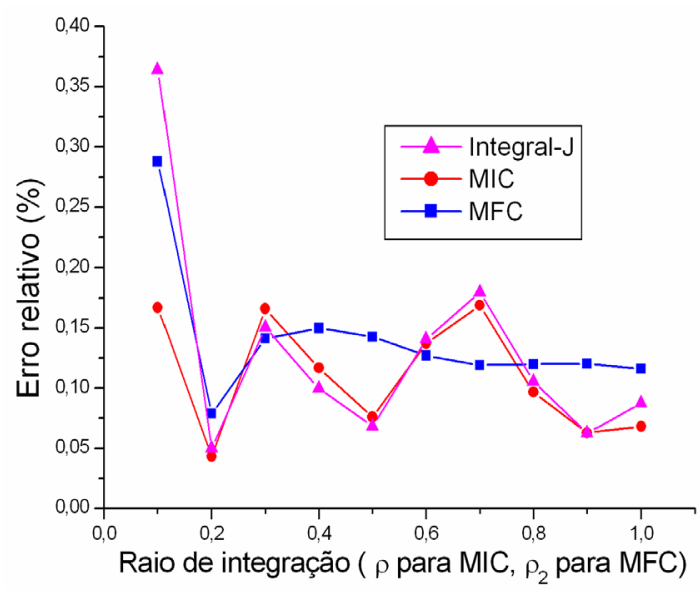

(b)

Figura 6.7 - (a) Taxa de energia disponibilizada - Erro relativo (\%) X raio de integração e (b) Fator de intensidade de tensão $\mathrm{K}_{\mathrm{I}}-$ Erro relativo (\%) $\mathrm{X}$ raio de integração.

Tabela 6.4 - Verificação da independência do caminho.

\begin{tabular}{cccc}
\hline $\mathrm{R}$ & $\begin{array}{c}\operatorname{er}\left(\widetilde{\mathrm{A}}_{1}^{(1)}\right) \% \\
\mathrm{~J}\end{array}$ & $\begin{array}{c}\operatorname{er}\left(\widetilde{\mathrm{A}}_{1}^{(1)}\right) \% \\
\text { MIC }\end{array}$ & $\begin{array}{c}\operatorname{er}\left(\widetilde{\mathrm{A}}_{1}^{(1)}\right) \% \\
\text { MFC }\end{array}$ \\
\hline 0,1 & 0,3639 & 0,1669 & 0,2878 \\
0,2 & 0,0501 & 0,0433 & 0,0788 \\
0,3 & 0,1503 & 0,1660 & 0,1412 \\
0,4 & 0,0999 & 0,1169 & 0,1499 \\
0,5 & 0,0682 & 0,0759 & 0,1427 \\
0,6 & 0,1407 & 0,1372 & 0,1271 \\
0,7 & 0,1798 & 0,1688 & 0,1191 \\
0,8 & 0,1054 & 0,0968 & 0,1199 \\
0,9 & 0,0627 & 0,0628 & 0,1205 \\
1,0 & 0,0875 & 0,0682 & 0,1159 \\
\hline Mín & 0,0501 & 0,0433 & 0,0788 \\
\hline Média & 0,1308 & 0,1103 & 0,1403 \\
\hline Máx & 0,3639 & 0,1688 & 0,2878 \\
\hline
\end{tabular}

No presente problema, onde as condições de contorno são as componentes de tensão apresentadas nas equações de (6.1) a (6.3), a solução em todo o domínio é regida pela primeira parcela simétrica da expansão assintótica. Sob estas condições, todos os métodos implementados apresentaram rendimento satisfatório com respeito à independência do caminho de integração.

\subsubsection{Modo I e Modo II}

Nessa análise são consideradas as componentes de tensão para modo I e modo II. O fatores de intensidade de tensão generalizados $A_{1}^{(1)}$ e $A_{1}^{(2)}$ são descritos em 
função de $K_{I}$ e $K_{I I}$ na forma da equação (2.42). Para esse exemplo, considera-se $\mathrm{K}_{\mathrm{I}}=\mathrm{K}_{\mathrm{II}}=\sqrt{2 \pi} \mathrm{A}$ e os fatores de intensidade de tensão normalizado $\widetilde{\mathrm{A}}_{1}{ }^{(1)}$ e $\widetilde{\mathrm{A}}_{1}{ }^{(2)}$ são definidos na forma:

$$
\widetilde{\mathrm{A}}_{1}^{(1)}=\frac{\left(\mathrm{A}_{1}^{(1)}\right)_{\mathrm{EFG}}}{\mathrm{A}} ; \quad \widetilde{\mathrm{A}}_{1}^{(2)}=\frac{\left(\mathrm{A}_{1}^{(2)}\right)_{\mathrm{EFG}}}{\mathrm{A}},
$$

onde A é um valor arbitrário. Sendo assim, os valores calculados com os métodos de extração devem convergir para 1,0. Essa normalização facilita a monitoração da convergência dos fatores de intensidade de tensão.

A energia de deformação exata para o presente problema é dada por:

$$
\mathrm{U}\left(\mathrm{u}_{\mathrm{EX}}\right)=10,5412281 \frac{\mathrm{A}^{2} \mathrm{at}_{\mathrm{z}}}{\mathrm{E}}
$$

onde $\mathrm{t}_{\mathrm{z}}$ é a espessura do painel (SZABÓ; BABUŠKA, 1988).

A Figura 6.8 ilustra as condições de contorno aplicadas e a malha utilizada na análise do problema de painel fraturado considerando os modos I e II.

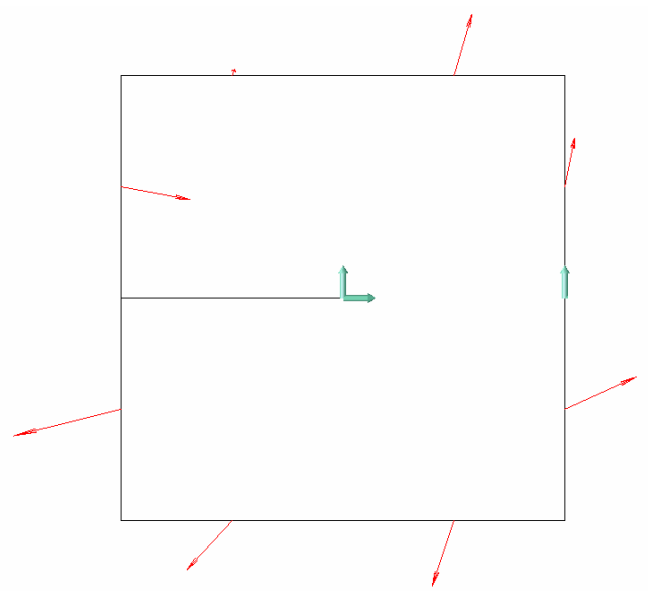

(a)

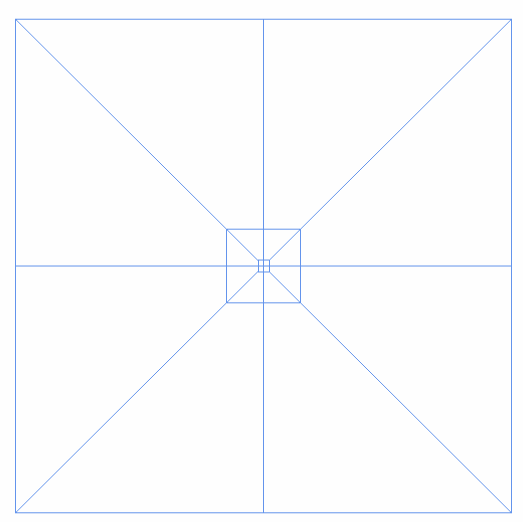

(b)

Figura 6.8 - (a) Condições de contorno aplicadas (tensões modos I e II) e (b) malha utilizada na discretização.

A Figura 6.9 apresenta a magnitude do vetor deslocamento e a tensão de von Mises obtidas com o MEFG para o problema de painel fraturado submetido as tensões dos modos I e II. 


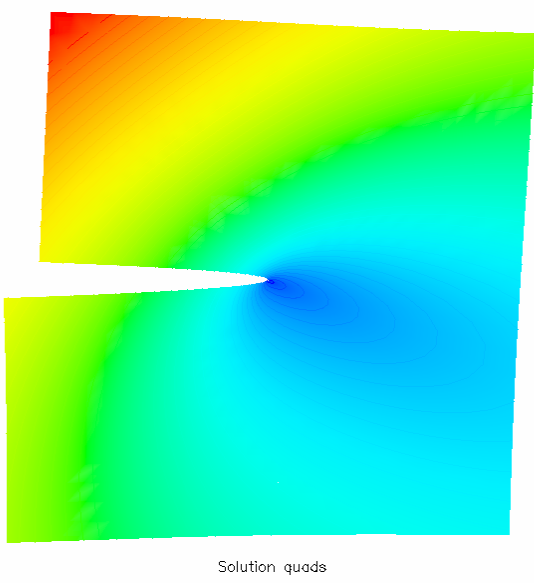

(a)
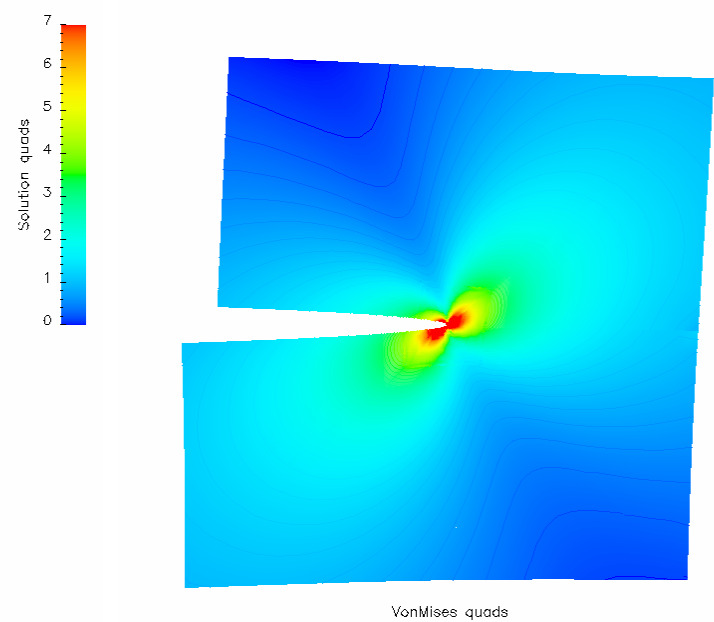

(b)

Figura 6.9 - (a) Magnitude do vetor deslocamento e (b) tensão de von Mises para o problema de painel fraturado utilizando as condições de contorno para modos I e II.

A Tabela 6.5 apresenta os valores da energia de deformação $\left(U_{E F G}\right)$ e os valores do erro relativo na norma energia e na energia de deformação.

Tabela 6.5 - Valores de er(U) e er $\left(\|\mathrm{U}\|_{\mathrm{E}}\right)$ para a solução do MEFG (problema de painel fraturado utilizando condições de contorno para modos I e II).

\begin{tabular}{ccccc}
\hline $\mathrm{p}$ & $\mathrm{N}$ & $\mathrm{U}_{\mathrm{MEFG}}$ & $\begin{array}{c}\operatorname{er}(\mathrm{U}) \% \\
\mathrm{MEFG}\end{array}$ & $\begin{array}{c}\operatorname{er}\left(\|\|_{\mathrm{E}}\right) \% \\
\mathrm{MEFG}\end{array}$ \\
\hline 1 & 56 & 9,76223 & 7,39 & 27,18455 \\
2 & 168 & 10,43097 & 1,046 & 10,22741 \\
3 & 336 & 10,51241 & 0,2734 & 5,228767 \\
4 & 560 & 10,52586 & 0,1458 & 3,818377 \\
5 & 840 & 10,52838 & 0,1219 & 3,491418 \\
6 & 1176 & 10,52892 & 0,1167 & 3,416138 \\
7 & 1568 & 10,52899 & 0,1161 & 3,407345 \\
8 & 2016 & 10,53058 & 0,101 & 3,17805 \\
\hline & \multicolumn{5}{|}{} \\
\hline
\end{tabular}

Nas Tabelas 6.6 e 6.7 encontram-se os valores dos fatores de intensidade de tensão normalizados, obtidos com os métodos MIC e MFC, para os modos I e II, respectivamente. A Figura 6.10 apresenta uma comparação da convergência entre os erros relativos na norma energia, na energia de deformação e nos fatores de intensidade de tensão. 
Tabela 6.6 - Fator de intensidade de tensão normalizado e erro relativo (\%) obtidos com MIC, MFC (Modo I).

\begin{tabular}{ccccc}
\hline $\mathrm{N}$ & $\widetilde{\mathrm{A}}_{1}^{(1)}$ & $\operatorname{er}\left(\tilde{\mathrm{A}}_{1}^{(1)}\right) \%$ & $\widetilde{\mathrm{A}}_{1}^{(1)}$ & $\operatorname{er}\left(\widetilde{\mathrm{A}}_{1}^{(1)}\right) \%$ \\
& $\mathrm{MIC}$ & $\mathrm{MIC}$ & $\mathrm{MFC}$ & $\mathrm{MFC}$ \\
\hline 56 & 0,761677 & 23,8323 & 0,757029 & 24,2971 \\
168 & 0,899090 & 10,0910 & 0,972210 & 2,7790 \\
336 & 0,960914 & 3,9086 & 1,005503 & 0,5503 \\
560 & 0,985427 & 1,4573 & 1,000497 & 0,0497 \\
840 & 0,994246 & 0,5754 & 0,997983 & 0,2017 \\
1176 & 0,998537 & 0,1463 & 0,998456 & 0,1544 \\
1568 & 0,997906 & 0,2094 & 0,998872 & 0,1128 \\
2016 & 0,998634 & 0,1366 & 0,998968 & 0,1032 \\
\hline$\infty$ & 1,000000 & \multicolumn{3}{c}{1,000000} \\
\hline
\end{tabular}

Tabela 6.7 - Fator de intensidade de tensão normalizado e erro relativo (\%) obtidos com MIC, MFC (Modo II).

\begin{tabular}{ccccc}
\hline $\mathrm{N}$ & $\widetilde{\mathrm{A}}_{1}^{(2)}$ & $\operatorname{er}\left(\widetilde{\mathrm{A}}_{1}^{(2)}\right) \%$ & $\widetilde{\mathrm{A}}_{1}^{(2)}$ & $\operatorname{er}\left(\widetilde{\mathrm{A}}_{1}^{(2)}\right) \%$ \\
& $\mathrm{MIC}$ & $\mathrm{MIC}$ & $\mathrm{MFC}$ & $\mathrm{MFC}$ \\
\hline 56 & 0,819702 & 18,0298 & 0,733376 & 26,6624 \\
168 & 0,861264 & 13,8736 & 0,973867 & 2,6133 \\
336 & 0,947477 & 5,2523 & 1,010037 & 1,0037 \\
560 & 0,981256 & 1,8744 & 1,003715 & 0,3715 \\
840 & 0,991196 & 0,8804 & 0,999215 & 0,0785 \\
1176 & 0,995839 & 0,4161 & 0,998491 & 0,1509 \\
1568 & 1,000212 & 0,0212 & 0,998646 & 0,1354 \\
2016 & 0,999145 & 0,0855 & 0,998868 & 0,1132 \\
\hline$\infty$ & 1,000000 & & 1,000000 & \\
\hline
\end{tabular}

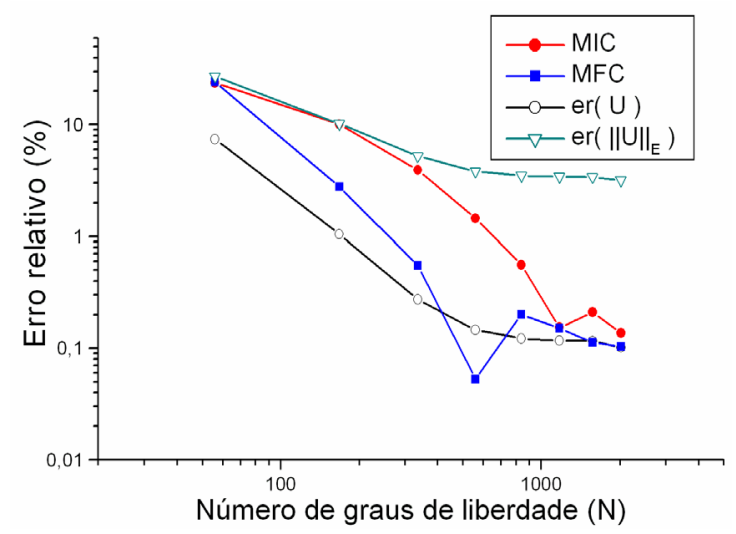

(a)

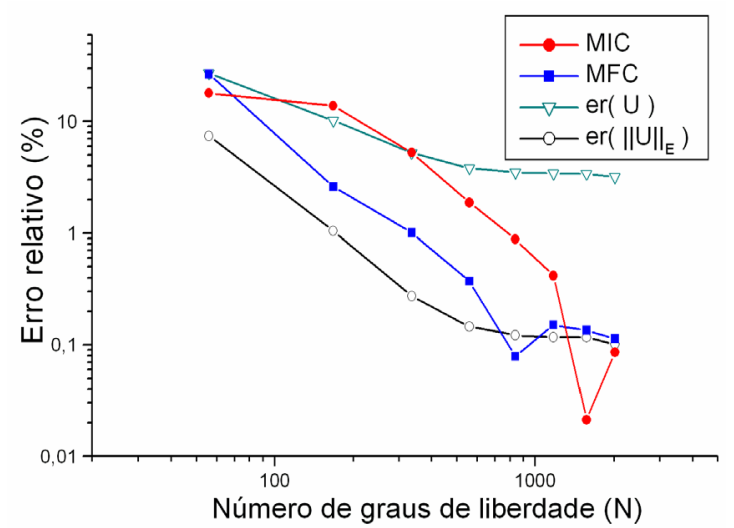

(b)

Figura 6.10 - (a) Fator de intensidade de tensão $\mathrm{K}_{\mathrm{I}}$ - Erro relativo (\%) X Número de graus de liberdade (escala $\log \mathrm{x} \log$ ) e (b) Fator de intensidade de tensão $\mathrm{K}_{\mathrm{II}}-$ Erro relativo (\%) X Número de graus de liberdade (escala log x log). 
Os resultados apresentados nas Tabelas 6.6 e 6.7 mostram que os fatores de intensidade de tensão obtidos com o MFC, tanto para modo I quanto para o modo II, apresentam-se de forma mais precisa do que no MIC. Utilizando-se o MFC, para os fatores de intensidade de tensão de ambos os modos, é possível obter um erro relativo menor ou igual a $1,0 \%$ assumindo-se $\mathrm{p}=3$, enquanto que para o mesmo erro relativo utilizando o MIC é necessário assumir $p=5$. Com relação ao erro relativo, tanto o método MFC quanto o método MIC apresentaram erros relativos menores do que o erro relativo na norma energia. As taxas de convergência para os métodos MIC e MFC são iguais ou maiores do que as taxas de convergência da energia de deformação, portanto, superiores a convergência da solução na norma energia.

A Figura 6.11 apresenta o erro relativo da taxa de energia disponibilizada para a propagação da fissura obtido com os métodos MIC, MFC e Integral-J.

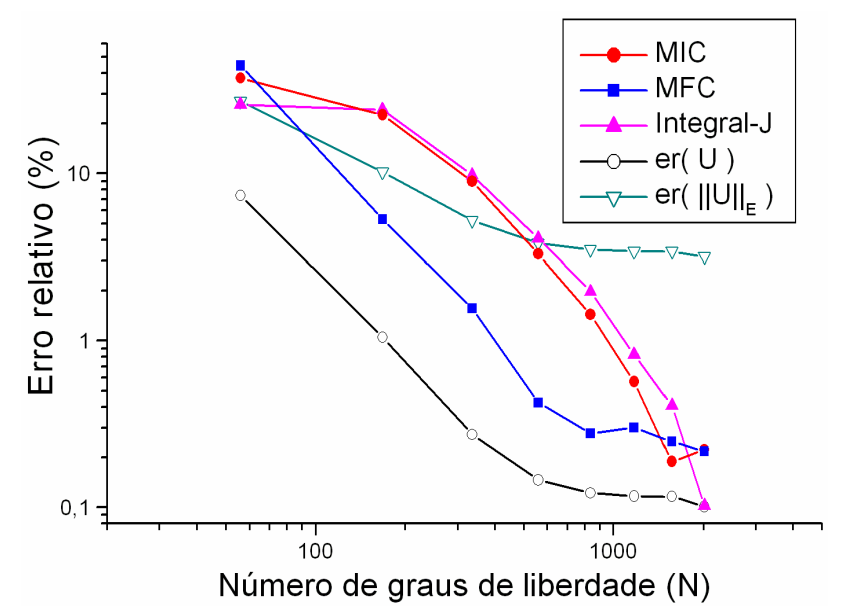

Figura 6.11 - Taxa de energia disponibilizada - Erro relativo (\%) X Número de graus de liberdade (escala $\log x \log$ ).

Assim como no exemplo 6.1.1, os resultados obtidos com os métodos MIC e Integral-J apresentam comportamentos semelhantes (Figura 6.11). A formulação para o MIC e Integral-J é baseada em integrais de contorno que utilizam a solução aproximada do MEFG na vizinhança da fissura. Como esperado, o MFC apresentou melhores resultados tanto para a extração dos fatores de intensidade de tensão quanto para a taxa de energia disponibilizada para a propagação da fissura.

As Figuras 6.12 e 6.13 e as Tabelas 6.8 e 6.9 apresentam uma verificação da independência do caminho de integração na extração dos fatores de intensidade de tensão e no cálculo da taxa de energia disponibilizada para a propagação da fissura. 
Adotou-se a variação dos raios da mesma forma que foi desenvolvida nas verificações para a independência do caminho no problema 6.1.1.

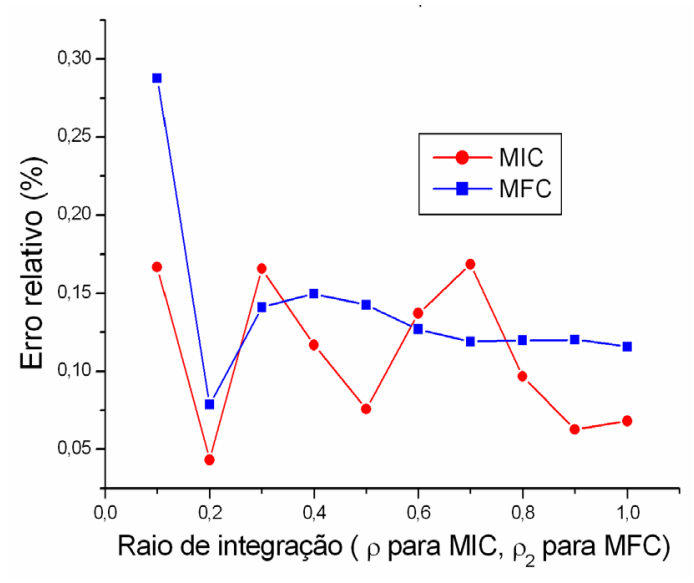

(a)

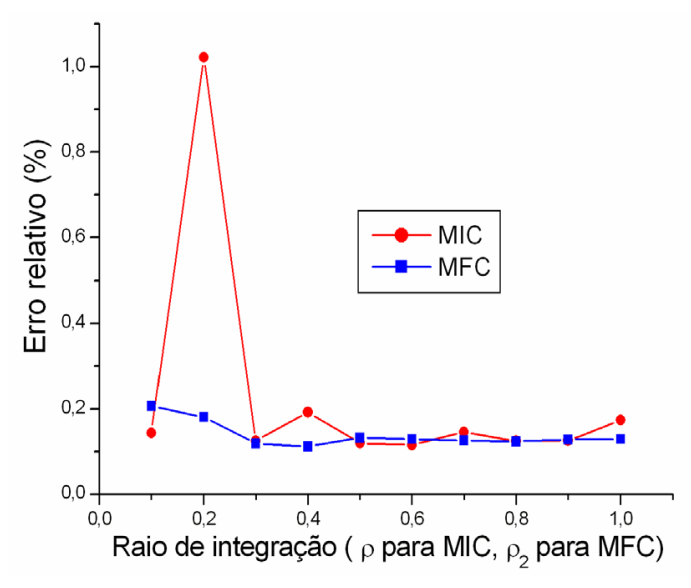

(b)

Figura 6.12 - (a) Fator de intensidade de tensão $K_{I}$ - Erro relativo (\%) X Raio de integração (R) e (b) Fator de intensidade de tensão $K_{I I}-$ Erro relativo (\%) X Raio de integração (R).

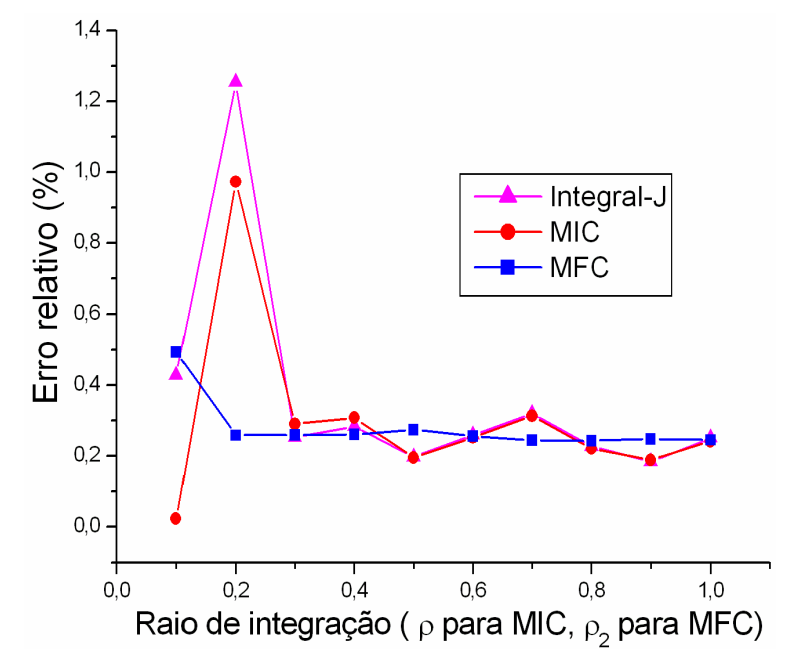

Figura 6.13 - Taxa de energia disponibilizada - Erro relativo (\%) X Número de graus de liberdade (escala log x log).

Nos resultados apresentados na Figura 6.12 e na Figura 6.13 é possível observar que os método da Integral-J e da integral de contorno são mais sensíveis à variação do raio quando se utiliza a solução dos elementos que estão muito próximos a ponta da fissura $[\mathrm{r}=0,1 ; 0,3]$. O método da função cutoff não apresenta variação significativa no mesmo intervalo. 
Tabela 6.8 - Verificação da independência do caminho (Erro relativo para os fatores de intensidade de tensão).

\begin{tabular}{ccccc}
\hline $\mathrm{R}$ & $\begin{array}{c}\operatorname{er}\left(\tilde{\mathrm{A}}_{1}^{(1)}\right) \% \\
\text { MIC }\end{array}$ & $\begin{array}{c}\operatorname{er}\left(\tilde{\mathrm{A}}_{1}^{(1)}\right) \% \\
\text { MFC }\end{array}$ & $\begin{array}{c}\operatorname{er}\left(\tilde{\mathrm{A}}_{1}^{(2)}\right) \% \\
\text { MIC }\end{array}$ & $\begin{array}{c}\operatorname{er}\left(\tilde{\mathrm{A}}_{1}^{(2)}\right) \% \\
\text { MFC }\end{array}$ \\
\hline 0,1 & 0,1669 & 0,2878 & 0,1437 & 0,2067 \\
0,2 & 0,0433 & 0,0788 & 1,0216 & 0,1806 \\
0,3 & 0,166 & 0,1412 & 0,125 & 0,1185 \\
0,4 & 0,1169 & 0,1499 & 0,1922 & 0,1117 \\
0,5 & 0,0759 & 0,1427 & 0,1199 & 0,1322 \\
0,6 & 0,1372 & 0,1271 & 0,1154 & 0,1293 \\
0,7 & 0,1688 & 0,1191 & 0,1455 & 0,126 \\
0,8 & 0,0968 & 0,1199 & 0,1246 & 0,124 \\
0,9 & 0,0628 & 0,1205 & 0,1262 & 0,1279 \\
1,0 & 0,0682 & 0,1159 & 0,1737 & 0,1295 \\
\hline Mín & 0,0433 & 0,0788 & 0,1154 & 0,1117 \\
\hline Média & 0,11028 & 0,14029 & 0,22878 & 0,13864 \\
\hline Máx & 0,1688 & 0,2878 & 1,0216 & 0,2067 \\
\hline
\end{tabular}

Tabela 6.9 - Verificação da independência do caminho (Erro relativo para a taxa de energia disponibilizada para a propagação da fissura).

\begin{tabular}{cccc}
\hline $\mathrm{R}$ & $\operatorname{er}(\mathrm{J}) \%$ & $\operatorname{er}\left(\mathrm{J}_{\mathrm{MIC}}\right) \%$ & $\operatorname{er}\left(\mathrm{J}_{\mathrm{MFC}}\right) \%$ \\
\hline 0,1 & 0,4288 & 0,0234 & 0,4938 \\
0,2 & 1,2544 & 0,9731 & 0,2592 \\
0,3 & 0,254 & 0,2908 & 0,2595 \\
0,4 & 0,2838 & 0,3088 & 0,2614 \\
0,5 & 0,1984 & 0,1957 & 0,2747 \\
0,6 & 0,2597 & 0,2524 & 0,2562 \\
0,7 & 0,3203 & 0,3141 & 0,2450 \\
0,8 & 0,2282 & 0,2212 & 0,2437 \\
0,9 & 0,1852 & 0,1890 & 0,2482 \\
1,0 & 0,2513 & 0,2418 & 0,2452 \\
\hline Mín & 0,1852 & 0,0234 & 0,2437 \\
\hline Média & 0,3664 & 0,3010 & 0,2787 \\
\hline Máx & 1,2544 & 0,9731 & 0,4938 \\
\hline
\end{tabular}

No presente problema, onde as condições de contorno são as componentes de tensão apresentadas nas equações de (6.1) a (6.6), a solução em todo o domínio é regida pelas primeiras parcelas simétrica e anti-simétrica da expansão assintótica. Sob estas condições, todos os métodos implementados apresentaram rendimento satisfatório com respeito à independência do caminho de integração. 


\subsection{Painel Fraturado - tensão constante e fissura horizontal}

O problema anterior foi construído de tal forma que a solução exata fosse representada pelos primeiros termos simétrico e anti-simétrico da expansão assintótica em todo o domínio. Em problemas práticos, essa restrição não se aplica e a expansão assintótica é válida apenas para a vizinhança da ponta da fissura. Nessa seção, apresenta-se o problema de uma chapa sob condições de contorno de Neumman constante ao longo das arestas do domínio que são paralelas a fissura, como apresentado na Figura 6.14.

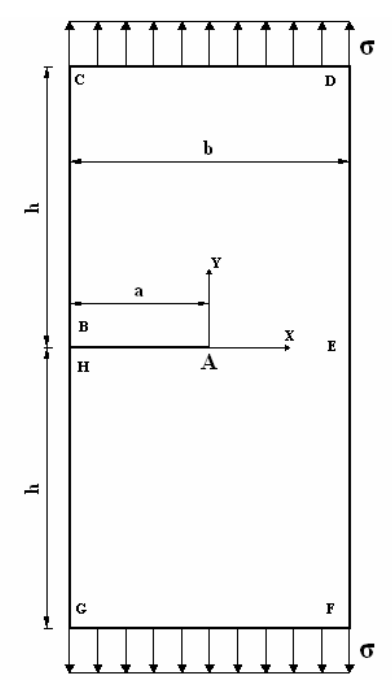

Figura 6.14 - Problema de painel fraturado, sob tensões constantes e com fissura horizontal.

Idealiza-se o problema da seguinte forma:

- Estado Plano de Deformação (EPD);

- Coeficiente de Poisson $v=0,3$;

- $\quad$ Módulo de Young $\mathrm{E}=1,0$;

- Dimensões do domínio $\mathrm{a}=1,0$ e $\mathrm{h}=\mathrm{b}=2,0$;

- Espessura unitária;

- Condições de contorno de Dirichlet: $\mathrm{u}_{\mathrm{xA}}=0,0, \mathrm{u}_{\mathrm{yA}}=0,0$ e $\mathrm{u}_{\mathrm{yE}}=0,0$;

- Condições de contorno de Neumman: $\sigma=1,0$. 
Para o exemplo descrito na Figura 6.14 utilizou-se uma malha geométrica com 3 camadas e taxa de progressão $q=0,15$ na direção da singularidade. A Figura 6.15 apresenta um detalhamento da malha utilizada na análise.

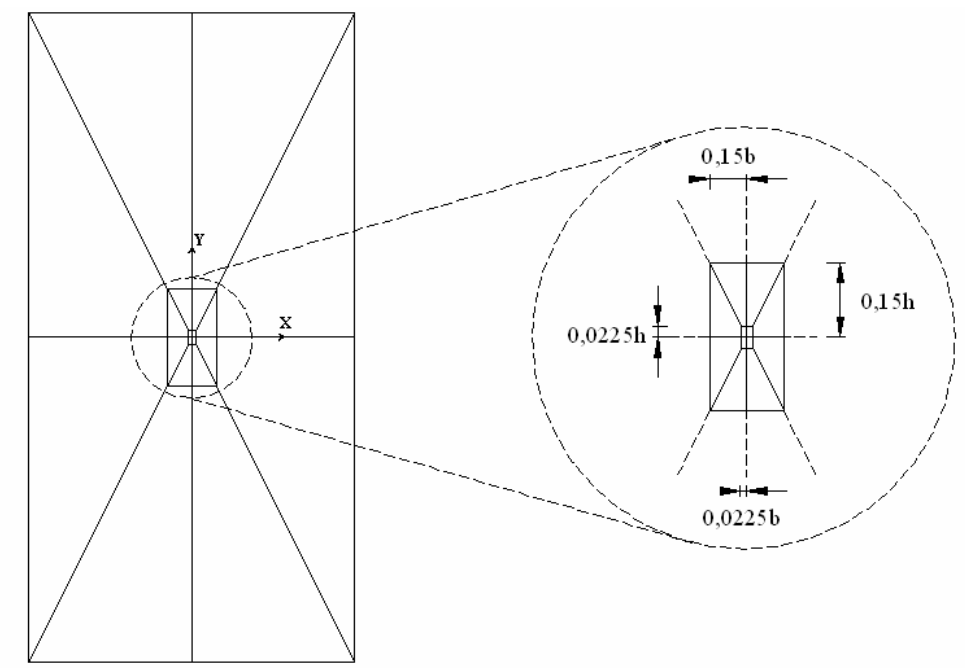

Figura 6.15 - Detalhamento da malha para o problema de painel fraturado - tensão constante e fissura horizontal.

As condições de contorno empregadas na modelagem e a malha utilizada são apresentadas na Figura 6.16.

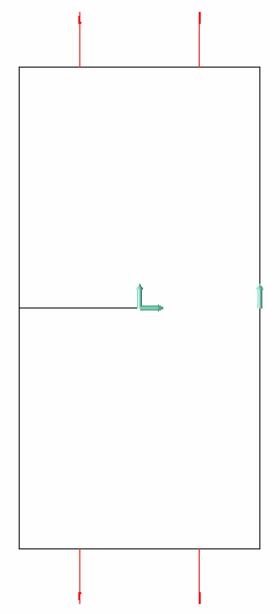

(a)

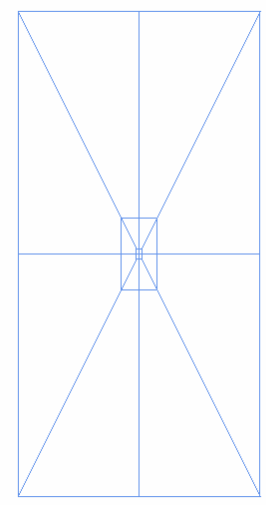

(b)

Figura 6.16 - (a) Condições de contorno aplicadas e (b) malha utilizada na discretização.

A Figura 6.17 apresenta a magnitude do vetor deslocamento e a tensão de von Mises obtidas com o MEFG para o presente problema. 


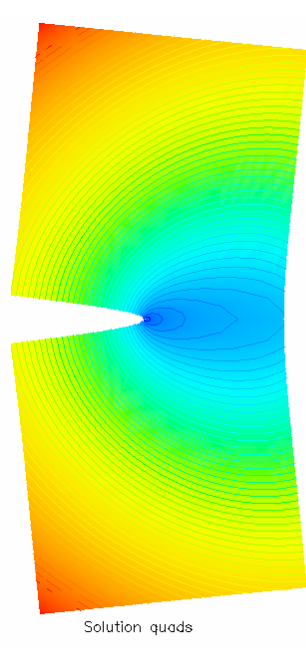

(a)

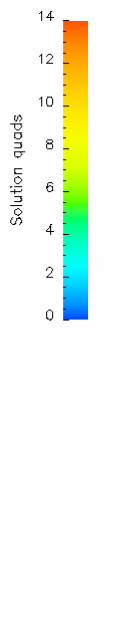

$\mathrm{U}=9,198545583$

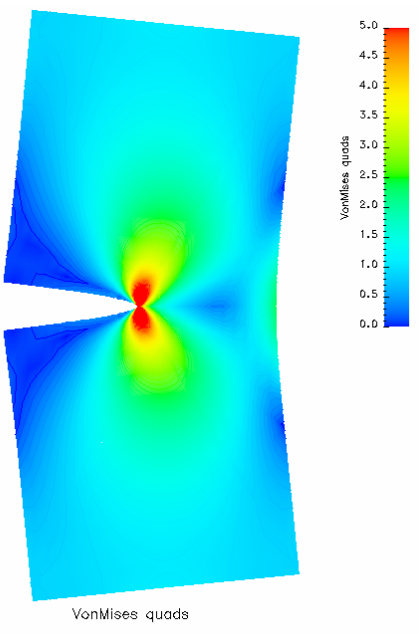

(b)

Figura 6.17 - a) Magnitude do vetor deslocamento e (b) tensão de von Mises para o problema de painel fraturado utilizando tensão constante e fissura horizontal.

Para a energia de deformação foi utilizado o seguinte valor de referência (DUARTE, 1996):

$$
\mathrm{K}_{\mathrm{I}}^{\text {Tada }}=5,009987
$$

dado em (TADA; PARIS; IRWIN, 1973). Este valor de referência possui um erro menor que $0,5 \%$.

A Tabela 6.10 exibe os valores obtidos para energia de deformação e o erro relativo na norma energia e na energia de deformação. Na Figura 6.18 e na Tabela 6.11 apresentam-se a análise da convergência do erro relativo da taxa de energia disponibilizada para a propagação da fissura e dos fatores de intensidade de tensão obtidos com os métodos MIC, MFC e Integral-J, comparando com o erro relativo na norma energia e na energia de deformação. Na presente análise, utilizou-se $\rho=0,05$ para o MIC e $\rho_{1}=0,05$ e $\rho_{2}=0,25$ para o MFC. 


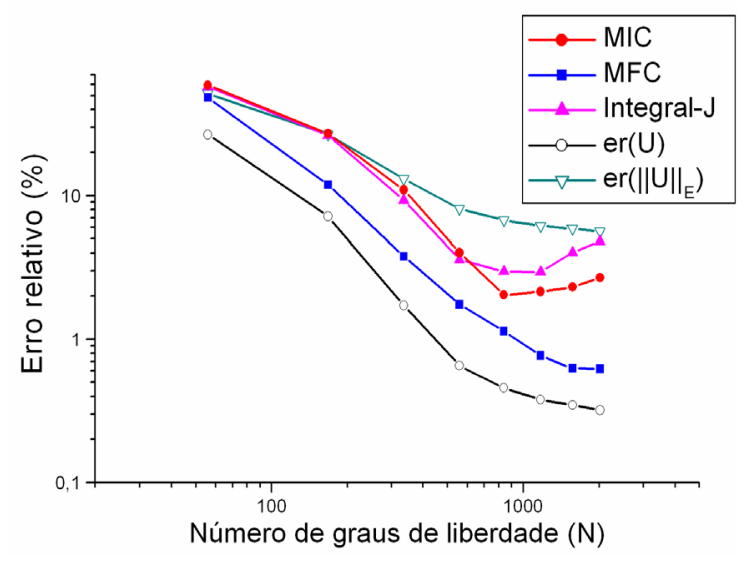

(a)

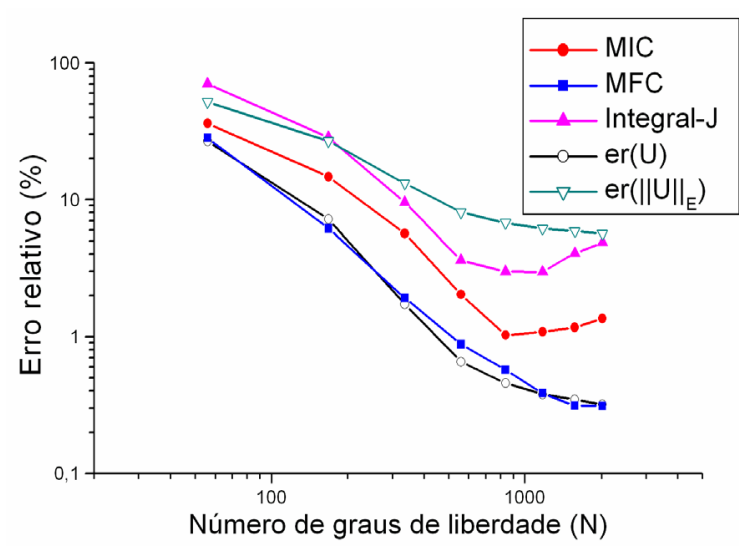

(b)

Figura 6.18 - (a) Taxa de energia disponibilizada - Erro relativo (\%) X Número de graus de liberdade (escala $\log \mathrm{x} \log$ ) e (b) Fator de intensidade de tensão $\mathrm{K}_{\mathrm{I}}-$ Erro relativo (\%) X Número de graus de liberdade (escala log x log).

Tabela 6.10 - Valores de $\operatorname{er}\left(\mathrm{U}_{\mathrm{E}}\right)$ e er $\left(\|\mathrm{U}\|_{\mathrm{E}}\right)$ utilizando o MEFG.

\begin{tabular}{ccccc}
\hline $\mathrm{p}$ & $\mathrm{N}$ & $\mathrm{U}_{\mathrm{MEFG}}$ & $\operatorname{er}\left(\mathrm{U}_{\mathrm{E}}\right) \%$ & $\operatorname{er}\left(\|\mathrm{U}\|_{\mathrm{E}}\right) \%$ \\
\hline 1 & 56 & 6,743007 & 26,6949 & 51,66710753 \\
2 & 168 & 8,539692 & 7,1626 & 26,76303421 \\
3 & 336 & 9,039662 & 1,7273 & 13,14267857 \\
4 & 560 & 9,138391 & 0,654 & 8,087026648 \\
5 & 840 & 9,156458 & 0,4575 & 6,763874629 \\
6 & 1176 & 9,16373 & 0,3785 & 6,152235366 \\
7 & 1568 & 9,16660 & 0,3473 & 5,893216439 \\
8 & 2016 & 9,169151 & 0,3196 & 5,65331761 \\
\hline$\infty$ & 9,198545583 & \\
\hline
\end{tabular}

Tabela 6.11 - Fator de intensidade de tensão e erro relativo (\%) utilizando os métodos de extração MIC, MFC e integral-J.

\begin{tabular}{ccccccc}
\hline $\mathrm{N}$ & $\mathrm{K}_{\mathrm{I}}$ & $\mathrm{er}\left(\mathrm{K}_{\mathrm{I}}\right) \%$ & $\mathrm{~K}_{\mathrm{I}}$ & $\mathrm{er}\left(\mathrm{K}_{\mathrm{I}}\right) \%$ & $\mathrm{~K}_{\mathrm{I}}$ & $\operatorname{er}\left(\mathrm{K}_{\mathrm{I}}\right) \%$ \\
& $\mathrm{~J}$ & $\mathrm{~J}$ & $\mathrm{MIC}$ & $\mathrm{MIC}$ & $\mathrm{MFC}$ & $\mathrm{MFC}$ \\
\hline 56 & 3,254458 & 70,0353 & 3,202852 & 36,0706 & 3,597666 & 28,19 \\
168 & 4,298151 & 28,398 & 4,275436 & 14,6617 & 4,701625 & 6,1549 \\
336 & 4,771129 & 9,528904 & 4,726508 & 5,6582 & 4,913966 & 1,9165 \\
560 & 4,91902 & 3,628904 & 4,908076 & 2,0341 & 4,965869 & 0,8805 \\
840 & 4,934766 & 3,000704 & 4,958552 & 1,0266 & 4,981305 & 0,5724 \\
1176 & 4,935318 & 2,978704 & 4,955724 & 1,083 & 4,990667 & 0,3855 \\
1568 & 4,908003 & 4,068404 & 4,951526 & 1,1668 & 4,994271 & 0,3136 \\
2016 & 4,88781 & 4,874004 & 4,942036 & 1,3562 & 4,994379 & 0,3114 \\
\hline$\infty$ & 5,009983 & $<0,5 \%$ & 5,009983 & $<0,5 \%$ & 5,009983 & $<0,5 \%$ \\
\hline
\end{tabular}

Analisando a Figura 6.18 e a Tabela 6.11 verifica-se que o MFC apresentou resultados mais precisos e de forma mais eficiente. Para se obter um erro relativo menor 
ou igual a 3,0\% utiliza-se o método MFC com ordem polinomial da solução $\mathrm{p}=3$. Com os métodos MIC e Integral-J faz-se necessário o uso de ordens polinomiais maiores, $\mathrm{p}=4$ para o método MIC e $\mathrm{p}=5$ para integral-J. Na presente análise, apenas o MFC exibiu erro menor ou igual a $1,0 \%$.

Observa-se também que o erro relativo para os fatores de intensidade de tensão, obtidos com o MIC e com o MFC, são sempre menores que o erro relativo na norma energia. Também é possível verificar que os métodos MIC e MFC apresentam superconvergência, pois suas taxas de convergência são semelhantes à taxa de convergência da energia de deformação.

A Figura 6.19 e a Tabela 6.12 apresentam a verificação da independência do caminho de integração para os métodos MIC e MFC e integral J. Adota-se a variação dos raios da mesma forma que foi desenvolvida nas verificações para a independência do caminho nos problemas 6.1.1 e 6.1.2.

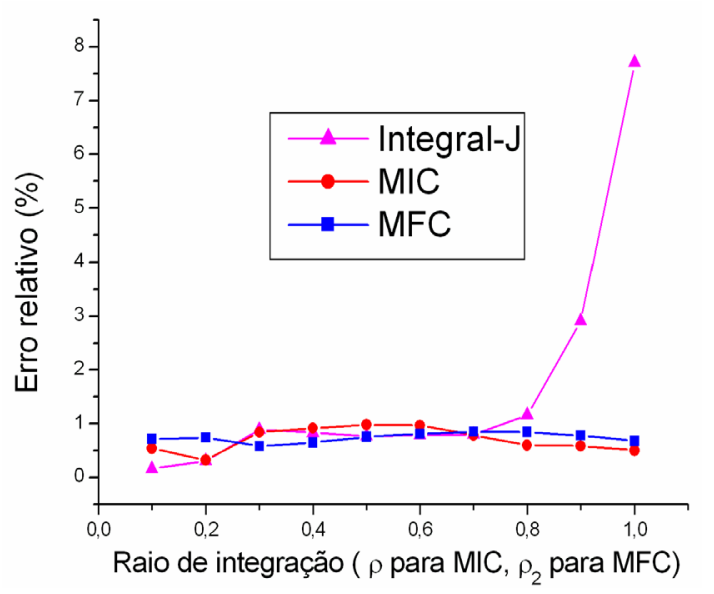

(a)

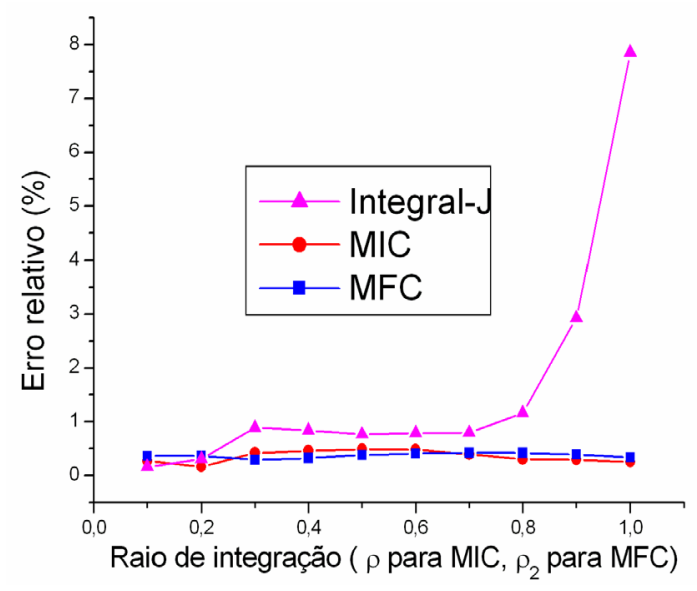

(b)

Figura 6.19 - (a) Taxa de energia disponibilizada - Erro relativo (\%) X raio de integração e (b) Fator de intensidade de tensão $K_{I}-$ Erro relativo (\%) X raio de integração. 
Tabela 6.12 - Verificação da independência do caminho.

\begin{tabular}{cccc}
\hline $\mathrm{R}$ & $\mathrm{er}\left(\mathrm{K}_{\mathrm{I}}\right) \%$ & $\operatorname{er}\left(\mathrm{K}_{\mathrm{I}}\right) \%$ & $\operatorname{er}\left(\mathrm{K}_{\mathrm{I}}\right) \%$ \\
$\mathrm{~J}$ & $\mathrm{MIC}$ & $\mathrm{MFC}$ \\
\hline 0,1 & 0,160736 & 0,2699 & 0,3593 \\
0,2 & 0,307036 & 0,1625 & 0,3702 \\
0,3 & 0,890164 & 0,4211 & 0,2932 \\
0,4 & 0,834664 & 0,4594 & 0,3256 \\
0,5 & 0,767064 & 0,4902 & 0,3783 \\
0,6 & 0,786164 & 0,4839 & 0,4060 \\
0,7 & 0,796464 & 0,3939 & 0,4251 \\
0,8 & 1,162264 & 0,3014 & 0,4221 \\
0,9 & 2,927964 & 0,2928 & 0,3922 \\
1,0 & 7,857464 & 0,2512 & 0,3399 \\
\hline Mín & 0,160736 & 0,1625 & 0,2932 \\
\hline Média & 1,648998 & 0,3526 & 0,3712 \\
\hline Máx & 7,857464 & 0,4902 & 0,4251 \\
\hline
\end{tabular}

Os resultados apresentados aqui mostram que o método da integral-J é sensível à variação do raio quando a solução do domínio não é regida apenas pelos primeiros termos da expansão assintótica. A Integral-J apresenta resultados satisfatórios apenas para a região da vizinhança da ponta da fissura, onde a solução exata do problema é regida pelo primeiro termo simétrico da expansão assintótica (RICE, 1968).

Os métodos MIC e MFC não demonstraram variação significativa no erro relativo da taxa de energia disponibilizada e do fator de intensidade de tensão para os raios distantes da vizinhança da fissura. Sendo assim, esses métodos apresentam-se de forma mais robusta para a extração direta dos fatores de intensidade de tensão.

\subsection{Painel Fraturado - fissura inclinada}

$\mathrm{Na}$ avaliação da robustez dos métodos de extração utiliza-se o problema do painel com fissura inclinada ilustrado na Figura 6.20. Os fatores de intensidade de tensão obtidos nesta análise, utilizando a solução do MEFG, são comparados com os resultados obtidos por Szabó e Babuška (1988) e por Duarte (1996). 


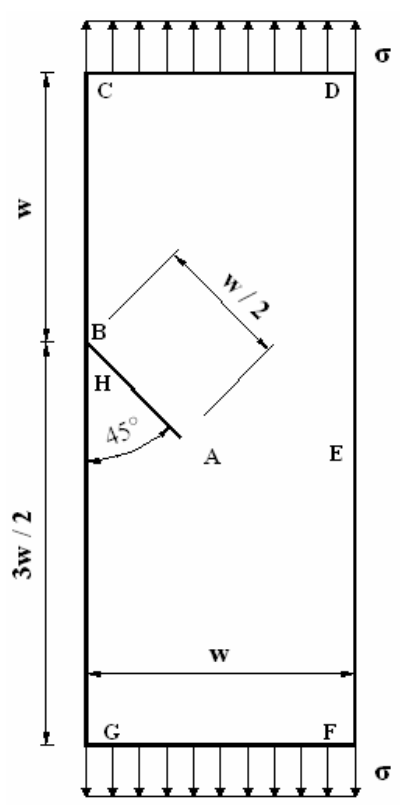

Figura 6.20 - Painel com fissura inclinada - descrição do problema.

Idealize-se o problema de fissura inclinada da seguinte forma:

- Estado plano de tensão;

- Coeficiente de Poisson $v=0,3$;

- Módulo de Young $\mathrm{E}=1,0$;

- Dimensão do domínio $\mathrm{w}=1,0$;

- Espessura unitária;

- Condições de contorno de Dirichlet: $\mathrm{u}_{\mathrm{xA}}=0,0, \mathrm{u}_{\mathrm{yA}}=0,0$ e $\mathrm{u}_{\mathrm{yE}}=0,0$;

- Condições de contorno de Neumman: $\sigma=1,0$.

Nesta análise, utiliza-se uma malha geométrica com 20 elementos quadrilaterais, distribuídos em 3 camadas e fator de progressão geométrica $q=0,15$. A Figura 6.21 apresenta a malha utilizada na análise. 


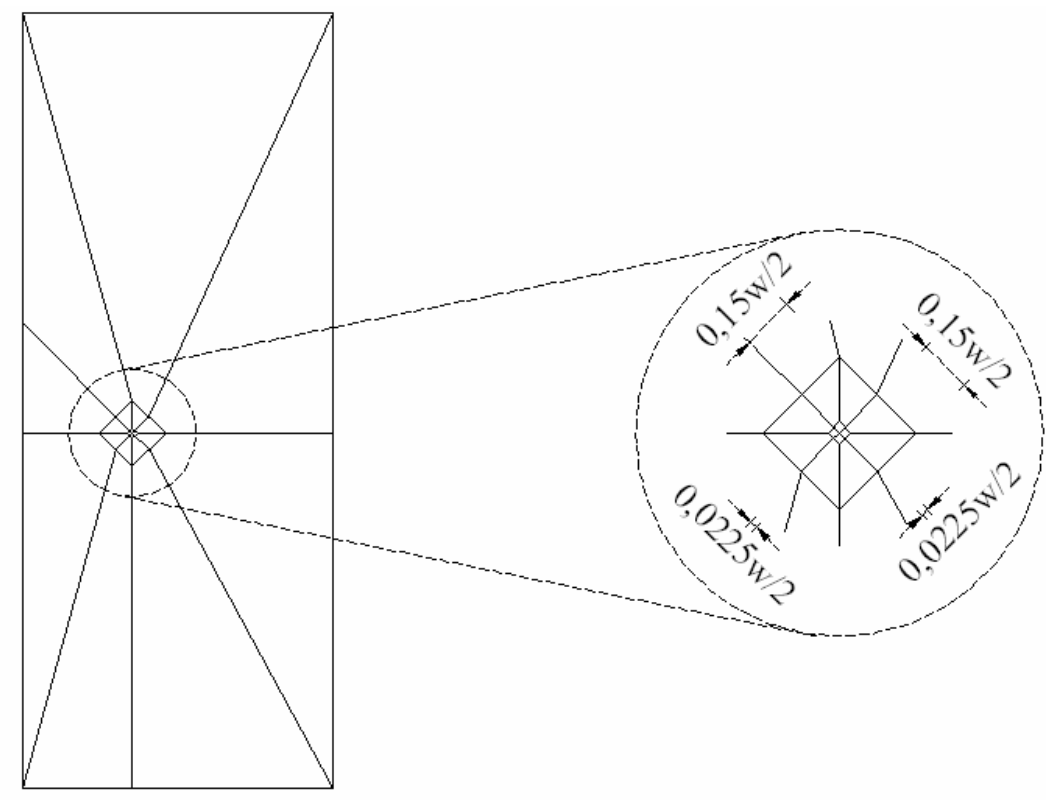

Figura 6.21 - Painel com fissura inclinada - malha utilizada na análise.

A Figura 6.22 apresenta a magnitude do vetor deslocamento e a tensão de von Mises obtidas com o MEFG para o presente problema.

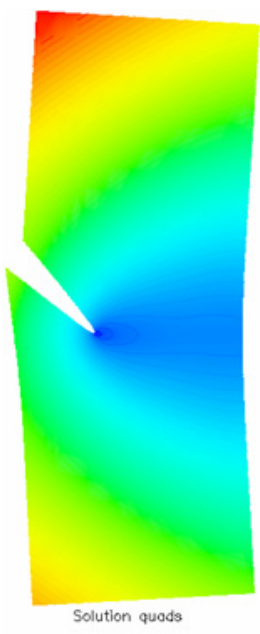

(a)

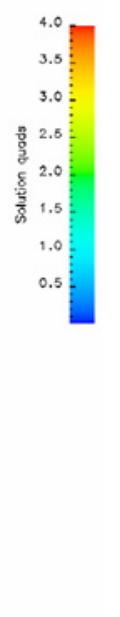

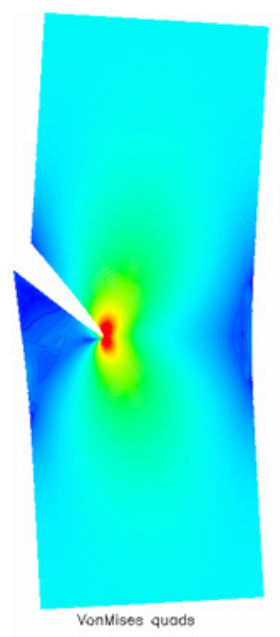

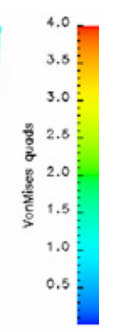

(b)

Figura 6.22 - (a) Magnitude do vetor deslocamento e (b) tensão de von Mises para o problema painel com fissura inclinada.

Os fatores de intensidade de tensão foram obtidos por meio dos métodos MIC e MFC. Os fatores de intensidade de tensão normalizados são definidos na forma:

$$
\widetilde{\mathrm{K}}_{\mathrm{I}} \stackrel{\text { def }}{=} \frac{\mathrm{K}_{\mathrm{I}}}{\sigma \sqrt{2 \pi \mathrm{W}}} ; \quad \widetilde{\mathrm{K}}_{\mathrm{II}} \stackrel{\text { def }}{=} \frac{\mathrm{K}_{\mathrm{II}}}{\sigma \sqrt{2 \pi \mathrm{W}}} .
$$


A Figura 6.23 e a Tabela 6.13 apresentam os fatores de intensidade de tensão normalizados calculados utilizando o MIC e o MFC com a solução do MEFG. Na presente análise, utilizou-se $\rho=0,10$ para o MIC e $\rho_{1}=0,10$ e $\rho_{2}=0,30$ para o MFC. Os resultados obtidos por Szabó e Babuška (1988) com o MIC e com o MFC utilizando a versão p do MEF e por Duarte (1996) utilizando o método das nuvens-hp também são apresentados na Figura 6.23. Szabó e Babuška (1988) utilizaram uma malha geométrica com 3 camadas com fator de progressão igual a 0,15 e elementos curvos. Os valores dos fatores de intensidade de tensão utilizados como referência são:

$$
\widetilde{\mathrm{K}}_{\mathrm{I}}=0,6018 ; \quad \widetilde{\mathrm{K}}_{\mathrm{II}}=-0,2909 .
$$

Estes valores podem ser encontrados em (DUARTE, 1996).

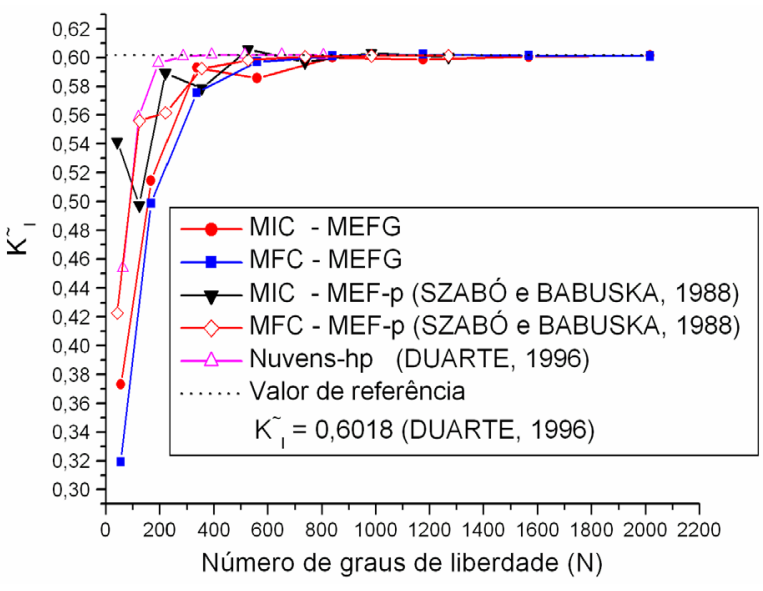

(a)

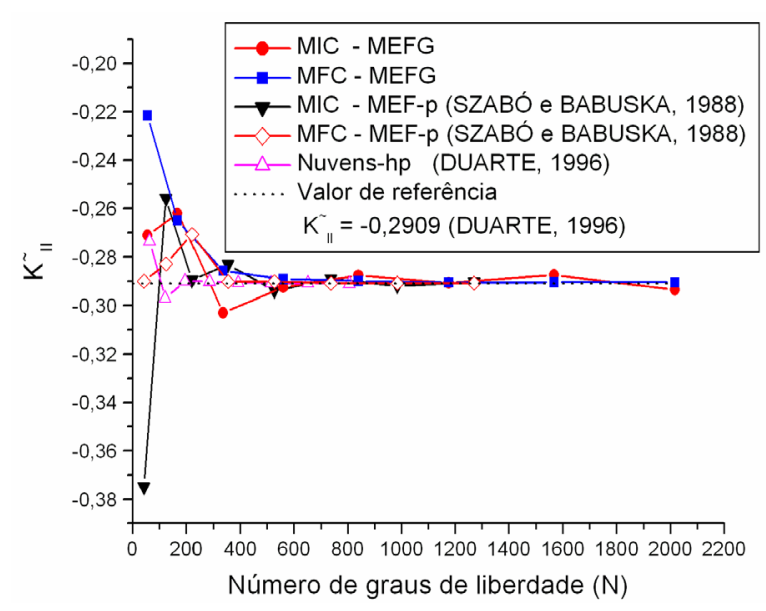

(b)

Figura 6.23 - Fissura inclinada - fator de intensidade de tensão normalizado (a) $\tilde{\mathrm{K}}_{\mathrm{I}}$ e (b) $\tilde{\mathrm{K}}_{\mathrm{II}}$. 
Tabela 6.13 - Fatores de intensidade de tensão normalizados $\tilde{\mathrm{K}}_{\mathrm{I}}$ e $\tilde{\mathrm{K}}_{\mathrm{II}}$ obtido com MIC e MFC utilizando a solução do MEFG.

\begin{tabular}{cccccc}
\hline $\mathrm{p}$ & $\mathrm{N}$ & $\begin{array}{c}\widetilde{\mathrm{K}}_{\mathrm{I}} \\
\mathrm{MIC}\end{array}$ & $\begin{array}{c}\widetilde{\mathrm{K}}_{\mathrm{II}} \\
\mathrm{MIC}\end{array}$ & $\begin{array}{c}\widetilde{\mathrm{K}}_{\mathrm{I}} \\
\mathrm{MFC}\end{array}$ & $\begin{array}{c}\widetilde{\mathrm{K}}_{\mathrm{II}} \\
\mathrm{MFC}\end{array}$ \\
\hline 1 & 56 & 0,37321 & $-0,27116$ & 0,31925 & $-0,22149$ \\
2 & 168 & 0,51432 & $-0,26199$ & 0,4987 & $-0,26502$ \\
3 & 336 & 0,59308 & $-0,30301$ & 0,57553 & $-0,2858$ \\
4 & 560 & 0,58566 & $-0,29261$ & 0,59694 & $-0,28919$ \\
5 & 840 & 0,60008 & $-0,28759$ & 0,60108 & $-0,29003$ \\
6 & 1176 & 0,59853 & $-0,29074$ & 0,60213 & $-0,29064$ \\
7 & 1568 & 0,6007 & $-0,28754$ & 0,6015 & $-0,29054$ \\
8 & 2016 & 0,6016 & $-0,29353$ & 0,60096 & $-0,29061$ \\
\hline
\end{tabular}

Os valores obtidos com os métodos MIC e MFC estão em concordância com os valores encontrados na literatura (DUARTE, 1996; SZABÓ; BABUŠKA, 1988). Os resultados apresentados indicam que os valores calculados possuem forte convergência. Novamente, o método MFC apresentou os melhores resultados e sua convergência é mais monotônica.

A Figura 6.24 apresenta a taxa de energia disponibilizada para a propagação da fissura calculada com os métodos MIC, MFC e integral-J, utilizando a solução do MEFG. O valor de referência para a taxa de energia disponibilizada calculada com os valores apresentados em (6.17) é:

$$
\mathscr{G}=2,8078 \text {. }
$$

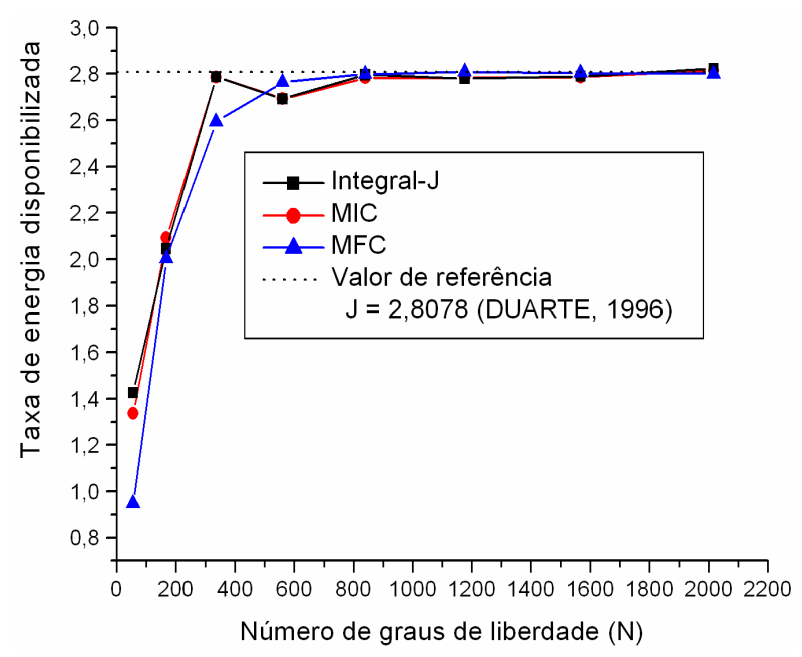

Figura 6.24 - Fissura inclinada - Taxa de energia disponibilizada para a propagação da fissura. 
Os valores para a taxa de energia disponibilizada, obtidos com os métodos MIC, MFC e integral-J, estão em concordância com o valor de referência (6.18). Os resultados obtidos com os métodos MIC e integral-J apresentam comportamento semelhante, isso ocorre em virtude de ambos os métodos possuírem suas formulações baseadas em integrais de contorno em volta da ponta da fissura.

\subsection{Fissura horizontal centrada inserida no domínio}

Nessa seção, apresenta-se o problema de um painel com fissura inserida no domínio sob condição de contorno de Neumman constante ao longo das arestas do domínio que são paralelas à fissura, como apresentado na Figura 6.25. Este é o primeiro problema com fissura inserida completamente no domínio a ser analisado no presente trabalho. A fissura apresentada na Figura 6.25 não possui excentricidade em relação aos eixos de simetria, conseqüentemente os fatores de intensidade de tensão são iguais para as extremidades da fissura. A diferença entre os fatores de intensidade de tensão calculados nos pontos A e B (Figura 6.25) não apresenta variação significativa.

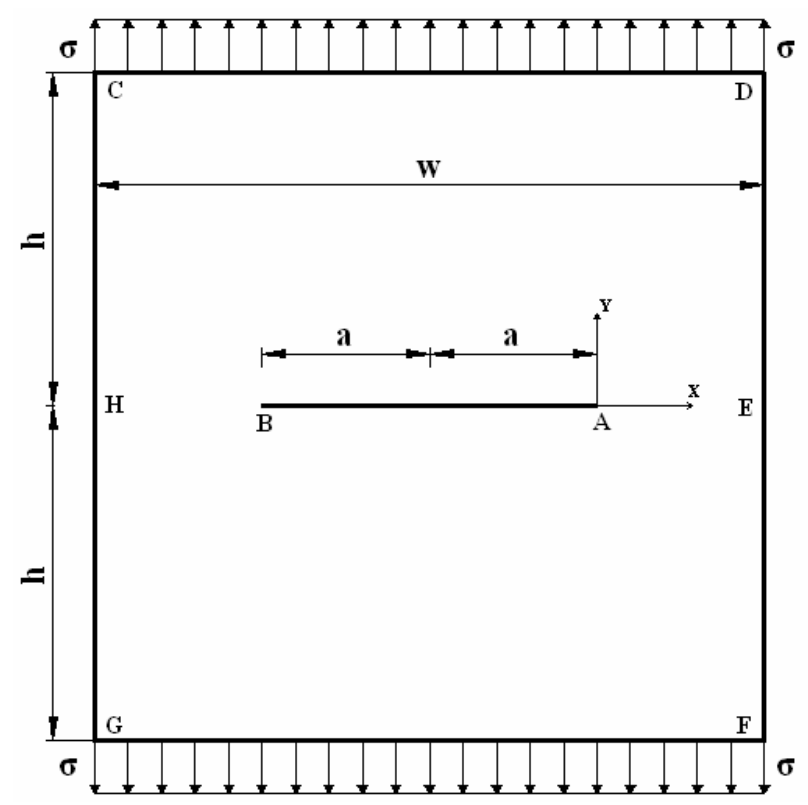

Figura 6.25 - Painel com fissura horizontal centrada - descrição do problema.

Idealiza-se o problema da seguinte forma:

- $\quad$ Estado Plano de Tensão (EPT); 
- Coeficiente de Poisson $v=0,3$;

- Módulo de Young $\mathrm{E}=1,0$;

- Dimensões do domínio $2 \mathrm{~h}=\mathrm{w}=2,0$ e $2 \mathrm{a}=1,0$;

- Espessura unitária;

- Condições de contorno de Dirichlet: $\mathrm{u}_{\mathrm{xH}}=0,0, \mathrm{u}_{\mathrm{yH}}=0,0$ e $\mathrm{u}_{\mathrm{yE}}=0,0$;

- Condições de contorno de Neumman: $\sigma=1,0$.

Na discretização do problema, utilizou-se uma malha geométrica com 3 camadas com progressão geométrica $\mathrm{q}=0,15$ na direção da singularidade em ambas as extremidades da fissura. Um esboço da malha utilizada é apresentado na Figura 6.26.

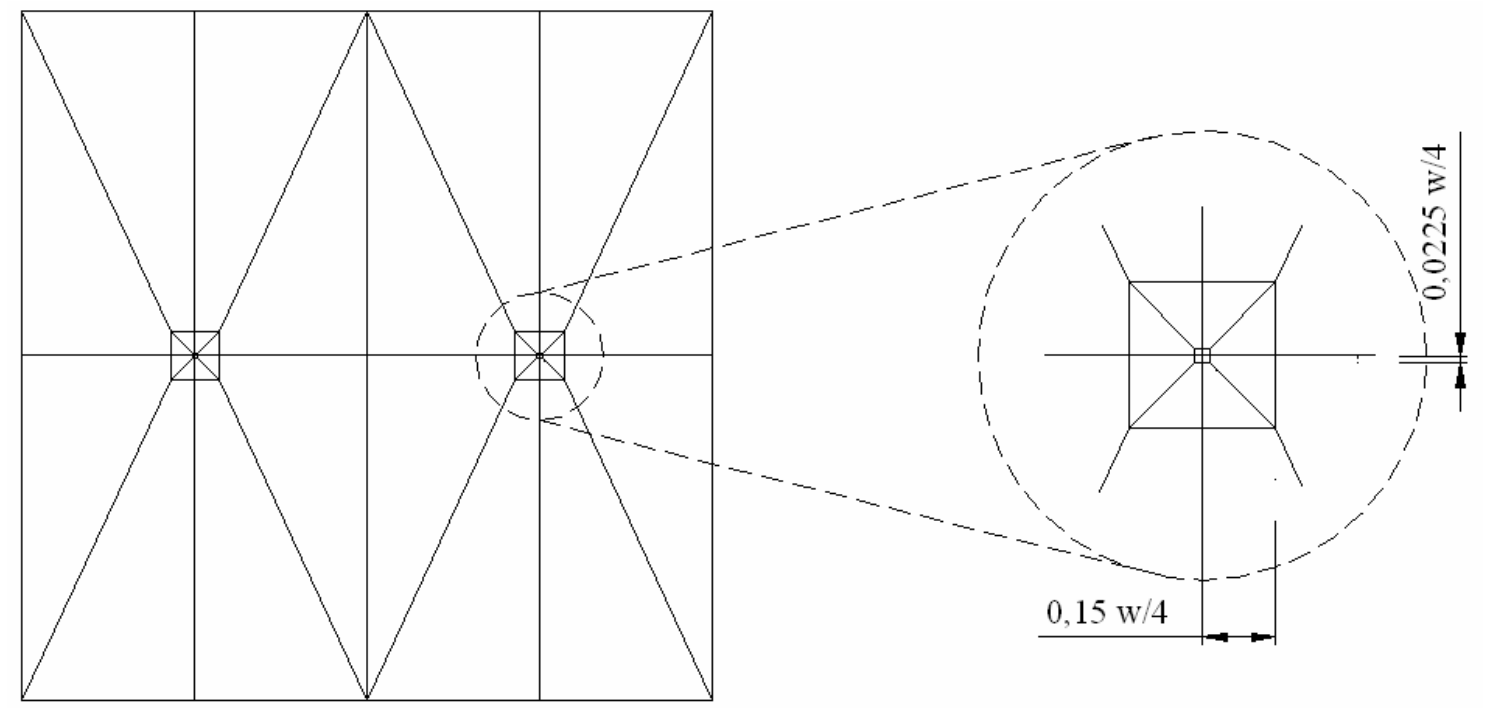

Figura 6.26 - Painel com fissura horizontal centrada - malha utilizada na análise.

A Figura 6.27 apresenta a magnitude do vetor deslocamento e a tensão de von Mises obtidas com o MEFG para o presente problema.

$\mathrm{O}$ valor de referência adotado para o fator de intensidade de tensão normalizado foi:

$$
\tilde{\mathrm{K}}_{\mathrm{I}}=\mathrm{K}_{\mathrm{I}} /(\sigma \sqrt{\mathrm{a} \pi})=1,3341
$$

Este valor de referência pode ser encontrado em (CHANG; MEAR, 1995). 


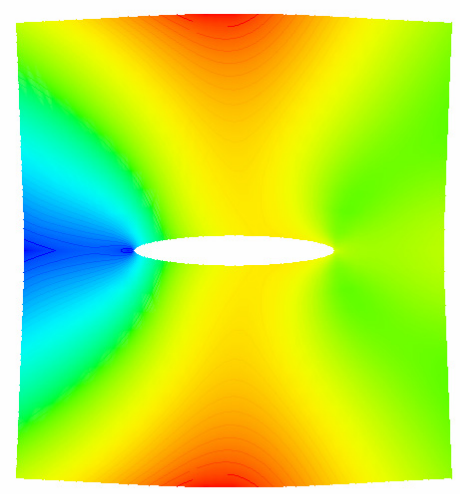

Solution quads

(a)

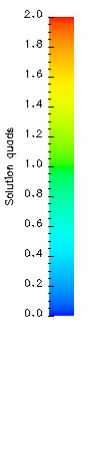

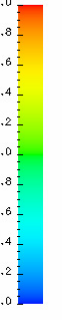

(b)

Figura 6.27 - (a) Magnitude do vetor deslocamento e (b) tensão de von Mises para o problema painel com fissura horizontal centrada.

Na Figura 6.28 (b) e na Tabela 6.14 apresentam-se a análise da convergência do erro relativo dos fatores de intensidade de tensão obtidos com os métodos MIC, MFC e Integral-J. Nessa análise, utilizou-se $\rho=0,10$ para o MIC e $\rho_{1}=0,10$ e $\rho_{2}=0,30$ para o MFC.

Analisando a Figura 6.28 (a) e a Tabela 6.14 verifica-se que o MFC novamente apresentou resultados mais precisos com menor custo computacional. Para se obter um erro relativo menor ou igual a 2,0\% utiliza-se o método MFC com ordem polinomial da solução $p=3$. Com os métodos MIC e Integral-J faz-se necessário o uso de ordens polinomiais maiores, $\mathrm{p}=4$ para o método MIC e $\mathrm{p}=5$ para integral-J. Com o aumento da ordem polinomial, todos os métodos implementados apresentaram valores condizentes com a literatura.

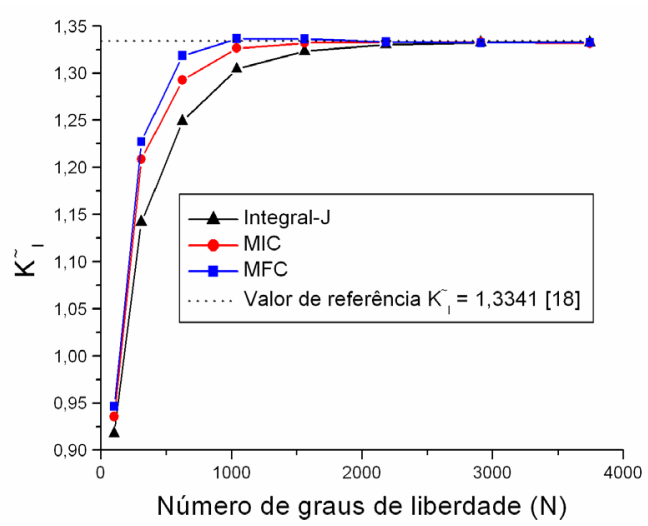

(a)

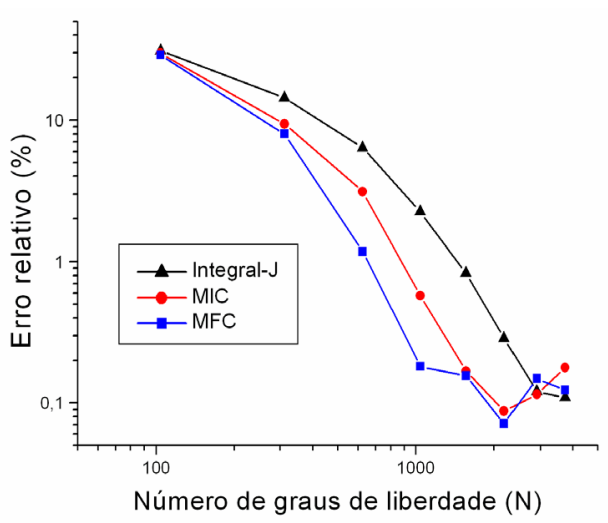

(b)

Figura 6.28 - (a) Fator de intensidade de tensão e (b) erro relativo. 
Tabela 6.14 - Fatores de intensidade de tensão normalizados $\tilde{\mathrm{K}}_{\mathrm{I}}$ e $\tilde{\mathrm{K}}_{\mathrm{II}}$ obtido com MIC e MFC utilizando a solução do MEFG.

\begin{tabular}{ccccc}
\hline $\mathrm{p}$ & $\mathrm{N}$ & $\mathrm{er}\left(\tilde{\mathrm{K}}_{\mathrm{I}}\right) \%$ & $\operatorname{er}\left(\tilde{\mathrm{K}}_{\mathrm{I}}\right) \%$ & $\operatorname{er}\left(\tilde{\mathrm{K}}_{\mathrm{I}}\right) \%$ \\
$\mathrm{~J}$ & 31,2136 & 29,8811 & $\mathrm{MFC}$ \\
\hline 1 & 104 & 14,4367 & 9,4077 & 7,9982 \\
2 & 312 & 6,4003 & 3,1178 & 1,1773 \\
3 & 624 & 2,2490 & 0,5755 & 0,1814 \\
4 & 1040 & 0,8292 & 0,1682 & 0,1558 \\
5 & 1560 & 0,2884 & 0,0878 & 0,0715 \\
6 & 2184 & 0,1207 & 0,1153 & 0,1489 \\
7 & 2912 & 0,1094 & 0,1779 & 0,1237 \\
8 & 3744 & \multicolumn{5}{|}{} \\
\hline$\infty$ & &
\end{tabular}

A Figura 6.29 ilustra a verificação numérica da independência do caminho. Nessa verificação, adota-se a configuração dos domínios de integração da mesma forma que foi empregada nos exemplos anteriores.

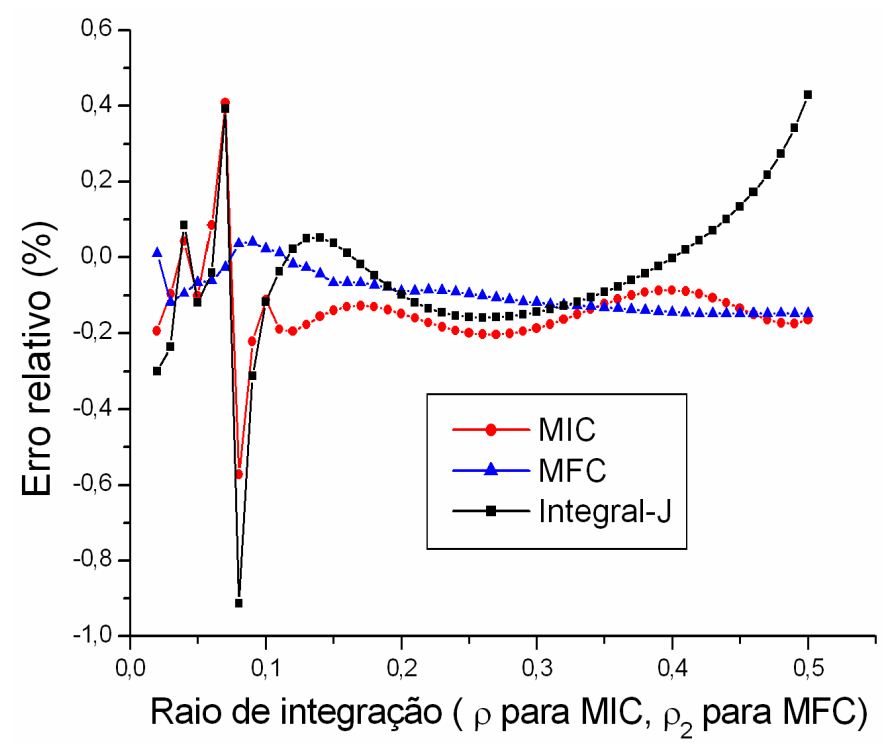

Figura 6.29 - Verificação numérica da independência do caminho de integração.

Na Figura 6.29 é possível observar que os método da Integral-J e da integral de contorno são mais sensíveis a variação do raio quando se utiliza a solução dos elementos que estão muito próximos a ponta da fissura $[\mathrm{r}=0,01 ; 0,10]$. O método da função cutoff não apresenta variação significativa no mesmo intervalo.

No restante do intervalo, os métodos MIC e MFC não apresentam variação significativa, com o erro relativo variando entre $0,1 \%$ e $-0,2 \%$. O método da Integral- 
J é sensível à variação do raio na região distante da extremidade da fissura, onde a solução do problema não é mais regida pelos primeiros termos da expansão assintótica.

\subsection{Fissura inclinada centrada inserida no domínio (com variação do ângulo de inclinação)}

O problema apresentado a seguir serve para verificar a robustez da implementação dos métodos de extração. Considera-se um painel com fissura inclinada inserida no domínio. A Figura 6.30 apresenta um esboço do problema de fissura inclinada com a fissura completamente inserida no domínio.

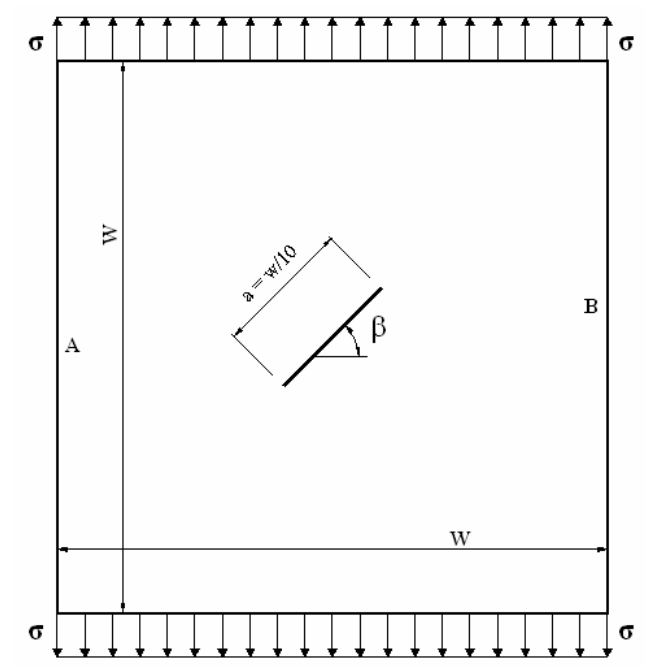

Figura 6.30 - Painel com fissura inclinada centrada - descrição do problema.

Idealiza-se o problema da seguinte forma:

- $\quad$ Estado Plano de Tensão (EPT);

- Coeficiente de Poisson $v=0,3$;

- Módulo de Young E =1;

- Dimensões do domínio $\mathrm{w}=4,0$ e $\mathrm{a}=0,4$;

- Espessura unitária;

- Condições de contorno de Dirichlet: $\mathrm{u}_{\mathrm{xA}}=0,0, \mathrm{u}_{\mathrm{yA}}=0,0$ e $\mathrm{u}_{\mathrm{yB}}=0,0$;

- Condições de contorno de Neumman: $\sigma=1,0$. 
A Figura 6.31 apresenta um esboço da discretização utilizada na análise do problema. A malha possui 54 elementos e uma taxa de progressão geométrica $q=0.15$ nos elementos próximos ao pontos de singularidade.

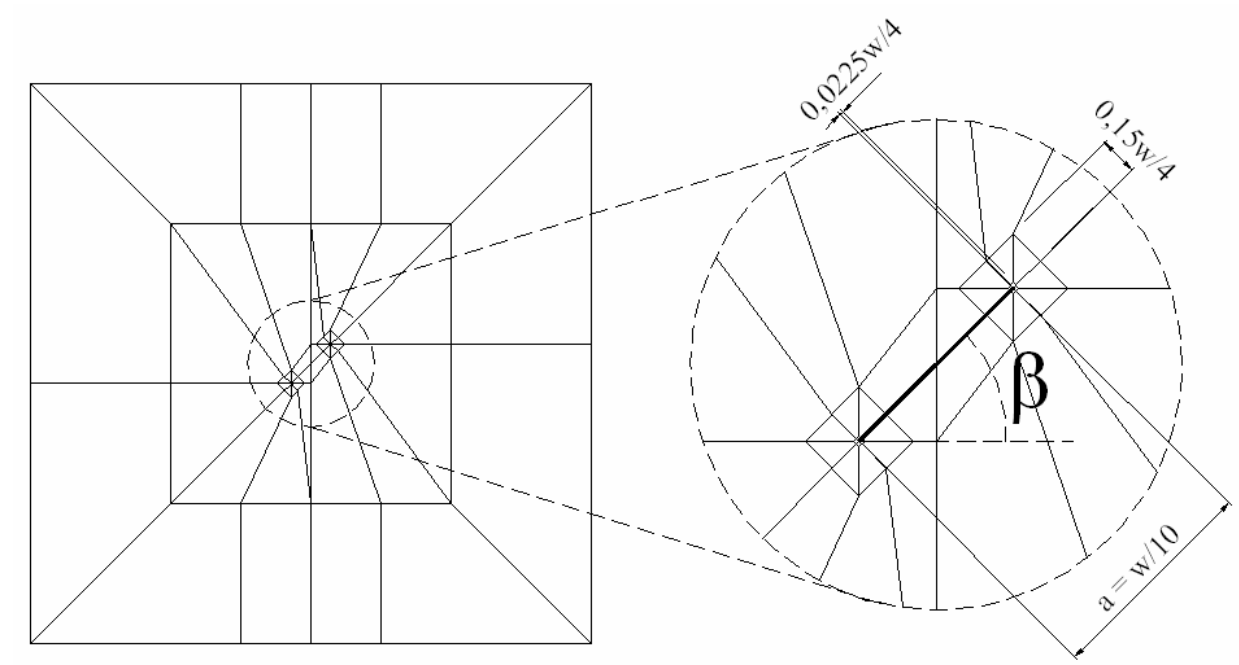

Figura 6.31 - Painel com fissura inclinada centrada - malha utilizada na análise.

Na configuração dos domínios de integração, utilizou-se $\rho=0,02$ para o MIC e $\rho_{1}=0,02$ e $\rho_{2}=0,30$ para o MFC. A Figura 6.32 e a Tabela 6.15 apresentam os fatores de intensidade de tensão variando com ângulo de inclinação da fissura $(\beta)$. Nessa análise, utilizou-se o enriquecimento polinomial da solução do MEFG $p=7$. Na presente simulação, utilizou-se uma razão entre a aresta do domínio e a aresta da fissura relativamente grande $(\mathrm{w} / \mathrm{a}=10)$, sendo assim, é possível validar a solução numérica utilizando os valores analíticos para uma fissura em domínio infinito (TADA; PARIS; IRWIN, 1973).

$$
\mathrm{K}_{\mathrm{I}}=\sigma \sqrt{\pi \mathrm{a} / 2} \cos ^{2}(\beta) ; \quad \mathrm{K}_{\mathrm{II}}=\sigma \sqrt{\pi \mathrm{a} / 2} \operatorname{sen}(\beta) \cos (\beta)
$$




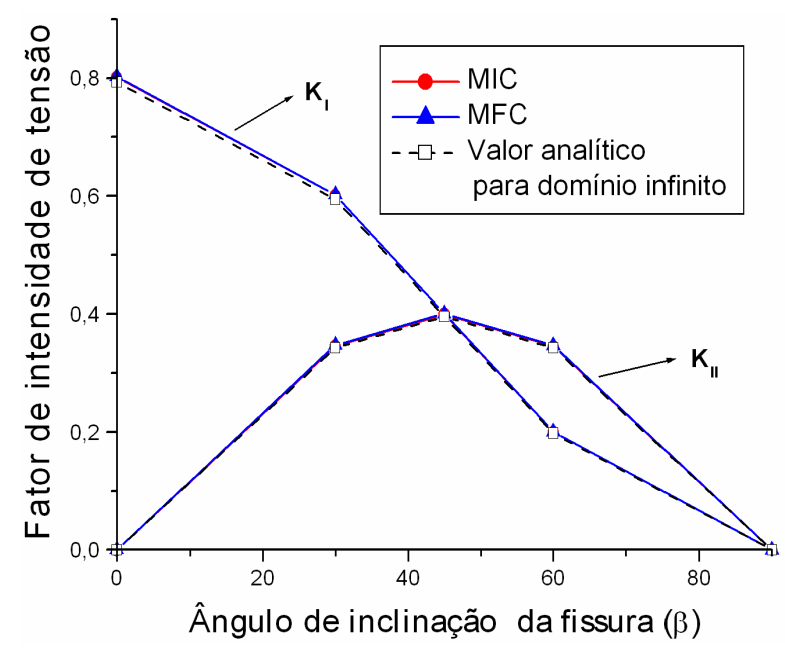

Figura 6.32 - Fator de intensidade de tensão versus ângulo de Inclinação $\beta$.

Tabela 6.15 - Valores $\mathrm{K}_{\mathrm{I}}$ e $\mathrm{K}_{\mathrm{II}}$ para todos os ângulos $\beta$ analisados, utilizando enriquecimento $\mathrm{p}=7$.

\begin{tabular}{ccccccc}
\hline$\beta$ & $\mathrm{K}_{\mathrm{I}}$ & $\mathrm{K}_{\mathrm{I}}$ & $\mathrm{K}_{\mathrm{I}}$ & $\mathrm{K}_{\mathrm{II}}$ & $\mathrm{K}_{\mathrm{II}}$ & $\mathrm{K}_{\mathrm{II}}$ \\
& Analítico & $\mathrm{MIC}$ & $\mathrm{MFC}$ & Analítico & $\mathrm{MIC}$ & MFC \\
\hline $0^{\circ}$ & 0,793 & 0,8019 & 0,80325 & 0 & $-3,1333 \mathrm{E}-4$ & $2,2560 \mathrm{E}-4$ \\
$30^{\circ}$ & 0,594 & 0,60159 & 0,60184 & 0,343 & 0,34629 & 0,3475 \\
$45^{\circ}$ & 0,396 & 0,40065 & 0,40074 & 0,396 & 0,39986 & 0,40117 \\
$60^{\circ}$ & 0,198 & 0,20037 & 0,200254 & 0,343 & 0,346061 & 0,346695 \\
$90^{\circ}$ & 0 & 0 & 0 & 0 & 0 & 0 \\
\hline
\end{tabular}

Da Figura 6.33 à Figura 6.37 apresenta-se a convergência dos fatores de intensidade de tensão com o enriquecimento polinomial da solução do MEFG para cada inclinação de fissura analisada. Da Tabela 6.16 à Tabela 6.19 apresenta-se o erro relativo para os fatores de intensidade de tensão $K_{I}$ e $K_{\text {II }}$ para cada ângulo $\beta$ analisado. Em todas as análises apresentadas nesta seção, utilizou-se $\rho=0,02$ para o MIC e $\rho_{1}=0,02$ e $\rho_{2}=0,30$ para o MFC. 


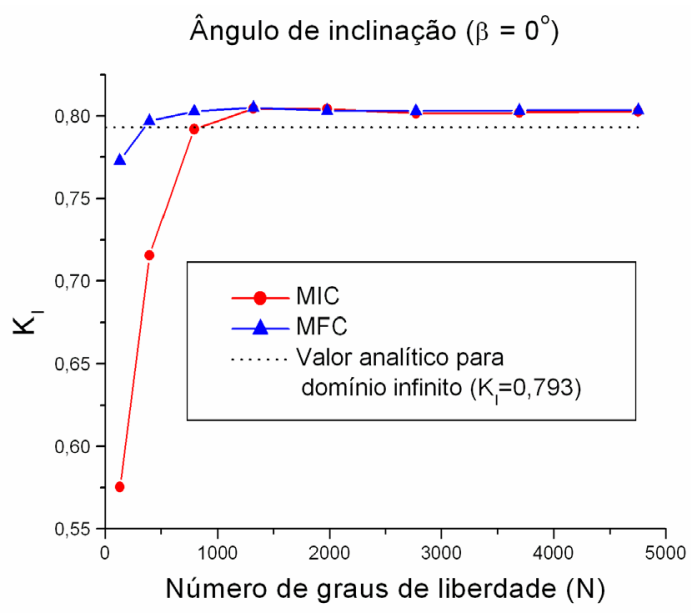

(a)

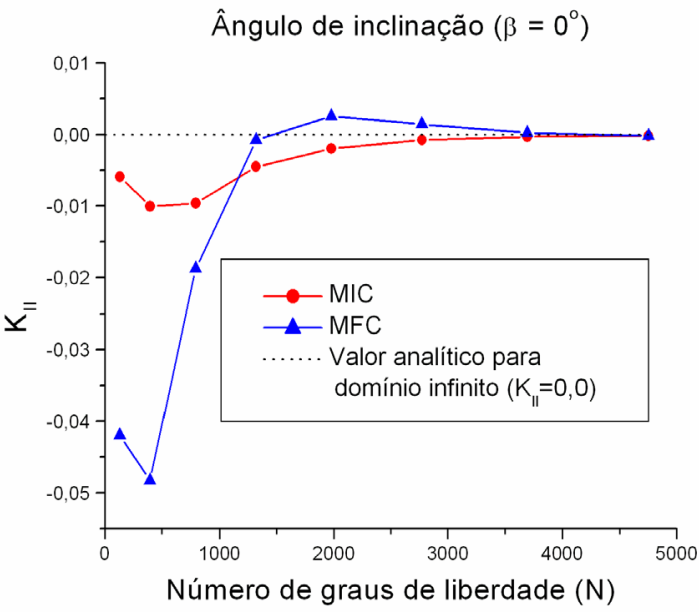

(b)

Figura 6.33 - Fatores de intensidade de tensão $K_{I}$ (a) e $K_{\text {II }}$ (b) para $\beta=0^{\circ}$.

Tabela 6.16 - Erro relativo para os fatores de intensidade de tensão $\mathrm{K}_{\mathrm{I}}$ e $\mathrm{K}_{\text {II }}$ para $\beta=0^{\circ}$, utilizando os métodos MIC e MFC.

\begin{tabular}{cccccc}
\hline $\mathrm{p}$ & $\mathrm{N}$ & $\begin{array}{c}\mathrm{er}\left(\mathrm{K}_{\mathrm{I}}\right) \% \\
\mathrm{MIC}\end{array}$ & $\begin{array}{c}\operatorname{er}\left(\mathrm{K}_{\mathrm{II}}\right) \% \\
\mathrm{MIC}\end{array}$ & $\begin{array}{c}\operatorname{er}\left(\mathrm{K}_{\mathrm{I}}\right) \% \\
\mathrm{MFC}\end{array}$ & $\begin{array}{c}\operatorname{er}\left(\mathrm{K}_{\mathrm{II}}\right) \% \\
\mathrm{MFC}\end{array}$ \\
\hline 1 & 132 & 27,39397 & 0,526818 & 5,753328 & 4,490997 \\
2 & 396 & 9,726222 & 0,896663 & 0,590472 & 5,699117 \\
3 & 792 & 0,075549 & 0,859097 & 1,024325 & 3,241754 \\
4 & 1320 & 1,503161 & 0,402045 & 1,811913 & 1,073686 \\
5 & 1980 & 1,455972 & 0,17732 & 1,576848 & 0,129527 \\
6 & 2772 & 1,138009 & 0,068647 & 1,411558 & 0,097413 \\
7 & 3696 & 1,180783 & 0,027951 & 1,402347 & 0,072168 \\
8 & 4752 & 1,274531 & 0,017218 & 1,427582 & 0,015879 \\
\hline
\end{tabular}

Ângulo de inclinação $\left(\beta=30^{\circ}\right)$

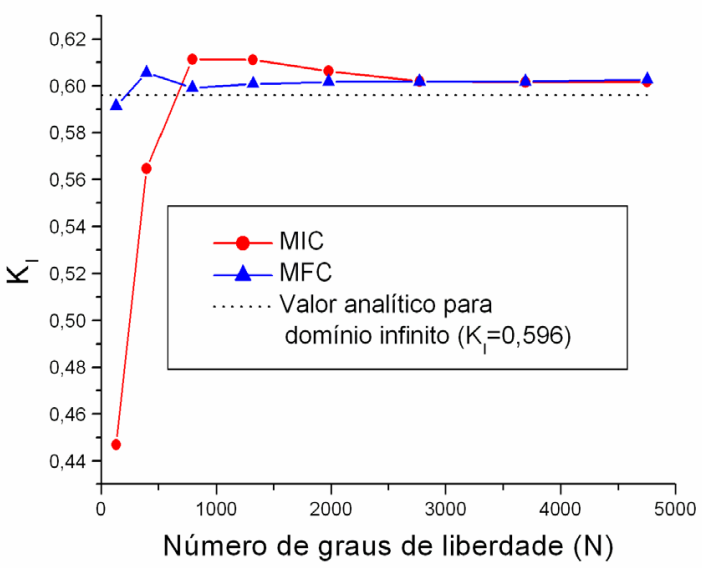

(a)

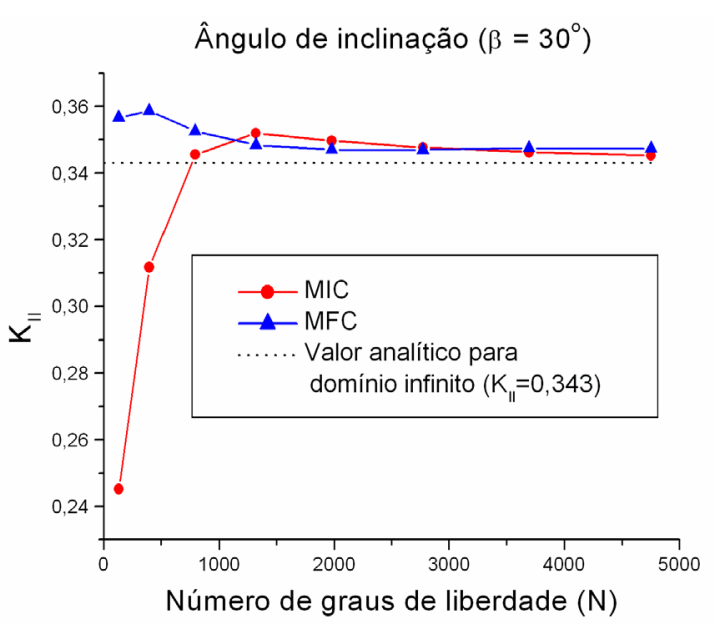

(b)

Figura 6.34 - Fatores de intensidade de tensão $K_{I}$ (a) e $K_{\text {II }}$ (b) para $\beta=30^{\circ}$. 
Tabela 6.17 - Erro relativo para os fatores de intensidade de tensão $\mathrm{K}_{\mathrm{I}}$ e $\mathrm{K}_{\mathrm{II}}$ para $\beta=30^{\circ}$, utilizando os métodos MIC e MFC.

\begin{tabular}{cccccc}
\hline $\mathrm{p}$ & $\mathrm{N}$ & $\begin{array}{c}\mathrm{er}\left(\mathrm{K}_{\mathrm{I}}\right) \% \\
\mathrm{MIC}\end{array}$ & $\begin{array}{c}\operatorname{er}\left(\mathrm{K}_{\mathrm{II}}\right) \% \\
\mathrm{MIC}\end{array}$ & $\begin{array}{c}\mathrm{er}\left(\mathrm{K}_{\mathrm{I}}\right) \% \\
\mathrm{MFC}\end{array}$ & $\begin{array}{c}\operatorname{er}\left(\mathrm{K}_{\mathrm{II}}\right) \% \\
\mathrm{MFC}\end{array}$ \\
\hline 1 & 132 & 24,77108 & 28,50648 & 0,465442 & 3,983007 \\
2 & 396 & 4,951079 & 9,113336 & 1,927151 & 4,576369 \\
3 & 792 & 2,895832 & 0,746554 & 0,845822 & 2,791899 \\
4 & 1320 & 2,876425 & 2,621636 & 1,128917 & 1,585445 \\
5 & 1980 & 2,04908 & 1,952277 & 1,283332 & 1,179153 \\
6 & 2772 & 1,331007 & 1,358915 & 1,304849 & 1,130193 \\
7 & 3696 & 1,25633 & 0,952623 & 1,29852 & 1,303379 \\
8 & 4752 & 1,27616 & 0,656673 & 1,433528 & 1,248573 \\
\hline
\end{tabular}

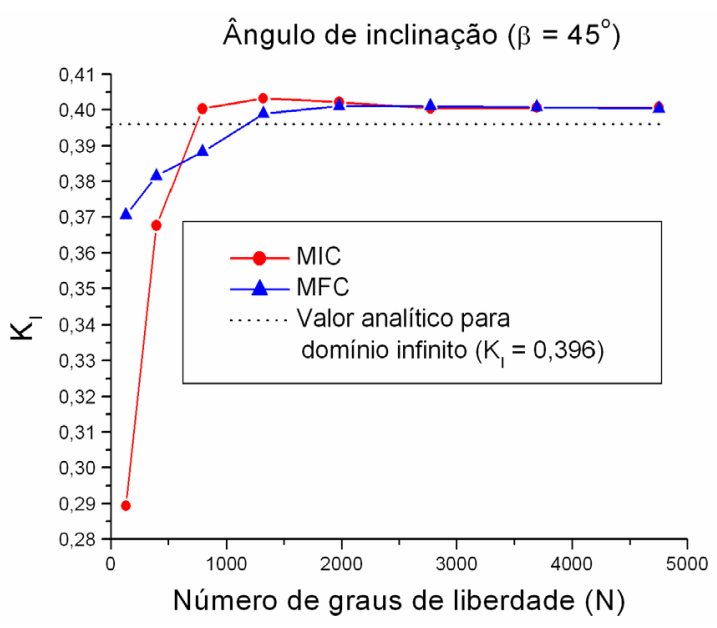

(a)

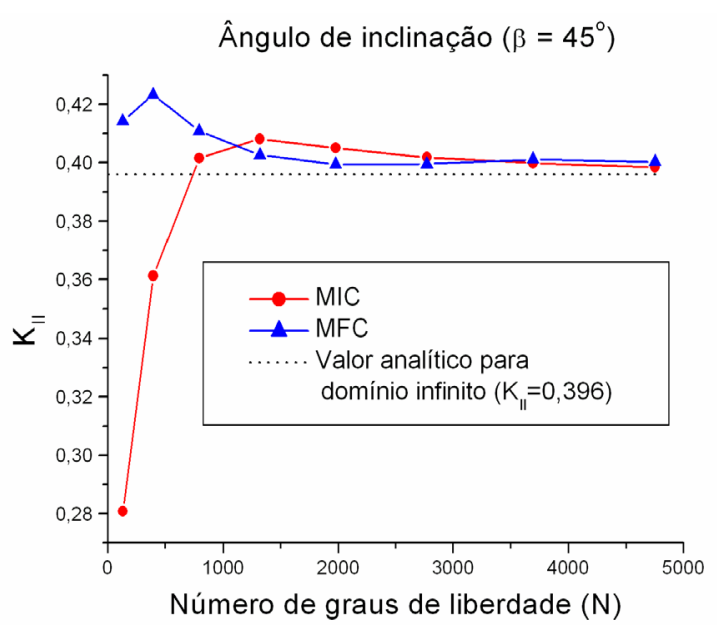

(b)

Figura 6.35 - Fatores de intensidade de tensão $K_{I}$ (a) e $K_{\text {II }}$ (b) para $\beta=45^{\circ}$.

Tabela 6.18 - Erro relativo para os fatores de intensidade de tensão $K_{I}$ e $K_{I I}$ para $\beta=45^{\circ}$, utilizando os métodos MIC e MFC.

\begin{tabular}{cccccc}
\hline $\mathrm{p}$ & $\mathrm{N}$ & $\begin{array}{c}\mathrm{er}\left(\mathrm{K}_{\mathrm{I}}\right) \% \\
\mathrm{MIC}\end{array}$ & $\begin{array}{c}\mathrm{er}\left(\mathrm{K}_{\mathrm{II}}\right) \% \\
\mathrm{MIC}\end{array}$ & $\begin{array}{c}\mathrm{er}\left(\mathrm{K}_{\mathrm{I}}\right) \% \\
\mathrm{MFC}\end{array}$ & $\begin{array}{c}\operatorname{er}\left(\mathrm{K}_{\mathrm{II}}\right) \% \\
\mathrm{MFC}\end{array}$ \\
\hline 1 & 132 & 27,08271 & 29,22782 & 6,617632 & 4,378895 \\
2 & 396 & 7,343407 & 8,951607 & 3,860447 & 6,668654 \\
3 & 792 & 0,887092 & 1,187129 & 2,149286 & 3,524262 \\
4 & 1320 & 1,609077 & 2,857232 & 0,523889 & 1,454952 \\
5 & 1980 & 1,354519 & 2,063238 & 1,058271 & 0,637587 \\
6 & 2772 & 0,919938 & 1,2484 & 1,049428 & 0,678013 \\
7 & 3696 & 0,961627 & 0,763287 & 0,984999 & 1,093644 \\
8 & 4752 & 0,988157 & 0,401979 & 0,894672 & 0,861826 \\
\hline
\end{tabular}




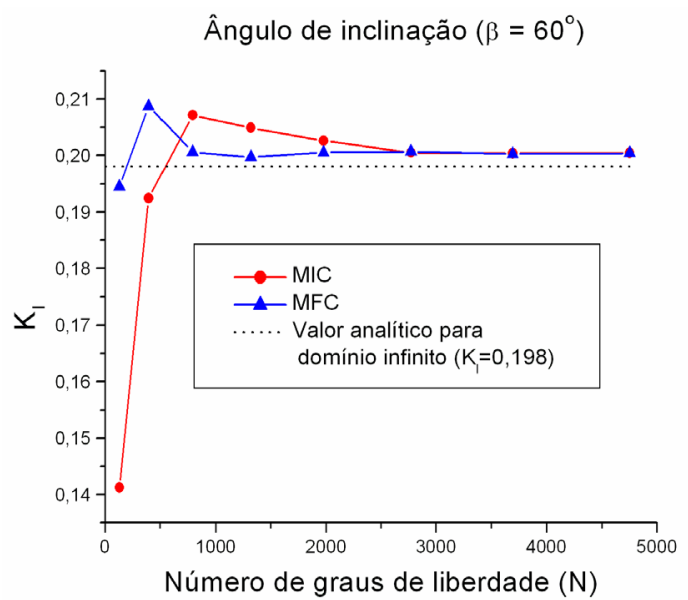

(a)

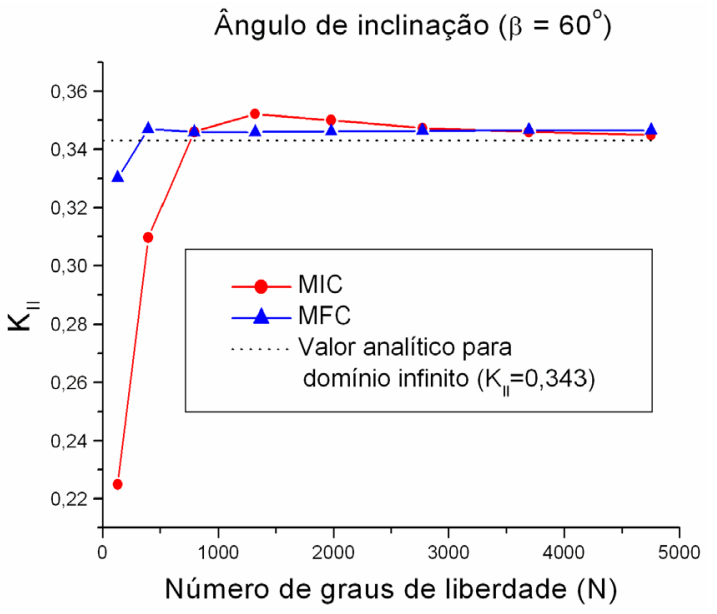

(b)

Figura 6.36 - Fatores de intensidade de tensão $K_{I}$ (a) e $K_{\text {II }}$ (b) para $\beta=60^{\circ}$.

Tabela 6.19 - Erro relativo para os fatores de intensidade de tensão $K_{I}$ e $K_{\text {II }}$ para $\beta=60^{\circ}$, utilizando os métodos MIC e MFC.

\begin{tabular}{cccccc}
\hline $\mathrm{p}$ & $\mathrm{N}$ & $\begin{array}{c}\mathrm{er}\left(\mathrm{K}_{\mathrm{I}}\right) \% \\
\mathrm{MIC}\end{array}$ & $\begin{array}{c}\operatorname{er}\left(\mathrm{K}_{\mathrm{II}}\right) \% \\
\mathrm{MIC}\end{array}$ & $\begin{array}{c}\operatorname{er}\left(\mathrm{K}_{\mathrm{I}}\right) \% \\
\mathrm{MFC}\end{array}$ & $\begin{array}{c}\operatorname{er}\left(\mathrm{K}_{\text {II }}\right) \% \\
\mathrm{MFC}\end{array}$ \\
\hline 1 & 132 & 28,82202 & 34,43227 & 1,981027 & 3,724931 \\
2 & 396 & 3,009671 & 9,741394 & 5,171093 & 1,168292 \\
3 & 792 & 4,415612 & 0,884931 & 1,119518 & 0,849948 \\
4 & 1320 & 3,285163 & 2,674594 & 0,640728 & 0,827792 \\
5 & 1980 & 2,10885 & 2,054815 & 1,067103 & 0,914375 \\
6 & 2772 & 1,049968 & 1,243505 & 1,154797 & 0,966557 \\
7 & 3696 & 0,984449 & 0,884931 & 0,925986 & 1,069757 \\
8 & 4752 & 1,028296 & 0,538309 & 1,02124 & 1,042353 \\
\hline
\end{tabular}

Ângulo de inclinação $\left(\beta=90^{\circ}\right)$

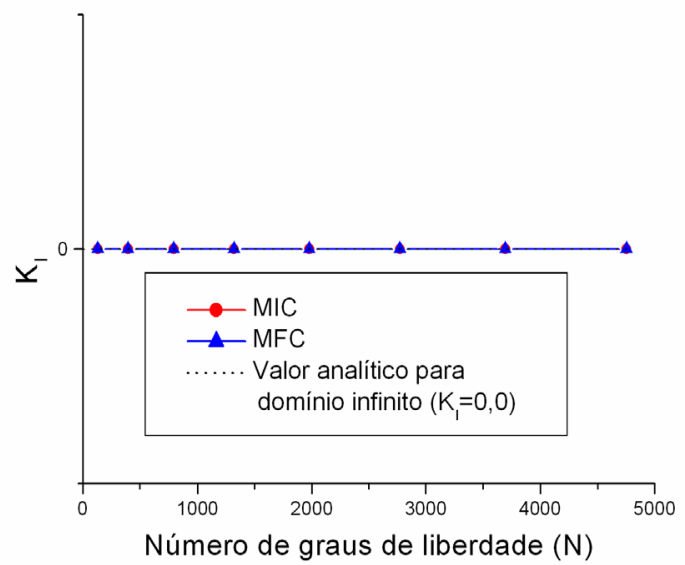

(a)

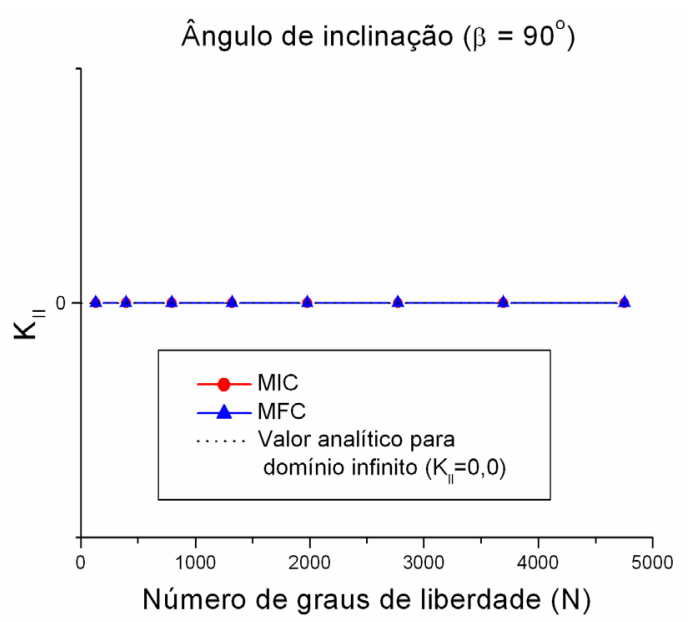

(b)

Figura 6.37 - Fatores de intensidade de tensão $K_{I}$ (a) e $K_{\text {II }}$ (b) para $\beta=90^{\circ}$. 
Nota-se que os fatores de intensidade de tensão, calculados numericamente, convergem para um valor aproximadamente $1 \%$ maior que o valor analítico para domínio infinito. Atribui-se tal comportamento ao efeito causado pela restrição de tamanho do domínio de análise.

Outra maneira de validar os resultados obtidos com os método MIC e MFC neste exemplo é a comparação entre a convergência da taxa de energia disponibilizada para a propagação da fissura com o aumento da ordem polinomial do enriquecimento, para os métodos MIC e MFC, e a taxa de energia disponibilizada obtida com a Integral-J. A Figura 6.40 ilustra essa análise. Para todas as inclinações analisadas, é possível observar que o resultados obtidos com os métodos MFC e MIC são condizentes com os valores obtidos para a Integral-J.

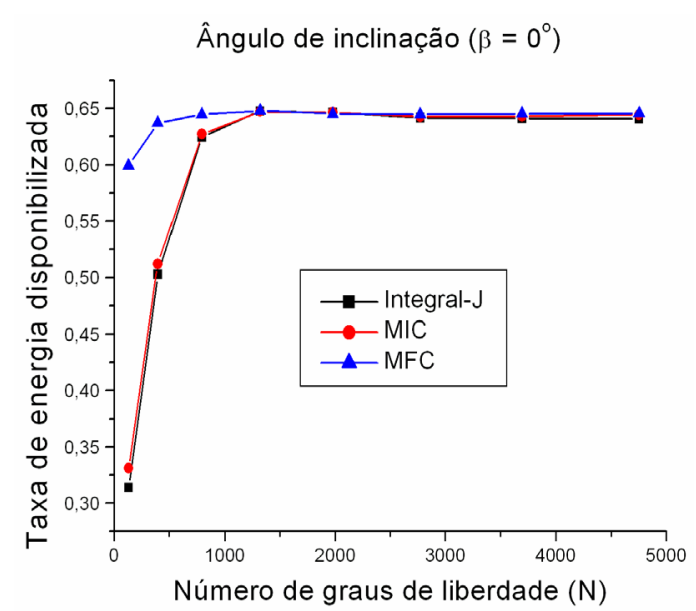

(a)

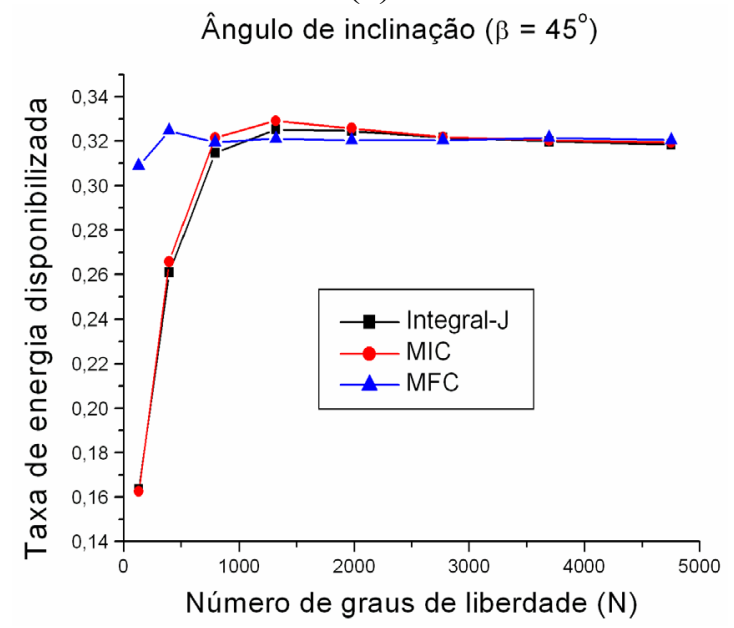

(c)

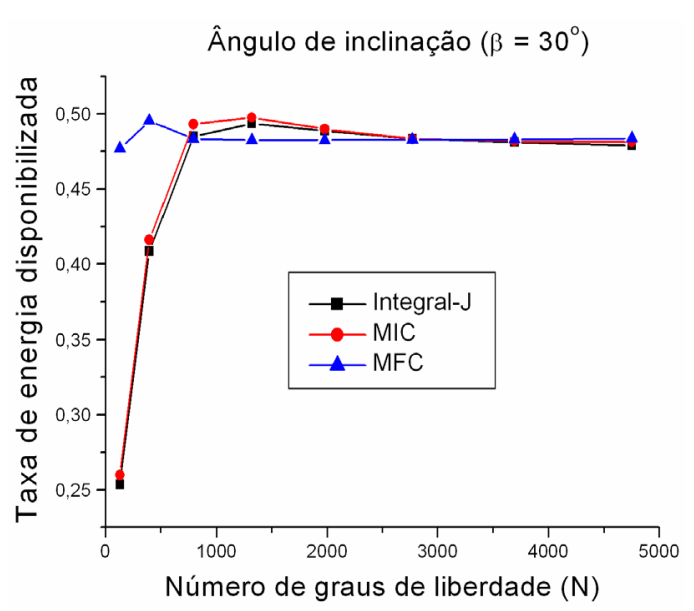

(b)

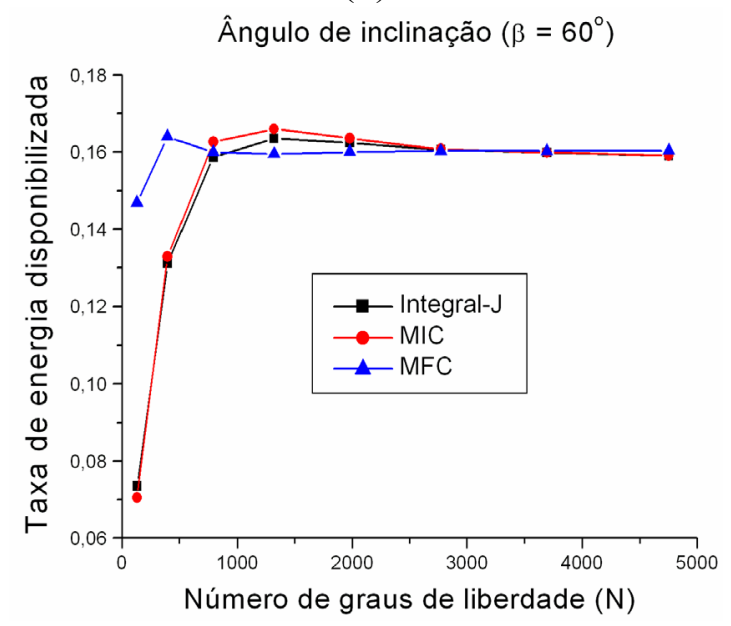

(d)

Figura 6.38 - Taxa de energia disponibilizada para a propagação da fissura para $0^{\circ}$ (a), $30^{\circ}$ (b), $45^{\circ}$ (c) e $60^{\circ}$ (d). 


\subsection{Fissura inclinada excêntrica inserida no domínio}

O problema apresentado a seguir serve para verificar a robustez da implementação dos métodos de extração e a versatilidade da implementação utilizando os recursos da programação orientada a objetos. Considera-se o problema de um painel com fissura inclinada excêntrica inserida no domínio (Figura 6.39). Seja e=0,50 a distância entre o ponto médio da fissura e o eixo de simetria do domínio. Diferentemente dos problemas 6.4 e 6.5 , os fatores de intensidade de tensão são distintos para cada extremidade da fissura.

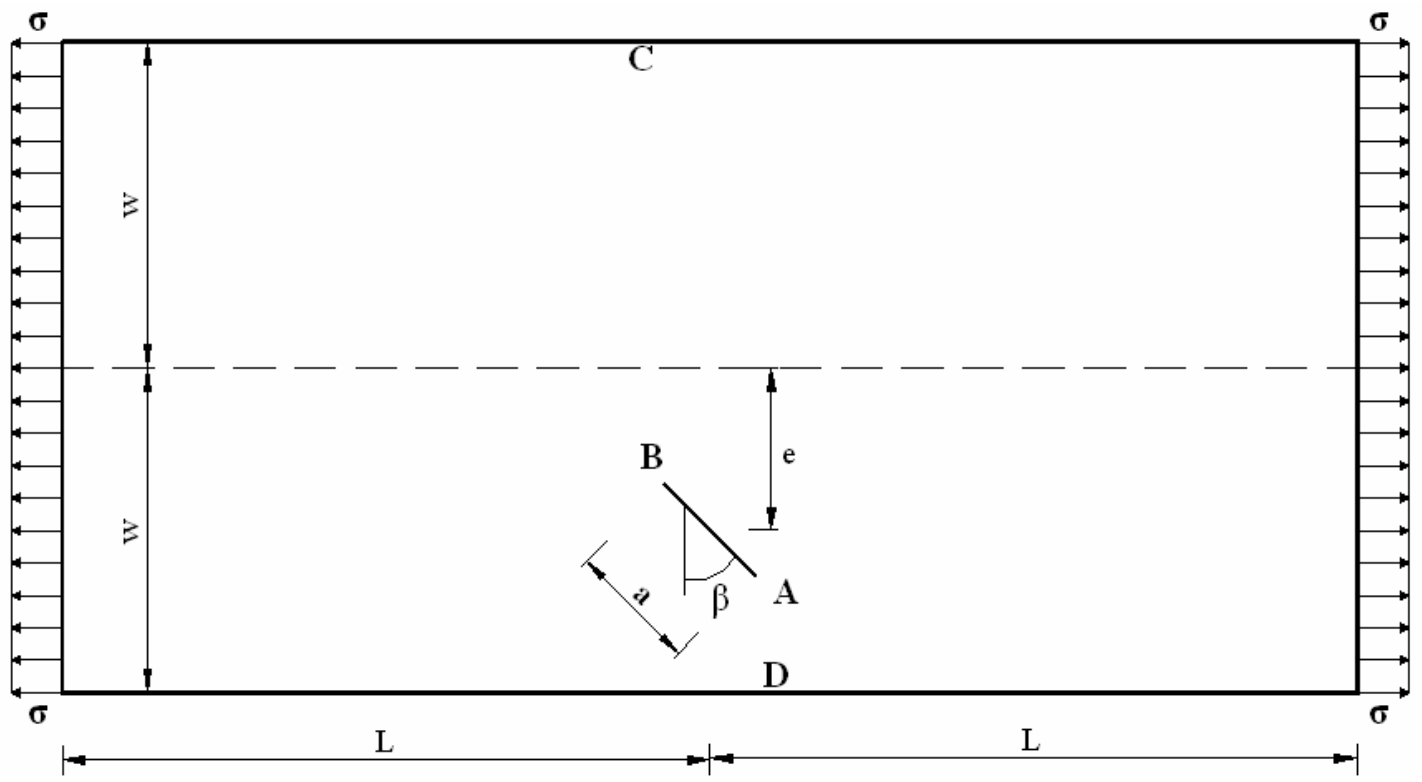

Figura 6.39 - Painel com fissura inclinada excêntrica e malha utilizada na análise.

O problema é idealizado com as seguintes condições:

- Estado Plano de Tensão (EPT);

- Coeficiente de Poisson $v=0,3$;

- $\quad$ Módulo de Young $\mathrm{E}=0$;

- Dimensões do domínio $\mathrm{w}=1,0, \mathrm{~L}=2,0$ e $\mathrm{a}=0,4$;

- Ângulo de inclinação da fissura $\beta=45^{\circ}$;

- Espessura unitária;

- Condições de contorno de Dirichlet: $\mathrm{u}_{\mathrm{xC}}=0,0, \mathrm{u}_{\mathrm{yC}}=0,0$ e $\mathrm{u}_{\mathrm{yD}}=0,0$;

- Condições de contorno de Neumman: $\sigma=1,0$. 
A Figura 6.40 apresenta a discretização utilizada no problema de fissura inclinada com excentricidade. Nessa análise, utilizou-se uma malha com 56 elementos quadrilaterais e uma taxa de progressão geométrica $q=0,15$ nas extremidade da fissura.

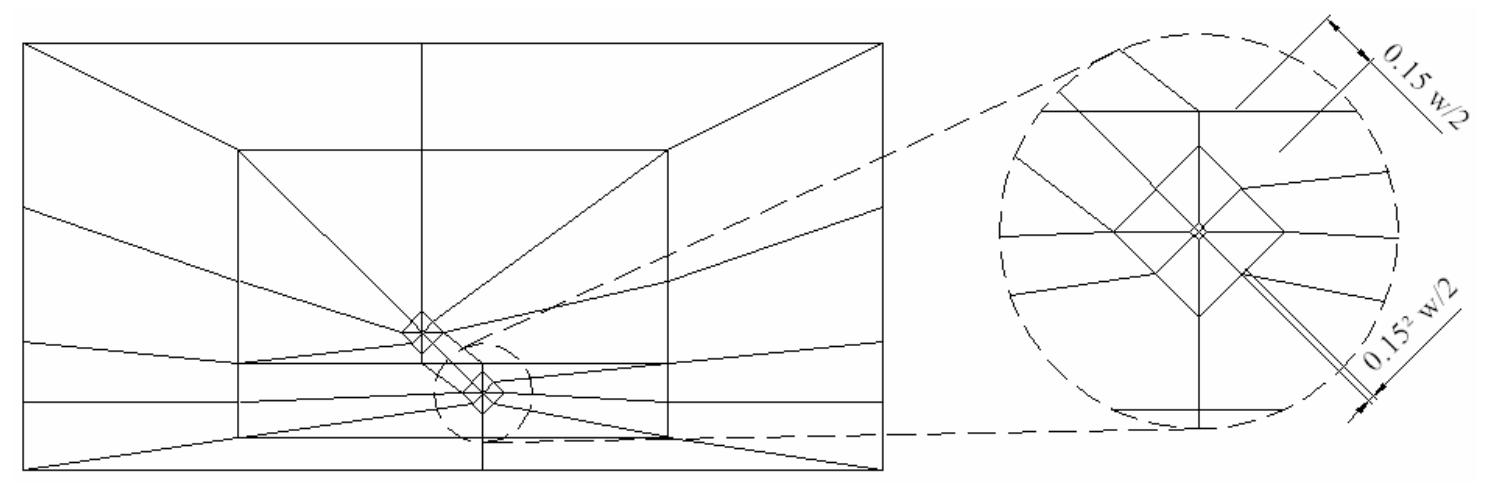

Figura 6.40 - Painel com fissura inclinada excêntrica e malha utilizada na análise.

Para a validação dos resultados da presente análise, foram utilizados os seguintes valores de referência para os fatores de intensidade de tensão normalizados:

para a extremidade A :

$$
\tilde{\mathrm{K}}_{\mathrm{IA}}=0,536 ; \quad \tilde{\mathrm{K}}_{\mathrm{IIA}}=0,525
$$

para a extremidade B :

$$
\tilde{\mathrm{K}}_{\mathrm{IB}}=0,538 ; \quad \tilde{\mathrm{K}}_{\mathrm{IIB}}=0,510
$$

esses valores podem ser encontrados em (MURAKAMI, 1978).

A relação entre os fatores de intensidade de tensão normalizados $(\tilde{K})$ e os fatores de intensidade de tensão $(\mathrm{K})$ pode ser escrita na forma:

$$
\tilde{\mathrm{K}}_{\mathrm{I}}=\frac{\mathrm{K}_{\mathrm{I}}}{\sigma \sqrt{\pi \mathrm{a} / 2}} ; \quad \tilde{\mathrm{K}}_{\mathrm{II}}=\frac{\mathrm{K}_{\mathrm{II}}}{\sigma \sqrt{\pi \mathrm{a} / 2}}
$$


A Figura 6.41 apresenta a magnitude do vetor deslocamento e a tensão de von Mises para a presente análise obtidas por meio do método dos elementos finitos generalizados com o enriquecimento polinomial $\mathrm{p}=7$.

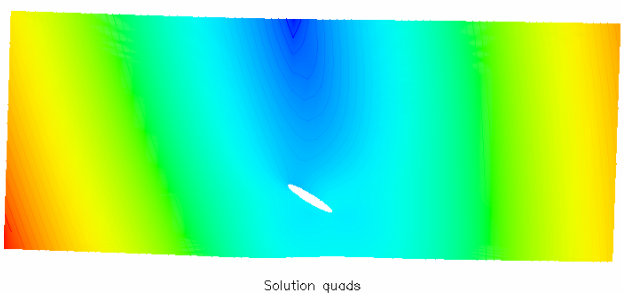

(a)
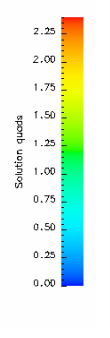

西

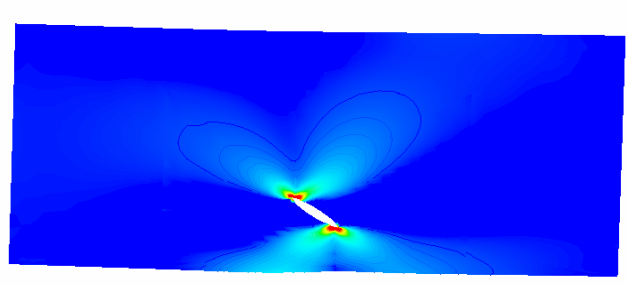

(b)

Figura 6.41 - (a) Magnitude do vetor deslocamento e (b) tensão de von Mises para o problema de fissura excêntrica

Utilizando os recursos da programação orientada a objetos, é possível criar objetos das classes de extração independentes para cada ponto de singularidade. Como os fatores de intensidade de tensão são diferentes para cada extremidade da fissura, foram utilizados dois objetos de extração. Cada objeto fornece os valores dos fatores de intensidade e da taxa de energia disponibilizada para cada extremidade de forma independente e sem a necessidade de modificação no código computacional. Na configuração dos domínios de integração, utilizou-se $\rho=0,02$ para o MIC e $\rho_{1}=0,02$ e $\rho_{2}=0,20$ para o MFC, para cada extremidade.

A Figura 6.42 e a Tabela 6.20 apresentam os fatores de intensidade de tensão para os modos I e II e o erro relativo comparando com os valores de referência para a extremidade A da fissura (6.21), respectivamente. 


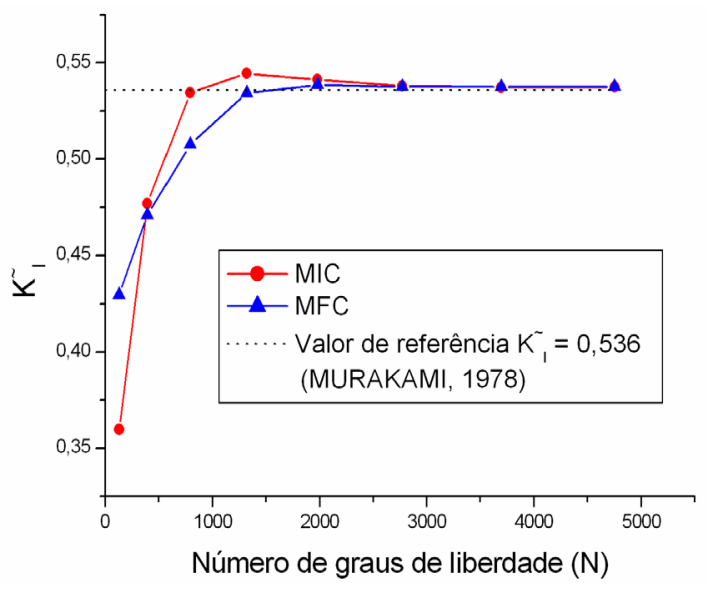

(a)

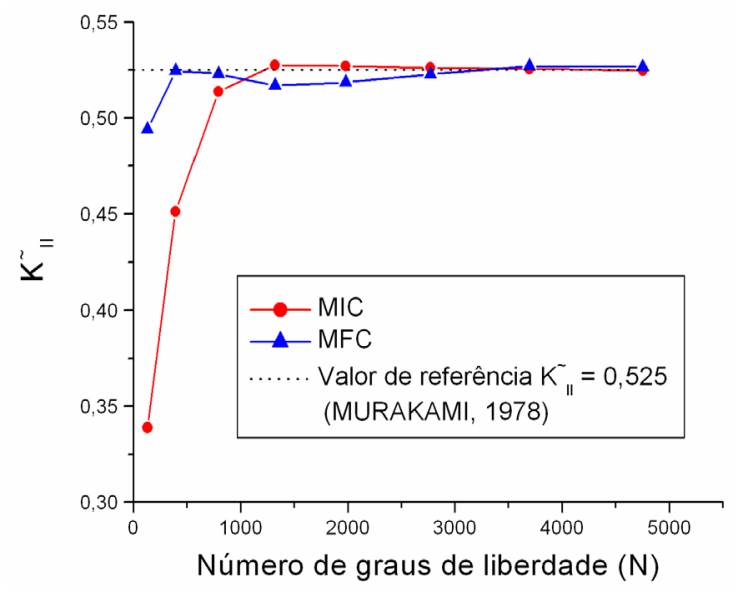

(b)

Figura 6.42 - Fatores de intensidade de tensão $K_{I}$ (a) e $K_{\text {II }}$ (b) para a extremidade A.

Tabela 6.20 - Erro relativo para os fatores de intensidade de tensão $K_{I}$ e $K_{\text {II }}$ na extremidade A, utilizando os métodos MIC e MFC.

\begin{tabular}{cccccc}
\hline $\mathrm{p}$ & $\mathrm{N}$ & $\begin{array}{c}\mathrm{er}\left(\mathrm{K}_{\mathrm{I}}\right) \% \\
\mathrm{MIC}\end{array}$ & $\begin{array}{c}\operatorname{er}\left(\mathrm{K}_{\mathrm{II}}\right) \% \\
\mathrm{MIC}\end{array}$ & $\begin{array}{c}\operatorname{er}\left(\mathrm{K}_{\mathrm{I}}\right) \% \\
\mathrm{MFC}\end{array}$ & $\begin{array}{c}\operatorname{er}\left(\mathrm{K}_{\mathrm{II}}\right) \% \\
\mathrm{MFC}\end{array}$ \\
\hline 1 & 132 & 32,885381 & 35,44505 & 19,80416 & 5,876824 \\
2 & 396 & 11,011328 & 14,05153 & 12,12391 & 0,079146 \\
3 & 792 & 0,268715 & 2,140185 & 5,307448 & 0,373752 \\
4 & 1320 & 1,595861 & 0,499012 & 0,323556 & 1,539439 \\
5 & 1980 & 1,004384 & 0,4089 & 0,509408 & 1,211672 \\
6 & 2772 & 0,384192 & 0,220025 & 0,292164 & 0,425176 \\
7 & 3696 & 0,253093 & 0,129673 & 0,286751 & 0,407218 \\
8 & 4752 & 0,268862 & 0,035172 & 0,295459 & 0,295479 \\
\hline
\end{tabular}

A Figura 6.43 ilustra a convergência dos fatores de intensidade de tensão $K_{I}$ e $\mathrm{K}_{\text {II }}$ para a extremidade $\mathrm{B}$ da fissura. A Tabela 6.21 apresenta os valores para o erro relativo dos fatores de intensidade de tensão, utilizando como base os valores de referência em (6.22). 


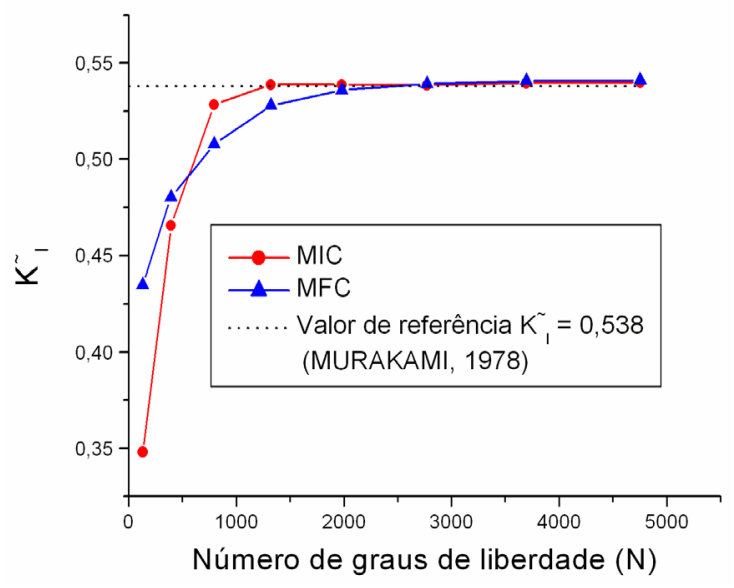

(a)

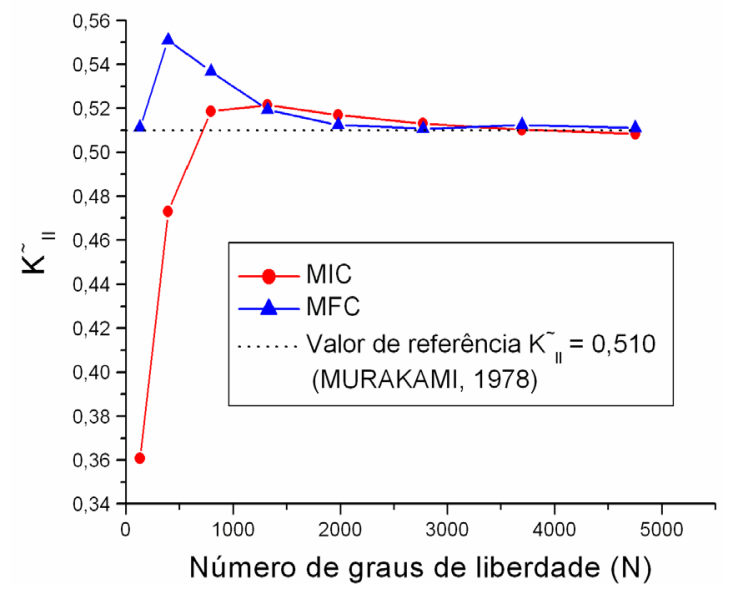

(b)

Figura 6.43 - Fatores de intensidade de tensão $\mathrm{K}_{\mathrm{I}}$ (a) e $\mathrm{K}_{\mathrm{II}}$ (b) para a extremidade $\mathrm{B}$.

Tabela 6.21 - Erro relativo para os fatores de intensidade de tensão $K_{I}$ e $K_{I I}$ na extremidade B, utilizando os métodos MIC e MFC.

\begin{tabular}{cccccc}
\hline $\mathrm{p}$ & $\mathrm{N}$ & $\begin{array}{c}\mathrm{er}\left(\mathrm{K}_{\mathrm{I}}\right) \% \\
\mathrm{MIC}\end{array}$ & $\begin{array}{c}\mathrm{er}\left(\mathrm{K}_{\mathrm{II}}\right) \% \\
\mathrm{MIC}\end{array}$ & $\begin{array}{c}\mathrm{er}\left(\mathrm{K}_{\mathrm{I}}\right) \% \\
\mathrm{MFC}\end{array}$ & $\begin{array}{c}\operatorname{er}\left(\mathrm{K}_{\mathrm{II}}\right) \% \\
\mathrm{MFC}\end{array}$ \\
\hline 1 & 132 & 35,29736 & 29,26621 & 19,15353 & 0,224266 \\
2 & 396 & 13,46617 & 7,266222 & 10,72941 & 8,038803 \\
3 & 792 & 1,784253 & 1,694114 & 5,62054 & 5,258657 \\
4 & 1320 & 0,144677 & 2,258851 & 1,850379 & 1,827939 \\
5 & 1980 & 0,152885 & 1,365612 & 0,352914 & 0,491421 \\
6 & 2772 & 0,100358 & 0,618567 & 0,21737 & 0,123588 \\
7 & 3696 & 0,308118 & 0,053089 & 0,521975 & 0,472374 \\
8 & 4752 & 0,386673 & 0,335276 & 0,590915 & 0,189882 \\
\hline
\end{tabular}

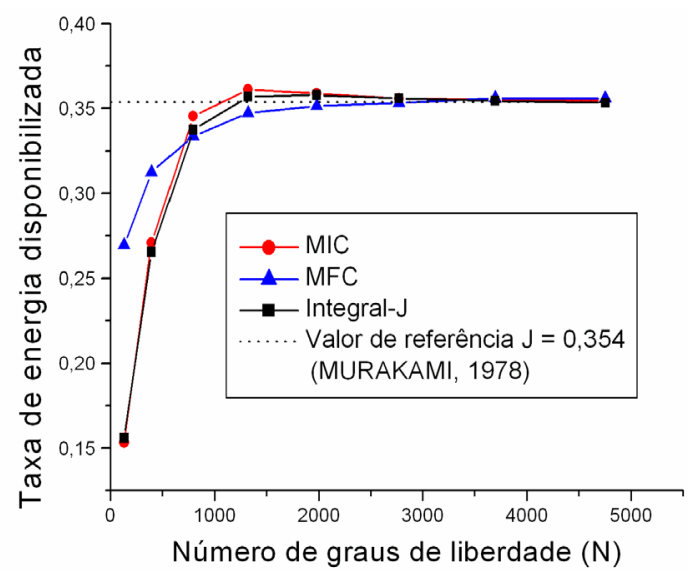

(a)

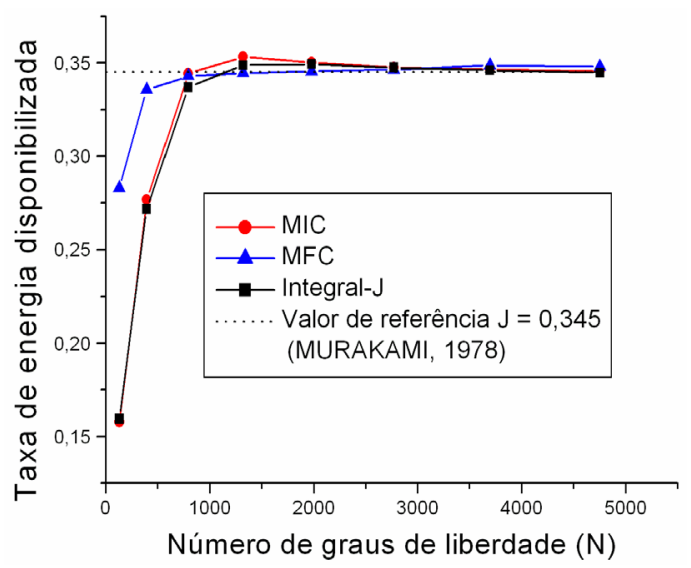

(b)

Figura 6.44 - Taxa de energia disponibilizada (a) para a extremidade A (b) para a extremidade B.

A Figura 6.44 apresenta convergência da taxa de energia disponibilizada para a propagação da fissura com o enriquecimento polinomial, utilizando os métodos MIC, 
MFC e Integral-J. O valor de referência para as taxas de energia disponibilizada em cada extremidade foi calculado utilizando os valores fornecidos em (6.21) e (6.22). Para a extremidade A :

$$
\mathscr{G}_{\mathrm{A}}=0,354
$$

para a extremidade B :

$$
\mathscr{G}_{\mathrm{B}}=0,345 \text {. }
$$

Nas análises dos fatores de intensidade de tensão e da taxa de energia disponibilizada para a propagação de fissuras, para as duas extremidades da fissura, os resultados apresentam-se de forma condizente com os valores encontrados na literatura.

O exemplo de fissura excêntrica, analisado com as implementações das classes de extração (Seção 5.3), ajuda a ilustrar a versatilidade de uma implementação computacional utilizando o paradigma da programação orientada a objetos. Com as implementações desenvolvidas no presente trabalho, é possível analisar, ao mesmo tempo, diversas fissuras inseridas completamente ou parcialmente no domínio. Para desenvolver análises com diversas fissuras, basta criar um objeto para cada ponto de singularidade presente no domínio. 


\section{Conclusão}

Nesta dissertação, diversos aspectos relacionados à extração de fatores de intensidade de tensão e da taxa de energia disponibilizada para a propagação de uma fissura, utilizando soluções numéricas, foram investigados.

Utilizou-se a solução do método dos elementos finitos generalizados (MEFG) nos experimentos numéricos. A solução numérica do MEFG foi obtida por meio do programa computacional Scientific and Engineering Toolkit (SET), desenvolvido pelo orientador do presente trabalho, que implementa a versão $p$ do MEFG. O programa SET foi implementado utilizando recursos da programação orientada a objetos, o que facilitou a reutilização do código computacional em diversas partes da implementação computacional dos métodos de extração.

A escolha do método da integral de contorno (MIC) e do método da função cutoff (MFC) para extração dos fatores de intensidade de tensão no contexto do MEFG é justificada pela generalidade e fundamentação matemática que suas formulações possuem e pela robustez e eficiência computacional inicialmente apresentada por Szabó e Babuška $(1988,1991)$ utilizando-se a solução do MEF na versão p. Em virtude da generalidade da formulação analítica dos métodos, dispensaram-se modificações na formulação do MIC e MFC quando aplicados ao MEFG. Algumas modificações aplicadas aos métodos MIC e MFC são relacionadas com aspectos de formulação numérica e implementação computacional.

A revisão e estudo dos métodos de extração de fatores de intensidade de tensão disponíveis para o MEF mostraram que uma das principais características apresentadas nesses métodos é que suas formulações numéricas são freqüentemente associadas à discretização utilizada na análise. A formulação numérica desenvolvida no presente 
trabalho apresenta-se de forma independente da discretização, facilitando assim uma futura análise de propagação de fissuras e mostrando-se bastante adequada na aplicação em método sem malha (PEREIRA; DUARTE, 2004).

Os valores extraídos com os métodos da integral de contorno (MIC) e da função cutoff (MFC) (SZABÓ, BABUŠKA, 1988) foram validados com vários resultados analíticos e numéricos encontrados na literatura. Na validação desses resultados, utilizou-se também o método da Integral-J (RICE, 1968), o qual é bastante conhecido e utilizado na literatura (MORAN; SHIH, 1987 a, b; NAHTA; MORAN, 1993; ORGAN, 1996; RAJU; SHIVAKUMAR, 1990).

Avaliou-se o desempenho dos métodos com a utilização de malhas geométricas na discretização dos domínios de análise e com o enriquecimento polinomial da solução do MEFG. Diversos problemas, variando a inclinação da fissura e a posição em relação ao eixo de simetria do domínio, foram utilizados para avaliar a robustez e a versatilidade da implementação computacional dos métodos.

\subsection{Considerações finais}

Com os desenvolvimentos apresentados, é possível discorrer algumas conclusões a respeito das técnicas de extração dos fatores de intensidade de tensão utilizadas no presente trabalho.

\subsubsection{Sobre as características dos métodos}

Alguns aspectos dos métodos de extração dos fatores de intensidade de tensão foram analisados.

Os resultados obtidos na análise de problemas clássicos da mecânica da fratura confirmam as hipóteses de superconvergência apresentadas por Babuška e Miller (1984a, b e c). Nas análises que comparam as taxas de convergência, observou-se que as taxas de convergência dos fatores de intensidade de tensão, extraído com o MIC e MFC, são maiores do que a taxa de convergência da solução do MEFG na norma energia.

Com relação à qualidade da solução, os erros relativos obtidos para os fatores de intensidade de tensão apresentam-se sempre entre o erro relativo na norma energia e o erro relativo na energia de deformação. 
A análise da variação do raio de integração na extração dos fatores de intensidade de tensão confirma a hipótese de independência do caminho de integração apresentada por Szabó e Babuška $(1988,1991)$.

\subsubsection{Sobre a formulação numérica}

Os métodos de extração implementados apresentaram-se de forma adequada no contexto do MEFG. As análises de independência do caminho de integração e da variação da inclinação e da posição da fissura no domínio exibem resultados que confirmam a robustez da implementação dos métodos de extração com relação à independência da discretização do problema e à generalidade da implementação computacional desenvolvida.

A formulação numérica utilizada na implementação dos métodos de extração apresenta-se bastante adequada aos métodos sem malha, visto que a extração dos fatores de intensidade de tensão é feita de forma independente da discretização utilizada na simulação. A característica de independência da discretização é particularmente importante em uma análise de propagação de fissuras.

Como esperado, os resultados obtidos com o método da função cutoff (MFC) apresentam-se de forma mais precisa e confiável do que nos métodos da integral de contorno (MIC) e na integral-J, o que justifica a maior complexidade de implementação do mesmo.

\subsubsection{Sobre a implementação computacional}

A análise de problemas com fissuras inclinadas confirmam a robustez da implementação utilizada para os métodos de extração. Tal robustez foi alcançada pelo desenvolvimento das transformações de coordenadas entre os espaços utilizados na integração numérica. As transformações utilizadas na implementação computacional dos métodos de extração permitiram a generalização do código, tornando-o aplicável a diversos problemas da mecânica da fratura.

A versatilidade encontrada na implementação, utilizando os recursos da programação orientada a objetos, facilita a análise de problemas mais gerais, como os de fissuras completamente inseridas no domínio, com ou sem excentricidade em relação aos eixos de simetria do domínio. Tal característica permite também que vários pontos 
de singularidade possam ser analisados ao mesmo tempo e de forma independente, sem qualquer alteração no código computacional.

A implementação computacional dos métodos foi desenvolvida de tal forma que sua extensão para o caso tridimensional pode ser feita da maneira mais simples possível. É possível desenvolver as extensões dos métodos para os casos tridimensionais criando subclasses na classe base de extração (ExtractSIF) (Seção 5.3).

\subsection{Propostas para trabalhos futuros}

Os métodos de extração abordados nesta dissertação possuem um campo de aplicação e de desenvolvimento que vai além do que foi abordado. Como forma de ampliar o desenvolvimento deste trabalho sugere-se as seguintes extensões:

- Aplicar os métodos da integral de contorno e da função cutoff utilizando outro tipo de método numérico que forneça a solução para problemas da elasticidade, por exemplo, o método dos elementos de contorno.

- Estudo da eficiência dos métodos de extração para problemas com diversas condições de contorno nas arestas. Ampliar as formulações apresentadas para a análise de problemas com tensões nas arestas da fissura.

- Desenvolvimento dos métodos de extração para problemas tridimensionais.

- Desenvolvimento de formulações dos métodos da função cutoff e da integral de contorno para problemas com materiais não homogêneos e problemas de fissuras em interface entre materiais.

- Aplicação dos métodos da função cutoff (MFC) e da integral de contorno (MIC) utilizando a implementação independente da discretização, em análise de propagação de fissuras.

- Utilização dos recursos da programação orientada a objetos e de programação paralela para analisar um domínio com diversas fissuras ao mesmo tempo. 


\section{Referências bibliográficas}

ALTAIR ENGINEERING (2004). Austin, Texas: Texas office of Altair Engineering. Disponível em: <http://www.comco.com>. Acesso em: 20 fev. 2004.

BABUŠKA, I.; MELENK, J. M. (1997). The partition of unity method. International journal for numerical methods in engineering, v.40, p.727-758.

BABUŠKA, I.; MILLER, A. (1984a). The post-processing approach in the finite element method - part 1: calculation of displacements, stresses and other higher derivates of the displacements. International journal for numerical methods in Engineering, v.20, p.1085-1109.

BABUŠKA, I.; MILLER, A. (1984b). The post-processing approach in the finite element method - part 2: the calculation of stress intensity factors. International journal for numerical methods in Engineering, v.20, p.1111-1129.

BABUŠKA, I.; MILLER, A. (1984c). The post-processing approach in the finite element method - part 3: a posteriori estimates and adaptive mesh selection. International journal for numerical methods in Engineering, v.20, p.2311-2324.

BARROS, F. B. (2002). Métodos sem malha e método dos elementos finitos generalizados em análise não-linear de estruturas. 172p. Tese (Doutorado) - Escola de Engenharia de São Carlos, Universidade de São Paulo, São Carlos. 2002.

BARTON, J. J.; NACKMAN, R. L. (1994). Scientific and Engineering C++: An Introduction with Advanced Techniques and Examples. New York: Addison-Wesley.

BRAVOS, C. M. A. A. (2004). Um sistema de refinamento H-P adaptativo utilizando elementos finitos hierárquicos multidimensionais. 246f. Tese (Doutorado) - Faculdade de Engenharia Mecânica, Universidade Estadual de Campinas, Campinas. 2004. 
BROEK, D. (1982). Elementary engineering fracture mechanics. 3rd ed. Hauge: Martinus Nijhof Publishers.

C++ BOOST (2004). Disponível em: <http://www.boost.org>. Acesso em: 8 mar. 2004.

CHANG, C.; MEAR, M. (1995). A boundary element method for two dimensional linear elastic fracture analysis. International journal of fracture, v.74, p.219-251.

CORNELL Fracture Group (2003). Ithaca, NY: Cornell University. Disponível em: $<\mathrm{http}$ ://www.cfg.cornell.edu/projects/spiral_bevel.html $>$.Acesso em: 10 mai. 2003.

DEITEL, H. M.; DEITEL, P. J. (2001). C++ how to program. New Jersey: Prentice Hall.

DEVLOO, Ph. R.; DUARTE, C. A.; NOEL A. T. (1992). On the Implementation of the p-Adaptive Finite Element Method Using the Object Oriented Programming Philosophy. In: INTERNATIONAL CONGRESS ON NUMERICAL METHODS IN ENGINEERING AND APPLIED SCIENCES, 1992, Concepcion, Chile, Anais... Concepcion.

DUARTE, C. A. M., Estudo da Versão p do Método de Elementos Finitos para Problemas da Elasticidade e de Potencial. 164p. Dissertação (Mestrado) Universidade Federal de Santa Catarina, Florianópolis. 1991.

DUARTE, C. A. M. (1996). The hp cloud method. 218p. Tese (Doutorado) University of Texas, Austin. 1996.

DUARTE, C. A.; BABUŠKA, I.; ODEN, J. T. (1999). Generalized finite element methods for three-dimensional structural mechanics problems. Computer and strucutres, v.77, p.215-232.

GOSZ, M.; DOLBOW, J.; MORAN, B. (1998). Domain integral formulation for stress intensity factor computation along curved three-dimensional interface cracks. International journal of solids structures, v.35, n.15, p.1763-1783.

HELLEN, T. K. (1975). On the method of virtual crack extension. International journal of numerical methods for engineering, v.9, p.187-207.

LI, F. Z.; SHIH, C. F.; NEEDLEMAN, A. (1985). A comparison of methods for calculating energy release rates. Engineering fracture mechanics, v.21, n.2, p.405421.

LIPPMAN, S. B.; LAJOIE, J. (1998). C++ Primer. New York: Addison Wesley. 
MORAN, B.; SHIH, C.F. (1987a). A general treatment of crack tip contour integrals. International journal of fracture, v.35, p.295-310.

MORAN, B.; SHIH, C.F. (1987b). Crack tip and associated domain integrals from momentum and energy balance. Engineering fracture mechanics, v.27, n.6, p.615642.

MURAKAMI, Y. (1978). Application of the body force method to the calculation of stress intensity factors for a crack in the arbitrarily shaped plate. Engineering fracture mechanics, v.10, p.497-513.

NAHTA, R.; MORAN, B. (1993). Domain integrals for axisymmetric crack problems. International journal of solids structures, v.30, n.15, p.2027-2040.

ODEN, J. T.; REDDY, J. N. (1976). An introduction to the mathematical theory of finite elements. New York: John Wiley and Sons.

ODEN, J. T.; DUARTE, C. A.; ZIENKIEWICZ, O. C. (1998). A new cloud-based hp finite element method. Computer methods in applied mechanics and engineering, v.153, p.117-126.

OPENDX (2004). Visualization Data Explorer. Missoula, MT: Visualization and Imagery Solutions. Disponível em: <http://www.opendx.org>. Acesso em: 8 fev. 2004.

ORGAN, D. J. (1996). Numerical solutions to dynamic fracture problems using the element-free Galerkin method. 159p. Tese (Doutorado) - Northwestern University, Evanston. 1996.

OWEN, D. R. J.; FAWKES A. J. (1983). Engineering fracture mechanics: numerical methods and application. Swansea: Pineridge Press..

PAPADAKIS, P.J; BABUŠKA, I. (1995). A numerical procedure for determination of certain quantities related to the stress intensity factors in two-dimensional elasticity. Computer methods in applied mechanics and engineering, v.122, p.69-92.

PARKS, D. M. (1974). A stiffness derivative finite element technique for determination of crack tip stress intensity factors. International journal of fracture, v.10, p.487-502.

PEREIRA, J. P. A.; DUARTE, C. A. M. (2003). Extraction of stress intensity factors from generalized finite element solutions. Engineering analysis with boundary elements. (submitted). 
RAJU, I. S.; SHIVAKUMAR, K. N. (1990). An equivalent domain integral method in the two-dimensional analysis of mixed mode crack problems. Engineering fracture mechanics, v.37, n.4, p.707-725.

RICE, J. R. (1968). A path independent integral and the approximate analysis of strain concentration by notches and cracks. Journal of applied mechanics, v.35, p.379-386.

STROBOULIS, T.; COPPS, K.; BABUŠKA I. (2000) The generalized finite element method; an example of its implementation and illustration of its performance. International journal for numerical methods in engineering, v.47, p.1401-1417.

SZABÓ, B. A. (1986). Mesh design for the p-version of the finite element method. Computer methods in applied mechanics and engineering, n.55, p.181-197.

SZABÓ, B.; BABUŠKA, I. (1988). Computation of the amplitude of stress singular terms for cracks and reentrant corners. Fracture mechanics: nineteenth symposium, ASTM STP 969, T. A. Cruse, Ed., Americam Society for Testing and Materials, Philadelphia, p.101-204.

SZABÓ, B.; BABUŠKA, I. (1991) Finite Element Analysis. New York: John Wiley and Sons.

TADA, H.; PARIS, P.; IRWIN G. (1973). Stress analysis of cracks handbook. St. Louis: Del Research Corporation.

VALLIAPAN, S. (1981). Continuum mechanics. Rotterdam: A. A. Balkema.

YAROSHENKO, O. (1994). The beginner's guide to C++. Birmingham: Wrox Press.

YOSIBASH, Z. (1997a). Computing edge singularities in elastic anisotropic threedimensional domains. International journal of fracture, v.86, p.221-245.

YOSIBASH, Z. (1997b) Finite element stress extraction by the complementary energy principle. International journal for numerical methods in engineering, v.40, p.13351354.

YOSIBASH, Z. (1997c). Numerical analysis of edge singularities in three-dimensional elasticity. International journal for numerical methods in engineering, v.40, p.46614632.

YOSIBASH, Z. (1997d). On solution of two-dimensional linear elastostatic and heattrasnfer problems in the vicinity of singular points. International journal of solids structures, v.34, n.2, p.243-274. 
YOSIBASH, Z. (1998). Thermal generalized stress intensity factors in 2-D domains. Computer methods in applied mechanics and engineering, v.157, p.365-385.

YOSIBASH, Z., SZABÓ, B. (1995a). The solution of axysimetric problems near singular points and computation of stress intensity factors. Finite elements analysis and design, v.19, p.115-129.

YOSIBASH, Z.; SZABÓ, B. (1995b). Generalized stress intensity factors in linear elastostatics. International journal of fracture, v.72, p.223-240.

YOSIBASH, Z.;SZABÓ, B. (1995c). Numerical analysis of singularities in twodimensions part 1: computation of eigenpairs. International journal for numerical methods in engineering, v.38, p.2055-2082.

YOSIBASH, Z.;SZABÓ, B. (1996). Numerical analysis of singularities in twodimensions part 2: computation of generalized flux/stress intensity factors. International journal for numerical methods in engineering, v.39, p.409-434. 


\section{Bibliografia consultada}

AKAY, T. J. (1994). Applied numerical methods for engineers. New York: John Wiley and Sons.

CARNAHAN, B.; LUTHER, H. A.; WILKES, J. O. (1969). Applied Numerical Methods. New York: John Wiley \& Sons.

HELLAN, K. (1984). Introduction to fracture mechanics. New York: McGraw-Hill Book Company.

HSU, H. P. (1969). Vector analysis. New York: Simon and Schuster.

KREYSIG, E. (1993). Advanced engineering mathematics. New York: John Wiley and Sons.

MEGUID, S. A. (1989). Engineering fracture mechanics. New York: Elsevier Applied Science.

STRESSCHECK, Release 6.1 (2001). Engineering software research and development, Inc.. Advanced Guide. 
APÊNDICE A - Transformação de coordenadas para o método da Integral-J 
Nesta seção, apresenta-se a transformação de coordenadas, entre os espaços local e global (Figura A.1), para as derivadas dos deslocamentos utilizadas na implementação do método da Integral-J.

Para a implementação do método da Integral-J utilizando a solução numérica do Método dos Elementos Finitos Generalizados (MEFG) em coordenadas globais, obtida com o programa SET (Capítulo 5), faz-se necessário o desenvolvimento de uma transformação de coordenadas que transforme as derivadas em coordenadas globais em derivadas nas coordenadas na ponta da fissura (Figura A.1).

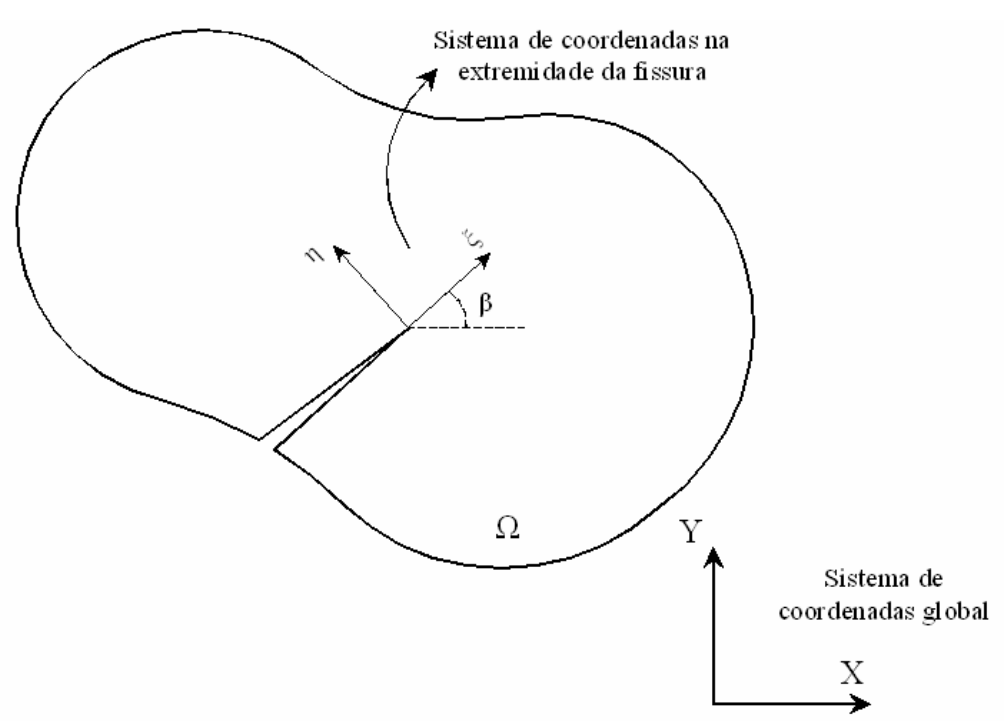

Figura A.1 - Sistemas de coordenadas local (extremidade da fissura) e global.

Definem-se as coordenadas dos espaços local e global e as respectivas representações dos campos de deslocamento nesses espaços.

- $\quad(\xi, \eta) \rightarrow$ coordenadas cartesianas do espaço local;

- $(\mathrm{x}, \mathrm{y}) \rightarrow$ coordenadas cartesianas do espaço global;

- $\quad\left(\hat{\mathrm{u}}_{\xi}(\xi, \eta), \hat{\mathrm{u}}_{\eta}(\xi, \eta)\right) \rightarrow$ campos de deslocamento no espaço local, em função das coordenadas cartesianas locais;

- $\left(\mathrm{u}_{\mathrm{x}}(\mathrm{x}, \mathrm{y}), \mathrm{u}_{\mathrm{y}}(\mathrm{x}, \mathrm{y})\right) \rightarrow$ campos de deslocamento no espaço global, em função das coordenadas cartesianas no espaço global;

A transformação que conduz as coordenadas do espaço local para o espaço global pode ser escrita da seguinte forma: 


$$
\begin{aligned}
& \mathrm{T}_{2}:(\xi, \eta) \rightarrow(\mathrm{x}, \mathrm{y}) \\
& \left\{\begin{array}{l}
\mathrm{x} \\
\mathrm{y}
\end{array}\right\}=\left\{\begin{array}{l}
\mathrm{x}_{\mathrm{o}} \\
\mathrm{y}_{\mathrm{o}}
\end{array}\right\}+\left[\begin{array}{cc}
\cos (\beta) & -\operatorname{sen}(\beta) \\
\operatorname{sen}(\beta) & \cos (\beta)
\end{array}\right]\left\{\begin{array}{l}
\xi \\
\eta
\end{array}\right\},
\end{aligned}
$$

a matriz da transformação é:

$$
[R]=\left[\begin{array}{ll}
\frac{\partial x}{\partial \xi} & \frac{\partial x}{\partial \eta} \\
\frac{\partial y}{\partial \xi} & \frac{\partial y}{\partial \eta}
\end{array}\right]=\left[\begin{array}{cc}
\cos (\beta) & -\operatorname{sen}(\beta) \\
\operatorname{sen}(\beta) & \cos (\beta)
\end{array}\right]
$$

Para os deslocamentos tem-se:

$$
\begin{aligned}
& \mathrm{T}_{3}:\left(\mathrm{u}_{\mathrm{x}}, \mathrm{u}_{\mathrm{y}}\right) \rightarrow\left(\hat{\mathrm{u}}_{\xi}, \hat{\mathrm{u}}_{\mathrm{\eta}}\right) \\
& \left\{\begin{array}{c}
\hat{\mathrm{u}}_{\xi} \\
\hat{\mathrm{u}}_{\eta}
\end{array}\right\}=\left[\begin{array}{cc}
\cos (\beta) & \operatorname{sen}(\beta) \\
-\operatorname{sen}(\beta) & \cos (\beta)
\end{array}\right]\left\{\begin{array}{l}
\mathrm{u}_{\mathrm{x}} \\
\mathrm{u}_{\mathrm{y}}
\end{array}\right\}=\mathrm{R}^{\mathrm{T}}\left\{\begin{array}{l}
\mathrm{u}_{\mathrm{x}} \\
\mathrm{u}_{\mathrm{y}}
\end{array}\right\}
\end{aligned}
$$

De (A.3) e como (A.2) é constante, usando a regra da cadeia, tem-se:

$$
\begin{gathered}
\left\{\begin{array}{c}
\frac{\partial \hat{\mathrm{u}}_{\xi}}{\partial \xi} \\
\frac{\partial \hat{\mathrm{u}}_{\eta}}{\partial \xi}
\end{array}\right\}=[\mathrm{R}]^{\mathrm{T}}\left[\begin{array}{cc}
\frac{\partial \mathrm{u}_{\mathrm{x}}}{\partial \mathrm{x}} & \frac{\partial \mathrm{u}_{\mathrm{x}}}{\partial \mathrm{y}} \\
\frac{\partial \mathrm{u}_{\mathrm{y}}}{\partial \mathrm{x}} & \frac{\partial \mathrm{u}_{\mathrm{y}}}{\partial \mathrm{y}}
\end{array}\right]\left\{\begin{array}{c}
\frac{\partial \mathrm{x}}{\partial \xi} \\
\frac{\partial \mathrm{y}}{\partial \xi}
\end{array}\right\}, \\
\left\{\begin{array}{c}
\frac{\partial \hat{\mathrm{u}}_{\xi}}{\partial \eta} \\
\frac{\partial \hat{\mathrm{u}}_{\eta}}{\partial \eta}
\end{array}\right\}=[\mathrm{R}]^{\mathrm{T}}\left[\begin{array}{cc}
\frac{\partial \mathrm{u}_{\mathrm{x}}}{\partial \mathrm{x}} & \frac{\partial \mathrm{u}_{\mathrm{x}}}{\partial \mathrm{y}} \\
\frac{\partial \mathrm{u}_{\mathrm{y}}}{\partial \mathrm{x}} & \frac{\partial \mathrm{u}_{\mathrm{y}}}{\partial \mathrm{y}}
\end{array}\right]\left\{\begin{array}{l}
\frac{\partial \mathrm{x}}{\partial \eta} \\
\frac{\partial \mathrm{y}}{\partial \eta}
\end{array}\right\} .
\end{gathered}
$$

A relação entre as derivadas do campo de deslocamentos em coordenadas globais, com relação às coordenadas globais, e as derivadas do campo de deslocamentos em coordenadas locais, com relação às coordenadas locais, pode ser escrita na forma:

$$
\left[\mathrm{D} \hat{u}_{\xi \eta}\right]=[\mathrm{R}]^{\mathrm{T}} \cdot\left[\mathrm{Du}_{\mathrm{xy}}\right] \cdot[\mathrm{R}]
$$


sendo:

$$
\begin{gathered}
{\left[D \hat{u}_{\xi \eta}\right]=\left[\begin{array}{ll}
\frac{\partial \hat{u}_{\xi}}{\partial \xi} & \frac{\partial \hat{u}_{\xi}}{\partial \eta} \\
\frac{\partial \hat{u}_{\eta}}{\partial \xi} & \frac{\partial \hat{u}_{\eta}}{\partial \eta}
\end{array}\right],} \\
{\left[D u_{x y}\right]=\left[\begin{array}{ll}
\frac{\partial u_{x}}{\partial x} & \frac{\partial u_{x}}{\partial y} \\
\frac{\partial u_{y}}{\partial x} & \frac{\partial u_{y}}{\partial y}
\end{array}\right] .}
\end{gathered}
$$

Com as transformações apresentadas nesta seção, é possível obter as derivadas dos deslocamentos em coordenadas locais com relação às coordenadas locais (Equação (A.7)) a partir das derivadas dos deslocamentos em coordenadas globais com relação às coordenadas globais (Equação (A.8)). 
APÊNDICE B - Desenvolvimento analítico para os coeficientes da função extratora 
Nesta seção, apresenta-se o cálculo das integrais que fornecem os coeficientes das funções extratoras e a verificação da propriedade de ortogonalidade das autofunções apresentados na Seção 3.2.

No desenvolvimento das integrais para a determinação dos coeficientes da função extratora utilizou-se o aplicativo MAPLE 7. Com o intuito de documentar e facilitar uma futura reutilização do desenvolvimento apresentado nesta seção, segue o desenvolvimento da integração analítica na forma do código utilizado no aplicativo MAPLE 7.

\section{Pacotes do $M A P L E 7$ utilizados no desenvolvimento dos coeficientes da função extratora}

$>$ restart:

$>$ with (linalg):

\section{Definições das funções $\psi$ e $\Upsilon$}

Para o modo I (considerando autovalores positivos)

$>$ Lambda1:=(i) $->($ lambda1 [i] -1)/(1ambda1 [i] +1) ;

$$
\Lambda 1:=i \rightarrow \frac{\lambda 1_{i}-1}{\lambda 1_{i}+1}
$$

$>Q 1:=$ (i) ->- Lambda1 (i)*sin((lambda1[i]1)*alpha/2)/sin ( (lambda1 [i]+1)*alpha/2);

$$
Q 1:=i \rightarrow-\frac{\Lambda 1(i) \sin \left(\frac{1}{2}\left(\lambda 1_{i}-1\right) \alpha\right)}{\sin \left(\frac{1}{2}\left(\lambda 1_{i}+1\right) \alpha\right)}
$$

Autofunções para Modo I:

$>$ psil := (theta, $i)->$ matrix([ [ (kappa Q1 (i) * (lambda1 [i] +1)) *cos (lambda1 [i] *theta) lambda1 [i]*cos ( (lambda1 [i] -2$) *$ theta) ], [ (kappa + Q1 (i) * (lambda1 [i] +1))*sin (lambda1 [i] *theta) +lambda1 [i] *sin( (lambda1 [i] -2$) *$ theta) ] ]) ;

$$
\psi 1:=(\theta, i) \rightarrow\left[\begin{array}{c}
\left(\kappa-\mathrm{Q} 1(i)\left(\lambda 1_{i}+1\right)\right) \cos \left(\lambda 1_{i} \theta\right)-\lambda 1_{i} \cos \left(\left(\lambda 1_{i}-2\right) \theta\right) \\
\left(\kappa+\mathrm{Q} 1(i)\left(\lambda 1_{i}+1\right)\right) \sin \left(\lambda 1_{i} \theta\right)+\lambda 1_{i} \sin \left(\left(\lambda 1_{i}-2\right) \theta\right)
\end{array}\right]
$$

Tensões para Modo I (apenas os fatores que são dependentes de $\theta$ ):

$>\operatorname{sigma11}:=$ (theta, $i)->(2-$

$Q 1(i) *(1 \operatorname{ambda} 1[i]+1)) * \cos (($ lambda1 [i] -1) *theta) -

(lambda1 [i] -1)* $\cos$ ( (lambda1 [i]-3)*theta) ;

$\sigma 11:=(\theta, i) \rightarrow\left(2-\mathrm{Q} 1(i)\left(\lambda 1_{i}+1\right)\right) \cos \left(\left(\lambda 1_{i}-1\right) \theta\right)-\left(\lambda 1_{i}-1\right) \cos \left(\left(\lambda 1_{i}-3\right) \theta\right)$ 


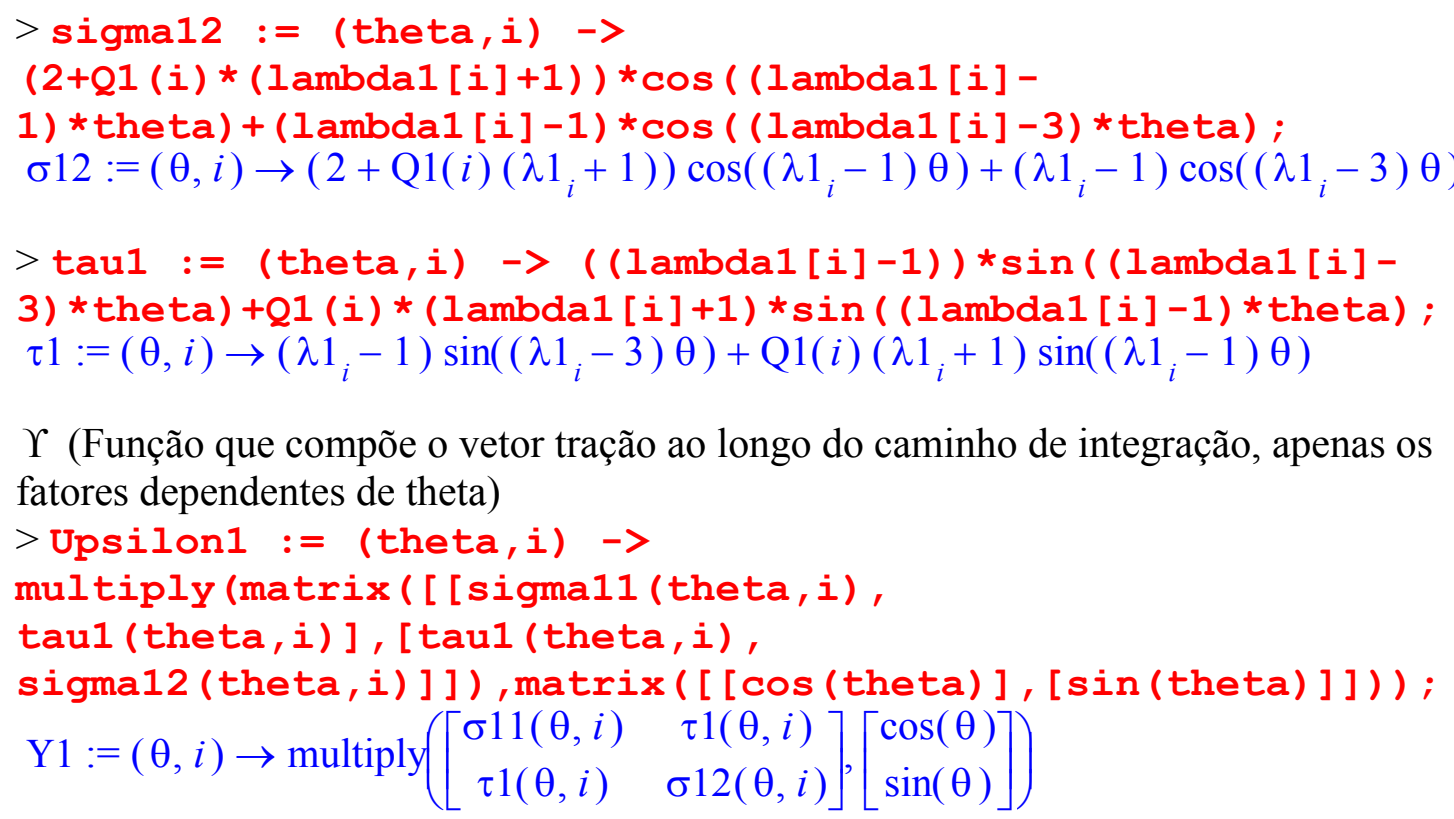

\section{Para o modo I (considerando autovalores negativos)}

> Lambdaj1:=(i) -> (lambdaj1 [i]-1)/(lambdaj1 [i] +1) ;

$$
\text { Lambdaj1 }:=i \rightarrow \frac{\text { lambdaj }_{i}-1}{\text { lambdaj }_{i}+1}
$$

$>Q j 1:=$ (i) $->-$ Lambdaj1(i)*sin((lambdaj1[i]1) *alpha/2)/sin ( (lambdaj1[i]+1)*alpha/2);

$$
Q j 1:=i \rightarrow-\frac{\text { Lambdaj1 }(i) \sin \left(\frac{1}{2}\left(\operatorname{lambdaj}_{i}-1\right) \alpha\right)}{\sin \left(\frac{1}{2}\left(\operatorname{lambdaj}_{i}+1\right) \alpha\right)}
$$

Autofunções para Modo I (autovalores negativos):

$>$ psij1 := (theta,i) $->$ matrix([[ (kappa Qj1 (i) * (lambdaj1[i]+1))*cos (lambdaj1 [i] *theta) lambdaj1[i]*cos ( (lambdaj1[i]-2)*theta)], [ (kappa + $2 j 1$ (i) * (lambdaj1 [i] +1) ) * sin (lambdaj1 [i] *theta) +lambdaj1 [i] * $\sin ((\operatorname{lambdaj} 1[i]-2) *$ theta) ] ]);

$p \operatorname{pij} 1:=(\theta, i) \rightarrow\left[\begin{array}{c}\left(\kappa-\mathrm{Qj} 1(i)\left(\operatorname{lambdaj}_{i}+1\right)\right) \cos \left(\operatorname{lambdaj}_{i} \theta\right)-\operatorname{lambdaj}_{i} \cos \left(\left(\operatorname{lambdaj}_{i}-2\right) \theta\right) \\ \left(\kappa+\mathrm{Qj} 1(i)\left(\operatorname{lambdaj}_{i}+1\right)\right) \sin \left(\operatorname{lambdaj}_{i} \theta\right)+\operatorname{lambdaj}_{i} \sin \left(\left(\operatorname{lambdaj}_{i}-2\right) \theta\right)\end{array}\right]$

Tensões para Modo I (autovalores negativos, apenas os fatores que são dependentes de $\theta)$ :

$>\operatorname{sigmaj11}:=$ (theta, i) $\rightarrow$ (2-

Qj1 (i) * (lambdaj1 [i]+1))* $\cos ((\operatorname{lambdaj1[i]-1)*theta)~-~}$

(lambdaj1 [i]-1)* cos ( (lambdaj1[i]-3)*theta);

$\operatorname{sigmaj} 11:=(\theta, i) \rightarrow\left(2-\operatorname{Qj} 1(i)\left(\operatorname{lambdaj}_{i}+1\right)\right) \cos \left(\left(\operatorname{lambdaj}_{i}-1\right) \theta\right)$

$-\left(\operatorname{lambdaj}_{i}-1\right) \cos \left(\left(\operatorname{lambdaj}_{i}-3\right) \theta\right)$ 
$>\operatorname{sigmaj} 12:=($ theta, $i)->$

$(2+Q j 1$ (i) * (lambdaj1[i] +1$)) * \cos ($ (lambdaj1 [i] -

$1)$ *theta $)+($ lambdaj1 [i]-1)* $\cos (($ lambdaj1 [i]-3) *theta) ;

$\operatorname{sigmaj} 12:=(\theta, i) \rightarrow\left(2+\operatorname{Qj} 1(i)\left(\operatorname{lambdaj}_{i}+1\right)\right) \cos \left(\left(\operatorname{lambdaj}_{i}-1\right) \theta\right)$

$+\left(\operatorname{lambdaj}_{i}-1\right) \cos \left(\left(\operatorname{lambdaj}_{i}-3\right) \theta\right)$

$>\operatorname{tauj1}:=($ theta,$i)->(($ lambdaj1[i]-1))*sin((lambdaj1[i]-

3) *theta) $+Q j 1$ (i) * (lambdaj1 [i] +1) * sin ( (lambdaj1 [i] -

1) *theta) ;

tauj $1:=(\theta, i) \rightarrow\left(\right.$ lambdaj $\left._{i}-1\right) \sin \left(\left(\right.\right.$ lambdaj $\left.\left._{i}-3\right) \theta\right)$

+ Qj1 $(i)\left(\operatorname{lambdaj}_{i}+1\right) \sin \left(\left(\operatorname{lambdaj}_{i}-1\right) \theta\right)$

$\Upsilon$ (Função que compõe o vetor tração ao longo do caminho de integração, apenas os fatores dependentes de theta)

$>$ Upsilonj1 := (theta, i) $->$

multiply (matrix ([ [sigmaj11 (theta, i),

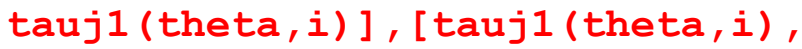

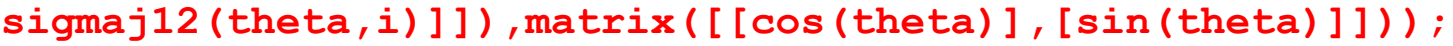

Upsilonj1 $:=(\theta, i) \rightarrow$ multiply $\left(\left[\begin{array}{cc}\operatorname{sigmaj} 11(\theta, i) & \operatorname{tauj} 1(\theta, i) \\ \operatorname{tauj} 1(\theta, i) & \operatorname{sigmaj} 12(\theta, i)\end{array}\right],\left[\begin{array}{c}\cos (\theta) \\ \sin (\theta)\end{array}\right]\right)$

\section{Para o modo II (considerando autovalores positivos)}

$>Q 2:=$ (i) $->-\sin ($ (lambda2[i] -

1)*alpha/2)/sin ( (lambda2[i]+1)*alpha/2);

$$
Q 2:=i \rightarrow-\frac{\sin \left(\frac{1}{2}\left(\lambda 2_{i}-1\right) \alpha\right)}{\sin \left(\frac{1}{2}\left(\lambda 2_{i}+1\right) \alpha\right)}
$$

Autofunções para Modo II:

$>$ psi2 := (theta, i) -> matrix([[ (kappa -

Q2 (i) * (lambda2 [i] +1)) *sin (lambda2 [i] *theta) -

lambda2 [i]*sin ( (lambda2 [i]-2)*theta) ], [-( (kappa +

Q2 (i) * (lambda2 [i] +1)) * cos (lambda2 [i] *theta) +lambda2 [i] *cos ( (lambda2 [i]-2)*theta) ) ] ]);

$\psi 2:=(\theta, i) \rightarrow\left[\begin{array}{c}\left(\kappa-\mathrm{Q} 2(i)\left(\lambda 2_{i}+1\right)\right) \sin \left(\lambda 2_{i} \theta\right)-\lambda 2_{i} \sin \left(\left(\lambda 2_{i}-2\right) \theta\right) \\ -\left(\kappa+\mathrm{Q} 2(i)\left(\lambda 2_{i}+1\right)\right) \cos \left(\lambda 2_{i} \theta\right)-\lambda 2_{i} \cos \left(\left(\lambda 2_{i}-2\right) \theta\right)\end{array}\right]$

Tensões para Modo II (apenas os fatores que são dependentes de $\theta$ ):

$>$ sigma21 := (theta, i) $\rightarrow$ (2-

Q2 (i) * (lambda2 [i] +1)) * sin ( (lambda2 [i] -1) *theta) -

(lambda2 [i]-1)*sin ( lambda2 [i]-3)*theta);

$\sigma 21:=(\theta, i) \rightarrow\left(2-\mathrm{Q} 2(i)\left(\lambda 2_{i}+1\right)\right) \sin \left(\left(\lambda 2_{i}-1\right) \theta\right)-\left(\lambda 2_{i}-1\right) \sin \left(\left(\lambda 2_{i}-3\right) \theta\right)$

$>$ sigma22 $:=($ theta, $i)->$

$(2+Q 2$ (i) * (lambda2 [i] +1)) * sin ( lambda2 [i] -

1) *theta) + (lambda2 [i] -1)* $\sin ($ (lambda2 [i] -3) *theta);

$\sigma 22:=(\theta, i) \rightarrow\left(2+\mathrm{Q} 2(i)\left(\lambda 2_{i}+1\right)\right) \sin \left(\left(\lambda 2_{i}-1\right) \theta\right)+\left(\lambda 2_{i}-1\right) \sin \left(\left(\lambda 2_{i}-3\right) \theta\right)$ 
$>$ tau2 := (theta, $i)->-((($ lambda2 [i] -1$)) * \cos (($ lambda2 [i] $3)$ *theta $)+Q 2(i) *(1$ ambda $2[i]+1) * \cos (($ lambda2 $[i]-1)$ *theta $))$; $\tau 2:=(\theta, i) \rightarrow-\left(\lambda 2_{i}-1\right) \cos \left(\left(\lambda 2_{i}-3\right) \theta\right)-\mathrm{Q} 2(i)\left(\lambda 2_{i}+1\right) \cos \left(\left(\lambda 2_{i}-1\right) \theta\right)$

$\Upsilon$ (Função que compõe o vetor tração ao longo do caminho de integração, apenas os fatores dependentes de $\theta$ )

$>$ Upsilon2 := (theta, i) -> multiply (matrix ([ [sigma21 (theta, i), tau2 (theta, i) ], [tau2 (theta, i), sigma22(theta, i) ] ]), matrix ([ [ cos (theta) ], [sin(theta) ] ])); $\mathrm{Y} 2:=(\theta, i) \rightarrow$ multiply $\left(\left[\begin{array}{cc}\sigma 21(\theta, i) & \tau 2(\theta, i) \\ \tau 2(\theta, i) & \sigma 22(\theta, i)\end{array}\right],\left[\begin{array}{c}\cos (\theta) \\ \sin (\theta)\end{array}\right]\right)$

\section{Para o modo II (considerando autovalores negativos)}

$>Q j 2:=$ (i) $->-\sin ($ (lambdaj2[i] -

1)*alpha/2)/sin ( (lambdaj2[i]+1)*alpha/2);

$$
Q j 2:=i \rightarrow-\frac{\sin \left(\frac{1}{2}\left(\operatorname{lambdaj}_{i}-1\right) \alpha\right)}{\sin \left(\frac{1}{2}\left(\operatorname{lambdaj}_{i}+1\right) \alpha\right)}
$$

Autofunções para modo II (considerando autovalores negativos):

$>$ psij2 := (theta,i) $\rightarrow$ matrix([ [ kappa -

Qj2 (i) * (lambdaj2[i]+1)) * sin (lambdaj2 [i] * theta) -

lambdaj2[i]*sin ( (lambdaj2[i]-2)*theta)], [-( (kappa +

Qj2 (i) * (lambdaj2 [i] +1)) * cos (lambdaj2 [i] * theta) +lambdaj2 [i] * $\cos (($ lambdaj2 [i]-2)*theta)) ] ]);

$p \operatorname{sij} 2:=(\theta, i) \rightarrow\left[\begin{array}{c}\left(\kappa-\operatorname{Qj} 2(i)\left(\operatorname{lambdaj}_{i}+1\right)\right) \sin \left(\operatorname{lambdaj}_{i} \theta\right)-\operatorname{lambdaj}_{i} \sin \left(\left(\operatorname{lambdaj}_{i}-2\right) \theta\right) \\ -\left(\kappa+\operatorname{Qj} 2(i)\left(\operatorname{lambdaj}_{i}+1\right)\right) \cos \left(\operatorname{lambdaj}_{i} \theta\right)-\operatorname{lambdaj} 2_{i} \cos \left(\left(\operatorname{lambdaj}_{i}-2\right) \theta\right)\end{array}\right]$

Tensões para modo II (considerando autovalores negativos, apenas os fatores que são dependentes de $\theta)$ :

$>$ sigmaj21:= (theta, $i)->(2-$

Qj2 (i) * (lambdaj2[i]+1)) *sin ( (lambdaj2 [i] -1) *theta) -

(lambdaj2 [i]-1)*sin ( (lambdaj2[i]-3)*theta) ;

$\operatorname{sigmaj} 21:=(\theta, i) \rightarrow\left(2-\operatorname{Qj} 2(i)\left(\right.\right.$ lambdaj $\left.\left._{i}+1\right)\right) \sin \left(\left(\right.\right.$ lambdaj $\left.\left._{i}-1\right) \theta\right)$

$-\left(\right.$ lambdaj $\left._{i}-1\right) \sin \left(\left(\right.\right.$ lambdaj $\left.\left._{i}-3\right) \theta\right)$

$>$ sigmaj22 := (theta, $i)->$

$(2+Q j 2(i) *($ lambdaj2[i] +1) )* sin ( (lambdaj2 [i] -

$1)$ *theta $)+($ lambdaj2 [i] -1)*sin ( lambdaj2 [i]-3) *theta) ;

$\operatorname{sigmaj} 22:=(\theta, i) \rightarrow\left(2+\operatorname{Qj} 2(i)\left(\right.\right.$ lambdaj $\left.\left._{i}+1\right)\right) \sin \left(\left(\right.\right.$ lambdaj $\left.\left._{i}-1\right) \theta\right)$

$+\left(\right.$ lambdaj $\left._{i}-1\right) \sin \left(\left(\right.\right.$ lambdaj $\left.\left._{i}-3\right) \theta\right)$

$>$ tauj2 := (theta, $i)->-(($ (lambdaj2[i]-

$1)) * \cos ($ (lambdaj2 [i] -

$3) *$ theta) $+Q j 2$ (i) * (lambdaj2[i] +1)* $\cos ($ (lambdaj2 [i] -

1)*theta)) ; 


$$
\begin{gathered}
\operatorname{tauj} 2:=(\theta, i) \rightarrow-\left(\operatorname{lambdaj}_{i}-1\right) \cos \left(\left(\operatorname{lambdaj}_{i}-3\right) \theta\right) \\
-\mathrm{Qj} 2(i)\left(\operatorname{lambdaj}_{i}+1\right) \cos \left(\left(\operatorname{lambdaj}_{i}-1\right) \theta\right)
\end{gathered}
$$

$\Upsilon$ (Função que compõe o vetor tração ao longo do caminho de integração, apenas os fatores dependentes de $\theta$ )

$>$ Upsilonj2 := (theta, i) $->$

multiply (matrix ([ [sigmaj21 (theta, i), tauj2(theta,i)], [tauj2(theta,i), sigmaj22(theta,i) ] ]), $\operatorname{matrix}([[\cos ($ theta) ], [sin(theta) ] ])) ; Upsilonj $:=(\theta, i) \rightarrow$ multiply $\left(\left[\begin{array}{cc}\operatorname{sigmaj} 21(\theta, i) & \operatorname{tauj} 2(\theta, i) \\ \operatorname{tauj} 2(\theta, i) & \operatorname{sigmaj} 22(\theta, i)\end{array}\right],\left[\begin{array}{c}\cos (\theta) \\ \sin (\theta)\end{array}\right]\right)$

\section{Definição da integral $C_{(i, j)}^{(m, n)}$}

\section{Para m diferente de $n$}

$>$ It : $=($ theta $, i, j)-$

>lambda1 [i] *multiply (transpose (psij2 (theta, j)),

(Upsilon1 (theta,i))) $[1,1]+$ lambda2[j] * multiply (

transpose (psi1 (theta, i) ), (Upsilonj2(theta, j) )) [1,1];

It $:=(\theta, i, j) \rightarrow \lambda 1_{i}$ multiply $(\operatorname{transpose}(\operatorname{psij} 2(\theta, j)), \mathrm{Y} 1(\theta, i))_{1,1}$

$+\lambda 2_{j}$ multiply( $\left.\operatorname{transpose}(\psi 1(\theta, i)), \operatorname{Upsilonj} 2(\theta, j)\right)_{1,1}$

$>C:=(i, j)->(1 /(2 * G))$ *int (It (theta, $i, j)$, theta=-

alpha/2..alpha/2);

$$
C:=(i, j) \rightarrow \frac{1}{2} \frac{\int_{-1 / 2 \alpha}^{1 / 2 \alpha} \operatorname{It}(\theta, i, j) d \theta}{G}
$$

\section{Para $\mathbf{m}=\mathbf{n}=\mathbf{1}$}

$>$ C11 := (i,j) $->(1 /(2 * G)) *$ int (

lambda1 [i] *multiply (transpose (psij1 (theta,j)),

(Upsilon1 (theta, i)) ) $[1,1]+$ lambdal [j] * multiply (

transpose (psi1 (theta, i) ), Upsilonj1(theta, j)) $[1,1]$, theta=alpha/2..alpha/2);

$C 11:=(i, j) \rightarrow \frac{1}{2} \int_{-1 / 2 \alpha}^{1 / 2 \alpha} \lambda 1_{i} \operatorname{multiply}(\operatorname{transpose}(\operatorname{psij} 1(\theta, j)), \mathrm{Y} 1(\theta, i))_{1,1}$

$+\lambda 1_{j}$ multiply $(\operatorname{transpose}(\psi 1(\theta, i)), \operatorname{Upsilonj} 1(\theta, j))_{1,1} d \theta / G$

\section{Para $\mathbf{m}=\mathbf{n}=\mathbf{2}$}

$>C 22:=(i, j)->(1 /(2 * G)) *$ int (

lambda2 [i]*multiply (transpose (psij2 (theta,j)),

(Upsilon2 (theta, $i))$ ) $[1,1]+$ lambda2 [j] * multiply (

transpose (psi2(theta, i) ), Upsilonj2(theta, j)) $[1,1]$, theta=alpha/2..alpha/2); 


$$
\begin{aligned}
C 22 & :=(i, j) \rightarrow \frac{1}{2} \int_{-1 / 2 \alpha}^{1 / 2 \alpha} \lambda 2_{i} \operatorname{multiply}(\operatorname{transpose}(\operatorname{psij} 2(\theta, j)), \mathrm{Y} 2(\theta, i))_{1,1} \\
& +\lambda 2_{j} \operatorname{multiply}(\operatorname{transpose}(\psi 2(\theta, i)), \operatorname{Upsilonj} 2(\theta, j))_{1,1} d \theta / G
\end{aligned}
$$

Definição dos valores $\alpha \mathbf{e} \lambda$

$\alpha$ (ângulo de abertura do material)

$>$ alpha : $=2 * \mathrm{Pi}$;

$$
\alpha:=2 \pi
$$

$\lambda$ positivo para o modo I (primeiro e segundo termos simétricos da expansão assintótica)

$>$ lambda1:=vector $([1 / 2,3 / 2])$;

$$
\lambda 1:=\left[\frac{1}{2}, \frac{3}{2}\right]
$$

$\lambda$ negativo para o modo I (primeiro e segundo termos simétricos da expansão assintótica)

$>\operatorname{lambdaj} 1:=\operatorname{vector}([-1 / 2,-3 / 2])$;

$$
\text { lambdaj } 1:=\left[\frac{-1}{2}, \frac{-3}{2}\right]
$$

$\lambda$ positivo para o modo II (primeiro e segundo termos anti-simétricos da expansão assintótica)

$>$ lambda2:=vector $([1 / 2,3 / 2])$;

$$
\lambda 2:=\left[\frac{1}{2}, \frac{3}{2}\right]
$$

$\lambda$ negativo para o modo II (primeiro e segundo termos anti-simétricos da expansão assintótica)

$>\operatorname{lambdaj} 2:=\operatorname{vector}([-1 / 2,-3 / 2])$;

$$
\text { lambdaj } 2:=\left[\frac{-1}{2}, \frac{-3}{2}\right]
$$

Cálculo dos coeficientes da função extratora

Valores da integral $C_{(i, j)}^{(m, n)}$ para m diferente de $\mathbf{n}$

$$
>\mathrm{C}(1,1) \text {; }
$$

$>\mathrm{C}(1,2)$;

$>\mathrm{C}(2,1)$;

$>\mathrm{C}(2,2)$; 
Valores da integral $C_{(\mathrm{i}, \mathrm{j})}^{(\mathrm{m}, \mathrm{n})}$ para $\mathbf{m}=\mathbf{n}=\mathbf{1}$

$>\operatorname{C11}(1,1)$;

$$
\frac{1}{2} \frac{-2 \pi-2 \kappa \pi}{G}
$$

$>\operatorname{C11}(1,2)$;

$$
0
$$

$>\operatorname{C11}(2,1)$;

0

$>\operatorname{C11}(2,2)$;

$$
\frac{1}{2} \frac{-6 \kappa \pi-6 \pi}{G}
$$

Valores da integral $C_{(i, j)}^{(m, n)}$ para $\mathbf{m}=\mathbf{n}=\mathbf{2}$

$>C 22(1,1)$;

$$
\frac{1}{2} \frac{-2 \pi-2 \kappa \pi}{G}
$$

$>\mathrm{C} 22(1,2)$;

$$
0
$$

$>\mathrm{C} 22(2,1)$;

$$
0
$$

$>C 22(2,2)$;

$$
\frac{1}{2} \frac{-6 \kappa \pi-6 \pi}{G}
$$


APÊNDICE C - Transformação de coordenadas para as derivadas da função extratora do método da função cutoff 
A expansão assintótica na vizinhança de uma região reentrante escrita na forma da equação (2.41) (Seção 2.3) fornece os campo de deslocamentos em coordenadas locais $\left(u_{\xi}, u_{\eta}\right)$ em função de coordenadas polares $(r, \theta)$ centradas na extremidade da região reentrante.

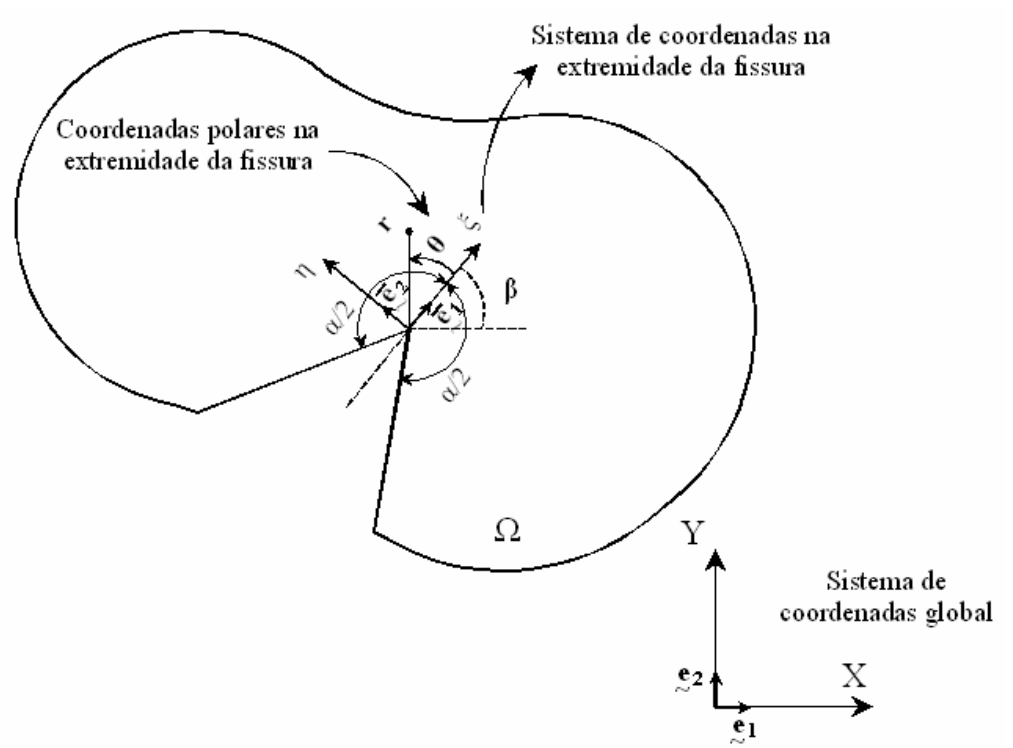

Figura C.1 - Sistemas de coordenadas local (extremidade da fissura) e global.

Na composição dos integrandos do MFC, faz-se necessário o cálculo das trações e das derivadas das tensões utilizando a função extratora.

Para o cálculo das trações nas arestas da região reentrante, desenvolve-se a transformação das derivadas de $\left(\mathrm{u}_{\xi}, \mathrm{u}_{\eta}\right)$ com relação às coordenadas polares na extremidade da fissura $(r, \theta)$ em derivadas de $\left(u_{x}, u_{y}\right)$ em relação às coordenadas globais (x,y) (Figura C.1).

Por questões de implementação computacional, as equações de equilíbrio em tensão, apresentadas nas integrais de área do MFC (Seção 3.3.2), foram utilizadas na forma de deslocamentos. As equações que formam o integrando da integral de área apresentada na formulação (3.38) podem ser substituídas da seguinte forma:

$$
\left\{\begin{array} { l } 
{ \frac { \partial \sigma _ { x } ^ { ( u ) } } { \partial x } + \frac { \partial \tau _ { x y } ^ { ( u ) } } { \partial y } } \\
{ \frac { \partial \tau _ { x y } ^ { ( u ) } } { \partial x } + \frac { \partial \sigma _ { y } ^ { ( u ) } } { \partial y } }
\end{array} \Rightarrow \left\{\begin{array}{l}
A \frac{\partial^{2} u_{x}}{\partial x^{2}}+B \frac{\partial^{2} u_{y}}{\partial x \partial y}+C \frac{\partial^{2} u_{x}}{\partial y^{2}} \\
A \frac{\partial^{2} u_{y}}{\partial y^{2}}+B \frac{\partial^{2} u_{x}}{\partial x \partial y}+C \frac{\partial^{2} u_{y}}{\partial x^{2}}
\end{array}\right.\right.
$$


para estado plano de tensão, tem-se:

$$
\mathrm{A}=\frac{\mathrm{E}}{\left(1-v^{2}\right)} ; \quad \mathrm{B}=\frac{\mathrm{E}}{2(1-v)} ; \quad \mathrm{C}=\mathrm{G}
$$

e para estado plano de deformação, tem-se:

$$
A=(2 G+\lambda) ; \quad B=(\lambda+G) ; \quad C=G
$$

E é o módulo de Young, $v$ é o coeficiente de Poisson, G (módulo de rigidez ao cisalhamento) e $\lambda$ estão relacionados com $E$ e $v$ na forma:

$$
\begin{aligned}
& G=\frac{E}{2(1+v)} ; \\
& \lambda=\frac{v E}{(1+v)(1-2 v)},
\end{aligned}
$$

A seguir, as transformações para as derivadas de primeira e de segunda ordens, para a função extratora do MFC, são descritas em notação indicial.

Considerando as definições apresentadas no apêndice A e definindo as seguintes representações para os sistemas de coordenadas e os campos de deslocamento:

- $\quad(\mathrm{r}, \theta) \rightarrow$ coordenadas polares do espaço loca;

- $\left(\overline{\mathrm{u}}_{\xi}(\mathrm{r}, \theta), \overline{\mathrm{u}}_{\eta}(\mathrm{r}, \theta)\right) \rightarrow$ campos de deslocamento no espaço local, em função das coordenadas polares locais;

- $\left(\tilde{\mathrm{u}}_{\xi}(\mathrm{x}, \mathrm{y}), \tilde{\mathrm{u}}_{\eta}(\mathrm{x}, \mathrm{y})\right) \rightarrow$ campos de deslocamento no espaço local, em função das coordenadas cartesianas globais;

Pretende-se calcular as derivadas de primeira e de segunda ordens do vetor,

$$
\{\overline{\mathrm{u}}(\mathrm{r}, \theta)\}=\overline{\mathrm{u}}_{\xi}(\mathrm{r}, \theta) \bar{\sim}_{1}+\overline{\mathrm{u}}_{\eta}(\mathrm{r}, \theta) \bar{\sim}_{2},
$$


e transformar em primeiras e segundas derivadas do vetor,

$$
\{\mathrm{u}(\mathrm{r}, \theta)\}=\mathrm{u}_{\mathrm{x}}(\mathrm{x}, \mathrm{y}) \underset{\sim}{\mathrm{e}}{ }_{1}+\mathrm{u}_{\mathrm{y}}(\mathrm{x}, \mathrm{y}) \underset{\sim}{\mathrm{e}} \text {. }
$$

Como os vetores bases $\underset{\sim}{\overline{\mathrm{e}}_{1}}$ e $\bar{\sim}_{2}$ (Figura C.1) são fixos, calculam-se apenas as transformações das derivadas dos componentes do vetor deslocamento. Esse cálculo pode ser feito para cada componente individualmente.

Sejam:

$$
\begin{aligned}
& \overline{\mathrm{g}}(\mathrm{r}, \theta)=\overline{\mathrm{u}}_{\xi}(\mathrm{r}, \theta) \text { ou } \overline{\mathrm{u}}_{\eta}(\mathrm{r}, \theta) \\
& \overline{\mathrm{x}}_{1}=\mathrm{r}, \quad \overline{\mathrm{x}}_{2}=\theta
\end{aligned}
$$

e sejam:

$$
\begin{aligned}
& \hat{g}(\xi, \eta)=\hat{u}_{\xi}(\xi, \eta) \text { ou } \hat{u}_{\eta}(\xi, \eta), \\
& \hat{x}_{1}=\xi, \hat{x}_{2}=\eta
\end{aligned}
$$

sendo assim, define-se:

$$
\hat{\mathrm{g}}(\xi, \eta)=\overline{\mathrm{g}} \circ \mathrm{T}_{1}^{-1}(\xi, \eta)
$$

sendo:

$$
\begin{gathered}
\mathrm{T}_{1}: \begin{array}{r}
\xi=\mathrm{r} \cos (\theta), \\
\eta=\operatorname{rsen}(\theta)
\end{array} \\
\mathrm{T}_{1}^{-1}: \mathrm{r}=\sqrt{\xi^{2}+\eta^{2}} \\
\theta=\arctan \left(\frac{\eta}{\xi}\right), \\
\mathrm{J}_{1}^{-1}(\mathrm{i}, \mathrm{k})=\frac{\partial \overline{\mathrm{x}}_{\mathrm{k}}}{\partial \hat{\mathrm{x}}_{\mathrm{i}}}=\left[\begin{array}{ll}
\frac{\partial \mathrm{r}}{\partial \xi} & \frac{\partial \theta}{\partial \xi} \\
\frac{\partial \mathrm{r}}{\partial \eta} & \frac{\partial \theta}{\partial \eta}
\end{array}\right]=\left[\begin{array}{ll}
\cos (\theta) & \frac{-\operatorname{sen}(\theta)}{\mathrm{r}} \\
\operatorname{sen}(\theta) & \frac{\cos (\theta)}{\mathrm{r}}
\end{array}\right],
\end{gathered}
$$


Sejam:

$$
\begin{aligned}
& \tilde{g}(\mathrm{x}, \mathrm{y})=\tilde{\mathrm{u}}_{\xi}(\mathrm{x}, \mathrm{y}) \text { ou } \tilde{\mathrm{u}}_{\mathrm{\eta}}(\mathrm{x}, \mathrm{y}), \\
& \mathrm{x}_{1}=\mathrm{x}, \quad \mathrm{x}_{2}=\mathrm{y}
\end{aligned}
$$

define-se:

$$
\tilde{g}(x, y)=\tilde{g} \circ T_{2}^{-1}(x, y)
$$

$\mathrm{T}_{2}$ é definida em (A.1) e $\mathrm{T}_{2}^{-1}$ pode ser escrita na forma:

$$
\begin{gathered}
\mathrm{T}_{2}^{-1}:\left\{\begin{array}{c}
\xi \\
\eta
\end{array}\right\}=[\mathrm{R}]^{\mathrm{T}}\left\{\begin{array}{l}
\mathrm{x}-\mathrm{x}_{\mathrm{o}} \\
\mathrm{y}-\mathrm{y}_{\mathrm{o}}
\end{array}\right\}, \\
{[\mathrm{R}]^{\mathrm{T}}=\mathrm{J}_{2}^{-1}(\mathrm{j}, \mathrm{m})=\frac{\partial \hat{\mathrm{x}}_{\mathrm{m}}}{\partial \mathrm{x}_{\mathrm{j}}}=\left[\begin{array}{ll}
\frac{\partial \xi}{\partial \mathrm{x}} & \frac{\partial \eta}{\partial \mathrm{x}} \\
\frac{\partial \xi}{\partial \mathrm{y}} & \frac{\partial \eta}{\partial \mathrm{y}}
\end{array}\right]=\left[\begin{array}{cc}
\cos (\beta) & -\operatorname{sen}(\beta) \\
\operatorname{sen}(\beta) & \cos (\beta)
\end{array}\right] .}
\end{gathered}
$$

Para as primeiras derivadas, tem-se:

$$
\begin{aligned}
& \hat{\mathrm{g}}_{, \mathrm{i}}=\frac{\partial \hat{\mathrm{g}}}{\partial \hat{\mathrm{x}}_{\mathrm{i}}}=\frac{\partial \overline{\mathrm{g}}}{\partial \overline{\mathrm{x}}_{\mathrm{k}}} \frac{\partial \overline{\mathrm{x}}_{\mathrm{k}}}{\partial \hat{\mathrm{x}}_{\mathrm{i}}}=\mathrm{J}_{1}^{-1}(\mathrm{i}, \mathrm{k}) \overline{\mathrm{g}}_{, \mathrm{k}} \\
& \tilde{\mathrm{g}}_{, \mathrm{j}}=\frac{\partial \tilde{\mathrm{g}}}{\partial \mathrm{x}_{\mathrm{j}}}=\frac{\partial \hat{\mathrm{g}}}{\partial \hat{\mathrm{x}}_{\mathrm{m}}} \frac{\partial \hat{\mathrm{x}}_{\mathrm{m}}}{\partial \mathrm{x}_{\mathrm{j}}}=\mathrm{J}_{2}^{-1}(\mathrm{j}, \mathrm{m}) \hat{\mathrm{g}}_{, \mathrm{m}}
\end{aligned}
$$

Portanto:

$$
\tilde{\mathrm{g}}_{, \mathrm{j}}=\mathrm{J}_{2}^{-1}(\mathrm{j}, \mathrm{m}) \mathrm{J}_{1}^{-1}(\mathrm{~m}, \mathrm{k}) \overline{\mathrm{g}}_{, \mathrm{k}}
$$

Agora:

$$
\{\mathrm{u}(\mathrm{x}, \mathrm{y})\}=\left\{\begin{array}{l}
\mathrm{u}_{\mathrm{x}} \\
\mathrm{u}_{\mathrm{y}}
\end{array}\right\}=[\mathrm{R}]\left\{\begin{array}{c}
\tilde{\mathrm{u}}_{\xi} \\
\tilde{\mathrm{u}}_{\mathrm{y}}
\end{array}\right\} .
$$

Como $[\mathrm{R}]$ é constante: 


$$
\mathrm{u}_{, \mathrm{j}}=\left\{\begin{array}{l}
\mathrm{u}_{\mathrm{x}, \mathrm{j}} \\
\mathrm{u}_{\mathrm{y}, \mathrm{j}}
\end{array}\right\}=[\mathrm{R}]\left\{\begin{array}{c}
\tilde{\mathrm{u}}_{\xi, \mathrm{j}} \\
\tilde{\mathrm{u}}_{\mathrm{\eta}, \mathrm{j}}
\end{array}\right\},
$$

$\tilde{\mathrm{u}}_{\xi, \mathrm{j}}$ e $\tilde{\mathrm{u}}_{\eta, \mathrm{j}}$ são calculados usando (C.18).

Para as derivadas de segunda ordem, tem-se:

$$
\begin{aligned}
& \frac{\partial^{2} \hat{\mathrm{g}}}{\partial \hat{\mathrm{x}}_{\mathrm{i}} \partial \hat{\mathrm{x}}_{\mathrm{j}}}=\hat{\mathrm{g}}_{\mathrm{ij}}=\frac{\partial}{\partial \hat{\mathrm{x}}_{\mathrm{i}}}\left(\frac{\partial \hat{\mathrm{g}}}{\partial \hat{\mathrm{x}}_{\mathrm{j}}}\right)=\frac{\partial}{\partial \hat{\mathrm{x}}_{\mathrm{i}}}\left(\frac{\partial \overline{\mathrm{g}}}{\partial \overline{\mathrm{x}}_{\mathrm{k}}} \frac{\partial \overline{\mathrm{x}}_{\mathrm{k}}}{\partial \hat{\mathrm{x}}_{\mathrm{j}}}\right) \\
& =\frac{\partial}{\partial \hat{\mathrm{x}}_{\mathrm{i}}}\left(\frac{\partial \overline{\mathrm{g}}^{\mathrm{g}}}{\partial \overline{\mathrm{x}}_{\mathrm{k}}}\right) \frac{\partial \overline{\mathrm{x}}_{\mathrm{k}}}{\partial \hat{\mathrm{x}}_{\mathrm{j}}}+\frac{\partial \overline{\mathrm{g}}^{2}}{\partial \overline{\mathrm{x}}_{\mathrm{k}}} \frac{\partial^{2} \overline{\mathrm{x}}_{\mathrm{k}}}{\partial \hat{\mathrm{x}}_{\mathrm{i}} \partial \hat{\mathrm{x}}_{\mathrm{j}}}, \quad, \\
& =\left(\frac{\partial^{2} \bar{g}}{\partial \bar{x}_{\mathrm{k}} \partial \bar{x}_{\mathrm{m}}} \frac{\partial \overline{\mathrm{x}}_{\mathrm{m}}}{\partial \hat{\mathrm{x}}_{\mathrm{i}}}\right) \frac{\partial \overline{\mathrm{x}}_{\mathrm{k}}}{\partial \hat{\mathrm{x}}_{\mathrm{j}}}+\frac{\partial \overline{\mathrm{g}}^{2}}{\partial \overline{\mathrm{x}}_{\mathrm{k}}} \frac{\partial^{2} \overline{\mathrm{x}}_{\mathrm{k}}}{\partial \hat{\mathrm{x}}_{\mathrm{i}} \partial \hat{\mathrm{x}}_{\mathrm{j}}}
\end{aligned}
$$

Sendo:

$$
\frac{\partial^{2} \overline{\mathbf{x}}_{\mathrm{k}}}{\partial \hat{\mathrm{x}}_{\mathrm{i}} \partial \hat{\mathbf{x}}_{\mathrm{j}}}=\mathrm{H}_{1}^{-1}(\mathrm{k}, \mathrm{i}, \mathrm{j}),
$$

Para $\mathrm{k}=1$ :

$$
\mathrm{H}_{1}^{-1}(1, \mathrm{i}, \mathrm{j})=\left[\begin{array}{cc}
\frac{\operatorname{sen}^{2}(\theta)}{\mathrm{r}} & -\frac{\operatorname{sen}(\theta) \cos (\theta)}{\mathrm{r}} \\
-\frac{\operatorname{sen}(\theta) \cos (\theta)}{\mathrm{r}} & \frac{\cos ^{2}(\theta)}{\mathrm{r}}
\end{array}\right]
$$

Para $\mathrm{k}=2$ :

$$
H_{1}^{-1}(2, i, j)=\left[\begin{array}{cc}
\frac{2 \operatorname{sen}(\theta) \cos (\theta)}{r^{2}} & \frac{2 \operatorname{sen}^{2}(\theta)-1}{r^{2}} \\
\frac{2 \operatorname{sen}^{2}(\theta)-1}{r^{2}} & \frac{2 \operatorname{sen}(\theta) \cos (\theta)}{r^{2}}
\end{array}\right]
$$

\section{Portanto:}




$$
\hat{\mathrm{g}}_{, \mathrm{ij}}=\mathrm{J}_{1}^{-1}(\mathrm{i}, \mathrm{m}) \overline{\mathrm{g}}_{, \mathrm{mk}} \mathrm{J}_{1}^{-1}(\mathrm{j}, \mathrm{k})+\overline{\mathrm{g}}_{\mathrm{k}} \mathrm{H}_{1}^{-1}(\mathrm{k}, \mathrm{i}, \mathrm{j})
$$

Continuando:

$$
\begin{aligned}
\frac{\partial^{2} \tilde{g}}{\partial x_{n} \partial x_{p}} & =\hat{g}_{, n p}=\frac{\partial}{\partial x_{n}}\left(\frac{\partial \tilde{g}}{\partial x_{p}}\right)=\frac{\partial}{\partial x_{n}}\left(\frac{\partial \hat{g}}{\partial \hat{x}_{q}} \frac{\partial \hat{x}_{q}}{\partial x_{p}}\right) \\
& =\frac{\partial}{\partial x_{n}}\left(\frac{\partial \hat{g}}{\partial \hat{x}_{q}}\right) \frac{\partial \hat{x}_{q}}{\partial x_{p}}+\frac{\partial \hat{g}}{\partial \hat{x}_{q}} \frac{\partial^{2} \hat{x}_{q}}{\partial x_{n} \partial x_{p}} \\
& =\left(\frac{\partial^{2} \hat{g}}{\partial \hat{x}_{q} \partial \hat{x}_{r}} \frac{\partial \hat{x}_{r}}{\partial x_{n}}\right) \frac{\partial \hat{x}_{q}}{\partial x_{p}}+\frac{\partial \hat{g}}{\partial \hat{x}_{q}} \frac{\partial^{2} \hat{x}_{q}}{\partial x_{n} \partial x_{p}}
\end{aligned}
$$

Sendo:

$$
\frac{\partial^{2} \hat{x}_{q}}{\partial x_{n} \partial x_{p}}=H_{2}^{-1}(q, n, p) .
$$

Portanto:

$$
\tilde{\mathrm{g}}_{\text {,np }}=\mathrm{J}_{2}^{-1}(\mathrm{n}, \mathrm{r}) \hat{\mathrm{g}}_{, \mathrm{rq}} \mathrm{J}_{2}^{-1}(\mathrm{p}, \mathrm{q})+\hat{\mathrm{g}}_{, \mathrm{q}} \mathrm{H}_{2}^{-1}(\mathrm{q}, \mathrm{n}, \mathrm{p}) \text {. }
$$

Utilizando-se as equações (C.25) e (C.28)é possível transformar as derivadas de $\overline{\mathrm{g}}$ em derivadas de $\tilde{\mathrm{g}}$. Neste caso, (C.27) é igual a zero.

Para as segundas derivadas dos campos de deslocamento, utilizando-se (C.19) e sendo $[\mathrm{R}]$ constante, tem-se:

$$
\mathrm{u}_{, \mathrm{ij}}=\left\{\begin{array}{c}
\mathrm{u}_{\mathrm{x}, \mathrm{ij}} \\
\mathrm{u}_{\mathrm{y}, \mathrm{ij}}
\end{array}\right\}=[\mathrm{R}]\left\{\begin{array}{c}
\tilde{\mathrm{u}}_{\xi, \mathrm{ij}} \\
\tilde{\mathrm{u}}_{\mathrm{\eta}, \mathrm{ij}}
\end{array}\right\},
$$

$\tilde{\mathrm{u}}_{\xi, \mathrm{ij}}$ e $\tilde{\mathrm{u}}_{\eta, \mathrm{ij}}$ são calculados usando (C.25) e (C.28).

Expandindo o desenvolvimento apresentado nesta seção, é possível encontrar uma relação matricial para as transformações das segundas derivadas. 